\title{
A numerical study of rotating interstellar clouds: equilibrium and collapse
}

Michael Lester Norman

(Ph.D. Thesis)

June 1980 
This report was prepared as an account of work sponsored by the United States Govemment. Neither the United States nor the United States Depariment of Energy, nor any of their employees, nor any of their contractors, subcontractors, or their employess, makes any warranty, express or implied, or assumes any legal liability or responsibility for the accuracy, sompleteness or usefulness of any information, apparatus, product of process disclosed, or represents that its use would not infringe privately owned rights.

Referance to a company or product name does not imply approval or recommendation of the product by the University of California or the U.S. Department of Energy to the exclusion of others that may be suitable.

Work performed under the auspices of the U.S. Department of Energy by the Lawrence Livermore Laboratory under Contract W-7405-Eng 48 . 


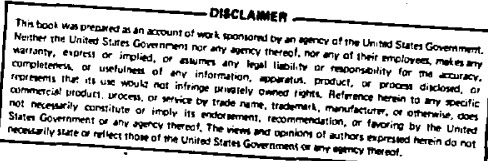

\title{
A numerical study of rotating interstellar clouds: equilibrium and collapse
}

\author{
Michael Leater Norman \\ (Ph.D. Thesis)
}

\section{Jone 1980}

A Dianertatien andmitued in partid atiafection of the requirements for the degree of

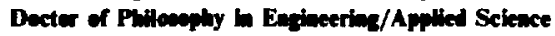

LAWRENCE LIVERMORE LABORATORY University of California • Livermore, California • 94550 


\section{ACKNOWLEDGEWENTS}

Jim wilson has been the guiding light in both the numerical and astrophysical aspects of this project. l gratefully thank him for letting me watch him work. Jim Leblanc and Paul Woodward have spent hours of their time sharing with me their special insights, and it is greatly appreniated. Special thanks also goes to William Newcanb, Peter Bodenheimer, David Black. Joel Tohline, Alan Boss, Frank Shu, and Larry Smarr tor useful discussions.

I can only inadequately thank Susan Tharp, my wife, for four years of patience, love, and support: Arthur and Yvonne Norman, my parents, for letting me roam; and Thomas Gill, who pointed me in the right direction. 
ABSTRACT

Equilibrium and collapse of rotating, axisymetric, idealized interstellar gas clouds is calculated with a $2 D$ hydrodynamics code. The hydrodynamics features an improved angular momentum advection algorithm. Angular momentum is advected consistently with mass by deriving angular momentum fluxes from mass fluxes and the local distribution of specific angular momentum. Local conservation is checked by a graph of mass versus specific angular momentum for the cloud as a whale.

The equilibrium structure of a rotating, isothermal cloud in contact with a constant intercloud pressure (the rotating analog of the Bonner-Ebert sphere) is computed for a variety of $\alpha=\left|E_{\text {thermal }} / E_{\text {gravitational }}\right|$, and $\beta=\mid E_{\text {rotational }}$ / Eravitational: for several distributions of angular momentum. Parameter space is searched by slowly cooling equilibrium clouds of fixed mass, angular momentum, and distribution of angular momentum in a fixed external pressure. Spheroidal and toroidal equilibria are found, depending on the distribution of angular momentum. Cooling sequences end in collapse. The critical line for collapse of spheroids with the angular momentum distribution of uniform, rigidly rotaling spheres of different angular momenta is tound empirically.

Isothermal collapse is calculated for a variety of initial and boundary conditions. Depending on the distribution of angular momentum, either a ring forma, or collapse continues to very high central densities preserving the disk topology of the cloud. In the latter case, the singular nature of the flow prohibits further integration. In the former case, a centrifugal rebound generates an off-axis density perturbation which grows to the point of 
gravitationally bindiag itself. A simple andysis is presented that shows how ring instability is related to the distribution of angular momentum.

Core formation during the nonisothermal phase of collapse is studied purely hydrodynamically (i.e., no radiation flow is included). The core evolves to a ring state when $\beta_{\text {core }} \geqslant 0.4$, which happens on a dynamic or accretion $t$ imescale depending on the angular momentum of the core. 
Chapter 1. Introduction 1

Chapter 2. Physical Model 5

$\begin{array}{ll}\text { 2.1 Equations } & \mathbf{5}\end{array}$

$\begin{array}{lr}2.2 \text { Symmetries } & 6\end{array}$

2.3 Boundary Conditions ?

2.4 Initial Conditions 9

Chapter 3. Numerical Model 10

$\begin{array}{lr}3.1 \text { Grid and Variables } & 10\end{array}$

$\begin{array}{ll}3.2 & \text { Difference Equations }\end{array}$

$\begin{array}{lr}3.3 \text { Stabiljty } & 41\end{array}$

$\begin{array}{lr}3.4 \text { Accuracy } & 42\end{array}$

Chapter 4. Rotating Isothermal Equilibrium 47

$\begin{array}{lr}\text { 4.1 Problem Statement } & 47\end{array}$

$\begin{array}{lr}4.2 \text { Procedure } & 48\end{array}$

4.3 Scaling and Parameterization 51

$\begin{array}{llr}4.4 \text { Results } & 52\end{array}$

$\begin{array}{lll}4.5 \text { Stability and Collapse B1 } & \text { Blitis }\end{array}$

Chapter 5. Rotating Isothermal Collapse 88

$\begin{array}{ll}5.1 \text { Introduction } & \text { 8B }\end{array}$

$\begin{array}{ll}5.2 \text { Collapse Iron Equjlibrium } & 90\end{array}$

5.3 Collapse from Rigidly Rotaling Spheres 98

5.4 Collapse from Differentially Rotating Spheres 108

An Archive of Graphical Output for Collapse Evolutions 113 


\section{Contents}

Chapter 6. Nonisothermal Evolution

6.1 Introduction

6.2 Parameters

6.4 Comments

Chapter 7. Discussion 199

7.1 A Simple Model of Rotating Isothermal Collapse 200

$\begin{array}{ll}7.2 \text { Ring Controversy } & 210\end{array}$

$\begin{array}{ll}7.3 \beta \text { and Ring Formation } & 211\end{array}$

7.4 Star Formation $\quad 212$

Chapter 8. Conclusions and Further Study 214

Appendix A. Test Problem:Rapidly Rotating Polytropes 216

Appendix B. Density and Angular Velocity at the Center 220

of a Collapsing Cloud

References 
CHAPTER I

\section{INTRODUCTION}

Star tormation remains an active field of astrophysical research. Star formation is studied theoretically, in part, by numerical simulations of gravitational collapse of interstellar gas clouds. The precise mechanisms which initiate collapse remain unclear. In most simulations a gravitationally unstable cloud is assumed from the outset.

The evolution of a parcel of interstellar material in a collapsing cloud is complicated by changes in thermodynamic state and by reaction to forces acting on it. Pressure, magnetic, rotational, gravitational, and perhaps even turbulent fores are in competition to determine th, trajectory of the parcel. The thermodynamic state of the parcel is determined by a competition between molecular dissocjation, atomic ionization, qrain evaporation, and the inverse processes. The thermodynamic state determines pressure and how magnetic fields and radiation couple to the parcel. A general collapse calculation would include all these eflects in a selt-consistent way with no symmetry restrictions on the solution (i.e., a 3D calculation). Such a calculation is not yet feasible and furthermore would be difficult to interpret. Research has proceeded by solving pieces of the puzzle starting with the simplest physical and symmetry assumptions. 
The study of equilibrium of nonrotating, spherically-symmetric gas clouds, and their collapse, including detailed thermodynamics and radiation hydrodynamics, is fairly well developed. Reviews by Woodward (1978), and Boder teimer and Black (1978) are recommended. For orientation purposes, the three major stages of spherical collapse are (Winkler and Newman 1980):

(1) isotherms.l free-fall collapse characterized by an ever-increasing density contrast from cloud center to edge

(2) formation of a hydrostatic core due to opacity effects, which acts as an accretion center for the infalling envelope

(3) slow, quasistatic evolution of the core as it accretes the remainder of the envelope

Research on cloud collapse in two or three dimensions has scarcely solved the isothermal collapse phase, due primarily to the numerical difficulties of computing the highly convergent flow. This research studies the equilibrium and collapse of rotating, axisymmetric interstellar clouds with a two-dimensional hydrodynamics code. We pose the following questions:

(1) What are the equilibrium states for a rotating, isothermal cloud? (the rotating analogs of Bonner-Ebert spheres)

(2) Wha: are the generic features of isothermal collapse?

(3) Which states does collapse connect?

(4) What are the significant dynamic effects in the nonisothermal phase of the evolution?

The equilibrium problem (1) has not previously been studicd to the best of the author's knowledge. Previous work on rotating collapse remains controversial, however. Some authors (Larson 1972; Black and Bodenheimer 1976; Nakazawa, Hayashi, and Takahara 1976; Boss 1979) calculated that collapse 
produces a gravitationally bound ring which grows from a centrifugally generated off-axis density perturbation. Other authors (Tscharnuter 1975; Fricke, Mollenhoff, and Tscharnuter 1976; Kamiya 1977), using the same initial and boundary conditions but sigaficantly different numerical techniques, found a flattened disk-Jike configuration. The evolutions become discrepant in the isothermal phase of collapse. Norman, Wilson, and Barton (1980) showed that a ring can be produced by numerical diffusion of angular momentum. When this source of error was reduced, they found a runaway collapse ax.. flattening of the cloud leading to what appeared to be a singular isothermal disk.

The interest in $r$ ings stems from the possibility that they play an important role in cloud fragmentation. Norman and Wilson (1978) calculated the 3D evotution oi an isothermal $r$ ing and found it fragmented rapidly into two or three bodies. Furthermore, they fuund the spin angusar momentum per unit mass of the fragments to be roughly an order of magnitude less than the parent ring/cloud. Bodenheimer (1978) constructed a hierarchical cloud fragmentation scenario based on ring formaticn and fragmentation, and was able to reproduce observed binary star characteristics. Thus, the "angular momentum problem" (Bodenheimer and Black 1978) appears to be solvable by the conversion of spin to orbital angular momentum through ring fragmentation. One particular aim of this work is therefore finding under which conditions equilibrium or collapse problems have ring solutions. It is found that the distribution of angular momentum in the cloud has an important effect in this regard.

The physical and numerical models are described in chapters 2 and 3 , respectively. Rotating isothermal equilibria are generated with the hydrodynamic code a. I require a dynamic cloud boundary for the application of a pressure boundary condition. The multimaterial method for acsomplishing this is 
also described. Question 1 is addressed in chapter 4, where rotating, isothermal equilibria (the rotating analogs of Bonner-Ebert spheres) are presented. Questions 2 and 3 ere considered in chapter 5, where a number of isothermal collapse calculations are described. Question 4 is addressed in chapter 6. where core formalion in the nonisothermal phase of evolution is described. The results of chapters 4 and 5 regarding structure and evolution of rotating isothermal clouds are discussed in chapter 7 . A simple analysis is presented that shows the effect of angular momentum distribution on ring formation in pulely isothermal collapse. Conclusions and recommendations for further study are presented in chapter. 8. 
CHAPTER 2

PHYSICAL MODEL

\section{1) Equations}

The cloud is assumed to obey the equations of ideal fluid flow, which in Eulerian form comprises

$$
\begin{aligned}
& \frac{\partial \rho}{\partial \mathrm{t}}+\nabla \cdot \rho \underline{v}=0 . \\
& \frac{\partial \rho \underline{v}}{\partial \mathrm{t}}+\nabla \cdot \rho \underline{\underline{\nu}}+\rho \nabla \Phi+\nabla \mathrm{P}=0, \\
& \frac{\partial \mathrm{E}}{\frac{\partial \mathrm{t}}{\partial}}+\nabla \cdot \mathrm{E} \underline{\underline{v}}+\mathrm{P} \underline{\nabla} \cdot \underline{\underline{v}}=0 .
\end{aligned}
$$

Here $p$ is the mass density. $v$ the fluid velocity, $\$$ the gravitational potential. and $E$ the internal energy density $\left(\mathrm{erg} \mathrm{cm}^{-3}\right)$. $E$ is related to the pressure, $P$, by the ideal gas law

$$
P=(\gamma-1) E \text {. }
$$

The gas is assumed to be molecular hdrogen. The adiabatic index, $\gamma$, is taken as simply $7 / 5$. The gravitational potential is related to the mass density through the Poisson equation,

$$
\nabla^{2}=4 \pi \mathrm{G} \rho
$$

and closes the system of equations.

For the isothermal calculations, the energy density is computed by simply 
multiplying the mass denaity by a constant apecific internal energy $c$, thus $E=$ or. The energy equation (3) is not solved in this case.

When simulating the nonisothermal phase of protostellar evolution (due to trapping of radiation), material is put on an adiabat of index $\gamma$ (or densities greater than a critical density $\rho_{0}$ :

$$
P=\left\{\begin{array}{ll}
(\gamma-1) \rho \varepsilon & \rho<\rho_{0} \\
(\gamma-1) \rho \varepsilon\left(\rho / \rho_{0}\right)^{\gamma-1} & \rho>\rho_{0}
\end{array} .\right.
$$

A better treatment of the nonisothermal phase of protostellar evolution would include radiative transport in the opaque regions, and a detailed model of the thermodynamics of a gas-grain mixture (Black and Bodenheimer 1975). Such a treatment is an extensive additional computational eflort in two or more dimensions and not approprince at this early stage of research. We are interested only in the formation of a hydrostatic core in rotating collapse, and watching its oscillations as equilibrium is approached. It is hoped that this idealized treatment yields results qualitatively the same as would the full-blown treatment.

\section{2) Symmetries}

Equations $(1-5)$ are solved in cylindrical coordinates $(R, 6, Z)$. The rotation axis of the cloud coincides with the 1 ine $R=0$. Axisymetry and equatorial symmetry are assumed in the flow varjables, so terms involving $\partial / \partial \theta$ do not appear. The assumption of equatorial symmetry simplifies the solution of the Poisson equation (see sec, 2.3 ).

Writing equations $(1-3,5)$ in the torm they are differenced, we have 


$$
\begin{aligned}
& \frac{\partial \rho}{\partial \mathrm{t}}=-\frac{\partial \rho \mathrm{U}}{\partial \mathrm{Z}}-\frac{\mathrm{I} \partial \mathrm{R} \rho \mathrm{V}}{\mathrm{R}} \\
& \text { OS OSU IORSV aP } \partial \Phi \\
& \overline{\partial t}=-\frac{-}{\partial Z}-\frac{-}{\mathrm{R} \partial \mathrm{R}}-\overline{\partial Z}-\rho-\text {. } \\
& \frac{\partial T}{\partial t}=-\frac{\partial T U}{\partial Z}-\frac{1 \partial R T V}{R \partial R}-\frac{\partial P}{\partial R}-\frac{\partial \Phi}{\partial R}+\rho \Omega^{2} R \text {, } \\
& \text { OA DAU IJRAV } \\
& \overline{\partial t}=-\frac{-}{\partial Z}-\bar{R} \frac{}{\mathrm{R} R} . \\
& \text { aE aEU } 1 \text { aREV aU } 10 \mathrm{RV}
\end{aligned}
$$

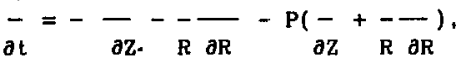

$$
\begin{aligned}
& \partial^{2} \phi \quad 1 \partial \quad \partial \phi \\
& -+-R-=4 \pi G \rho \text {. } \\
& \partial Z^{2} \text { RaR } \partial R
\end{aligned}
$$

Here the components of momentum in the $Z$ and $R$ directions are $S$ and $T$ respectively. The angular momentum density is $A=\rho \cap R^{2}$; $\Omega$ being the local angular velocity. The velocity components are defined as $U=S / \rho, V=T / \rho, W=$ A/ $\rho R$. The momentum densities are carried as primary variables rather than velocities because the divergence terms lend to straight-forward, conservative numerical approximations.

\section{3) Boundary Conditions}

Hydrodynamic and gravitational potential boundary values are required to complete the problem. Since our domain of interest is one quadrant in the meridional plane, boundary values along the equator and rotation axis are given by synatry. We have

$$
\begin{array}{ll}
-(R, Z=0)=0, & \frac{\partial \phi}{\partial R}(R=0, Z)=0 . \\
U(R, Z=0)=0 . & V(R=0, Z)=0 .
\end{array}
$$


The boundary values of gravitational potential on some exterior curve $B$ are computer from a multipole expansion.

$$
\Phi_{B}=-\mathrm{G} \sum_{l=0}^{q} \mathrm{P}_{l}\left(\mu_{B}\right) r_{D}-(l+1) m_{l} .
$$

and the multipole moments are given by

$$
m_{l}=\int d^{3} r \rho(r) r^{l} P_{l}(\mu) \text {. }
$$

Here $r$ is the position vector, $r$ is its magnitude, $\mu$ is the cosine of the angle between the rotation axis and the vector $r$, and $P_{\ell}$ are the Legendre polynomials. With the assumed equatorial symmetry odd moments vanish. In practice only the $l$ $=0,2$, and 4 terms are used. This has proven sufficient provided the boundary $B$ is somewhat removed from the cloud boundary. In practice $B$ is taken as the edge of the computational grid.

In light of our ignorance of the physical conditions in an interstellar cloud just prior to gravitational collapse, the hydrodynamic boundary conditions are somewhat free to choice. Two kinds of boundary conditions are used in this research. The curve $B^{\prime}$ on which they are defined need not coincide with the boundary curve $B$ used for the potential. The first is called constant volume in which the components of velocity normal to $B^{\circ}$ are set to zero. We take $B^{\circ}$ to be a spherical surface of radius equal to the initial radius of a spherical cloud. Used for some of the collapse problems, the cloud evolves inside a spherical box. Although this boundary condition is somewhat unphysical, it has been used in previous research because it is easy to implement in an Eulerian code. The second boundary condition used is the application of a constant pressure on curve $B^{\prime}$ where $B^{\prime}$ is the surface of the cloud. Since $B^{\prime}$ is a free boundary, a multimaterial capability is required to implement this boundary condition in an Eulerian code. The pressure boundary condition simulates the contact of a cool 
interstellar cloud with hot intercloud medium. Work with less realistic pressure boundary conditions have shom them to be of limited value.

\section{4) intial Conditions}

$\because$ basic initial condition for both equilibrium and collapse problems is a uniform ciensity sphere in rotational motion only. The angular momentum and rotation law are varied to generate a family of initial conditions. The angular velocity profile is limited to a linear function of distance from the rotation axis, parameterized by the ratio of edge to central angular velocities. s. The sphere is evolved nydrodynamically and either finds an equilibrium state or collapses, depending on the specific internal energy of the gas. $\varepsilon$. Since isothermal problems scale, the value of $\varepsilon$ is only meaningful in relation to the other cloud parameters: mass, $\mathcal{M}$, radius, $R_{0}$, angular momentum, $J$, and surface pressure, $P_{x}$. Here we adopt the scale of a one solar mass cloud with $R_{0} \sim 10^{17}$ $\mathrm{cm}$, and $\varepsilon \sim 10^{\circ} \mathrm{erg} \mathrm{gm}^{-1}(\mathrm{~T} \sim 10 \mathrm{~K})$. Parameters and scaling laws are given for the numerical examples described in chapters 4-6. 
CHAPTER 3

\section{NUMERICAL MODEL}

Equations (7-12) of chapter 2 are solved via finite difference methods cast on a cylindrical computational mesh $(R, Z)$. Typically $48^{2}$ zones are used to represent one quadrant of the cloud. The major fealures of this method are:

(1) a moveable grid that collapses with the cloud to maintain resolution

(2) a superior angular momentum advection algorithm that preserves the specific angular monentum of advected mass.

(3) a dynamic cloud boundary on which a pressure boundary condition is applied

The grid, difference equations, slability, and accuracy of the method are now described in more detail.

3.1) Grid and Variables

The computational grid is shown in figure 3.1. A zone is defined by the four corners $(k, j),(k+1, j),(k, j+1)$, and $(k+1, j+1)$. Other zone measurements used in forming the difference equations are shown in figure 3.2. In general the zone spacing is not uniform. We have the following definitions:

$$
\begin{aligned}
& R_{j+1 / 2}=\left(R_{j}+R_{j+l}\right) / 2 \\
& \delta R_{j+1 / 2}=\left(R_{j+1}-R_{j}\right)
\end{aligned}
$$




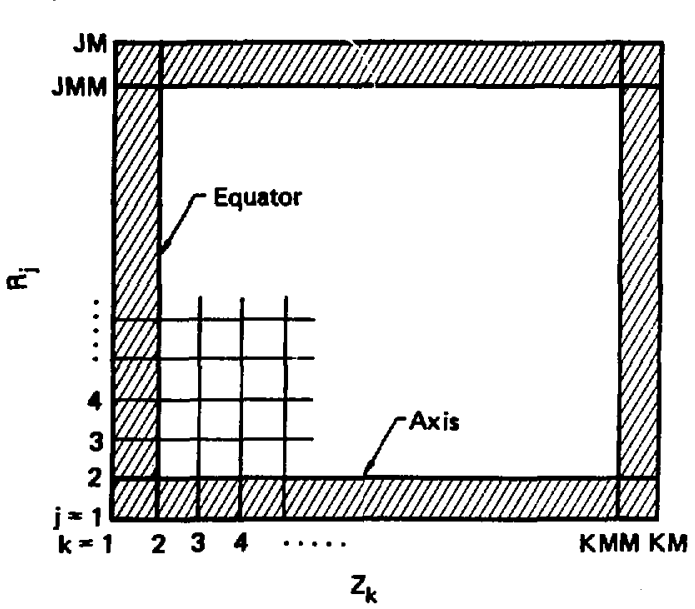

Figure 3.1--The computational grid. A zone is defined by the four corners $(k, j),(k+1, j),(k, j+1)$, and $(k+1, j+1)$. The dynamic zones lie inside the boundary zones (hatched region). The equator and rotation axis are lines $Z_{2}$ and $R_{2}$, respectively. 


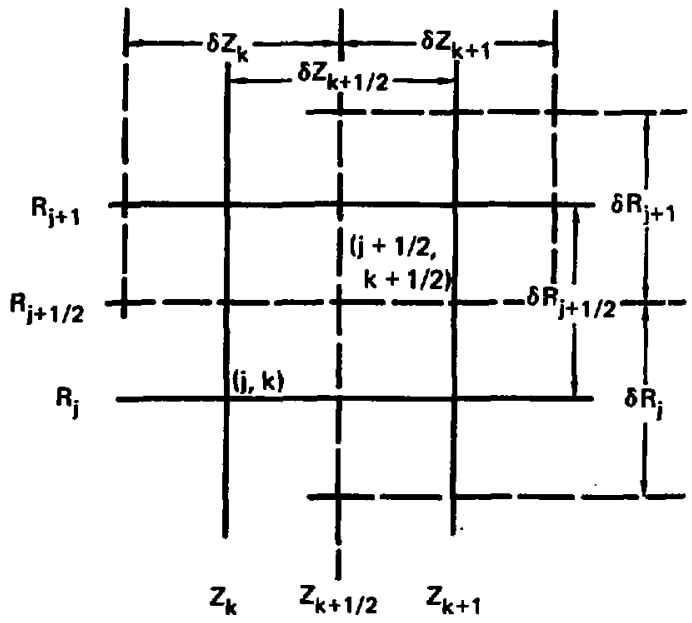

Figure 3.2--Zone measurements for the staggered grid. The point $\left(R_{j+1 / 2}, Z_{k+1 / 2}\right)$ lies at the center of zone $(j, k)$. The zone spacing is not uniform in general. 


$$
\delta R_{j}=\left(R_{j+1 / 2}-R_{j-1 / 2}\right),
$$

and analogously for $\mathrm{Z}_{k+1 / 2}, \delta \mathrm{Z}_{\mathrm{k}+1 / 2}, \delta \mathrm{Z}_{\mathrm{k}}$. The volume of the zone centered at $(k+1 / 2, j+1 / 2)$ is simply

$$
V o I_{k+1 / 2 j+1 / 2}=2 \pi R_{j+1 / 2} \delta R_{j+1 / 2} \delta Z_{k+1 / 2}
$$

The coordinate lines are allowed to move as the cloud collapses, but remain orthogonal. A variety of methods are used for grid motion, as described in section 3.2.5. However the essential feature is that the grid speeds are calculated indirectly by first moving the mesh so as to keep resolution and zoning regularily, and then deriving the grid speeds through the first-order prescription

$$
\begin{aligned}
& U_{g, k}^{n}=\left(Z_{k}^{n}-Z_{k}^{n-1}\right) / \delta L^{n-1} \\
& V_{g, j}^{n}=\left(R_{j}^{n}-R_{j}^{n-1}\right) / \delta t^{n-1}
\end{aligned}
$$

Here $n$ is the index for the $t$ imelevel, and $\delta t^{n}=t^{n}-t^{n-1}$.

The loeations of the dynamic variables $\rho, E, \phi, S, T, A$ in a zone are shown in figure 3.3. Scalar quantities $\rho, E, \Phi$ are defined at the zone center while the components of I inear momentum are face centered. This staggered arrangement makes for simple difference equations for advection and acceleration (except centrifugal acceleration). The angular momentum density is localed with the mass density so that zonal specific angular momenta are simply defined, i.e.. require ro averaging as in the scheme used by Black and Bodenheimer (1975). The centrifugal acceleration requires averaging two zones, however.

From these primary variables we derive velocity components $U$, $V$ whenever needed, which are given by

$$
u_{k j+1 / 2}=\frac{S_{k j+1 / 2}}{\bar{\rho}_{k j+1 / 2}} \text {, }
$$




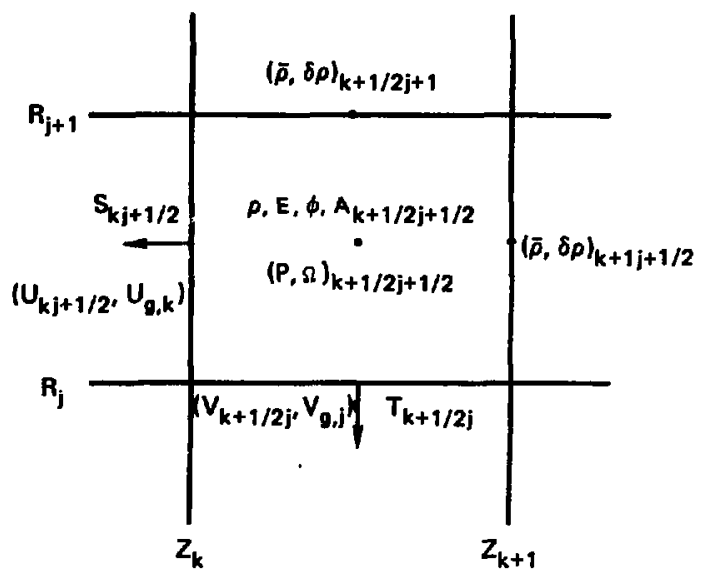

Figure 3.3--The centering of variables within a zone. Primary variables $\rho$. $E$, $\Phi$, and $A$ are zone centered; primary variables $S$ and $T$ are $f$ ace centered. Derived quantities are shown in parenthesis, as defined in text. 


$$
v_{k+1 / 2 j}=\frac{T_{k+1 / 2 j}}{\bar{\rho}_{k+1 / 2 j}}
$$

Here we have defined face-centered average densities

$$
\begin{aligned}
\bar{\rho}_{k j+1 / 2} & =\frac{\left(\rho_{k+1 / 2 j+1 / 2}+\rho_{k-1 / 2 j+1 / 2}\right)}{2} . \\
\bar{\rho}_{k+1 / 2 j} & =\frac{\left(\rho_{k+1 / 2 j+1 / 2}+\rho_{k+1 / 2 j-1 / 2}\right)}{2} .
\end{aligned}
$$

We also define face-centered density differences to be used later on:

$$
\begin{aligned}
\delta \rho_{k j+1 / 2}= & \frac{\left(\rho_{k+1 / 2 j+1 / 2}-\rho_{k-1 / 2 j+1 / 2}\right)}{2} . \\
\delta \rho_{k+1 / 2 j}= & \frac{\left(\rho_{k+1 / 2 j+1 / 2}-\rho_{k+1 / 2 j-1 / 2}\right)}{2} .
\end{aligned}
$$

The angular velocity of a zone, $\Omega$, is defined by assuming rigid rotation inside the zone:

$$
\Omega_{k+1 / 2 j+1 / 2}=\frac{2 A_{k+1 / 2 j+1 / 2}}{\rho_{k+1 / 2 j+1 / 2}\left(R_{j+1}^{2}+R_{j}^{2}\right)} .
$$

The pressure is derived from $\rho$ and $\varepsilon$ through the equation of state. The derived quantities are shown in parenthesis in figure 3.3 .

\section{2) Difference Equations}

The hydrodynamic difference equations are explicit (i.e forward time differenced), operator-spitit, first-order accurate in time, and second-order accurate in space. All variables ere advanced from time level $n$ to $n+1$ by a succession of numerical operations representing the terms on the right-hand-sides of equations $(7-11)$ of chapter 2 . The Poisson equation is differenced to second-order spatial accuracy, and solved iteratively using, an 
Ad) l method described by Black and Bodenheimer (1975). The difference equationa for thesc operations are described in the order as implemented in an actual problem cycle. The steps are summarized in $f$ igure 3.4 .

\subsection{1) Acceleration}

The acceleration due to pressure, gravity, and centrilugal lorces is given in component form as

$$
\begin{gathered}
\frac{\left(S^{a}-S\right)_{k j+1 / 2}}{\delta t}=-\frac{\left(P_{k+1 / 2 j+1 / 2}-P_{k-1 / 2 j+1 / 2}\right)}{\delta Z_{k}} \\
-\bar{\rho}_{k j+1 / 2} \frac{\left(\Phi_{k+1 / 2 j+1 / 2}-\Phi_{k-1 / 2 j+1 / 2}\right)}{\delta Z_{k}} .
\end{gathered}
$$

and

$$
\begin{aligned}
& \frac{\left(T^{n}-T\right)_{k+1 / 2 j}}{\delta t}=-\frac{\left(P_{k+1 / 2 j+1 / 2}-P_{k+1 / 2 j-1 / 2}\right)}{\delta R_{j}} \\
& -\bar{\rho}_{k+1 / 2 j} \frac{\left(\Phi_{k+1 / 2 j+1 / 2^{-\Phi} k+1 / 2 j-1 / 2}\right)}{\delta R_{j}} \\
& +\bar{p}_{k+1 / 2 j} \frac{\left(\Omega_{k+1 / 2 j+1 / 2}+\Omega_{k+1 / 2 j-1 / 2}\right)^{2} R_{j}}{4} .
\end{aligned}
$$

Variables without superscripts are at timelevel n. All spatial differences and averuges are centered with the momentum components, so second-order spatial accuracy is obtained.

\subsection{2) Artificial Viscosity}

An artificial viscosity patterned after von Neumann's treatment is used to handle flow discontinuities arising in shocks. Compressional velocity gradients 


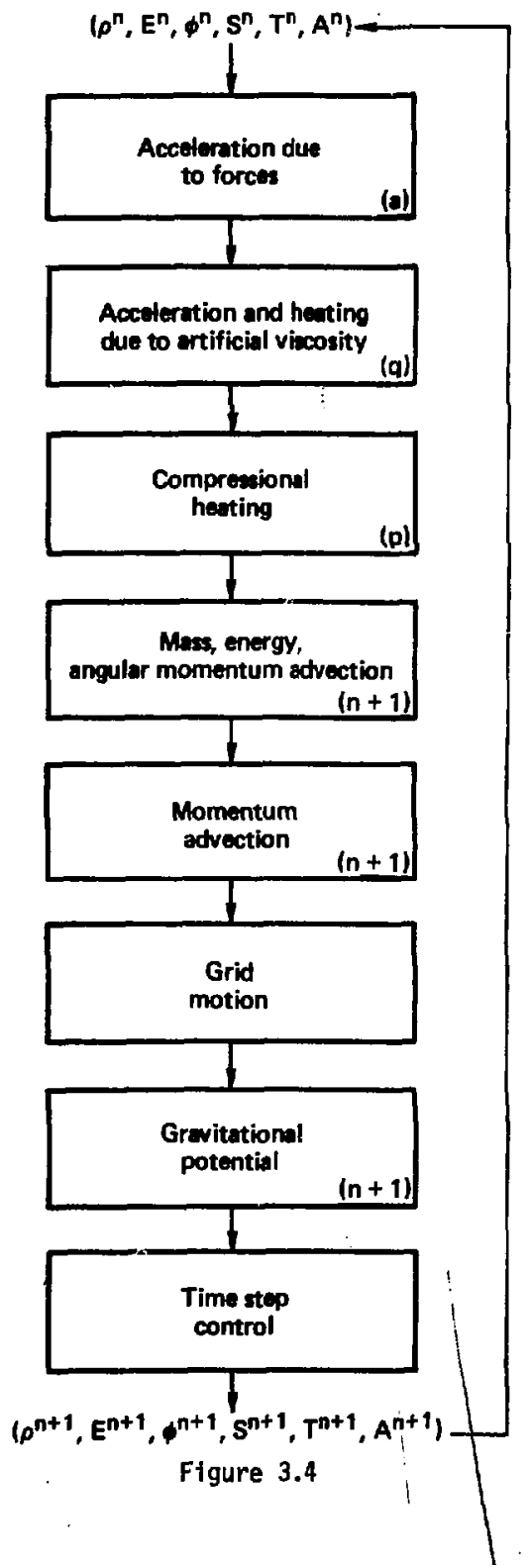


Figure 3.4--The hydrodynamic cycle. Variables are advanced from timelevel $n$ to $n+1$ by a series of intermediate operations shown in boxes. The characters in the lower-right corners appear in the lext as superscripts for the intermediate steps. 
are used like pressures to accelerate and heat the material. Define stress components

$$
\begin{aligned}
& Q_{k+1 / 2 j+1 / 2}^{Z Z}= \begin{cases}q \rho_{k+1 / 2 j+1 / 2}\left(U_{k+1 j+1 / 2}^{\ell}-U_{k j+1 / 2}^{k}\right)^{2} & \text { (Compression) } \\
0 & \text { (Expansion) }\end{cases} \\
& Q_{k+1 / 2 j+1 / 2}^{R R}=\left\{\begin{array}{ll}
q \rho_{k+1 / 2 j+1 / 2}\left(V_{k+1 / 2 j+1}^{a}-V_{k+1 / 2 j}^{a}\right)^{2} & \text { (Compression) } \\
0 & \text { (Expansion) }
\end{array}\right. \text {, }
\end{aligned}
$$

where the constant. $q$, is typically 2 , and $U^{n}$, $V^{a}$ are calculated from $S^{a}, T^{a}$ through equations $(4,5)$. The momentum components are "Q" accelerated according to

$$
\frac{\left(S^{q}-S^{D}\right)_{k j+1 / 2}}{\delta t}=-\frac{\left(Q_{k+1 / 2 j+1 / 2^{-Q}}^{Z Z} Z \mathbf{Z}-1 / 2 j+1 / 2\right)}{\delta Z_{k}} .
$$

and

$$
\frac{\left(T^{q}-T^{a}\right)_{k+1 / 2 j}}{\delta t}=-\frac{\left(Q^{R R} k+1 / 2 j+1 / 2^{-Q^{R R}} k+1 / 2 j-1 / 2\right)}{\delta R_{j}} .
$$

Loss of kinetic energy is balanced by an artificial viscous heating term in the energy equation:

$$
\begin{aligned}
& \frac{\left(E^{q}-E\right)_{k+1 / 2 j+1 / 2}}{\delta t}=-Q_{k+1 / 2 j+1 / 2}^{Z Z} \frac{\left(U_{k+1 j+1 / 2}^{Q}-U_{k j+1 / 2}^{Q}\right)}{\delta Z_{k}} \\
& -Q_{k+1 / 2 j+1 / 2}^{R R} \frac{\left(V_{k+1 / 2 j+1}^{a}-V_{k+1 / 2 j}^{a}\right)}{\delta R_{j}} .
\end{aligned}
$$

\subsection{3) Compressional Heating}

In the absense of heat sources and sinks, the compressional heating is given by the differential form of the first law of thermodynamics. 


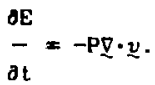

For this equation an effort is made to bcheive second-order accuracy in time because this has been found to substantially improve energy conservation. Let $E^{p}$ be the internal energy density after compressional beating. We form a time-centered pressure by averaging $p^{n}$ and $(\gamma-1) E^{p}$. We then write

$$
\frac{\left(E^{P}-E^{Q}\right)_{k+1 / 2 j+1 / 2}}{\delta t}=-\frac{\left[P+(\gamma-1) E^{P}\right]}{2} \nabla \cdot \underline{y}_{k+1 / 2 j+1 / 2}
$$

where here

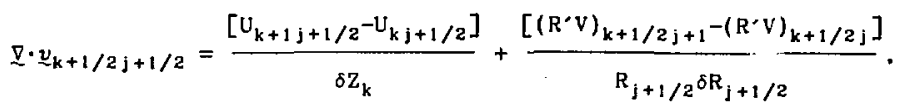

and

$$
R_{k+1 / 2 j}^{\prime}=R_{j}+\delta t V_{k+1 / 2 j} / 2 \text {. }
$$

The velocity components, $U$ and $V$, are derived $f$ rom $S^{q}$ and $T^{q}$ by equations $(4,5)$, and will be used without superscripts in the sequel. Equation (15) can be manipulated into the simple algebraic form

$$
E_{k+1 / 2 j+1 / 2}=\frac{\left[E^{9}-P \frac{\delta t \underline{\nabla} \cdot \underline{v}}{2}\right]_{k+1 / 2 j+1 / 2}}{\left[1+(\gamma-1) \frac{\delta t \underline{\nabla} \cdot \underline{v}}{2}\right]_{k+1 / 2 j+1 / 2}} .
$$

This procedure explicitly assumes the gamma-law equation of state for the time-centered pressure in equation (15). Experience has shown that energy conservation is improved by using the same pressure in equation (18) as is used for acceleration, rather than, say, $(\gamma-1) \mathrm{E}^{q}$.

For the isothermal calculations a constant temperature is maintained by edict, rather than by adding radiative loss terms to the energy equation. 
Therefore $E$ is computed by simply multiplying the mass density by a specific internal energy which is input to the calculation. This slep is actually done after new densities are found by the advection calculation, described next.

\subsection{4) Advection}

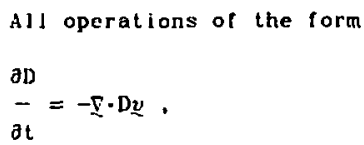

where $D$ is $o, E, S$, T, and $A$ are treated in this section. Physically, this equation describes a cortinuous remapping of Lagrangian fluid elements inta a fixed frame of reference (the Eulerian frame.) Written in integral form, we have

$$
\frac{\partial}{\partial t} \int_{d^{3} x D} D=-\oint_{S} \mathrm{D} \underline{v} \cdot \mathrm{dg} .
$$

Equation ( 19 ) describes changes in $D$ due to flow in and out of a region or zone bounded by surface 2 . When fluid flows from one zone to another in a numerical calculation. numerical diffusion results. The amount of diffusion depends on how accurately equation (19) is approximated, and how well zoned the calculation is. The approximalions deseribed below are second-order accurate in space and first-order accurate in time. An imporlant leature of the advection scheme is that internal energy, momentum, and angular momentum are advected in a manner consistent with mass advection. That is, rather than moving all quantities independently by some difference approximation to (19), mass is moved, and with it is carried the internal energy, linear momentum, and angular momertum appropriate to it. This is achjeved by using information on the distribution of the specific internal energy, the velocity, and the specific angular momentum inside zones. 


\section{Mass}

Mass is advected by differencing the integral form of the continuily equation,

$$
\boldsymbol{u}^{\mathrm{n}+1}=\boldsymbol{u}^{\mathrm{n}}-\int_{\Omega}^{\mathrm{dt} \oint_{\rho} \underline{\mathrm{d}} \cdot \mathrm{d} \underline{a}},
$$

where $\mu$ is the mass bounded by a closed surface $s$, and the mass clux oy is taken relative to the area element $\mathrm{d} g$, which can be moving. Applying this to the zone shown in Figure 3.5 , we write

$$
\begin{aligned}
& \mu_{k+1}^{n+1} / 2 j+1 / 2 \\
& +M_{k+1 / 2 j+1 / 2}^{n} \\
& +\left(\delta \mu_{k+1 / 2 j+1}-\delta M_{k+1 / 2 j}+\delta \mu_{k+1 j+1 / 2}-\delta M_{k j+1 / 2}\right),
\end{aligned}
$$

where the mass fluxes are defined

$$
\begin{aligned}
& \delta \ell_{\mathrm{k}+1 / 2 \mathrm{j}}=\Delta_{\mathrm{k}+1 / 2 \mathrm{j}} \tilde{\rho}_{\mathrm{k}+1 / 2 \mathrm{j}} A_{\mathrm{k}+1}^{\mathrm{n}+1 / 2} / 2, \\
& s \ell_{\mathrm{kj}+1 / 2}=\Delta_{\mathrm{kj}+1 / 2} \tilde{\rho}_{\mathrm{kj}+1 / 2^{A} \mathrm{j}+1 / 2},
\end{aligned}
$$

with

$$
\begin{aligned}
& \Delta_{k+i / 2 j}=-\left(v_{k+1 / 2 j}-v_{g, j}\right) \delta t . \\
& \Delta_{k j+1 / 2}=-\left(U_{k j+l / 2}-U_{g, k}\right) \delta t .
\end{aligned}
$$

The velocity components have been calculated from the most recent momentum components, $\mathrm{S}^{\mathrm{q}}$ and $\mathrm{T}^{\mathrm{q}}$, by equations $(4,5)$. We have defined advection densities, $\not$. where refering to equations $(6,7)$,

$$
\begin{aligned}
& \tilde{\rho}_{k+1 / 2 j}=\bar{\rho}_{k+1 / 2 j}+\frac{\Delta_{k+1 / 2 j}}{\delta R_{j}} \delta \rho_{k+1 / 2 j} . \\
& \tilde{\rho}_{k j+1 / 2}=\bar{\rho}_{k j+1 / 2}+\frac{\Delta_{k j+1 / 2}}{\delta Z_{k}} \delta \rho_{k j+1 / 2} .
\end{aligned}
$$

The areas of the zone faces $\left(A_{k j+1 / 2}, A_{k+1 / 2 j}\right)$ are $t$ ime centered to correct for grid motion to second-order accuracy: 


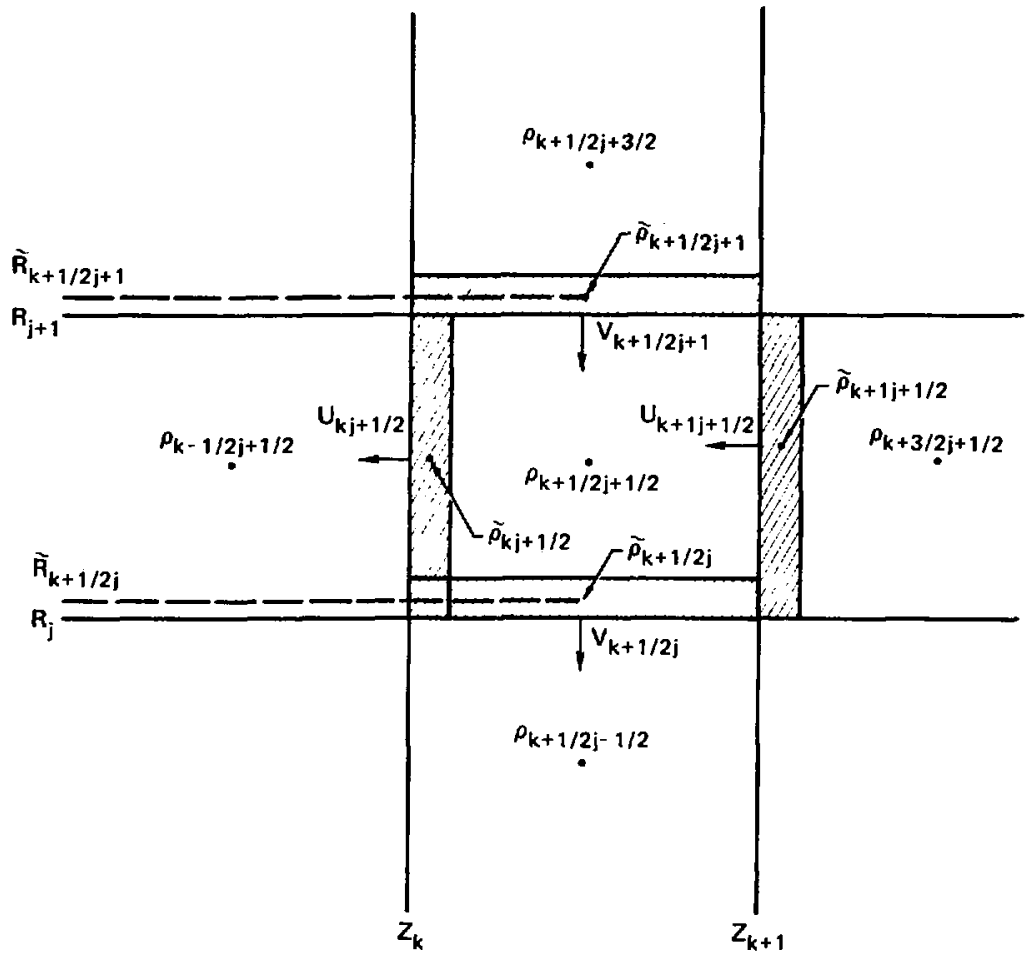

Figure 3.5--Mass is advected by estimating the amount of mass that will pass through zone faces in time $\delta \mathrm{t}$. Advection densities, $\tilde{\rho}$, are computed by Jinearly interpolating to the centers of the advected volumes (hatched regions). 


$$
\begin{aligned}
& \left.A_{k j+1 / 2}^{n+1 / 2}=(R \delta R)\right)_{j+1}^{n+1 / 2}, \\
& A_{k+1 / 2 j}^{n+1 / 2}=R_{k+1 / 2 j} \delta Z_{k+1}^{n+1 / 2} .
\end{aligned}
$$

where

$$
\begin{aligned}
& R_{j+1 / 2}^{n+1 / 2}=R_{j+1 / 2}^{n}+\frac{\delta t}{4}\left(V_{B, j+1}^{n}+V_{B, j}^{n}\right), \\
& \delta R_{j+1 / 2}^{n+1 / 2}=\delta R_{j+1 / 2}^{n}+\frac{\delta t}{2}\left(V_{B, j+1}^{n}-V_{B, j}^{n}\right), \\
& R_{k+1 / 2 j}=R_{j+1 / 2}^{n}-\frac{\delta t}{2}\left(V_{k+1 / 2 j}-V_{B, j}^{n}\right) .
\end{aligned}
$$

The radial fluxes use an interpolated radius, $R$, which corrects for convergence effects near the rotation axis.

Near shock fronts the second-order preseription may evacuate zones and produce negative densilies. This is the physical origin of numerical instabilities. The remedy is to revert to a first-order flux whenever this situation arises. He use the donor cell approximation for the fluxes, wherein the interpolated densities of equation (22), $\tilde{\rho}$, are replaced with donor cell densities, $\rho$ d, given by

$$
\begin{aligned}
& \quad \rho_{k+1 / 2 j}^{d}=\left\{\begin{array}{l}
\rho_{k+1 / 2 j+1 / 2} \\
\rho_{k+1 / 2 j-1 / 2}
\end{array} \text { if }\left(v_{k+1 / 2 j}-v_{8, j}^{n}\right)<0\right. \\
& \text { and similarly for the other three faces. }
\end{aligned}
$$

The new mass density is given by

$$
\rho_{k+1 / 2 j+1 / 2}^{n+1}=\frac{\mu\left(\begin{array}{l}
n+1 \\
k+1 / 2 j+1 / 2
\end{array}\right.}{v_{0} l_{k+1 / 2 j+1 / 2}^{n+1}}
$$

where Volnts is computed from equation (2) using the new grid lines. 


\section{Energy}

Define $\mathcal{E}$ as the tolal internal energy in a zone. Energy is transported consistently with mass by writing

$$
\begin{aligned}
& \mathcal{E}_{k+1 / 2 j+1 / 2}^{n+1}=\sum_{k+1 / 2 j+1 / 2}^{n} \\
& +\left[(\tilde{\varepsilon} \Delta u)_{k+1 j+1 / 2}-(\tilde{\varepsilon} \delta u)_{k j+1 / 2}+(\tau \delta u)_{k+1 / 2 j+1}-(\tilde{\varepsilon} \Delta u)_{k+1 / 2 j}\right] \text {. }
\end{aligned}
$$

where $\tilde{\varepsilon}$ is the specific internal energy of the advected fluid, su. We interpolate on zone centered $\epsilon s$ to find $\tilde{E}$ as follows:

$$
\begin{aligned}
& \tilde{\varepsilon}_{k+1 / 2 j}=\bar{\varepsilon}_{k+1 / 2 j}+\frac{\Delta_{k+1 / 2 j}}{\delta R_{j}} \delta \varepsilon_{k+1 / 2 j}, \\
& \tilde{\varepsilon}_{k j+1 / 2}=\bar{\varepsilon}_{k j+1 / 2}+\frac{\Delta_{k j+1 / 2}}{\delta Z_{k}} \delta \varepsilon_{k j+1 / 2} .
\end{aligned}
$$

In the case the mass fluxes use the donor cell densities, then the donor cell specific internal energies, $\varepsilon^{d}$, defined in an analogous way to $p^{d}$, replace $\tilde{E}$ in equation (29).

The new internal energy density is given by

$$
E_{k+1 / 2 j+1 / 2}^{n+1}=\frac{E_{\substack{n+1 \\ k+1} / 2 j+1 / 2}}{V_{0} 1_{k+1}^{n+1} / 2 j+1 / 2}
$$

\section{Anqular Momentum}

The concern about local conservation of angular momentum in rotating protostar collapse calculations led to the notion of consistent transport (Norman, Wilson, and Barton 1980). Because the relative changes of angular momentum from zone to zone is large near the rotation axis, one cannot ignore changes in A from zone center, to zone boundary. An angular momentum flux is 
calculated by mulitiplying the mass flux of equation (22) by a best guess for the specific angular momentum of the adrected fluid element.

For advection in the radial (R) direction, the specific angular momentum is found by linearly interpolating on the smoothest of three rotational quantities defined in a zone: the angular velocity, the rotational velocity, or the specific angular momentum. Defining of as the total angular momentum in a zone. we write

$$
\begin{aligned}
& d_{k+1 / 2 j+1 / 2}^{n+1}=A N_{k+1 / 2 j+1 / 2}^{n} \\
& +\left[(\operatorname{R\& U})_{k+1 j+1 / 2}-(\operatorname{R\& M})_{k j+1 / 2}+(\operatorname{R\& M})_{k+1 / 2 j+1}-(\operatorname{R\& \mu })_{k+1 / 2 j}\right] \text {, }
\end{aligned}
$$

where $R$ is the specific angular momentum of the advected fluid, su, and is compuled as follows. He define

$$
\begin{aligned}
& K_{k+1 / 2 j+1 / 2}=\frac{A_{k+1 / 2 j+1 / 2}}{\rho_{k+1 / 2 j+1 / 2}}, \\
& W_{k+1 / 2 j+1 / 2}=K_{k+1 / 2 j+1 / 2} \frac{3 R_{j+1 / 2}}{\left(R_{j+1}^{2}+R_{j+1} R_{j}+R_{j}^{2}\right)} . \\
& n_{k+1 / 2 j+1 / 2}=K_{k+1 / 2 j+1 / 2} \frac{2}{\left(R_{j+1}^{2}+R_{j}^{2}\right)} .
\end{aligned}
$$

that is, the values of $W$ and $n$ assuming they are uniform in a zone. Define

$$
\begin{aligned}
& n_{k+1 / 2 j}=n_{k+1 / 2 j}+\frac{\Delta_{k+1 / 2 j}}{\delta R_{j}} \delta n_{k+1 / 2 j}, \\
& w_{k+1 / 2 j}=W_{k+1 / 2 j}+\frac{\Delta_{k+1 / 2 j}}{\delta R_{j}} \delta W_{k+1 / 2 j}, \\
& K_{k+1 / 2 j}=K_{k+1 / 2 j}+\frac{\Delta_{k+1 / 2 j}}{\delta R_{j}} \delta K_{k+1 / 2 j}
\end{aligned}
$$

then 


$$
K_{k+1 / 2 j}=\left\{\begin{array}{llll}
\Omega_{k+1 / 2 j} R^{2}+1 / 2 j & \text { if } & |\delta \Omega / \cap \cdot|_{k+1 / 2 j} & \text { smallest } \\
W_{k+1 / 2 j} R_{k+1 / 2 j} & \text { if } & |\delta W / W \cdot|_{k+1 / 2 j} & \text { smallest } \\
K_{k+1 / 2 j} & \text { if } & |\delta K / K \cdot|_{k+1 / 2 j} & \text { smallest }
\end{array}\right.
$$

The radius factor, $R$, is centered in the advected volume, as defined by equation (26).

For advection in the axial (Z) direction we interpolate on specific angular momentum only:

$$
K_{k j+1 / 2}=K_{k j+1 / 2}+\frac{\Delta_{k j+1 / 2}}{\delta Z_{k}} \delta K_{k j+1 / 2} \text {. }
$$

For the faces where donor cell mass fluxes are used, the donor cell values $\Omega^{d}, W^{d}$, or $K^{d}$ are used in place of the interpolated counterparts.

The new angular momentum density is then

$$
A_{k+1 / 2 j+1 / 2}^{n+1}=\frac{d \begin{array}{c}
n+1 \\
k+1 / 2 j+1 / 2
\end{array}}{v_{0} I_{k+1}^{n+1} / 2 j+1 / 2}
$$

\section{R Momentum}

Because the momentum density components are face-centered, we define momentum zones centered on each component to help visuelize the advection step (the broken grid lines of $f i g, 3.2)$. Figure 3.6 shows the zone for $T_{k+1 / 2 j}$ as we[l as locations of pertinent quantities used for advection of $R$ momentum in both directions. The philosophy remains the same--the $R$ momentum fluxes are computed from mass fluxes and $R$ velocity components averaged to the center of each face. The mass fluxes require some averaging to achieve the proper centering. The face-centered area factors are removed from the $u s$ and are replaced by zone-centered area factors. Define mass fluxes 


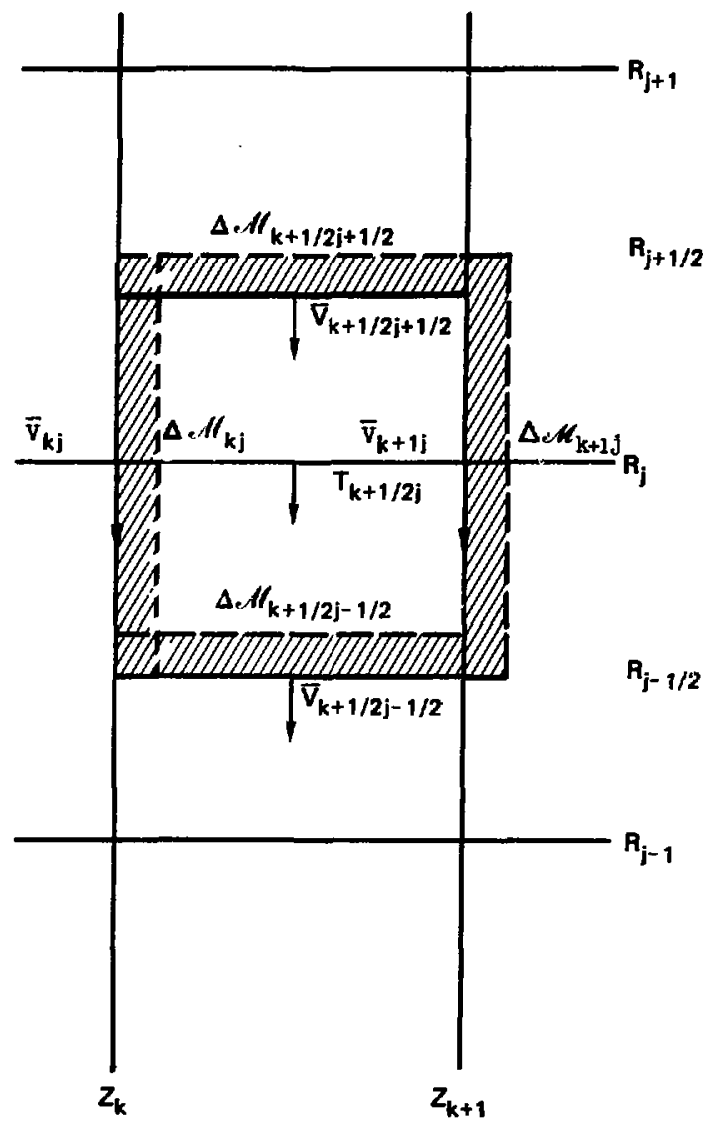

Figure 3.6--Momentum advection requires some averaging because of the staggered grid. R component of momentum is advected by multiplying a mass llux by an $R$ velocity component, both averaged to the face center of the $R$ momentum box. 


$$
\begin{aligned}
& \Delta u_{k+1 / 2 j+1 / 2}=-\frac{1}{2}\left(\frac{\Delta \mu_{k+1 / 2 j}}{R_{k+1 / 2 j}}+\frac{\Delta u_{k+1 / 2 j+1}}{R_{k+1 / 2 j+1}}\right) R_{j+1}^{n+1 / 2} . \\
& \left.\Delta \mu_{k+1 j}=\frac{1 \delta u_{k+1 j+1 / 2}}{2}+\frac{\delta u_{k+1 j-1 / 2}}{A_{k j+1 / 2}^{n+1 / 2}}\right)(R \delta R)_{j}^{n+1 / 2} \text {. }
\end{aligned}
$$

and average $R$ velocity components

$$
\begin{aligned}
& \nabla_{k+1 / 2 j+1 / 2}=\frac{\left(v_{k+1 / 2 j+1}+v_{k+1 / 2 j}\right)}{2}, \\
& \nabla_{k+1 j}=\frac{\left(v_{k+3 / 2 j}+v_{k+1 / 2 j}\right)}{2},
\end{aligned}
$$

where $R$ and $A$ have been previously defined. Then, denoting $g$ the total $R$ momentum component in the zone, we write

$$
\begin{aligned}
& g_{\mathrm{k}+1 / 2 \mathrm{j}}^{n+1}=\mathcal{T}_{\mathrm{k}+1 / 2 \mathrm{j}}^{\mathrm{q}} \\
& +\left[(\nabla \Delta \mu)_{\mathrm{k}+1 / 2 \mathrm{j}+1 / 2}-(\nabla \Delta \mu)_{\mathrm{k}+1 / 2 \mathrm{j}-1 / 2}+(\nabla \Delta M)_{\mathrm{k}+1 \mathrm{j}}-(\nabla \Delta M)_{\mathrm{kj}}\right] .
\end{aligned}
$$

The order of accuracy is set by the mass fluxes, so no special formula is used near shocks.

The new momentum is found by

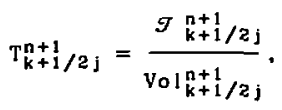

where

$$
V_{0} I_{k+1 / 2 j}^{n+1}=(R \delta R)_{j}^{n+1} \delta Z_{k+1 / 2}^{n+1}
$$

\section{$\underline{\text { Z Momentum }}$}

Figure 3.7 shows the zone for $S_{k j+1 / 2}$, as well as the location of pertinent quantities for advecting $\mathrm{Z}$ momentum in both directions. The area factors do not change with centering. Define mass fluxes 


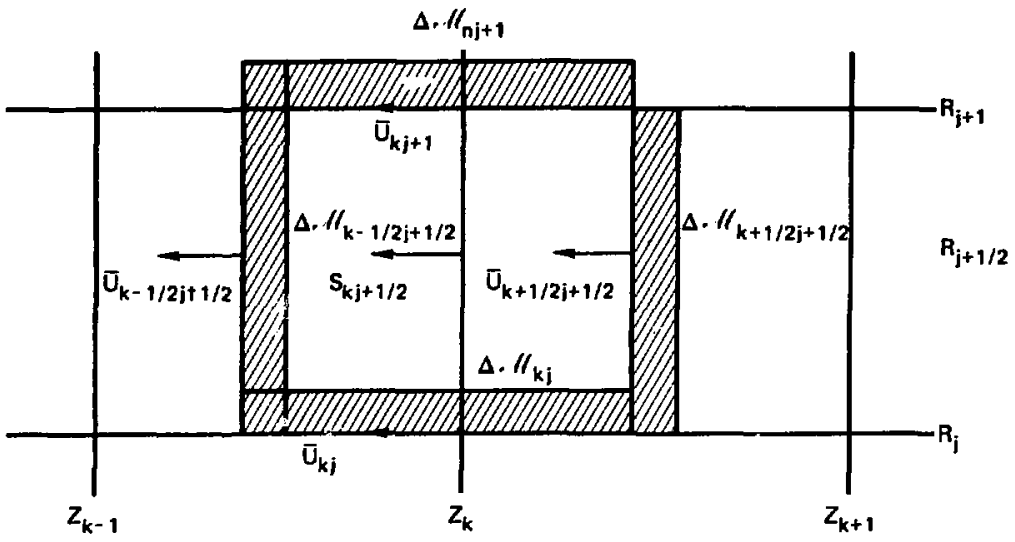

Figure 3.7--Momentum box for the $Z$ component of momentum, plus various averaged mass fluxes and $z$ velocity components. 
31

$\sum_{k+1 / 2 j+1 / 2}=\frac{\left(s u_{k j+1 / 2}+\delta u_{k+1 j+1 / 2}\right)}{2}$

$\Delta u_{k j+1}=\frac{\left(\delta u_{k-1 / 2 j+1}+\delta u_{k+1 / 2 j+1}\right)}{i 2}$,

and average $\mathrm{z}$ velocity components

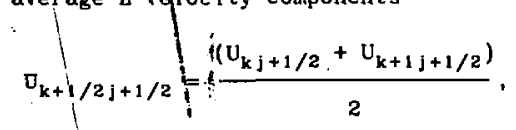

$\mathrm{v}_{\mathrm{k} . \mathrm{j}+1}=\frac{\left(\mathrm{U}_{\mathrm{k}-1 / 2(j+1}+\mathrm{U}_{\mathrm{k}+1 / 2 \mathrm{j}+1}\right)}{2}$.

Then, denoting 5 the tolal $Z$ momentum component in the zone, we writ

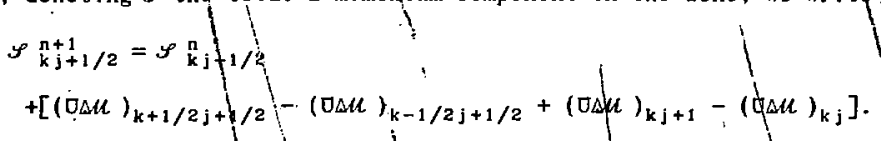
Again, no adjustment heed be made with regard to dọnor cell fluxes tinee $s u$ carries that information.

The new moméntum depsity is found by

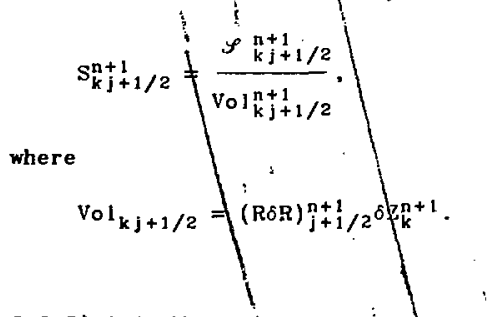

3.2.5) Grid Motion

Grid motion is essentiaj in collapse calculations because otherwise zone resolution would quickly bè lost. In most calculations (those involving collapse to high central densities), the grid moves more or less with the tluid near the center. The $R$ grid motion is separale from the $Z$ grid motion to 
accommodate flattened systems. The basic scheme is to form a new set of grid lines each timestep that satisfies the following requirements:

(1) keep resolution in the center of the cloud

(2) reduce zone to zone transport

(3) maintain zoning regularity

(4) move smoothly

The grid speeds are then derived from the old and new grid lines according to equation (3).

Requirements (1) and (2) are met by compressing the inner zone according to the local velocity convergence:

$$
\begin{aligned}
& \delta \mathrm{Z}_{5 / 2}^{\mathrm{n}+1}=\left(1-f_{\mathrm{Z}}\right) \delta \mathrm{Z}_{5 / 2}^{\mathrm{n}} . \\
& \delta \mathrm{R}_{5 / 2}^{\mathrm{n}+1}=\left(1-f_{\mathrm{R}}\right) \delta \mathrm{R}_{5 / 2}^{\mathrm{n}} .
\end{aligned}
$$

where

$$
\begin{aligned}
& f_{i}=\xi_{z} \sum_{j=2}^{4} \sum_{k=3}^{5} \frac{u_{k j+1 / 2^{\delta t}}}{z_{k}} . \\
& f_{R}=\xi_{R} \sum_{k=2}^{4} \sum_{j=3}^{5} \frac{v_{k+1 / 2 j} \delta t}{R_{j}} .
\end{aligned}
$$

The factors $\xi_{\mathrm{Z}}, \xi_{R}$ are chosen between 0 and 1 . The averaging over nine zones fjlters out noise.

The outer grid line is allowed to move according to need. Basically, three types of boundary motion are used. Using,the radial boundary as an example, they are:

$$
\begin{aligned}
& \text { (a) } R_{J M}^{n+1}=R_{J M}^{n} \\
& \text { (b) } R_{J M}^{n+1}=\left(1-f_{R}\right) R_{J M}^{n} \\
& \text { (c) } R_{J M}^{n+1}=R_{J M}^{n}+\delta t V_{\text {cloud boundary }}
\end{aligned}
$$

Prescription (a) ia used for collapse with a constant volume boundary condition. 
Prescription (b) preserves the zoning ratio at the expense of losing the outer parts of the cloud. This prescription is used for deep collapse which requires very fine central zoning. Since parts of the system are lost, evolutions using prescription (b) are checked against evolutions using prescription (a). Prescription (c) is essential for equilibrium cooling sequences and collapse calulations employing the pressure boundary condition.

Requirenent (3) is met by deriving zoning ratios, $\chi_{z}=\delta Z_{k+3 / 2} / \delta Z_{k+1 / 2}$ and $X_{R}=\delta R_{j+3 / 2} / \delta R_{j+1 / 2}$, such that logarjthmically spaced zones fit between the new first zone and the new outer boundary. For example. if JM2 is the number of active zones between $R_{2}$ and $R_{J W}$, then we have

$$
R_{J M}^{n+1}=\delta R_{5 / 2}^{n+1}\left\{\frac{\chi_{R}^{J M 2}-1}{\chi_{R}-1}\right\} .
$$

Equation $(52)$ is solved for $\chi_{R}$ iteratively using Newton's method.

New zones are generated according to

$$
\begin{aligned}
& \delta \mathrm{R}_{\mathrm{j}+3 / 2}^{\mathrm{n}+1}=\chi_{\mathrm{R}} \delta \mathrm{R}_{\mathrm{j}+1 / 2}, \\
& \mathrm{R}_{\mathrm{j}+1}=\mathrm{R}_{\mathrm{j}}+\delta \mathrm{R}_{\mathrm{j}+1 / 2}, \\
& \mathrm{R}_{\mathrm{j}+1 / 2}=\left(\mathrm{R}_{\mathrm{j}+1}+\mathrm{R}_{\mathrm{j}}\right) / 2, \\
& \delta \mathrm{R}_{\mathrm{j}}=\mathrm{R}_{\mathrm{j}+\mathrm{I} / 2}-\mathrm{R}_{\mathrm{j}-1 / 2} ; \mathrm{j}=2,3, \ldots . \mathrm{JMM},
\end{aligned}
$$

and similarly for the $\mathrm{Z}$ zones.

Requirement (4) is met by allowing the grid speeds to change by no more than 30 percent in a timestep.

\subsection{6) Grayitational Potential}

The deliberations of sections 3.2 .1 to 3.2 .5 advance the cloud and grid to timelevel $n+1$, ending the hydrodynamic part of the calculation. The solution 
technique for the Poisson equation used here has been described by Black and Bodenheimer (1975), but will be repeated here. The plan is to find the steady-state solution to the diffusion equation

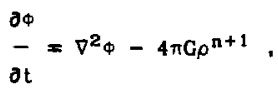

using the ADI method for a series of iterative "timesteps". The time in this equation bears no relation to the physical time of the evolution; the timesteps are chosen to speed convergence.

Let $\phi^{P}$ be the $p^{t h}$ estimate for the gravitational field, and $\delta t^{p}$ be the timestep for the $p^{\text {th }}$ iteration. Defining

$$
\begin{aligned}
& \delta \Phi_{k j+1 / 2}=\Phi_{k+1 / 2 j+1 / 2}-\Phi_{k-1 / 2 j+1 / 2}, \\
& \delta \Phi_{k+1 / 2 j}=\Phi_{k+1 / 2 j+1 / 2}-\Phi_{k+1 / 2 j-1 / 2} .
\end{aligned}
$$

the field estimate $\Phi^{P}$ is advanced to $\Phi^{\mathrm{P}+1}$ by the two-step ADI procedure:

$$
\begin{aligned}
& \frac{\left(\phi^{P+1 / 2}-\phi^{P}\right)_{k+1 / 2 j+1 / 2}}{\delta t^{p}}=\left(\frac{\delta \Phi P_{k+1 j+1 / 2}}{\delta Z_{k+1}}-\frac{\delta \Phi P_{j+1 / 2}}{\delta Z_{k}}\right) / \delta Z_{k+1 / 2} \\
& +\left(R_{j+1} \frac{\delta \Phi p_{k+1}^{+1 / 2} 2_{j+1}}{\delta R_{j+1}}-R_{j} \frac{\delta \phi \mathrm{k}^{+1}+1 / 2 j}{\delta R_{j}}\right) /(R \delta R)_{j+1 / 2}-4 \pi \rho_{k+1 / 2 i+1 / 2}^{n+1} \text {, }
\end{aligned}
$$

then

$$
\begin{aligned}
& \frac{\left(\phi^{\mathrm{P}+1}-\phi^{\mathrm{P}+1 / 2}\right)_{k+1 / 2 j+1 / 2}}{\delta \mathrm{L}^{\mathrm{P}}}=\left(\frac{\delta \phi^{\mathrm{P}+1} \mathrm{k}+1 \mathrm{j}+\mathrm{l} / 2}{\delta \mathrm{Z}_{\mathrm{k}+1}}-\frac{\delta \phi^{\mathrm{P}+1} \mathrm{kj+1/2}}{\delta \mathrm{Z}_{\mathrm{k}}}\right) / \delta \mathrm{Z}_{k+1 / 2} \\
& +\left(R_{j+1} \frac{\delta \Phi \mathrm{k}_{k+1 / 2 j+1}^{+1 / 2}}{\delta R_{j+1}}-R_{j} \frac{\delta \Phi \mathrm{p}_{\mathrm{k}+1 / 2}^{+1 / 2} \mathrm{j}}{\delta R_{j}}\right) /(R \delta R)_{j+1 / 2} \\
& -4 \pi \mathrm{G} \rho_{k+1}^{n+1} / 2 j+1 / 2, \quad p=0,1, \ldots \ldots \beta-1 \text {. }
\end{aligned}
$$

The implicjt sweeps are solved by the method of backward substitution described by Richtmeyer and Morton (1967). The interior boundary conditions are 
set by symmetry, and the exterior boundaryconditionsare set by a multipole expansion as detailed in section 2.3 .

The iteralive $t$ imesteps, $\delta t^{p}$, are defined

$$
\delta \mathrm{tP}=\alpha^{\mathrm{P} \delta t_{\text {max }}} ; \mathrm{P}=0,1 \ldots \ldots-1 \text {. }
$$

where

$$
\delta \mathrm{t}_{\max }=\max \left(Z_{K M}^{2}, R_{J M}^{2}\right) / 4 .
$$

and

$$
\begin{aligned}
& \alpha=\left(\delta \mathrm{t}_{\min } / \delta \mathrm{t}_{\max }\right)^{1 /(\beta-1)} . \\
& \delta \mathrm{t}_{\min }=\min \left(\delta \mathrm{Z}_{5 / 2}^{2}, \delta \mathrm{R}_{5 / 2}^{2}\right) / 4 .
\end{aligned}
$$

Typically $\beta=J M / 2$ is adequate for convergence. The timestep prescription is chosen in analog to a treatment by Peaceman and Rachford (1955), who solved diffusion in a square. rectangular mesh. The timesteps are chosen to reduce the amplification factor of equations $(56,57)$ for modes of wavelength comparable to $\left(\delta t^{\mathrm{P}}\right)^{1 / 2}$. The new potential is given by $\phi^{\mathrm{n}+1}=\phi^{\hat{B}}$.

\subsection{7) Interfaces}

Materials of different properties in contact are handled through an interface method. The boundary between two materials is called an interface. In the present application, an interface separates the hot intercloud medium trom the cool cloud phase. This provides a physically realistic pressure boundary condition in that the cloud boundary reacts to forces acting on it. This feature is essential to constructing rotating, isothermal equilibria, whose outer boundary is free (Chapler 4 ).

Operationally, each material in the calculation is labeled. The label is used as an indicator of material properties, e.g., equation of state. A mixed zone is a zone containing more than one material. The clean zones are advanced 
us described in sections 3.2 .1 to 3.2.5. Therefore, a prescription for how to advance mixed zone is the only further information needed. The following treatment of mixed zones has been developed by Leblanc based on work by deBar (1974). The actual lines constituting the interfaces are not stored, but only information on how they divide mixed zones into fractional parts. We store the following quantities for each material i present in a mixed zone:

$$
\begin{aligned}
& v_{t}^{i}=v_{0} l^{i} / \text { Vol , tractional volume, } \\
& D_{f}^{i}=J^{i} v_{i}^{i} \text {, fractional density. } \\
& E_{f}^{l}=D_{f}^{l} \varepsilon^{j} \text {, fractional internal energy density, } \\
& A_{l}^{j}=D_{i}^{i} K^{i} \quad \text {, fractional angular momentum density. }
\end{aligned}
$$

Here vol is the volume of the zone, and $v_{0} l^{i}, p^{i}, \varepsilon^{i}$, and $K^{i}$ are the volume, density, specific internal energy, and specific angular momentum of material i. The quantities $D_{\mathfrak{l}}^{i}, E_{\mathfrak{l}}^{i}$, and $A_{\mathfrak{l}}^{i}$ are iherefore the densities material $i$ would have jf it were spread out over the entire zone. From these definitions we have obviously

$$
\begin{aligned}
& 1=\Sigma V_{f}^{i} \\
& \rho=\Sigma D_{f}^{i} \\
& E=\Sigma E_{f}^{i} \\
& A=\Sigma A_{f}^{i} .
\end{aligned}
$$

The pressure in a mixed zone is found by adding the partial pressures:

$$
P=\Sigma\left(\gamma^{i}-1\right) E_{f}^{i}
$$

The angular velocity of a mixed zone is defined as a mass-weighted average of the fractional angular velocities. Thereafter mixed zones are accelerated like clean zones. 
Compressional heating trom the artificial viscosity and PDV work calculations are partitioned equally to each material i in a mixed zone:

$$
\begin{aligned}
& E_{f}^{j \cdot q}=\frac{E^{q}}{E}-E_{l}^{i} . \\
& E_{f}^{j} \cdot P=\frac{E^{P}}{E^{q}}
\end{aligned}
$$

The motion of the interface comes about by advecting the fractional volumes along with the other fractional densities. As before, advection is done in 10 sweeps, however the donor cell scheme is used for advection to and from mixed zones. Consider a triad of zones containing at least one mixed zone (figure 3.8), and suppose we wish to update the second zone. Define $\mathscr{F}_{\mathrm{L} . \mathrm{R}}^{\mathrm{j}}$ as the fractional flux on the left;right, and $A_{L, R}$ the areas of the zone faces on the left,right. Mass, energy, and momentum are advected identically, so for illustration we will interpret $\mathcal{F}$ as a mass flux. We write

$$
F_{L, R}^{i}=\Delta_{L, R}^{i}\left(D_{f}^{i} / V_{l}^{i}\right)_{d}
$$

where d refers to the donor cell, and $\Delta_{L, R}$ are chosen according to three cases:

\section{Clean to Mixed}

Referring to $t$ igure $3.8 \mathrm{~b}$, for $f$ low from a clean zone to a mixed zone, we have simply

$$
\Delta^{\prime}=\left(V-V_{t}\right) \delta \dot{t}_{i} t
$$

The donor cell remains clean and the acceptor cell remains mixed. 
Figure 3.8

(1)

(2)<smiles>CCCCC</smiles>

(3)

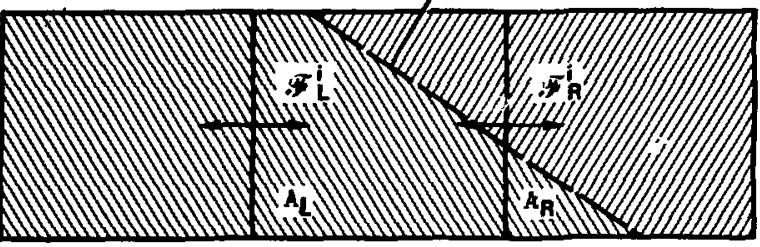

(b) Cleen to mixed

(2)

(3)

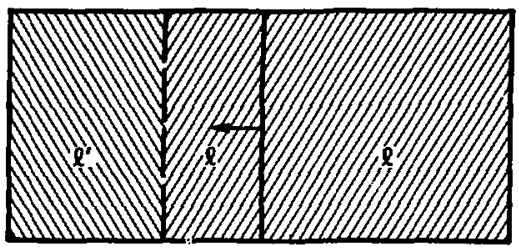

(2)

(3)
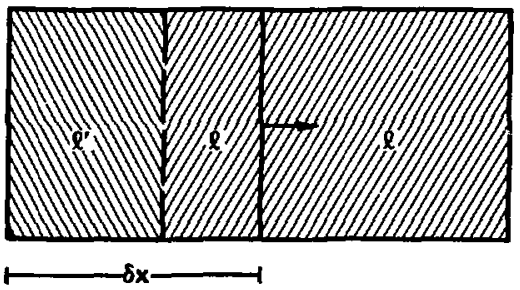

(2)

(3)

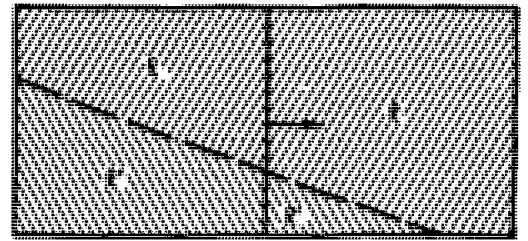

(d) Mixed to mixed 
Figure 3.B-Advection in a triad of zones containing at least one mixed zone. (a) The interface between two fluids has no separate reality other than how it divides the zones into tractional volumes. Advecting in ID sweeps, the fractional fluxes, $\mathcal{F}^{\mathfrak{j}}$, are derived according to three interface geometries: (b) clean to mixed zone advection. (c) mixed to clean zone advection, and (d) mixed to mixed zone advection. 


\section{Mixed to Clean}

Referring to figure $3.8 \mathrm{c}$, we see that material $l$ may become negative in the donor cell if $\left|\mathrm{V}-\mathrm{V}_{\mathrm{a}}\right| \delta \mathrm{t}>\left(\mathrm{v}_{t}^{\ell} \delta \mathrm{X}\right)_{\mathrm{d}}$. Therefore

$$
\Delta^{i}=\operatorname{sgn}\left(v-v_{z}\right) \cdot \min \left[\left|V-v_{t}\right| \delta t,\left(v_{i}^{l} \delta x\right)_{d}\right] \delta_{i} l \cdot
$$

It $\left|V-V_{f}\right| \delta t>\left(V_{f}^{l} \delta x\right)_{d}$, then we take the next material in line acoording to

$$
\Delta^{i}=\operatorname{sgn}\left(V-v_{e}\right) \cdot \min \left[\left|V-v_{g}\right| \delta t-\Delta^{l},\left(v_{l}^{l} \delta x\right)_{d}\right] \delta_{l} l .
$$

and so on until everything $\left|V-v_{8}\right| \delta t$ behind the flow has been taken. Thus, the donor cell may become clean and the acceptor cell may become mixed.

\section{Mixed to Mixed}

$$
\begin{aligned}
& \text { We define apertures, } A^{i} \text {, through which material } i \text { may pass as follows: } \\
& A^{i}=\left(V_{\mathrm{f}, L}^{i}+V_{\mathrm{f}, \mathrm{R}}^{i}\right) / 2 \text {. }
\end{aligned}
$$

then

$$
\Delta^{i}=\left(V-V_{g}\right) \delta L^{i} \text {. }
$$

Notice that $\Sigma d^{i}=1$, so that the scheme is conservative.

The difference equations for the advection of the fractional densities and Iractional volusnes are

$$
\begin{aligned}
& \mathrm{v}_{\mathrm{i}}^{\mathrm{i}, \mathrm{n}+1}-\mathrm{v}_{\mathrm{f}}^{\mathrm{i} \cdot \mathrm{n}}=-\frac{\left(\Delta_{\mathrm{R}}^{\mathrm{i}} A_{\mathrm{R}}-\Delta_{\mathrm{L}}^{\mathrm{i}} A_{\mathrm{L}}\right)}{V_{\mathrm{ol}}},
\end{aligned}
$$

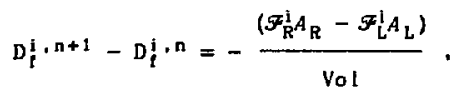

and analogously for $E_{f}^{i}$ and $A_{f}^{j}$. Total densities are found by summing the fractional densities. The fractional volumes are normalized sa they once again sun to unity. 
Finally, integrated mass fluxes are computed for use in the linear momentum advection calculation:

$b U=A \Sigma F^{i}$.

\section{3) Stability}

The final operation in the problem cycle is the calculation of a new timestep to be used in the next cycle. Explicit hydrodynamics requires the timestep to satisfy the Courant condition for stability, which for a one dimensional problem is

$$
\delta \mathrm{t} \in \frac{\delta \mathrm{x}}{(c+|u|)}
$$

where $\delta \mathrm{X}$ is the zone size, $C$ is the local sound speed, and $u$ is the bocal fluid velocity relative to the grid. We calculate three timesteps for each zone:

$$
\begin{aligned}
& \delta \mathrm{t}_{1}=\frac{\min (\delta \mathrm{R}, \delta \mathrm{Z})}{c} . \\
& \delta \mathrm{t}_{2}=\frac{\delta \mathrm{Z}}{\left|\mathrm{U}-\mathrm{U}_{\mathrm{g}}\right|} . \\
& \delta \mathrm{t}_{3}=\frac{\delta \mathrm{R}}{\left|\mathrm{V}-\mathrm{v}_{\mathrm{g}}\right|} .
\end{aligned}
$$

The artificial viscosity also limits the timestep, since $Q^{Z Z}$ and $Q^{R R}$ are used to form a momentum diffusion prablem. For an explicit diffusion scheme the timestep is limited by

$$
\delta t \leqslant \frac{\delta x^{2}}{4 y} .
$$

where $y$ is the kinematic viscosity. A comparison of equation (13) to the Navier-Stokes equation shows the numerical kinematic viscosity to be 


$$
\begin{aligned}
& \nu_{\mathrm{ZZ}}=\delta \mathrm{Z}(\mathrm{qQ} \mathrm{ZZ} / \rho)^{\mathrm{l} / 2}=\mathrm{q} \mid \delta \mathrm{U} / \delta \mathrm{Z} \\
& \nu_{\mathrm{RR}}=\delta \mathrm{R}(\mathrm{qQRR} / \rho)^{1 / 2}=\mathrm{q}|\delta \mathrm{V}| \delta \mathrm{R} .
\end{aligned}
$$

thus we define another timestep for zones with nonzero $Q$,

$$
\delta \mathrm{t}_{4}=\min \left(\frac{\delta \mathrm{Z}}{4 \mathrm{q}|\delta \mathrm{U}|} \cdot \frac{\delta \mathrm{R}}{4 \mathrm{q}|\delta \mathrm{V}|}\right)
$$

The new timestep is then

$$
\delta \mathrm{t}^{\mathrm{n}+1}=b\left(\frac{1}{\delta \mathrm{t}_{1}{ }^{2}}+\frac{1}{\delta \mathrm{t}_{2}{ }^{2}}+\frac{1}{\delta \mathrm{t}_{3}{ }^{2}}+\frac{1}{\delta \mathrm{t}_{4}{ }^{2}}\right)^{-1 / 2} .
$$

where $b$ is the safely factor, $0<b \leqslant 1$. Usually $b=1 / 2$ in the protostar calculations.

The timestep is limited to less than a 30 percent increase per cycle to maintain accuracy when the system makes abrupt dynamical changes.

\section{4) Accuracy}

In this section we discuss the accuracy of the potential solver and the conservation properties of the hydrodynamics. The overall accuracy of the code is determined by evolving test systems whose solutions are known, and that are closely related to the system under study. In addition, several diagnostics for the local conservation of angular momentum and the accuracy of the equilibria are described.

\subsection{1) Gravitational Potential}

The accuracy of the gravitational potential is determined by the convergence factor 


$$
x=\frac{\left|\nabla^{2} \phi-4 \pi G \rho\right|}{4 \pi G \rho} .
$$

which, acc.rding to Black and Bodenheimer (1975), should be $<10^{-5}$ to acheive potential gradient accuracy of about a percent. The convergence $f$ actor is found to be typically between $10^{-6}$ and $10^{-10}$ in these calculations, with the better accuracy at the center of the cloud.

\subsection{2) Hydrodynamics: Conservation}

Mass, I inear momentum, and angular momentum are globally conserved because transport uses fluxes. This is easily seen by summing. for example, equation (21) over all $k, j$ and noticing that the fluxes cancel in pairs. Therefore $\mu^{n+1}$ $=\mu^{n}$, assuming no mass llow across the boundary of the grid. Internal energy is advected conservatively, however the PDV work term is not conservative as differenced. In practice total energy is conserved to about 5 percent, due partly to the work term, and partly to the staggered arrangement of the velocity components (the total energy for a zone can be defined in several ways depending on how the velocity components are averaged).

Local conservation comes from doing fluid dynamics correctly on a zonal level. For instance. fluid elements must carry local intrinsic properties such as linear and angular momentum, temperature, magnetic flux, etc. The present code locally conserves in that sense because fiuxes of momentum, angular momentum, and energy are constructed from mass fluxes. Local conservatica therefore relies on the accuracy of the mass fluxes and the intensive quantities $\tau, \pi, \nabla, \nabla$, which multiply them (sec. 3.2.4). These quantities are second-order accurate, except near shock tronts where they are replaced by first-order accurate quantities. 
The local conservation of angular momentum is monitored by changes in the specific angular momentum spectrum, defined by

$$
\mu(K)=\int_{0}^{K} \mathrm{~d}(\mu(k)
$$

where $K=n R^{2}$, and duik) is the mass at specific angular momentum $k$. $\mu(K)$ is a constant of the motion for ideal, isothermal flow, therefore any changes in the computed spectrum show numerical redistribution of engular momentum. Figure 3.9 shows spectra take it several stages in the collapse of case 5B (chap. 5 ). Numerical diffusion is responsible for slight changes from the theoretical spectrum, but is mi.:or because of the tiny mass irvolved. The deviation for the bulk of the cloud is minimal. For a more complete discussion of numerical diffusion and an llustration of the effects, see Norman, Wilson, and Barton $(1980)$

\subsection{3) Rotating Equilitrium}

A demonstration of the abjlity lo generate rotating equilibria hydrodynamically is given in appendix A. A sequence of rotating polytropes are calculated corresponding to the sequence designated $\left(n=1.5, n^{*}=0\right)$ by Bodenheimer and Ostriker (1973). Our results agree to roughly 10 percent.

The accuracy of rotating isothermal equilibria is determined by the convergence factor

$$
x^{\prime}=\frac{\left|a^{2} \nabla^{2} \ln \rho+s\right|}{|s|} .
$$

where $a$ is the isott.ermal sound speed, and 


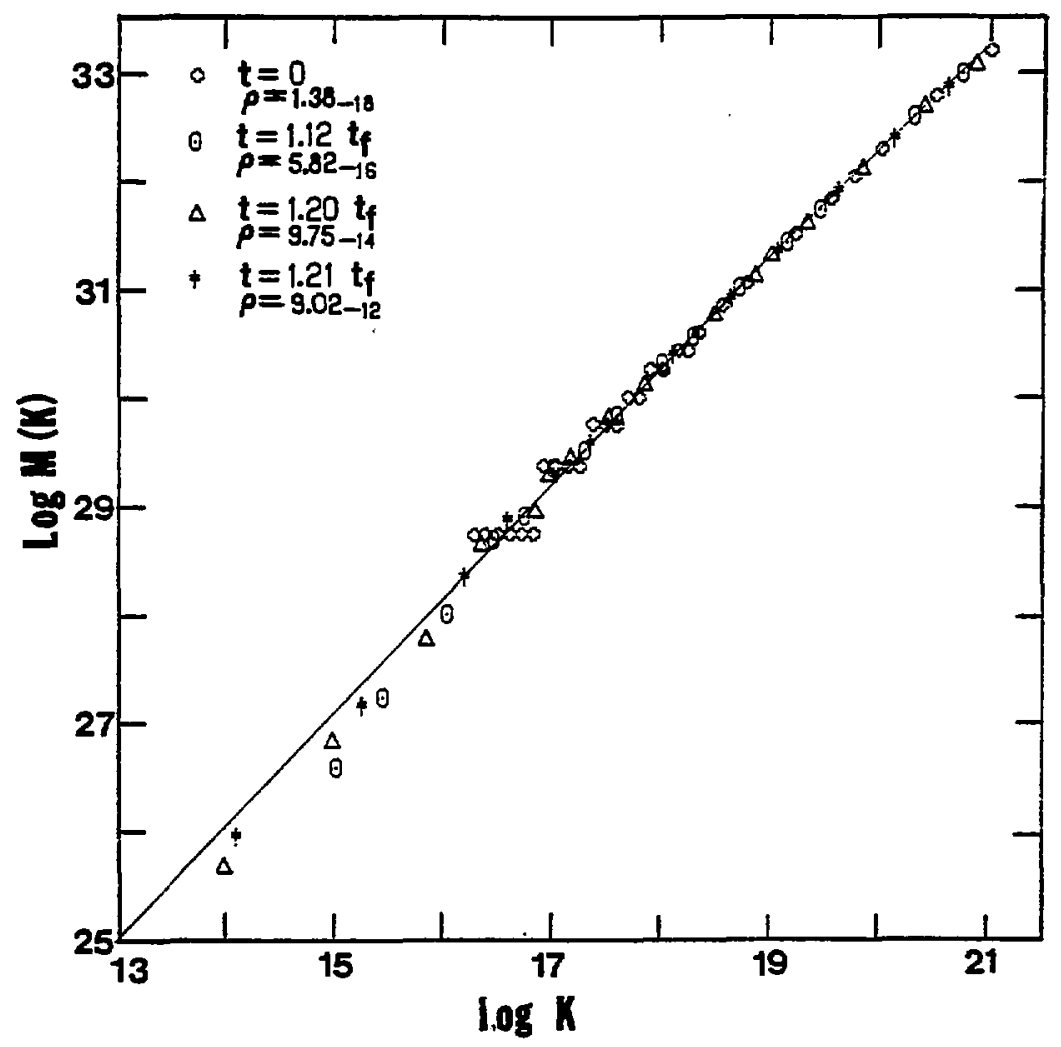

Figure 3.8--Specific angular momentum spectrum, as defined by equation (B3), from evolution 5B (chap. 5). Deviations from the initial spectrum (solid line), indicate numerical redistribution of angular momentum. This evolution has done quite well. 


$$
s=-4 \pi \mathrm{G} \rho+\frac{1 \Delta}{R \Delta R}
$$

$a^{2} \nabla^{2} l n \rho+=0$ is a Lane-Enden equation generalized to include rotation, and with appropriate boundary conditions gives $\rho$ in equilibriun. $x^{\prime}$ is typically 5 percent in the equilibriun calculations. 
CHAPTER 4

ROTATING ISOTHERYAL EQUILIBRIUM

\section{1) Problem Statement}

The picture of a cold, dense, isothermal cloud in pressure equilibrium with a hot intercloud medium is a paradigm for the interstellar medium. We seek the solution to the following equilibrium problem: given an isothermal cloud in a state of permanent ratation ${ }^{1}$ characterized by mass $\mu$, sound speed $a$, angular momentum $d$, and angular momentum distribution function $s(\tau)$ i restrained by an external pressure $P_{x}$; then what are the accessible states of equilibrium? Here the effects of $J$ and $s(r)$ on structure are of particular interest regarding $r i n g$ and disk structure. The conditions under which no equilibrium is possible is of interest because this gives the criterion for gravitational collapse. Also, overstable equilibria are of interest because they are plausible initial conditions for collapse, and attractive from the point of $v i e w$ that they are well defined.

The equilibrium problem in the absense of ratation has been solved by Bonner (1956), and Ebert (1955). The spherically symmetric solutions are justifiably called Bonner-Ebert spheres, and their properties have been reviewed by Shu (1977). Equilibrium is possible when

$$
\mu<\mu_{c}=1.18 \frac{a^{4}}{\left(G^{3} P_{x}\right)^{1 / 2}} .
$$

1 Tassoul (1978) defines a system in permanent rotation as being isolated, stationary. frictionless, and free of electromagnetic forces. 
A cloud with $\mu>\mu_{c}$ will undergo gravitational collapse, which may be initiated by some mechanism that increases the surface pressure or decreases the cloud temperature.

In this chapter a set of rotating analogs to Bonner-Ebert spheres are described which are in eneral differentially rotating. Both ring and disk equilibria are found. In addition an empirical line of criticality to collapse is given. An experiment testing the stability of a disk equilibrium to axisymmteric perturbations is also described.

\section{2) Procedure}

Ideally one would like to tind equilibria by solving the governing equilibrium equation,

$$
a^{2} \nabla^{2} \operatorname{ln\rho }=-4 \pi \mathrm{G} \rho+\nabla \cdot \underline{C} .
$$

where $C=\Omega^{2} R t_{R}$, the centrifugal acceleration. Equation (2) follows from the hydrostatic equations and the Poincare-Wavre theorem, which states that $\Omega=\Omega(R)$ for barotropic stars (Tassout 1978). The solution of equation (2) has not been atlempted here because of the difficulty of applying the outer boundary condition, $\rho=$ constant, on a free boundary.

Instead, equilibrium is tound hydrodynamically. Although not a strict equilibriun because of residual waves of small amplitude, the equilibrium convergence factor $\chi^{\prime} \sim 5 \cdot 10^{-2} \quad\left(\chi^{\prime}\right.$ is defined in sec. 3.4.3). The procedure is to relax an input configuration (uniform density sphere with some linear rotation (aw) to equilibrium by adding a damping term to the equation of motion:

$$
\left.\frac{\partial \rho x}{\partial \mathrm{t}}\right)_{\text {dampint }}=-f \frac{\rho y}{\tau_{\mathrm{d}}} .
$$


where the damping time $\tau_{d}$ is chosen to be the sound-crossing time for the cloud, and $f$ is typically 1 or 2 . The constant pressure boundary condition is applied by maintaining hot intercloud gas exterior to the cloud. The two gases are separated by an interface (see sec. 3.2.7). Once equilibrium is reached, the cloud temperature is slowly decreased according to

$$
T=T_{0} \exp \left(-f^{\prime} t / \tau_{d}\right) \text {. }
$$

where $f^{\prime}$ is typically 0.1 or 0.2 . As the cloud cools, a continum of equilibria are generated. The sequence tcrminates with the onset of gravitational collapse which is calculated without the damping term present.

The parameters for the spheres that generate the head of each cooling sequence are given in table 4.1. Rotation laws are given by

$$
\Omega(R)=\Omega_{c}[1+(s-1)-j \text {. }
$$

where $R$ is the cylindrical radius, $Q_{c}$ is the central angular velocity, and $s$ is the ratio of the angular velocity at the edge of the cloud to $n_{c}$. By restricting the angular momentum distribution to equation (5), we reduce the free function $s(x)$ to a free parameter, s. 
TABLE 4.1

COOLING SEQUENCE PARAMETERS

$$
\begin{aligned}
& \text { Mass: } \mu=1 \mu_{0} \\
& \text { Radius: } R_{0}=8.61 \cdot 10^{i 7} \mathrm{~cm} \\
& \text { Sound Speed: } a=2 \cdot 10^{4} \mathrm{~cm} \mathrm{~s}^{-1} \\
& \text { External Pressure: } P_{x}=3.11 \cdot 10^{-13} \text { dyne } \mathrm{cm}^{-2}
\end{aligned}
$$

\begin{tabular}{cccc} 
Sequence & $J\left(10^{54} \mathrm{~g} \mathrm{~cm} \mathrm{~s}^{-2}\right)$ & $\mathrm{s}$ & Zones \\
\cline { 2 - 2 } & 2.72 & - & $42^{2}$ \\
$4 \mathrm{~B}$ & 4.72 & 1 & $22^{2}$ \\
$4 \mathrm{~B}^{\circ}$ & 4.72 & 1 & $22^{2}$ \\
$4 \mathrm{~B}^{\prime}$ & 4.72 & 10. & $22^{2}$ \\
$4 \mathrm{C}$ & 6.08 & 0.1 & $42^{2}$ \\
$4 \mathrm{D}$ & 7.22 & 1 & $22^{2}$ \\
$4 \mathrm{E}$ & 1.65 & 1 & $42^{2}$ \\
$4 \mathrm{~F}$ & 10.65 & 1 & $22^{2}$
\end{tabular}




\section{3) Scaling and Parameterization}

The scale given in table 4.1 is chosen for reference only, as the problem scales. Two useful paraneters for scaling are

$$
\eta=\frac{\mu}{\mu_{c}} \quad \ldots \mu_{c}=\frac{1.18 a^{4}}{\left(G^{3} P_{x}\right)^{1 / 2}}
$$

and

$$
x=\frac{J}{J_{c}}, J_{c}=\frac{\Delta^{7}}{\mathrm{G}^{2} \mathrm{P}_{\mathrm{x}}} .
$$

We can write

$$
x=1.34 J\left(\frac{P_{x}}{G^{5}}\right)^{t / 0}{ }_{(-)^{7 / 4}}^{\eta} .
$$

therefore along a cooling sequence $\chi=\chi(\eta)$ since $M, J$, and $P_{x}$ are held constant. Each cooling sequence was started at $\eta=0.1$ to ensure an accesible equilibrium. The parameter set $(\eta, x, s)$ will turn out to be useful for defining the critical line in section 4.5 .

An alternate set of parameters based on energy ratios is also given because of its widespread use, in the literature on rotating polytropes. A rolating isothermal cloud in equilibrium satisfies the virial theorem (Spitzer 1978)

$$
0=2 K+w+30-\int P_{x} r \cdot d g .
$$

where

$$
\begin{aligned}
& x=-\int_{2}^{1} d^{3} r \rho \Omega^{2} R^{2} . \\
& W=-\int d^{3} r \rho r \cdot \nabla \phi . \\
& \infty=\int d^{3} r P .
\end{aligned}
$$

and $P_{X}$ is the external pressure. The surface term distinguishes isolhermal 
equilibria from other barotrapic equilibria gyatems (stars). Defining two parameters

$$
\begin{aligned}
\alpha & =\frac{300}{2 m} . \\
\beta & =\frac{x}{m} .
\end{aligned}
$$

the ratios of thermal and rotational energy to the magnitude of the gravitational energy, then equation (9) becomes

$$
1=2(\alpha+\beta)+\frac{\int P_{x} x \cdot d a}{M} .
$$

The parameters $(\alpha, \beta, s)$ are used in the following because the classical instabilities (bar, ring) are best known by their critical values of $\beta$.

\section{4) Results}

Figure 4.1 shows the 1rajectories of the eight cooling sequences in $(\alpha, \beta)$ parameter space. At the top of each track gravitational energy is inly a fraction of the internal energy of the cloud. As the temperature is lowered the cloud's position moves downward and to the right. The cloud flattens as the rotational energy becomes more important, however in the case of $4 \mathrm{~B}^{\prime \prime}$, the cloud turns inlo a ring. The sequences stop at the points where collapse sets in. The fiducials mark $\eta$ as defined by equation ( $(6)$.

It is difficult in practice to locate precisely where the sequence becomes dynamic. The sequence designated $s$ followed a sequence of Bonner-Ebert spheres. The computed position of the critical Bonner-Ebert sphere is in error by 15 percent, and may be taken as a rough error estjmate for the other transition points. No clear transition was seen for sequence $4 F$. The system was so flat 


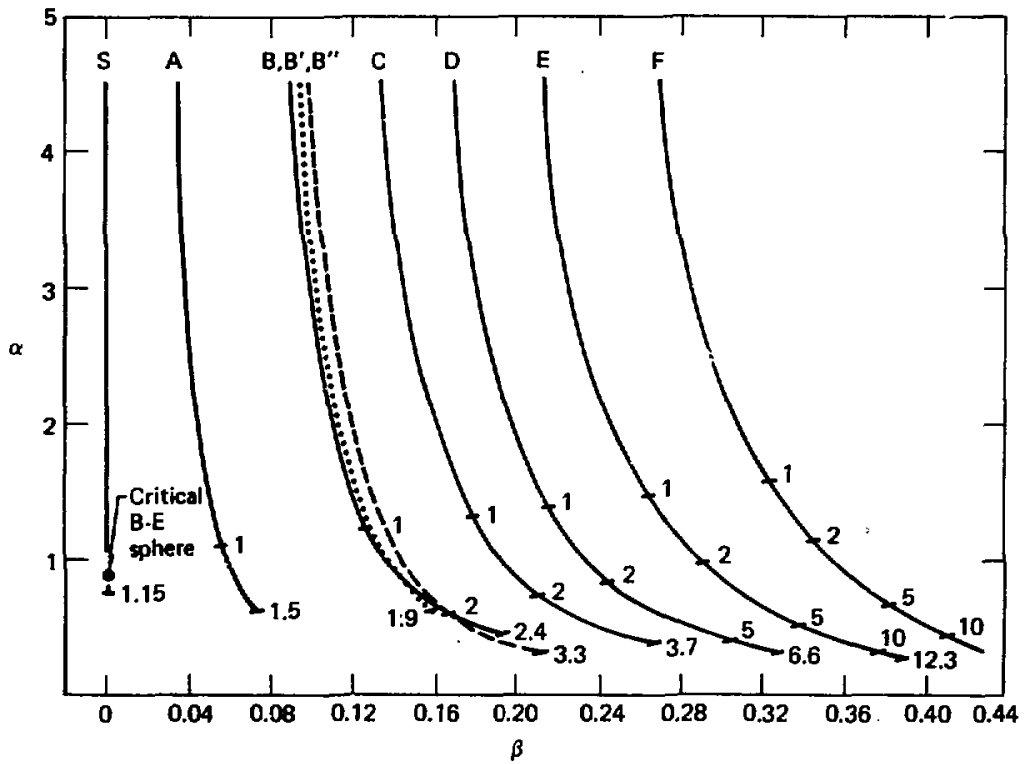

Figure 4.1-Cooline tracks in $(\alpha, \beta)$ space for isothermal, equilibrium spheroids. Sequences begin at large a (high temperature), and descend until collapse sets in. Fiducials mark the number of critical Bonner-Ebert sphere masnes. Bonner-Ebert spheres lollow sequence $S$, run as a check. 
at the bottom of the track that the zoning was inadequate (22 zones). Sequence $4 E$ was run with $42^{2}$ zones and a clear transition was seen. The critical line is examined turther in section 4.5. Table 4.2 gives the values of $(\alpha, \beta)$ and $(\eta, x)$ at criticality. The dimensional soundspeed is included for reference.

TABLE 4.2

CRITICA' 'S THERMAL SPHEROID PARAMETERS

$\begin{array}{cccccc}\text { Sequence } & \alpha & \beta & \eta & \chi & \text { Soundspeed }\left(10^{4} \mathrm{~cm} \mathrm{~s}^{-1}\right) \\ & - & - & - & - & 1.59 \\ 4 \mathrm{~A} & 0.61 & 0.073 & 1.5 & 0.3 & 1.43 \\ 4 \mathrm{~B} & 0.44 & 0.194 & 2.4 & 1.3 & 1.28 \\ 4 \mathrm{~B}^{\circ} & 0.62 & 0.159 & 1.9 & 0.9 & 1.51 \\ 4 \mathrm{~B}^{\circ} & 0.31 & 0.215 & 3.3 & 2.3 & 1.24 \\ 4 \mathrm{C} & 0.37 & 0.228 & 3.7 & 3.5 & 1.11 \\ 4 \mathrm{D} & 0.31 & 0.313 & 6.6 & 11.6 & 0.97 \\ 4 \mathrm{E} & 0.23 & 0.385 & 12.3 & 36.7 & \ldots \\ 4 \mathrm{~F} & \ldots & \ldots & \ldots & \ldots & \ldots\end{array}$


Several meridional sections for selected sequences are shown in $\mathrm{f}$ igures 4.2 to 4.8. Isodensity, or equivalently, isopotential contours are displayed for one quadrant of the cloud. The rotation axis is horizontal. The last picture in each figure is close to the transition model. Notice particularly the large elfect of angular momentum distribution in sequences $4 B, 4 B^{\circ}$, and $4 B^{\prime \prime}$. Figure 4.5 shows the $s=0.1$ model, which becomes a ring as the internal energy of the cloud is reduced. Previous thinking was that a ring would be produced when $\beta$ 0.36 based on Bardeen's analysis of axisymetric instability of Maclaurin spheroids. While this may be a sufficient condition tor ring formation, it is shown here to be not necessary since $\beta \leqslant 0.21$ for sequence $4 B^{\prime}$.

The distributions of density and angular velocity for the transition models are shown in $f$ igures 4.9 and 4.10 , respectively. Whereas $\rho(r)$ approaches $r^{-2}$ in the envelope of a Bonner-Ebert sphere (Shu 1977), the rotating models show a variety of exponents depending on $(\alpha, \beta, s)$.

The run of central angular velocity versus central density is shown for several representative sequences in figure 4.11. The dashed lines show the dependences $n \sim p^{x}, x=1 / 3,1 / 2,2 / 3$. The data are generally power-law, with $x$ lying in this range.

Further features of the eight sequences are given in figures 4.12 through 4.16. and tables 4.3 through 4.10. The system of units is based on $G, \mu$, and $\xi_{e}$ (the equatorial radius of the spheroid); and the angular velocities are measured in units of $\left(4 \pi \mathrm{G} \rho_{c}\right)^{1 / 2}$ to be consistent with the published data on rapidly rotaling polytropes (Bodenheimer and Ostriker 1973). 
Sequence A

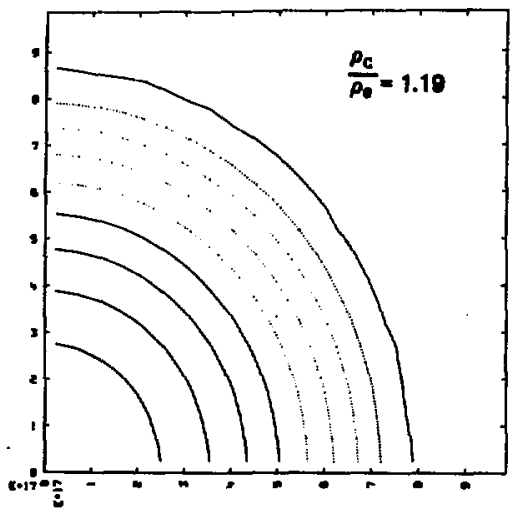

$(\alpha, \beta)=(6.49,0.033)$

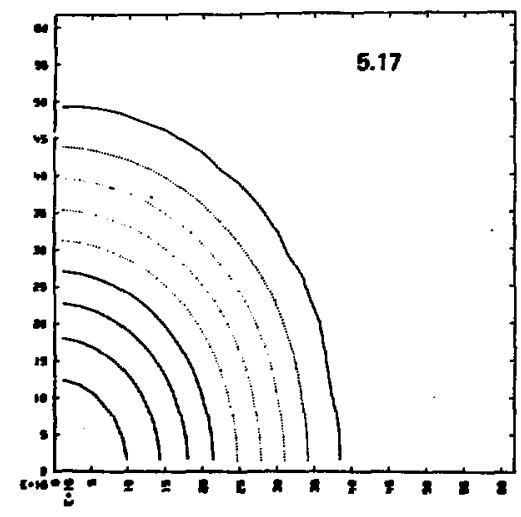

(0.91, 0.061)

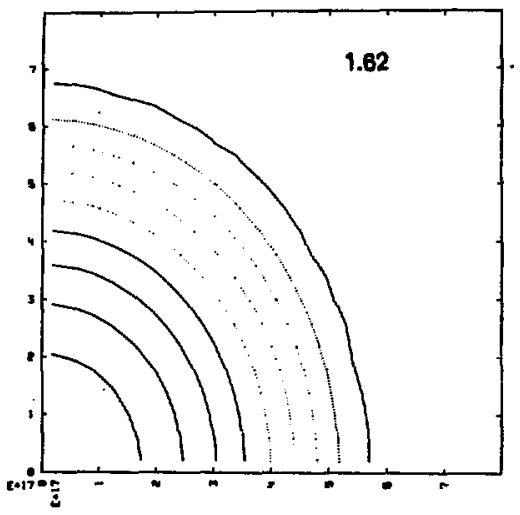

$(2.41,0.042)$

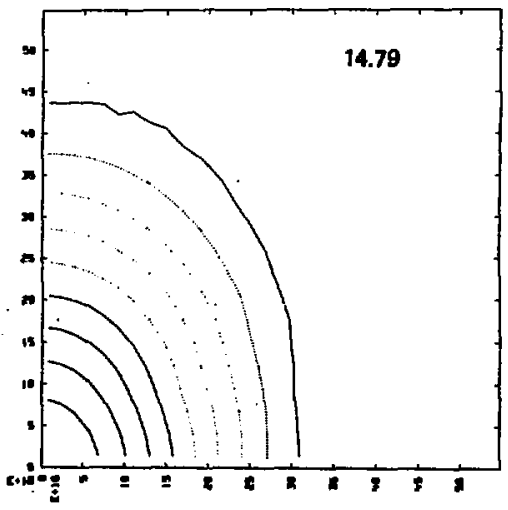

(0.61, 0.073)

Figure 4.2 
Sequence B

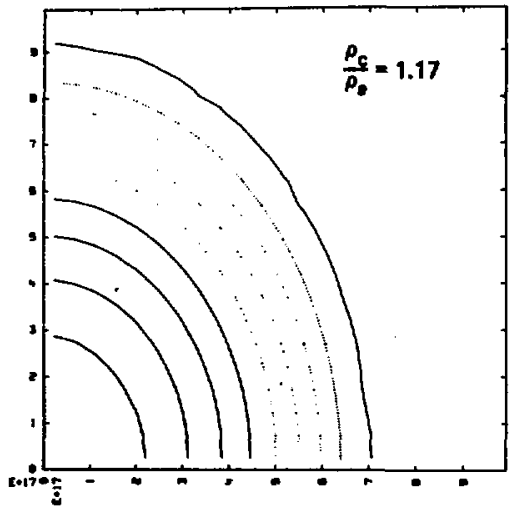

$(\alpha, \beta)=(6.55,0.038)$

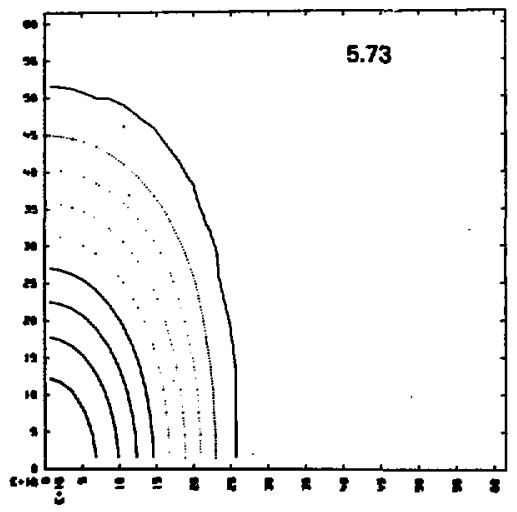

$(0.70,0.156)$

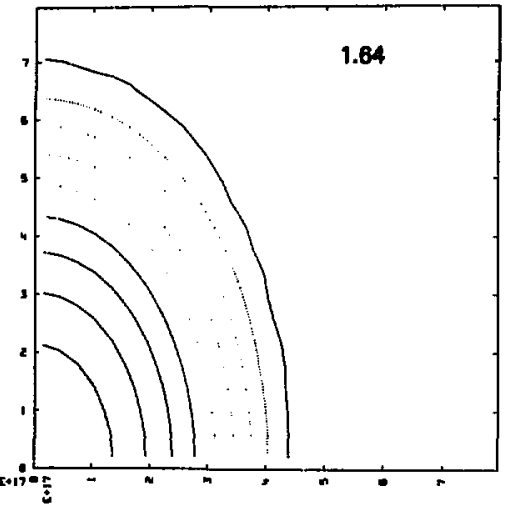

$(2.01,0.111)$

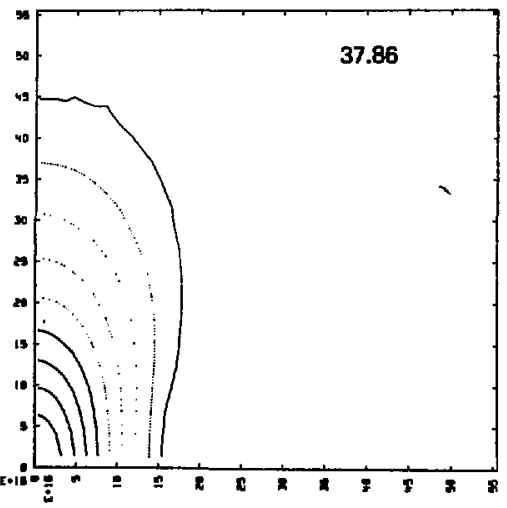

(0.44, 0.194)

Figure 4.3 


\section{Sequence $B^{\prime}$}

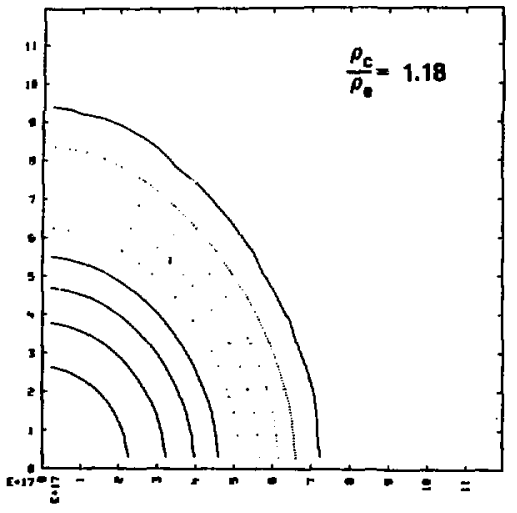

$(\alpha, \beta)=(6.56,0.091)$

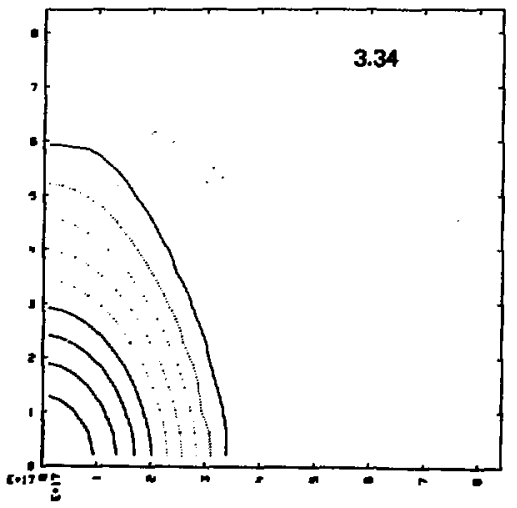

$(0.00,0.138)$

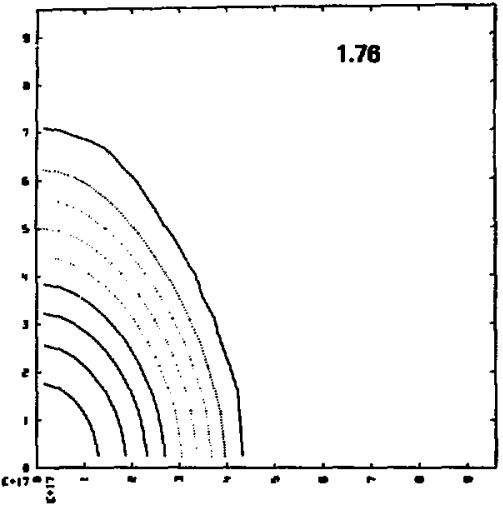

$(1.82,0.116)$

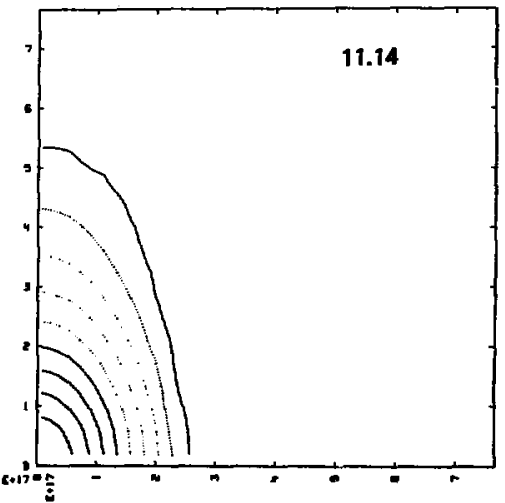

$(0.62,0.159)$

Figure 4.4 
Sequence B"

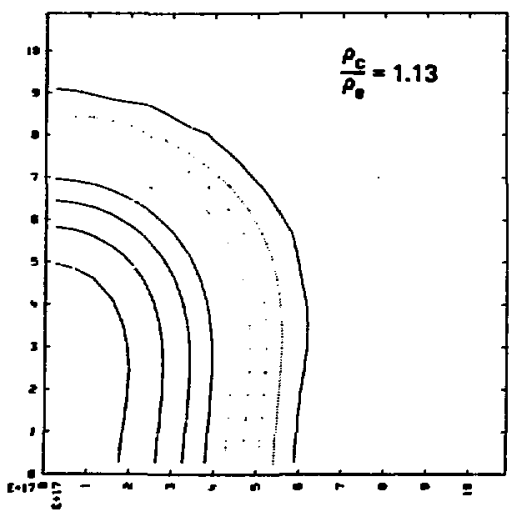

$(\alpha, \beta)=(6.59,0.87)$

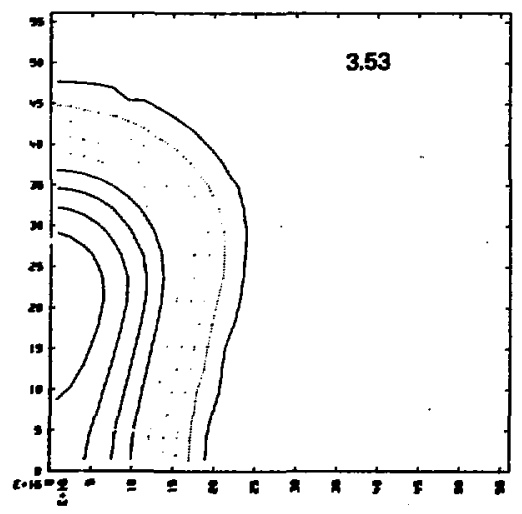

$(0.65,0.162)$

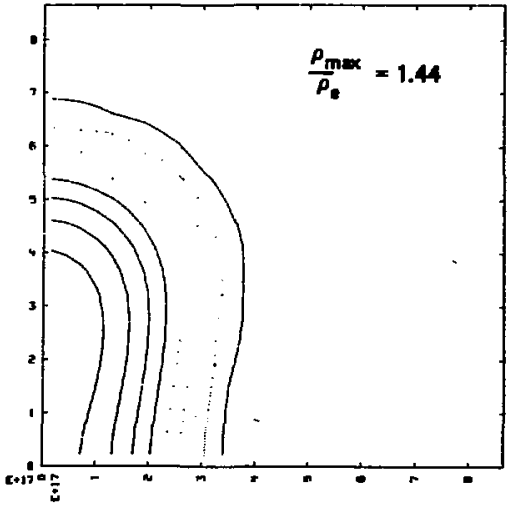

(1.96, 0.121)

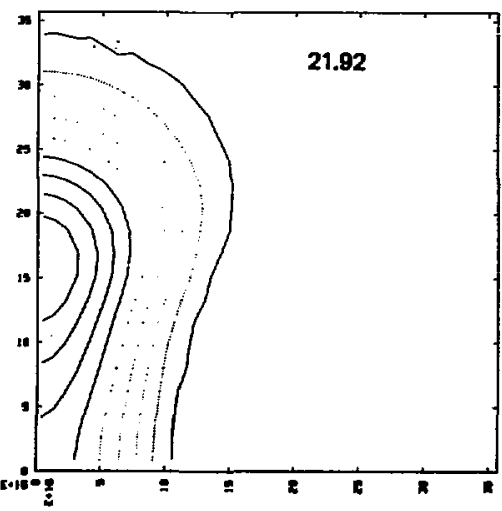

(0.31, 0.215)

Figure 4.5 
Sequence C
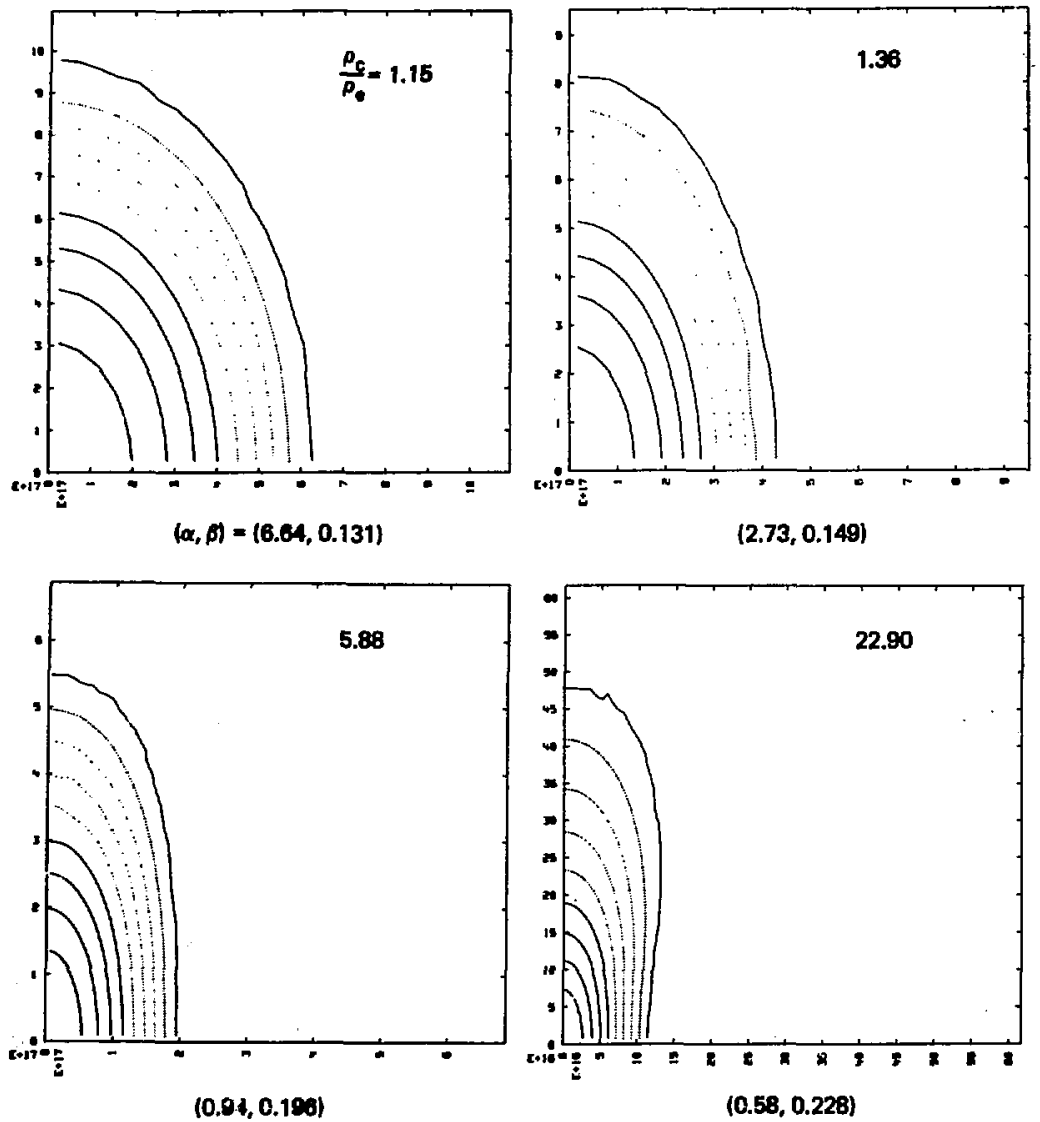

Figure 4.6 
Sequence D

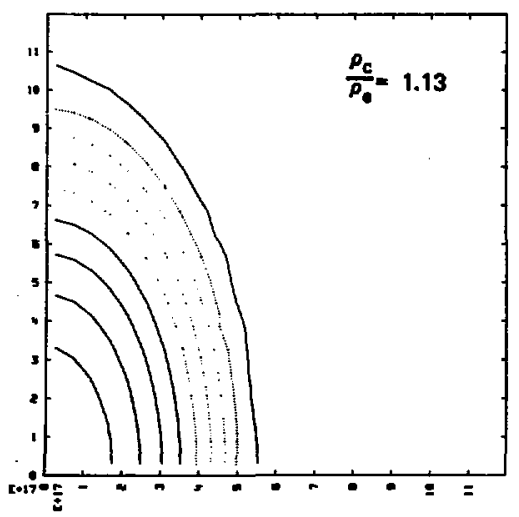

$(\alpha, \beta)=(6.80,0.160)$

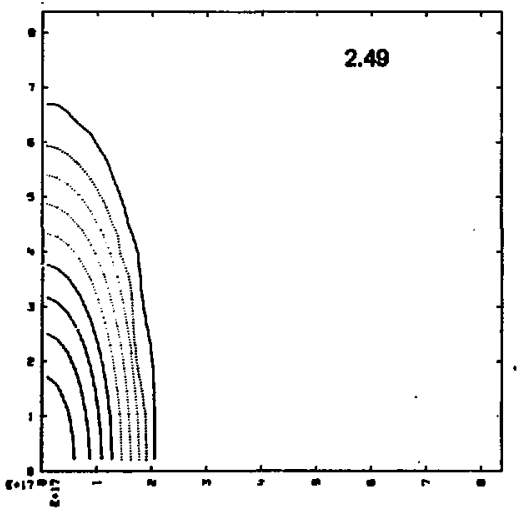

$(0.99,0.248)$

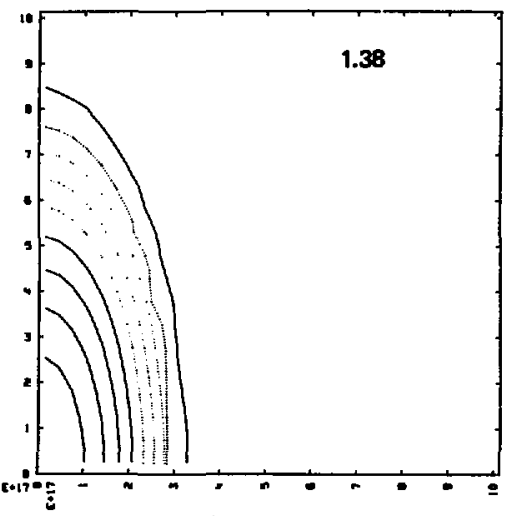

$(2.11,0.195)$

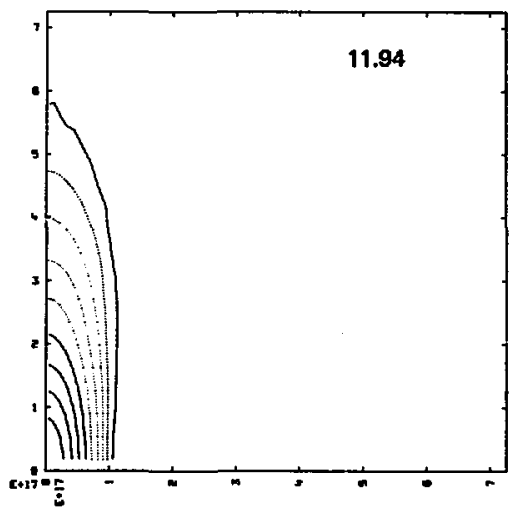

$(0.36,0.313)$

Figure 4.7 
Sequence $E$

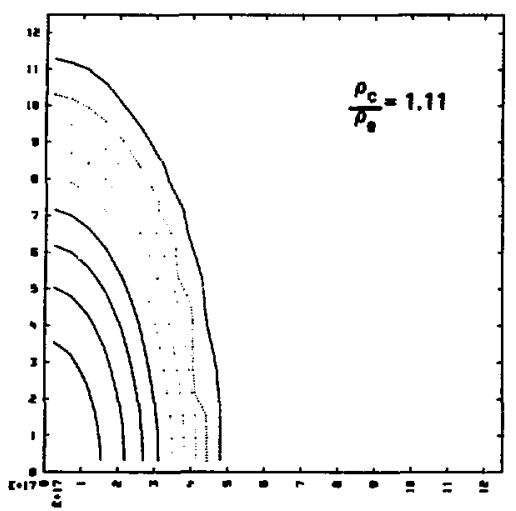

$(\alpha, \beta)=(6.99,0.210)$

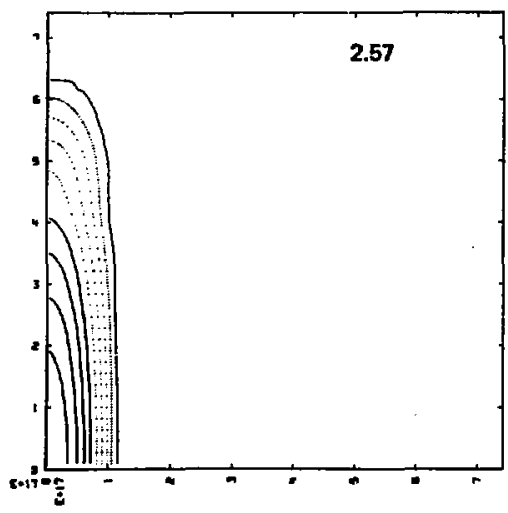

$(0.47,0.392)$

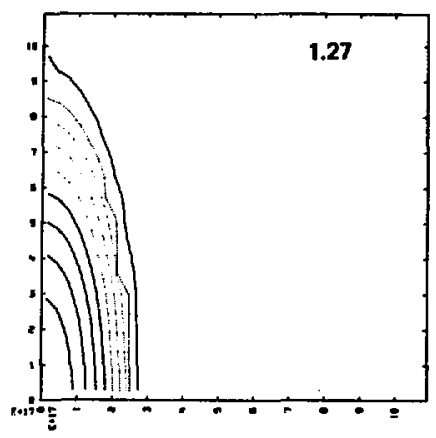

(2.35, 0.239)

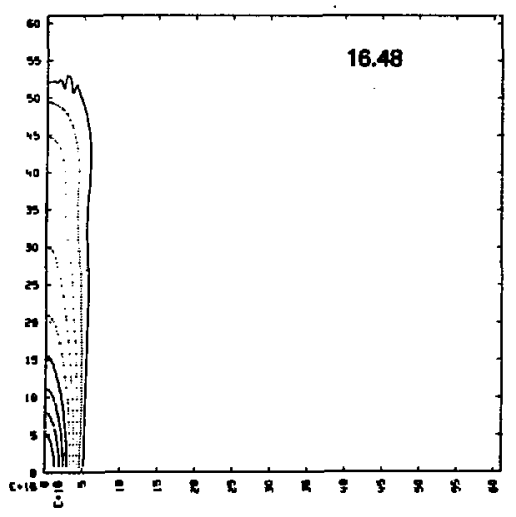

(0.26, 0.385)

Figure 4.8 
Figures 4.2-4.8--Equidensity contours of rotating, isothermal equilibria for several sequences. Rolation axis is horizontal. Lower, $r$ ght-hand model in each sequence is near criticality to collapse. Tabulated are the energy ratios. $\alpha$ and $\beta$, and the compression, $\rho_{c} / \rho_{.}$. The cloud boundary is the outer solid line. 

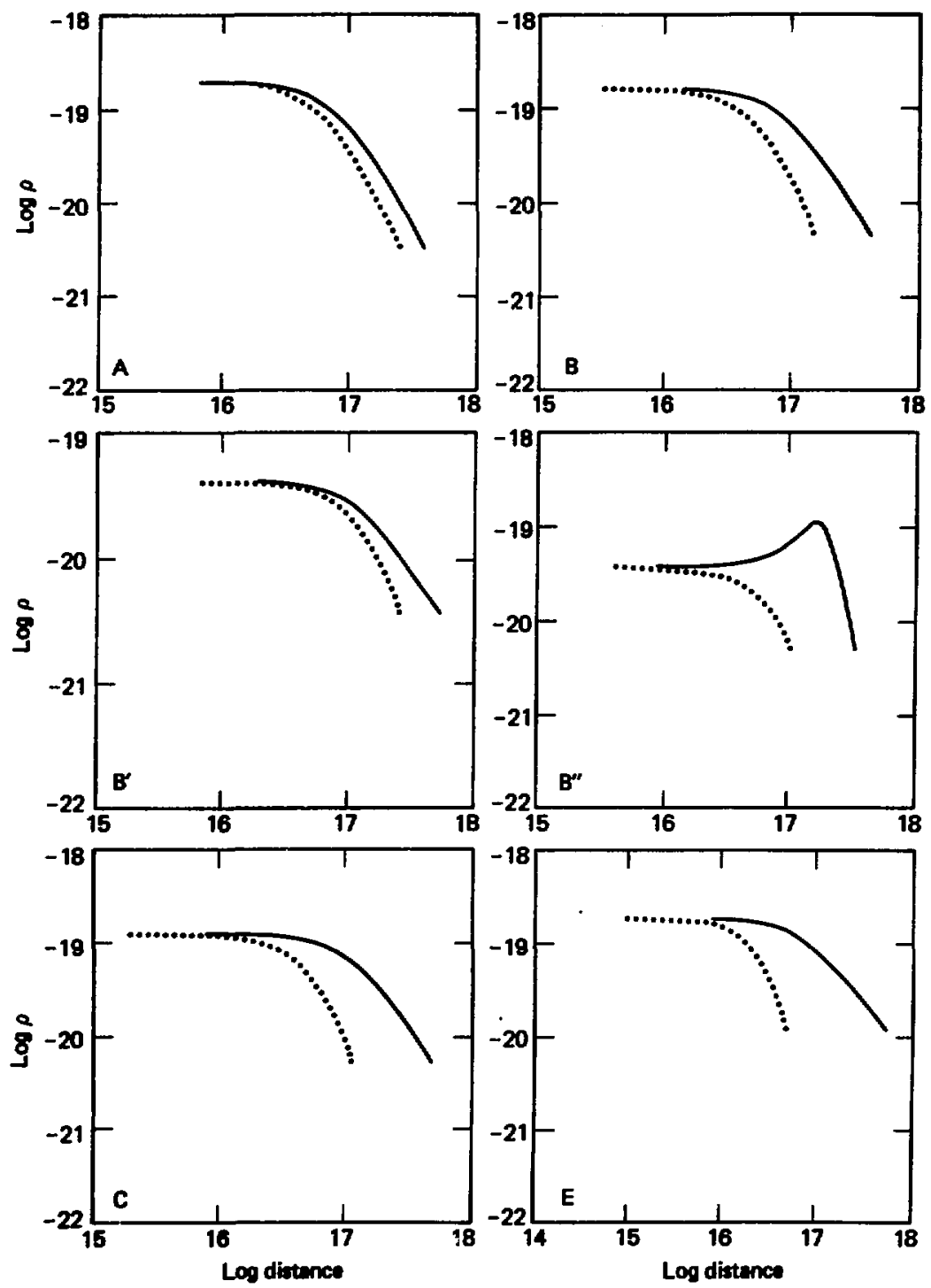

Figure 4.9 
Figure 4.9--Distributions of mas density in the equatorial plane (aolid line), and on the rotation axis (dotted line), for the critical models of sequences $4 \mathrm{~A}, 4 \mathrm{~B}^{\circ}, 4 \mathrm{~B}^{\circ}, 4 \mathrm{~B}^{\prime}, 4 \mathrm{C}$, and $4 \mathrm{E}$. 

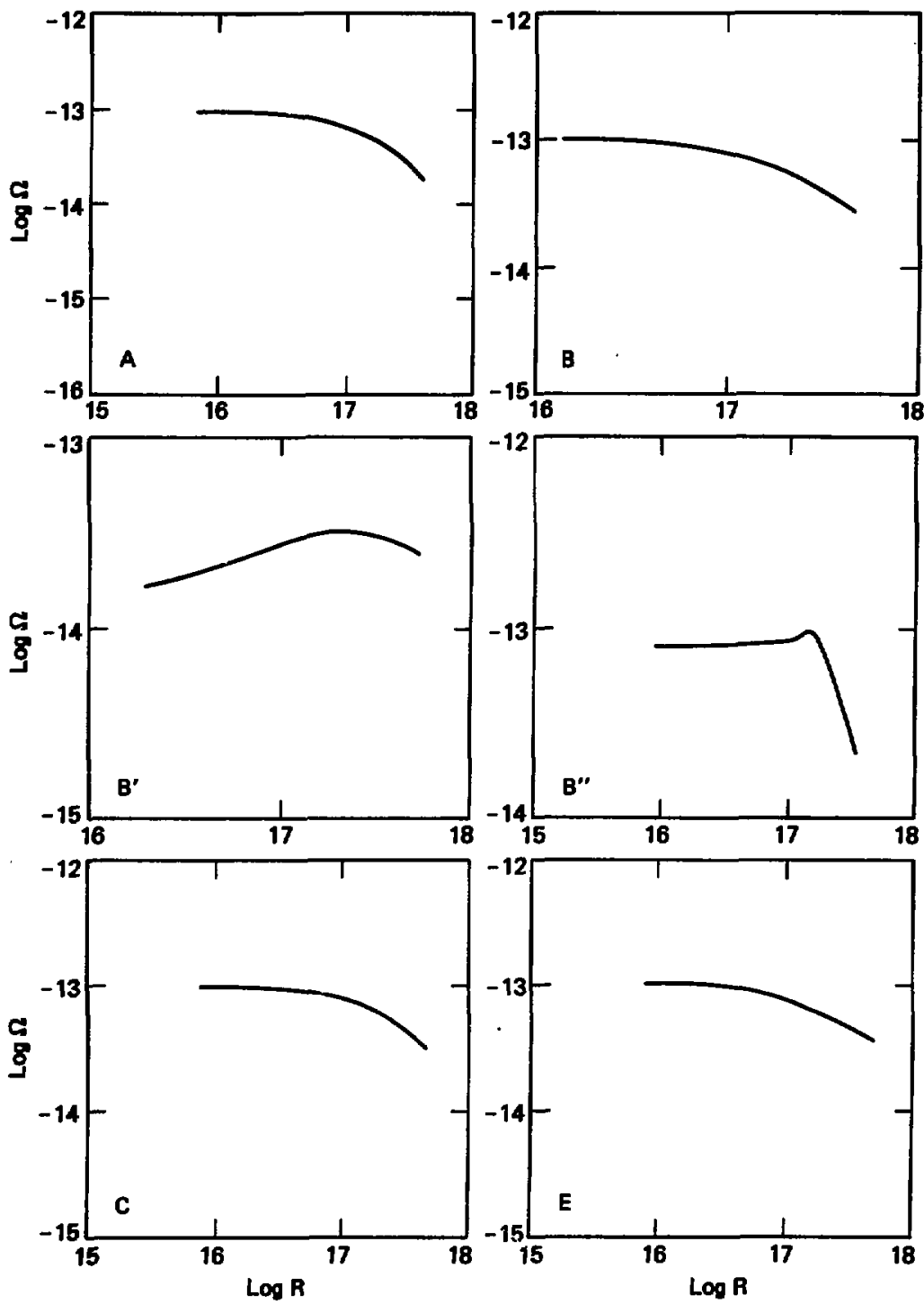

Figure 4.10

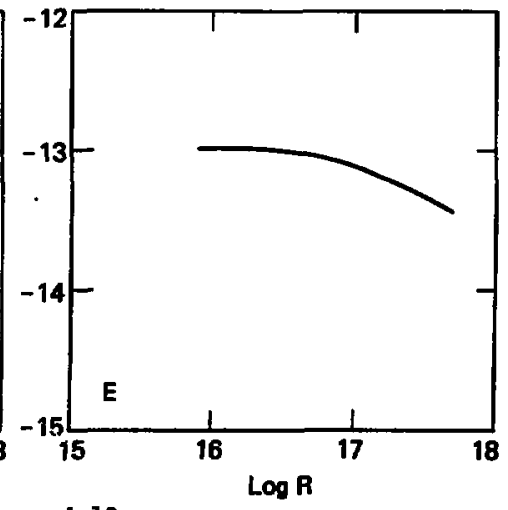


Figure 4.10-Distributions of angular velocity in the equatorial plane for the critical models of sequences $4 A, 4 B^{\prime}, 4 B^{\prime}, 4 B^{\prime}, 4 C$, and $4 E$. 


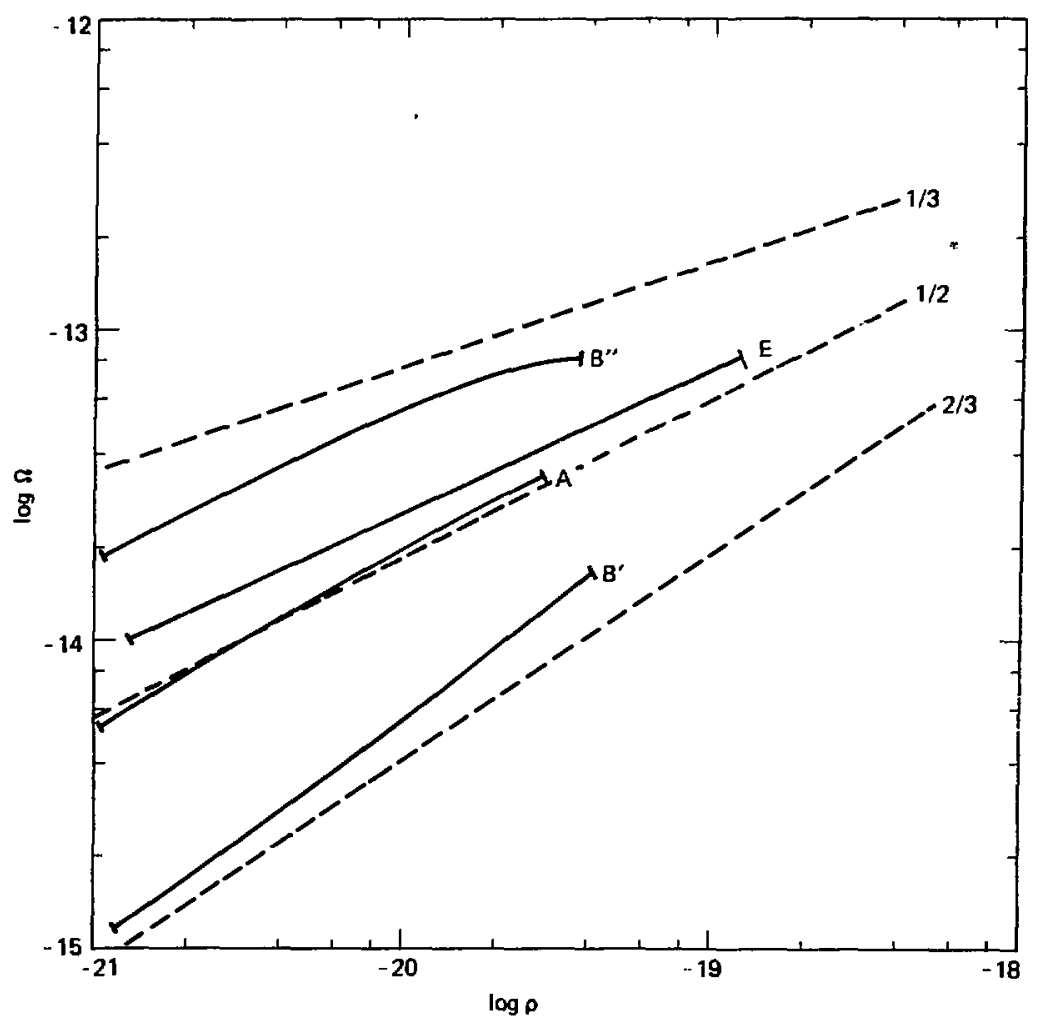

Figure 4.11-History of the central values of angular velocity, $n$, and mass density, $\rho$, for sequences $4 A, 4 B^{\prime}, 4 B^{\prime}$, and $4 E$. Power-laws of exponents $1,3,1 / 2,2 / 3$ are shown in dashed 1 ines.

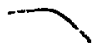


Figure 4.12 shows the ratio of equatorial to polar ridius, $\xi_{\mathbb{d}} / \xi_{p}$, as a function of $\beta$. The lower and upper dashed lines connect the initial and final states, respectively. The inset shows how the distribution of angular monentum qualitatively effects the lattening. Flattening is not a particularly relevant quantity for the ring equilibrium.

Figure 4.13 shows $\langle\Omega\rangle=J / l$ as a function of $\beta$. < $\delta>$ decreases with increasing $\beta$ for all except cascs $4 \mathrm{~A}$ and $4 \mathrm{~B} \cdots$. While the dimensional $<\Omega>$ increases along the sequences, the normalization is such that whenever the moment of inertia increases faster than the rotational energy, $<\Omega>$ decreases. Isothermal clouds are very compressible systems. The turnover of $<\Omega>$ in sequence $4 \mathrm{~A}$ is similar to the behavior of the Maclaurin spheroids and rapidly rotaling polytropes (Badenheimer and Ostriker 1973). Presumably sequences 4B, $4 \mathrm{~B}^{\circ}, 4 \mathrm{C}, 4 \mathrm{D}, 4 \mathrm{E}$ and $4 \mathrm{~F}$ were started past turnover. Curve $4 \mathrm{~B}^{\prime \prime}$ shows quite a different behavior from the rest. The dot-dashed line in figure 4.13 uses $\left(4 \pi \mathrm{G} \rho_{\max }\right)^{1 / 2}$ as the normalizing constant.

Figure 4.14 shows the degree of differential rotation as a function of $\beta$ for the eight sequences. It is interesting that $\Omega_{e} / \Omega_{c}$ decreases for the initially differentially rotating cloud. As the ring grows, apparently very little differential rotation is needed to support it.

Figures 4.15 and 4.16 show the degree of compression as a function of $\eta$. For a given $\eta$ the more rapidly rotating spheroids need less compression for support; the support comes from rotation. It is interesting that the compression at the critical states is not a monotonic function of angular momentum. The reason for this is not known. The dotted line is the sequence of Bonner-Ebert spheres, which became critical at $\rho_{c} / \rho_{e}=15.4$. This number is 8 percent larger than the actual value of 14.3 for the critical Bonner-Ebert 


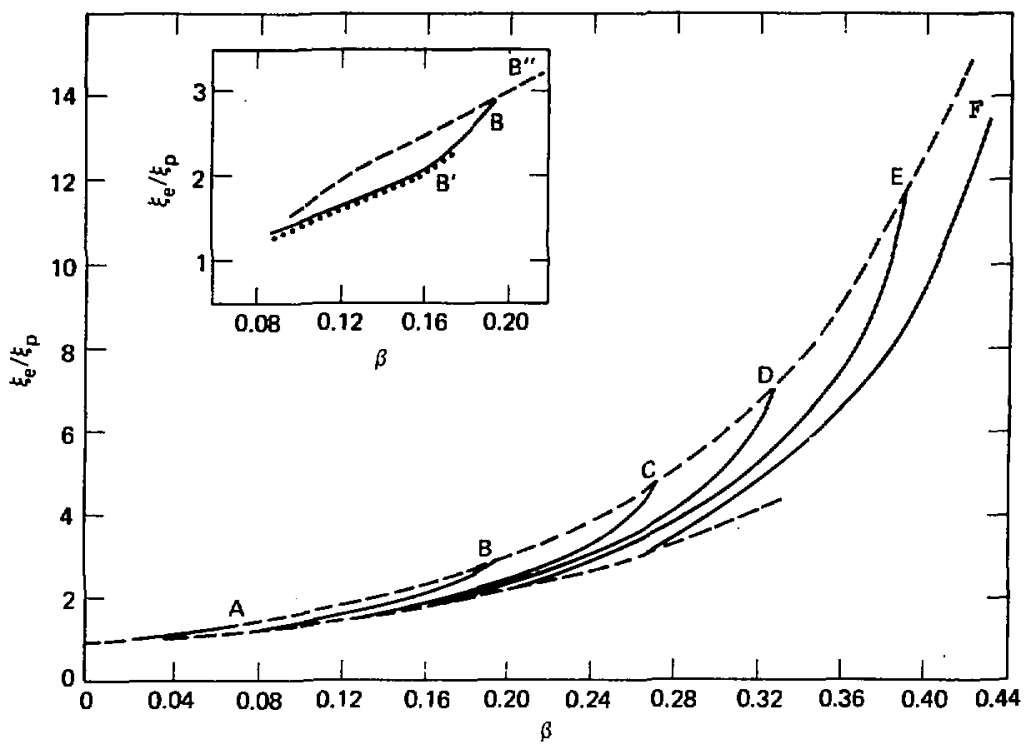

Figure 4.12-Flattening, $\xi_{e} / \xi_{p}$, for eight cooling sequences as a function of the rotation parameter. $\beta$. Initial states and final states are connected by dashed $I$ ines. 


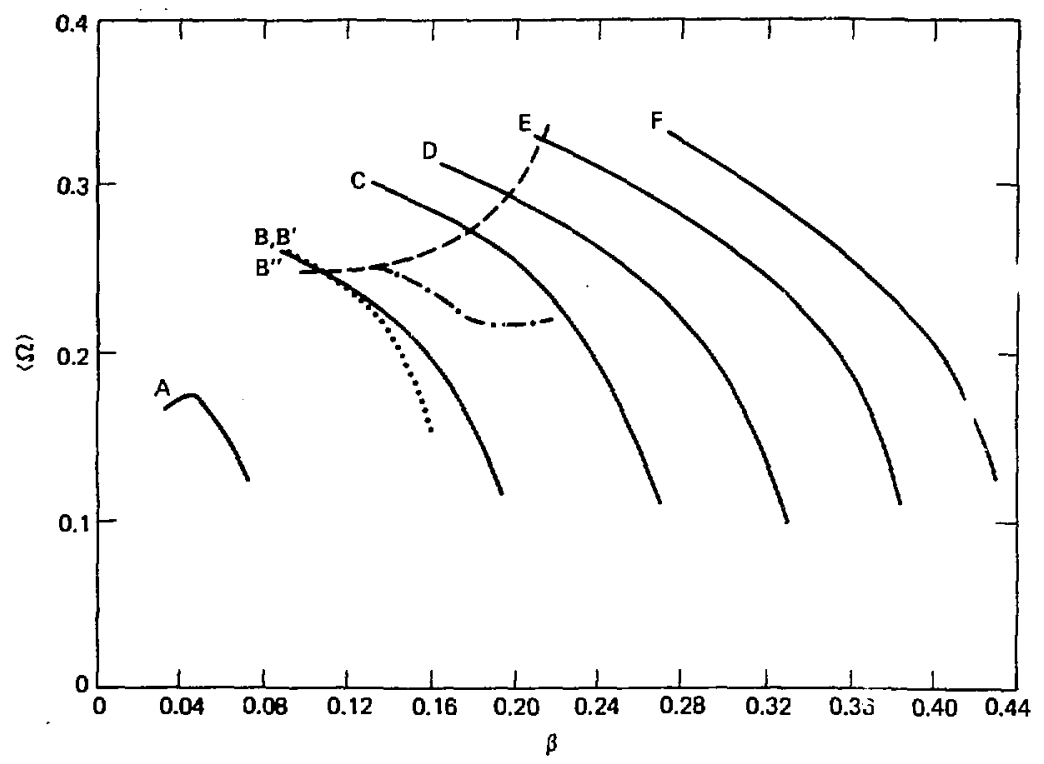

Figure 4.13--Average angular velocity, $4 \Omega>$, measured in inits of $\left(4 \pi G \rho_{c}\right)^{1 / 2}$. for eight cooling sequences as a function of $\beta$. The dot-dashed curve is normalized by $\left(4 \pi \mathrm{G} \rho_{\text {max }}\right)^{1 / 2} ; \rho_{\text {max }}$ is the maximum $r i n g$ density. 


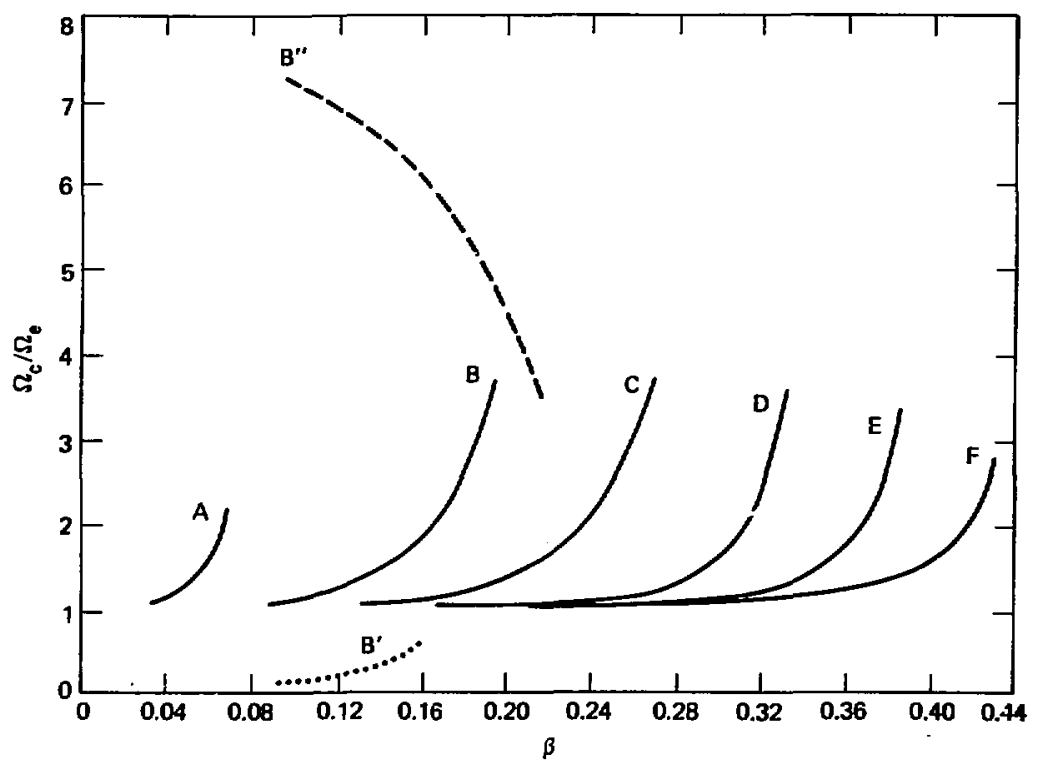

in

Figure 4.14-Degree of differential rotation, $\Omega_{c} / \Omega_{*}$, for eight cooling sequences as function of $\beta$. 


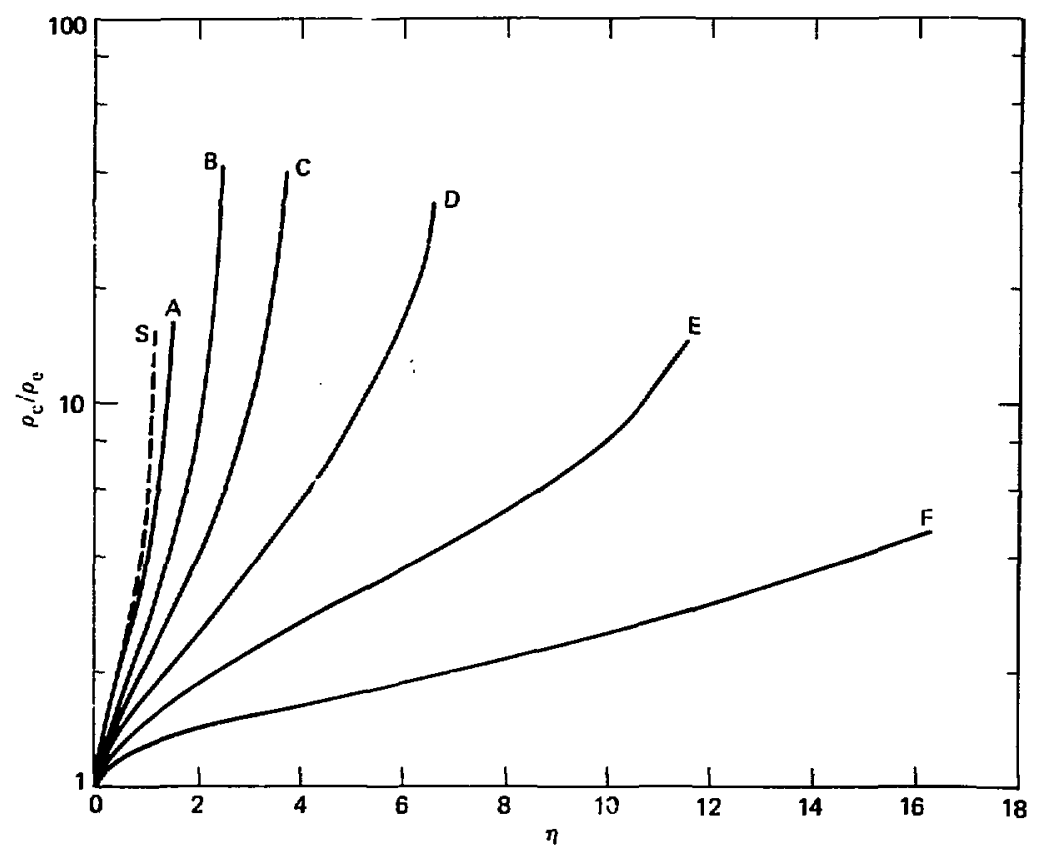

Figure 4.15 
74

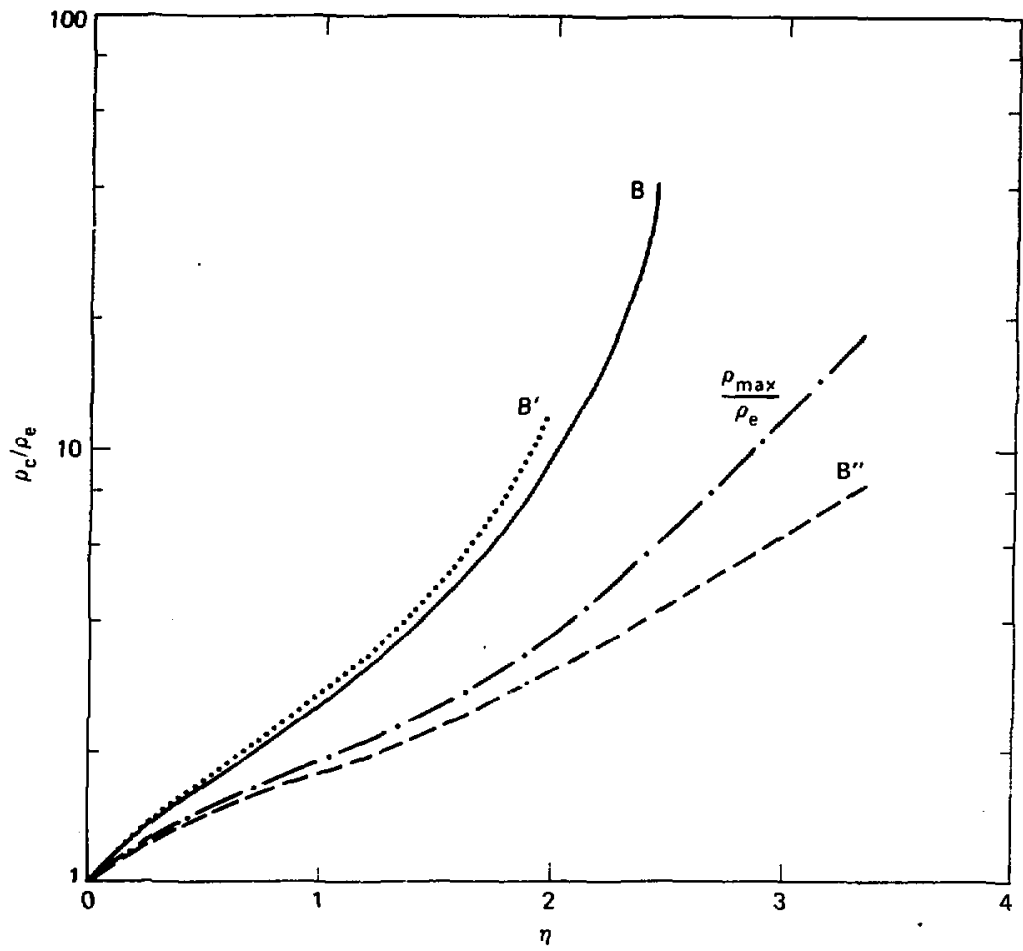

Figure 4.16 
Figures 4.15 and 4.16 -Compression, $\rho_{c} / \rho_{e}$, for eight cooling sequences as a function of critical masses, $\eta$. 
sphere, and therefore 8 percent may be taken for a rough error estimate for the olher numbers.

Additional quantities listed in tables 4.3 to 4.10 are normalized angular momentum, energy, moment of inertia, and central density and angular velocity. 
TABLE 4.3

PHYSICAL PROPERITIES OF SEQUENCE A

\begin{tabular}{cccccccc}
$\mathrm{J}$ & $\mathrm{K}$ & $\mid \mathrm{WI}$ & $\mathrm{U}_{\mathrm{T}}$ & $\mathrm{I}_{11}$ & $\rho_{c}$ & $\Omega_{c}$ & $\xi_{c} / \xi_{\mathrm{p}}$ \\
\hdashline & - & - & - & - & - & - & -1.10 \\
0.127 & 0.020 & 0.623 & 6.736 & 0.396 & 0.292 & 0.178 & 1.10 \\
0.143 & 0.027 & $0.65 \mathrm{l}$ & 2.620 & 0.377 & 0.385 & 0.192 & 1.19 \\
0.168 & 0.044 & 0.720 & 1.089 & 0.329 & 0.877 & 0.215 & 1.29 \\
0.178 & 0.058 & 0.793 & 0.666 & 0.292 & 1.984 & 0.228 & 1.41 \\
\hline
\end{tabular}

TABLE 4.4

PHYSICAL PROPERITIES OF SEQUENCE B

\begin{tabular}{cccccccc}
$J$ & $K$ & $|W|$ & $U_{\mathrm{T}}$ & $I_{11}$ & $\rho_{\mathrm{c}}$ & $\Omega_{\mathrm{c}}$ & $\xi_{\mathrm{e}} / \xi_{\mathrm{p}}$ \\
& - & - & - & - & - & - & - \\
0.213 & 0.057 & 0.655 & 7.155 & 0.395 & 0.342 & 0.269 & 1.31 \\
0.244 & 0.078 & 0.703 & 2.357 & 0.381 & 0.509 & 0.275 & 1.59 \\
0.285 & 0.124 & 0.795 & 0.920 & 0.333 & 1.391 & 0.283 & 2.00 \\
0.298 & 0.155 & 0.871 & 0.753 & 0.301 & 3.109 & 0.282 & 2.39 \\
0.305 & 0.184 & 0.947 & 0.690 & 0.278 & 7.180 & 0.280 & 2.90 \\
\hline
\end{tabular}


TABLE 4.5

PHYSICAL PROPERITIES OF SEQUENCE B'

\begin{tabular}{|c|c|c|c|c|c|c|c|}
\hline$J$ & $\mathbf{K}$ & $|W|$ & $\mathrm{U}_{\mathrm{T}}$ & $I_{11}$ & $p_{c}$ & $\Omega_{c}$ & $\xi_{\mathbb{e}} / \xi_{\mathrm{p}}$ \\
\hline 0.213 & 0.060 & 0.655 & 7.270 & 0.385 & 0.383 & 0.037 & 1.29 \\
\hline 0.245 & 0.084 & 0.725 & 2.183 & 0.368 & 0.603 & 0.046 & 1.62 \\
\hline 0.267 & 0.106 & 0.770 & 1.273 & 0.343 & 1.019 & 0.061 & 1.76 \\
\hline 0.281 & 0.139 & 0.875 & 0.912 & 0.295 & 3.100 & 0.090 & 2.08 \\
\hline
\end{tabular}

TABLE 4.6

PHYS ICAL PROPERITIES OF SEQUENCE B"

\begin{tabular}{ccccccccc}
$\mathrm{J}$ & $\mathrm{K}$ & $|\mathrm{W}|$ & $\mathrm{U}_{\mathrm{T}}$ & $\mathrm{I}_{1 \mathrm{~L}}$ & $\rho_{\mathrm{c}}$ & $\Omega_{\mathrm{c}}$ & $\xi_{\mathrm{e}} / \xi_{\mathrm{p}}$ \\
\hline & - & - & - & - & - & - & - \\
0.207 & 0.062 & 0.642 & 7.050 & 0.418 & 0.315 & 0.660 & 1.53 \\
0.239 & 0.083 & 0.683 & 2.224 & 0.415 & 0.423 & 0.626 & 2.01 \\
0.286 & 0.119 & 0.736 & 0.783 & 0.387 & 0.605 & 0.607 & 2.49 \\
0.339 & 0.185 & 0.862 & 0.444 & 0.328 & 0.725 & 0.455 & 3.19 \\
\hline
\end{tabular}


TABLE 4.7

\section{PHYSICAL PROPERITIES OF SEQUENCE C}

\begin{tabular}{cccccccc}
$\mathrm{J}$ & $\mathrm{K}$ & $|\mathrm{W}|$ & $\mathrm{U}_{\mathrm{T}}$ & $\mathrm{I}_{11}$ & $\rho_{\mathrm{c}}$ & $\Omega_{\mathrm{c}}$ & $\xi_{\mathrm{e}} / \xi_{\mathrm{p}}$ \\
\hline & - & - & - & - & - & - & - \\
0.266 & 0.090 & 0.686 & 7.604 & 0.393 & 0.403 & 0.307 & 1.55 \\
0.291 & 0.109 & 0.727 & 3.300 & 0.389 & 0.547 & 0.310 & 1.91 \\
0.334 & 0.153 & 0.782 & 1.223 & 0.365 & 0.985 & 0.309 & 2.32 \\
0.356 & 0.194 & 0.851 & 0.814 & 0.332 & 1.997 & 0.304 & 2.86 \\
0.370 & 0.254 & 0.974 & 0.641 & 0.285 & 7.353 & 0.294 & 4.09
\end{tabular}

TABLE 4.8

PHYS ICAL PROPERITIES OF SEQUENCED

\begin{tabular}{cccccccc}
$\mathrm{J}$ & $\mathrm{K}$ & $|\mathrm{W}|$ & $\mathrm{U}_{\mathrm{T}}$ & $\mathrm{I}_{11}$ & $\rho_{\mathrm{q}}$ & $0_{\mathrm{c}}$ & $\xi_{\mathrm{e}} / \xi_{\mathrm{P}}$ \\
- & - & - & - & - & - & - & - \\
0.304 & 0.119 & 0.728 & 0.255 & 0.388 & 0.506 & 0.316 & 1.93 \\
0.340 & 0.152 & 0.778 & 2.740 & 0.384 & 0.759 & 0.310 & 2.59 \\
0.382 & 0.207 & 0.835 & 1.112 & 0.359 & 1.370 & 0.303 & 3.05 \\
0.411 & 0.307 & 0.984 & 0.588 & 0.298 & 6.808 & 0.282 & 5.50 \\
0.416 & 0.343 & 1.041 & 0.527 & 0.284 & 18.520 & 0.274 & 7.19 \\
\hline
\end{tabular}


TABLE 4.9

PHYSICAL PROPERITIES OF SEQUENCE E

\begin{tabular}{cccccccc}
$\mathrm{J}$ & $\mathrm{K}$ & $\mid \mathrm{WI}$ & $\mathrm{U}_{\mathrm{T}}$ & $\mathrm{I}_{11}$ & $\rho_{\mathrm{c}}$ & $\Omega_{\mathrm{c}}$ & $\xi_{\mathrm{c}} / \xi_{\mathrm{p}}$ \\
\hline & - & - & - & - & - & - & - \\
0.352 & 0.159 & 0.757 & 8.823 & 0.390 & 0.606 & 0.329 & 2.36 \\
0.381 & 0.198 & 0.829 & 3.243 & 0.371 & 1.011 & 0.301 & 3.49 \\
0.421 & 0.255 & 0.869 & 1.355 & 0.358 & 1.570 & 0.296 & 4.26 \\
0.442 & 0.327 & 0.955 & 0.745 & 0.320 & 3.390 & 0.272 & 6.18 \\
0.447 & 0.443 & 1.135 & 0.479 & 0.260 & 35.00 & 0.260 & 11.67 \\
- & & & & & & & \\
\hline
\end{tabular}

TABLE 4.10

PHYSICAL PROPERITIES OF SEQUENCE F

\begin{tabular}{ccccccccc}
$J$ & $K$ & $|W|$ & $U_{\mathrm{T}}$ & $I_{11}$ & $\rho_{\mathrm{c}}$ & $\Omega_{\mathrm{c}}$ & $\xi_{\mathrm{c}} / \xi_{\mathrm{p}}$ \\
- & - & - & - & - & - & - & - \\
0.408 & 0.220 & 0.808 & 9.938 & 0.379 & 0.844 & 0.335 & 3.17 \\
0.435 & 0.255 & 0.863 & 3.678 & 0.381 & 1.466 & 0.279 & 5.21 \\
0.475 & 0.317 & 0.897 & 1.495 & 0.371 & 2.191 & 0.260 & 6.96 \\
0.496 & 0.379 & 0.951 & 0.799 & 0.349 & 4.058 & 0.254 & 9.40 \\
0.502 & 0.440 & 1.024 & 0.500 & 0.322 & 13.450 & 0.250 & 13.28 \\
\hline
\end{tabular}


4.5) Stability and Collapse

Discussions of the equilibrium spherolds (Maclaurin, polytropic) usually consider their secular and dynamic instability to triaxial and ring modes (Tassoul 1978, Ostriker and Bodenheimes 1973). A third global mode of instability is possible for the isothermal spheroids: gravitational collapse. Since the existence of triaxial equilibria for isothermal clouds has not been established, we discuss the second and third types of instability.

4.5.1) Stability

A direct test of the stability of a disk equilibrium has been made to see if a ring mode can be excited for high $\beta(\geqslant 0.36)$. The procedure was to repeat the high $\beta$ segment of cooling sequence $4 \mathrm{E}$, applying the constant pressure boundary condition with intercloud density equal to the cloud density at the surface. Thus the boundary was neutrally stable and large perturbations resulted as waves propagated along its length. Figure 4.17 shows several meridional sections before and after the appearance of the ring. Albejt a crude determination, $0.37 \leqslant \beta_{c r i t} \leqslant 0.40$. This number is consistent with the work of Bardeen (1971), who calculated $\beta=0.359$ and $\beta=0.457$ for the onset of secular and dynamic axisymetric instabilities in Maclaurin spheraids.

\subsection{2) Collapse}

The termination of the cooling sequences $4 \mathrm{~A}$ to $4 \mathrm{~F}$ is shown in more detail in Iigure 4.18. Sequence $4 \mathrm{~F}$ was not run to a clear transition due to extreme flattening. Two proposed lines of criticality are included for reference: one due to Larson (1972), and the other from ajrial theorem analysis of uniform spheres. Larsona's line of criticality is based on a heuristic argument, but 


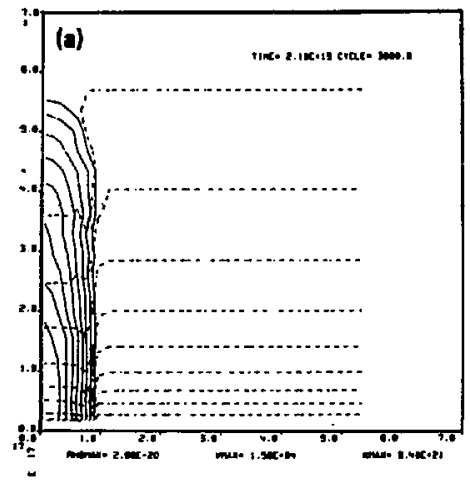

$$
(\alpha, \beta)=(0.60,0.37)
$$

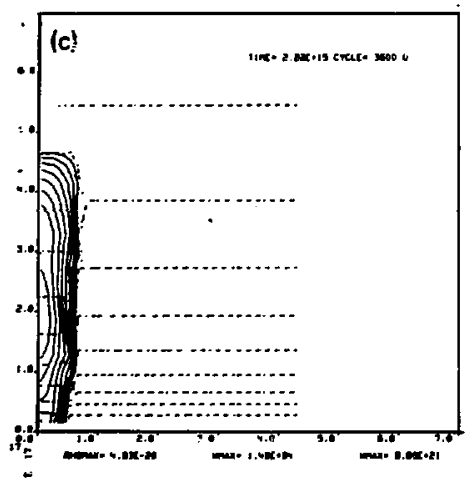

$(0.37,0.41)$

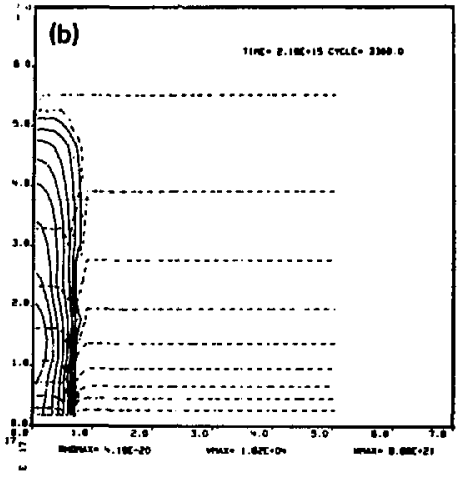

$(0.47,0.39)$

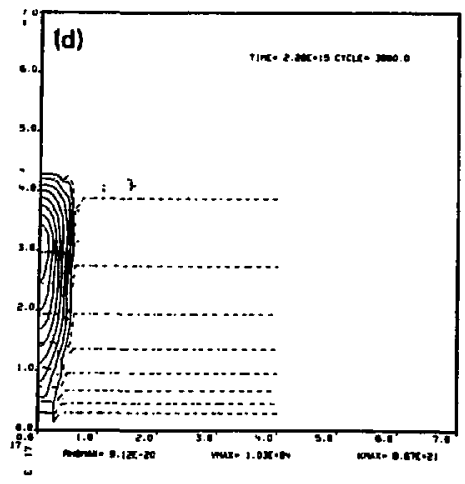

$(0.30,0.42)$

Figure 4.17--Growth of a ring instability in sequence 4E at high $\beta$, when strongly periurbed. 


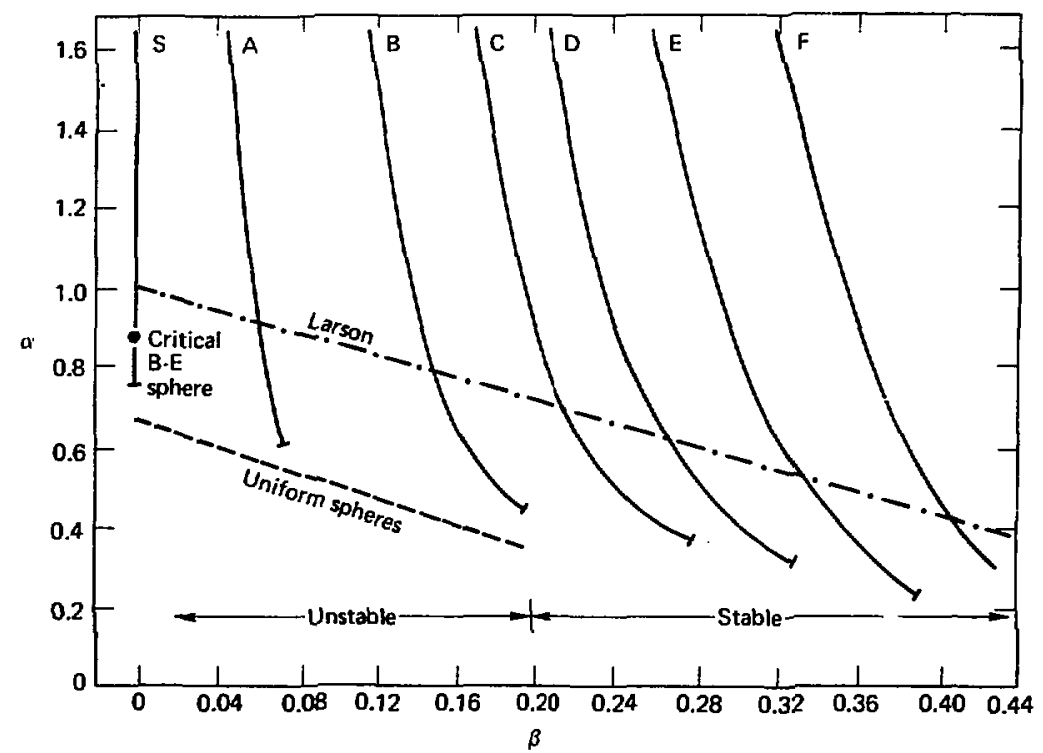

Figure 4.18--Enlargement of $\mathrm{fig}$. 4.1, showing critical points for s=1 spheroids in $(\alpha, \beta)$ space. Approximate aritical line due to Larson (1972). is based on heuristic argument (dot-cashed line). Critical line from a virial theorem analysis of uniform spheres predicts stability above $\beta=i .1849$ by rotation (dashed line). 
appears at least qualitatively correct. The virial theorem analysis predicts a $\beta=0.1949$ cutoft above which centrifugal torce stabilizes collapse. Although the flattening of the equilibrium spheroids makes an analys is based on uniform spheres inappropriate in detail, the unitorm sphere analysis bears repeating.

Applying the virial theorem to a sphere of radius $R$, we have

$$
4 \pi R^{3} P_{x}=3 \mu^{2}+\frac{1}{2 \mu R^{2}}-\frac{3 G u^{2}}{5 R} .
$$

The spiere is crilical when $\mathrm{dP}_{x} / d R=0$, therefore denoling the critical values of radius and pressure as $R_{c}$ and $P_{c}$, respectively, we have

$$
12 \pi \mathrm{R}_{c}^{2} \mathrm{P}_{c}=-\frac{5 J^{2}}{\mu \mathrm{R}_{c}{ }^{3}}+\frac{3 G \mu^{2}}{5 \mathrm{R}_{c}^{2}} .
$$

Eliminating $P_{c}$ from equation $(14)$, we can reduce (15) to a quadratic equation for $R_{c}$ :

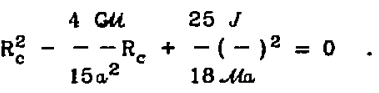

In the absense of rotation

$$
R_{0}=R_{c}(J=0)=\frac{4 c u}{15 a^{2}} .
$$

The coefficient from an analysis of Bonner-Ebert spheres is 0.41 .

Defining a volume, $v$, measured in units of $4 \pi R_{0} 3 / 3$, and a pressure, $p$. measured in units of

$$
\frac{1}{20 \pi} \frac{\mathrm{Gu}^{2}}{\mathrm{R}_{0}{ }^{4}} .
$$

then equation (14) can be transformed into the following equation of state: 


$$
p v=4+\frac{375}{8}<\frac{1}{v^{2 / 3}}-\frac{3}{v^{1 / 3}},
$$

where

$$
\zeta=\frac{\mathrm{J} a}{\mathrm{G} \boldsymbol{u}^{2}}
$$

the rotation parameter. ${ }^{2}$

Equation (18) is plotted in igure 4.19 for several values of $\xi$. The two solutions of equation (16) form volumes, call them $v_{+}$and $v_{-}$, at which $\mathrm{d} p / \mathrm{d} v=$ D. As a pressure supported sphere is compressed $i t$ moves to the left along a line of constant $\zeta$. When its volume reaches $v_{+}$it collapses to volume $v_{-}$, where it is supported by rotation. For $\zeta=0.1131, v_{+}=v_{-}$, and the unstable region has shrunk to a point. For $\zeta>0.1131$, no collapse is possible.

The rotating equilibria may behave in the same way, however with a cutof a higher $\beta$. The cooling trajectories have been plotted in figure 4.20 using the parameters $(\eta, x)$ this time. The dashed line is the critical line, above which the cloud collapses. The collapse region would be seperated from the hypothetical centrifugally supported region by cooling sequence that comes in tarifent to the critical line. The $s=1$ spheroids do not appear to have such a division for $\beta<0.50$, the virial limit. The shape of the critical line is not known for $s \neq 1$ because of the small sample of sequences calculated. Further analysis of $s \neq 1$ criticality is warranted.

2 ; $\mathrm{i}$ another form for $x=d_{g} \mathrm{P}_{x} / a^{7}$, appropriate for compression sequences. 


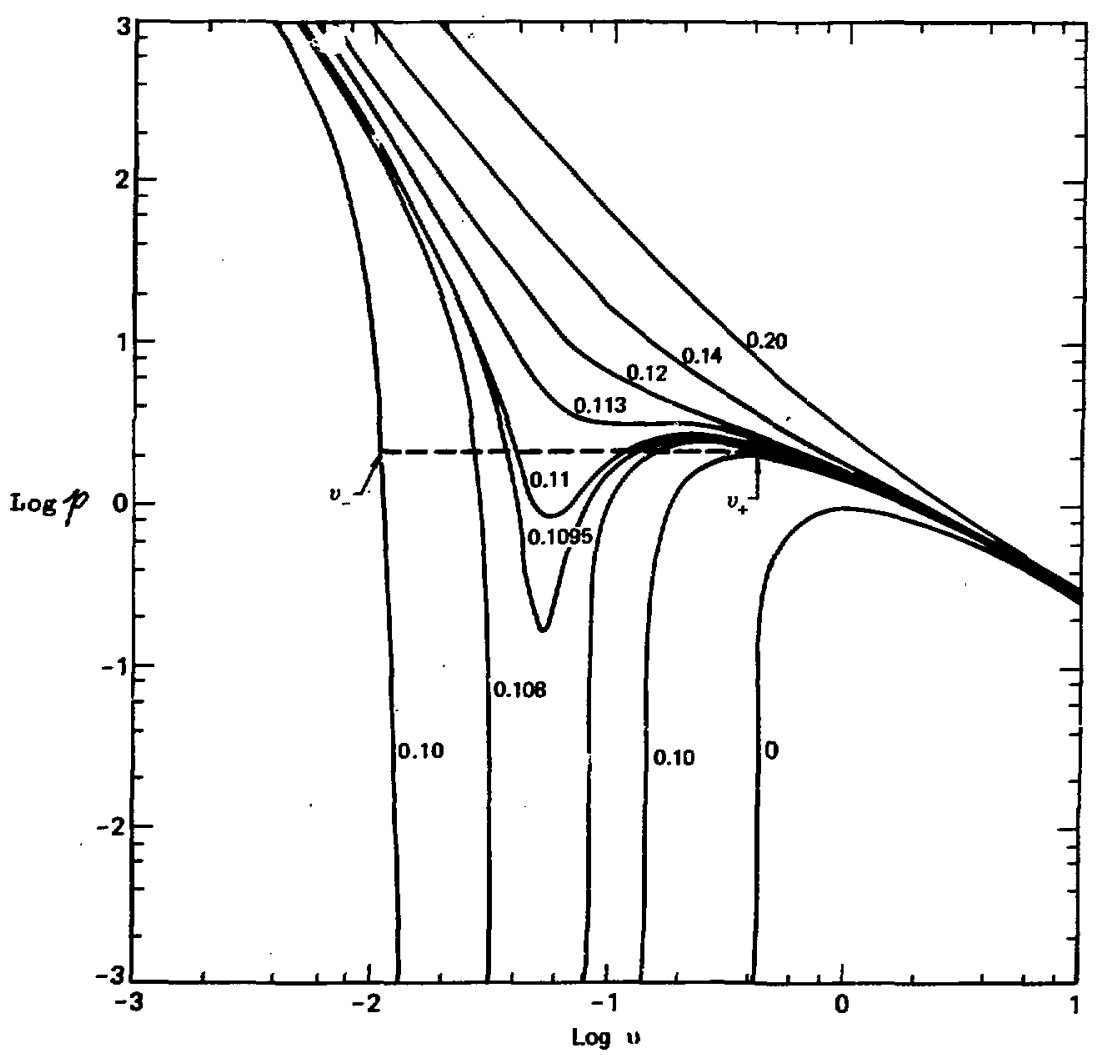

Figure 4.19--Boyle's law for a self-gravitating, rigidly rotating, uniform density gas sphere for a variety of retation parameters, $\zeta$. Repulsion at larce and sall volume, $v$, is due to pressure and centrifugal force. respectively, while attraction at intermediate volume is gravitational. Compresoing a shere leads to collapse when and if $\mathrm{dp} / \mathrm{d} v>0$. 


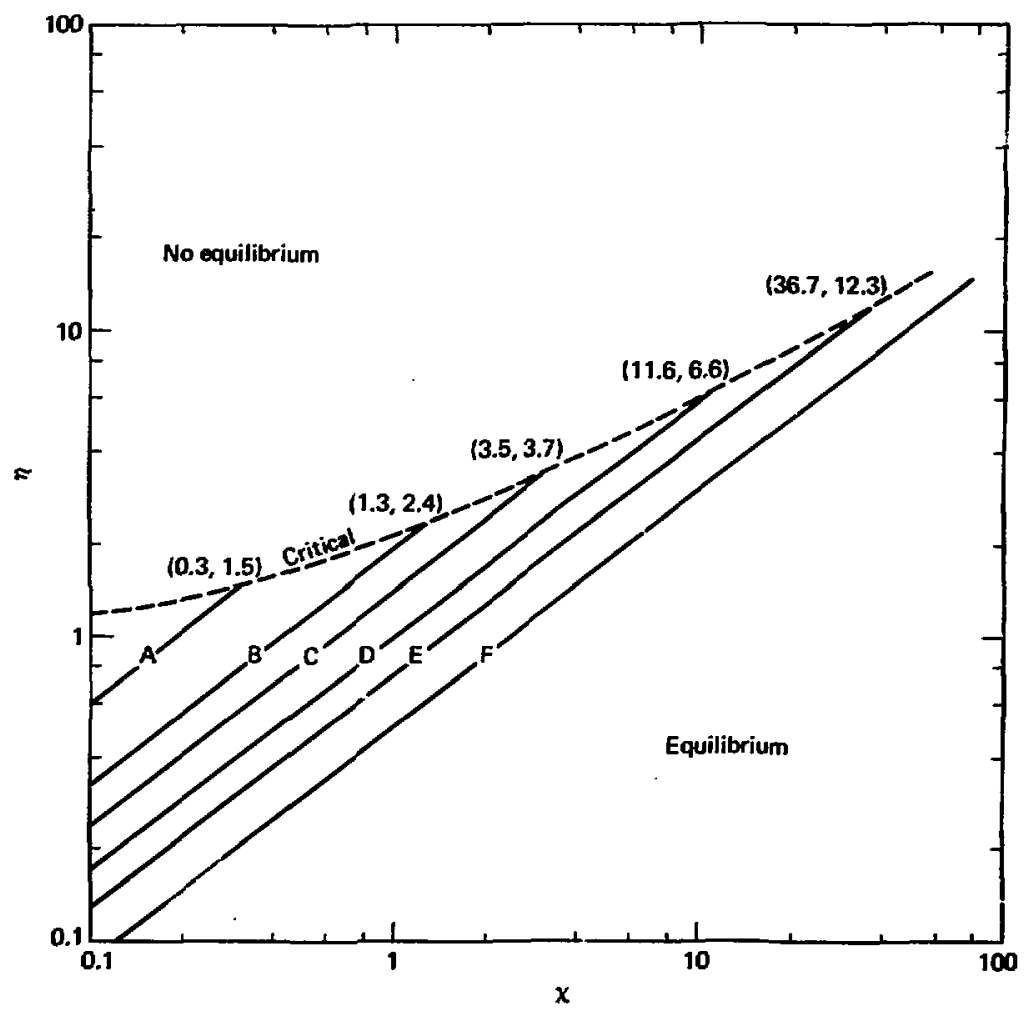

Figure 4.20--Crilical line for $s=1$ spheroids in $(x, \eta)$ space. Critical Bonner-Ebert aphere is the point $(x=0, \eta=1)$. Cooling sequence trajectories are constrained to be traight lines. Sequence $4 \mathrm{f}$ was not cooled to criticality. 


\section{CHAPTER 5}

ROTATING ISOTHERML COLLAPSE

\section{1) Introduction}

An interstellar cloud with insufficient internal and rotational energy to support itself against gravity will collapse nearly isothermally unti dust opacity in the dense central region inhibits the flow of cooling radiation (Winkler and Newtan 1980). In this chapter gravitational collapse of a rotating.. strictly isothermal cloud is considered. By ignoring nonisothermal effects for the moment, parameter space is kept manageably small.

Collapse is far more difficult to compute than equilibrium (chap. 4), because of the large spread of length and timo scales the system develops. The inclusion of angular momentum further complicates the task because systematic errors in angular momentum advection systematically change the evolution (Norman, Wilson, and Barton 1980). Indeed, computing a nonrotating collapse on a $2 D$ code rould be difficult, simply from a resolution standpoint.

As discussed in chapter 1, previous work on collapse has produced both $r i n g$ and disk solutions to identical problems. We believe discrepancies exist in the literature because of the numerical difficulties of computing such a highty convergent flow in more than one dimension. We hope to clarify the situation by reexamining collapse with the improved numerical methods outlined in chapter 3 . 
The questions we try to answer are:

(1) What are the generic features of collapse?

(2) Which states does collapse connect (i.e. disk or ring)?

A number of calculations have been run to answer these questions. The total angular momentum and its distribution were the primary quantities varied. In addition collapsc from overstable equilibrium was compared to collapse from a uniform spherical state. Constant volume and constant pressure boundary canditions were variously used. Before we describe specific evolutions, the following is a synthesis of the generic features of rotating isothermal collapse :

(1) runaway collapsc and flattening of the central rcgion of the cloud producing in approximately a freefall lime a large density contrast between the center and cdge of the cloud

(2) establishment of almost self-similar distributions of density and angular velocity in the equatorial plane of the cloud

(3) quasistationary isothermal shock fronts marking episodes of flow readjustment where collapse is supersonic

The distribution of angular momentum is found to be the key quantity in determining whether a ring forms, or whether the disk lopology is preserved. This is not surprising in view of the findings in chapter 4 . For those cases where a ring forms, features (1)-(3) are a dynamic backdrop for the process. Once a ring is established, however, feature (2) is no longer true. The distributions of density and angular velocity peak of $f-a x i s$. Those cases where a $r$ ing is not formed collapse to beyond the present method's ability to resolve the central structure. This also is not surprising since nonrotating isothermal collapse produces a singular, self-similar flow (Shu 1977). 
The total angular momentum appears to scale the evolutions rather than change them qualitatively. For instance, ring formation occurs either early or late in the collapse depending on the amount of angular momentum. Initial configuration (l.e., sphere or overstable equilibrium) seems to have little qualitative effect on the evolution as well. We find that a sphere assumes a near-equilibrium structure in the envelope in roughly one sound travel time across the cloud, and thereaster evolves qualitatively like collapse from equilibrium. The choice of boundary condition, that is constant pressure or constant volume, has little qualitative effect provided the center of the cloud evolves rapidly away from the edge. Eventually the boundary condition must be import + i, during the accretion phase, however this phase of the evolution is not covered by these calculations.

The above statements will now be supported by numerical examples.

\section{2) Collapse from Equilibrium}

A procedure for generating sequences of rotating, axisymmetric, isothermal equilibria was described in chapter 4. Collapse is calculated starting Irom the critical models of sequences $4 A, 4 C$, and $4 \mathrm{~B}^{\circ} \%$. A $42 \times 42$ grid was used for the first two, and a $22 \times 22 \mathrm{grid}$ for the last. These cases are a representative cross section of three cloud types: (1) nearly spherical, (2) flattened, and (3) toroidal. Cases 4A and 4C collapse to high central concentration, while case 4B" collapses to a hoop.

The onset of collapse is merked by a sudden flattening at the poles of spheroida $4 A$ and $4 C$, or above the ring center in $4 B^{\prime}$. Generally, soon after collapse begina zone resolution at the focus of the flow becomes inadequate. To set the stage for the higher resolution studies described in the next section, 
the last adequately zoned configuration for these cases are shown in $f$ igures 5.1 to 5.3. The clouds are not in equilibrium at this stage but continue to collapse. Comparing these figures to the critical models in $f$ igures $4.2,4.5$, and 4.6, we see high central concentration. Distributions of various quantities on the symetry axis and in the equatorial plane are shown for the thrce cases in Iigures 5.4 to 5.6 , respectively.

The power-law aspect of $\rho(R), \quad \sigma(R)$, and $D(R)$ for cases $4 A$ and $4 C$ is apparent in figures 5.4 and 5.5. Comparing $\rho(R)$ and $\cap(R)$ to their equilibrium counterparts in figures 4.9 and 4.10 , we see the plateau has diminished at the expense of the envelope, which preserves its slope. This feature appears entirely analogous to the collapse of the critical Bonner-Ebert sphere (Shu 1977). The bend in $\rho(z)$ in figure 5.4 a locates the foot of a developing shock front. The origin of this shock is discussed in the next section. It is a convergence effect in nonspherical, supersonic flow. Foree ratio distributions in the equatorial plane show the envelope has barely responded to the central coll apse.

The axisymetric collapse of an already formed $r i n g$, case $4 \mathrm{~B}^{\prime \prime}$, is of marginal interest because 3D effects rapidly become important (Norman and Wilson 1978). The peaked distributions of $\rho, \sigma$, and $\Omega$ on the equator are displayed in figures $5.6 a, b, c$. Force ratio distributions on the equator are included for further reference. 


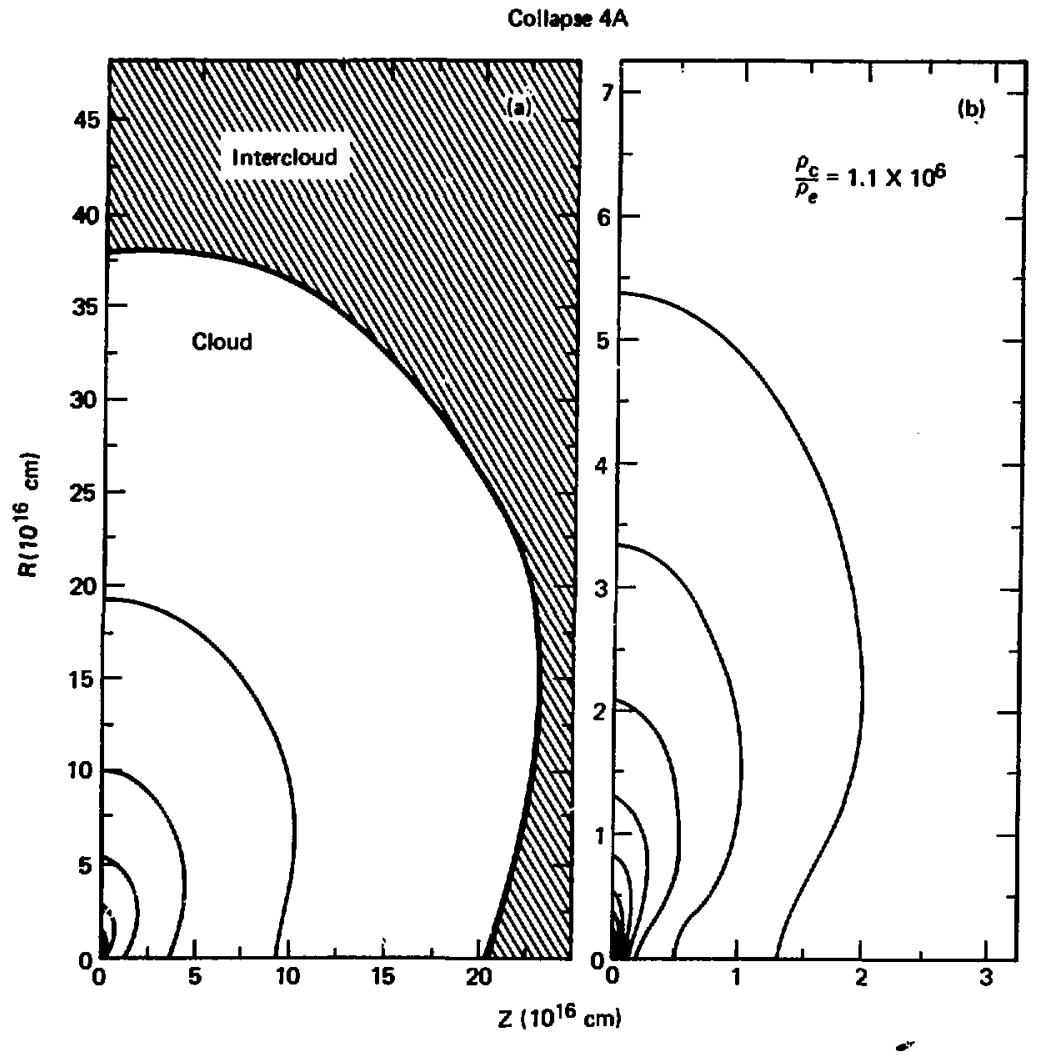

Figure 5.1--(a) collapse from equilibrium of a slowly rotating cloud, critical model $4 A$, shown in isodensity contours. (b) enlargement shows flattened central structure of last adequately zoned model. A $42 \times 42$ grid was used. 
Collepse 4C
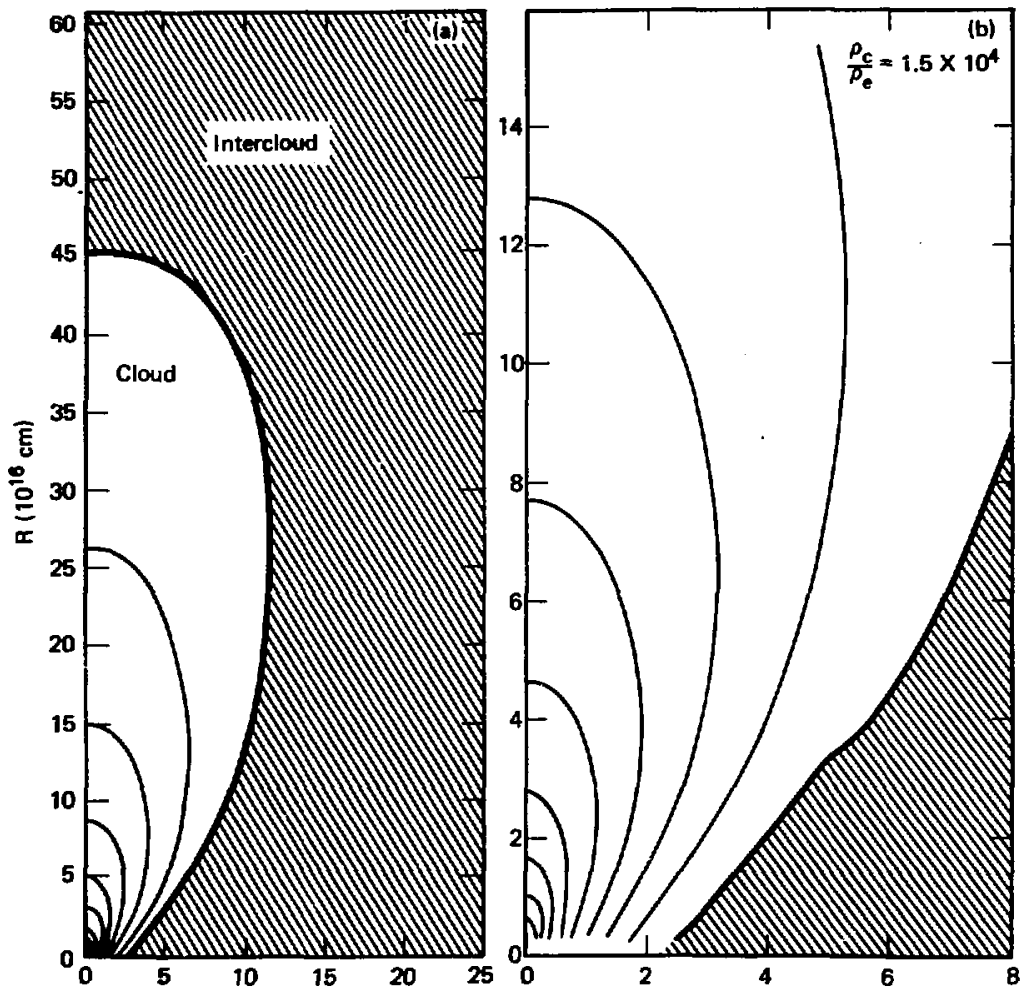

$Z\left(10^{16} \mathrm{~cm}\right)$

Figure $5.2-(a)$ Collapse from equilibrium of a rapidly rotating cloud, critical model $4 C$, shown in isodensity contours. (b) Enlargement shows central structure of last adequately zoned model. A grid of $42 \times 42$ zones was used. 
Collapse 48"

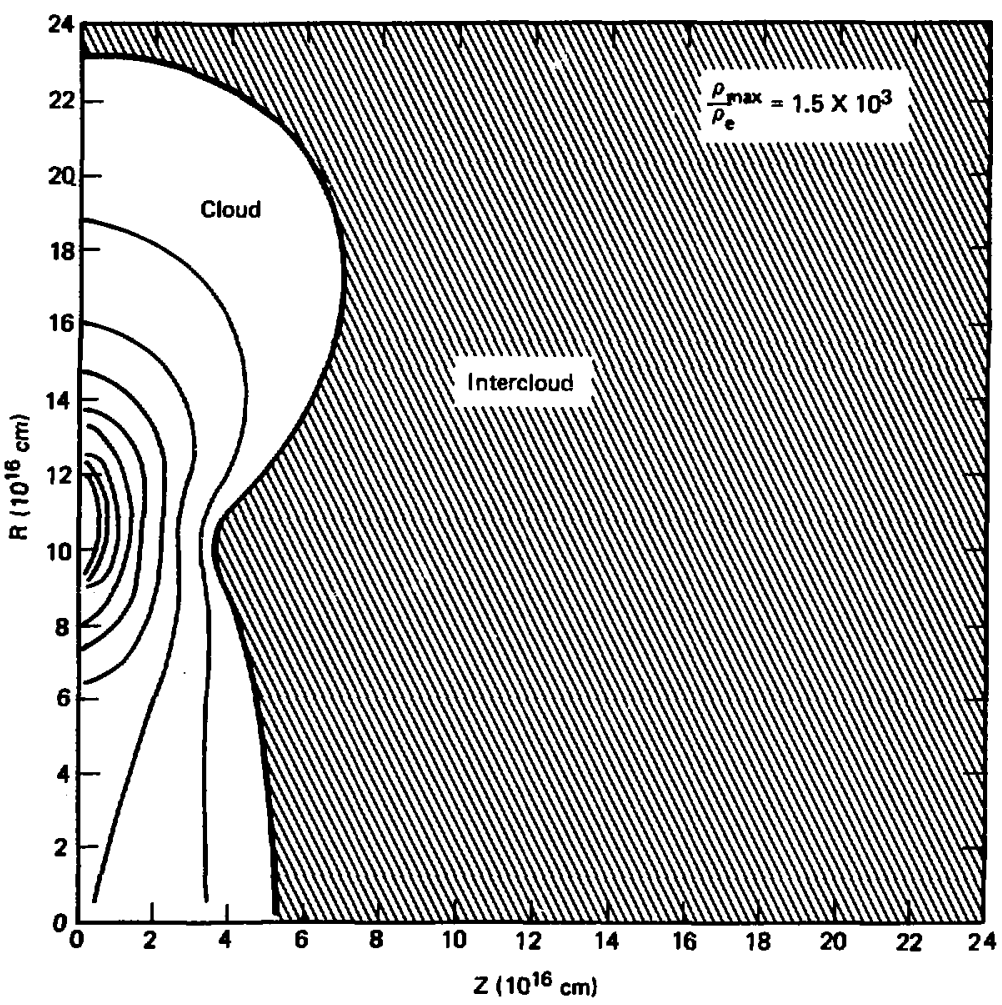

Figure 5.3-Collapse from equilibrium of a toroidal cloud, critical model $4 \mathrm{~B}^{\prime \prime}$. Shown in isodensity contours. The $22 \times 22$ grid became inadequate at thit $t$ ine. 

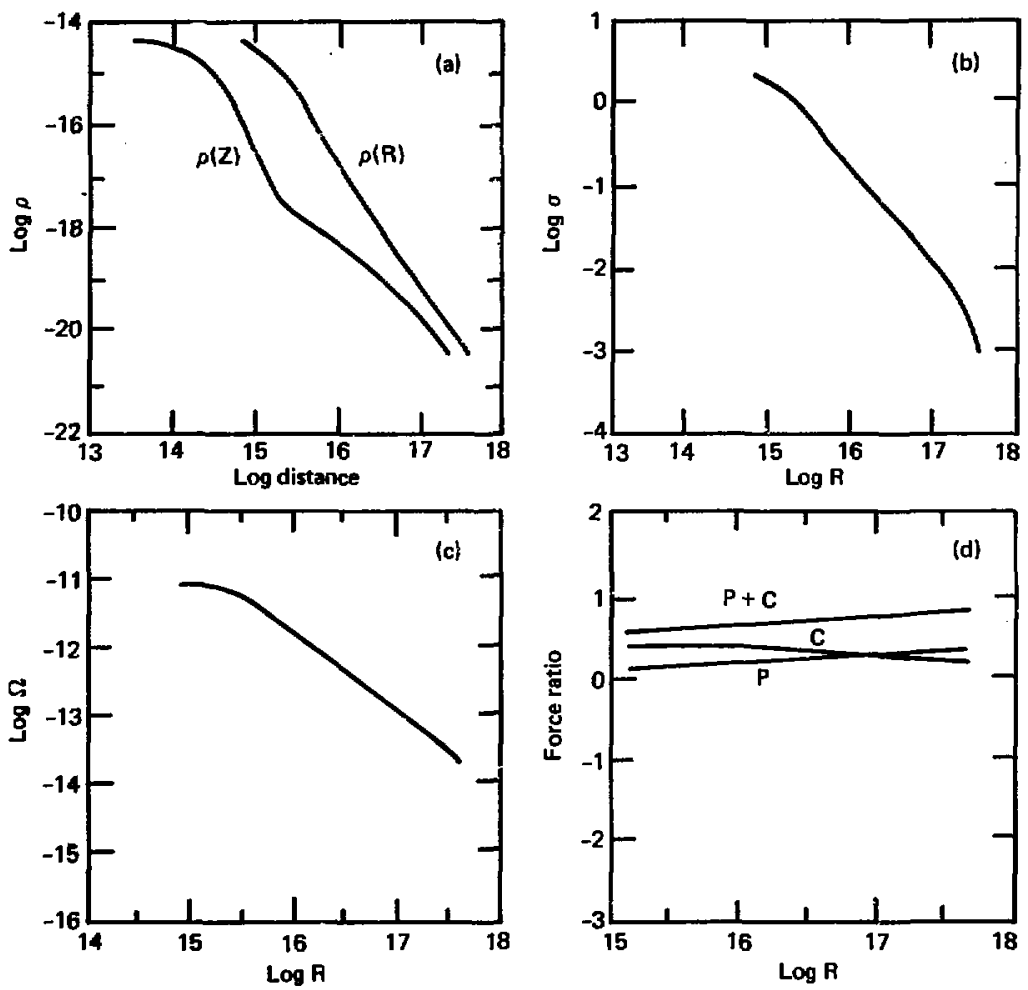

Figure 5.4--Distribution of (a) mass density, (b) surface denaity. (c) ancular velocity, and (d) ratio of pressure to gravilational force (P). ratio of centrifugal to gravitational force (C), and their sum in the equatorial plane. 

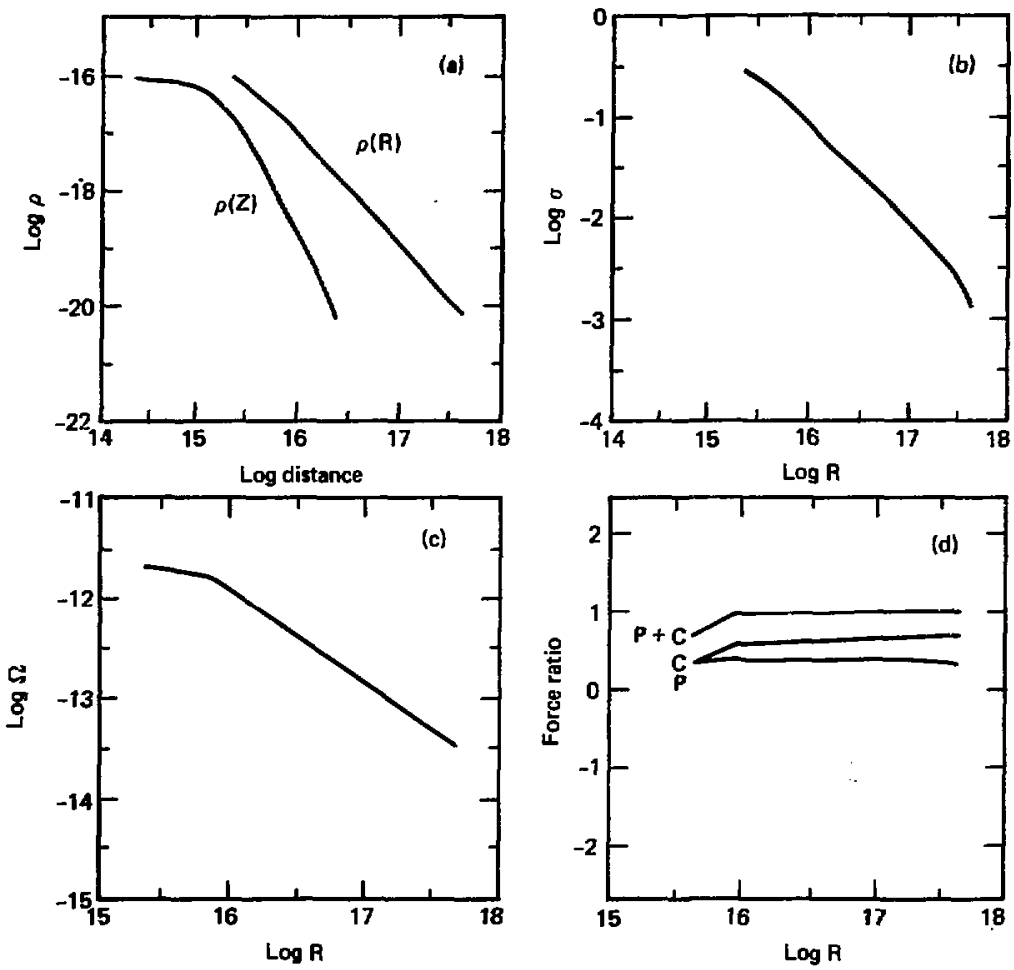

Figure 5.5--Digtribution of various quentities for the last well-zoned model of collapse 4C. 

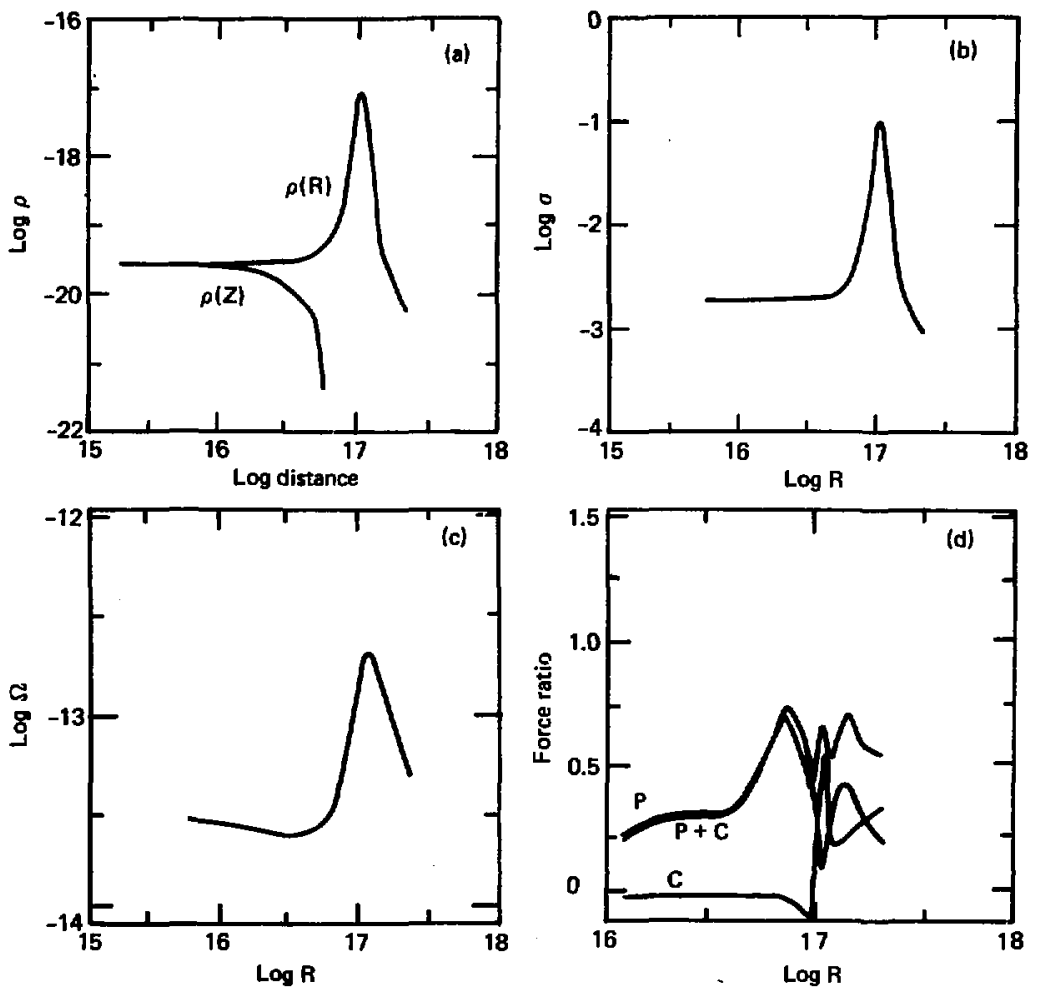

Figure 5.6--Distribution of various quantilies for the last well-zoned model of collapse $4 B^{\prime}$. 


\section{3) Collapse of Rigidly Rotating Spheres (s = 1)}

Historically, the first initial conditions chosen were uniform, spherical clouds in rigid rotation (angular momentum distribution parameter $\mathbf{s}=1$ as defined in sec, 4.3), confined by a constant spherical volume (Larson 1972). Table 5.1 lists the parameters for a series of such initial models. Several of them match initial models calculated by Black and Bodenheimer (1976). Meridional velocities are initially zero. Collapse proceeds inside a spherical volume of radius $R_{0}$. Since the evolutions are kept isothermal the collapse problem has no scale. The scaling laws have been given in chapter 4. For convenience the scale given in table 5.1 will be retained.

Evolutions 5A, 5B, and $5 E$ are later compared to Black and Bodenheimer's (1976) models $1 \mathrm{~A}, 1 \mathrm{~B}$, and $1 \mathrm{D}$, respectively. Although their code capability included the thermodynamics and the radiation hydrodynamics necessary for a proper treatment of core tormation, that physics was not excercised in their evolutions because they found ring tormation in the isothermal phase. Therefore our respective calculations can be directly compared. 
TABLE 5.1

RIGIDLY ROTATING SPHERE PARAMETERS $\therefore$.

$$
\begin{gathered}
\text { Mass: } \mu=\mu_{0} \\
\text { Radius: } R_{0}=7 \cdot 10^{10} \mathrm{~cm} \\
\text { Sound Speed: } a=2 \cdot 10^{4} \mathrm{~cm} \mathrm{~s}^{-1} \\
\text { Density: } \rho_{0}=1.38 \cdot 10^{-18} \mathrm{~B} \mathrm{~cm}^{-3}
\end{gathered}
$$

Boundary Condition: Constant Volume

\begin{tabular}{cccc} 
Designation & $J\left(10^{54} \mathrm{~g} \mathrm{~cm} \mathrm{~s}^{-2}\right)$ & $\mathrm{s}$ & 7ones \\
\hline $5 \mathrm{~A}$ & 0.60 & - & $48^{2}$ \\
$5 \mathrm{~B}$ & 1.20 & 1 & $48^{2}$ \\
$5 \mathrm{C}$ & 1.68 & 1 & $48^{2}$ \\
$5 \mathrm{D}$ & 2.08 & 1 & $48^{2}$ \\
$5 \mathrm{E}$ & 2.40 & 1 & $22^{2}$
\end{tabular}


Evolutions 5A-5C were computed with two gridmotion schemes. The tirst kept the outer boundary of the grid motionless (prescripticta, sec. 3.6). Soon at ter collapse began it became obvious that extremely fine zone resolution was needed at the center to resolve the flow. The second scheme allowed the grid boundary to move inwards through the virtually static envelope (prescription b. sec. 3.6), so that the entire grideffectively zoomed in on the center of the cloud. In this case material left the grid supersonically so no signals propagated upstream to the center of the cloud. The only physical effect of the second scheme is gravitational, and that appears to be a small effect in these cases.

Meridional sections of the cloud at various stages of collapse for all calculations are archiyed at the end of this chapter. Rather than a point by point description of each frame, we concentrate on the generic features. A detailed description of case $5 B$ has been given by Norman, Hilson, and Barton (1980).

\section{Generic Features}

Cases 5A, 5B, 5C, and 5D evolve to very high central densities, while case SE oscillates about an equilibrium. Apparently only the first four are unstable in this sense, although case 5D showed a strong rebound before evolving to high central density. Of these four the predominant feature is a runaway collapse and flattening of the central regions of the cloud. As collapse proceeds, the density contrast between the center and edge of the cloud becomes many orders of magnitude. As a result, the evolutionary timescale of the center becomes much shorter than a sound-traversal time of the envelope. Subsequent attention is directed to dynamics at the center of the cloud. 
By roughly one freefall time after collapse begins the cloud has flattened into disk (e.g., frame 5A.2). The vertical structure of the disk becomea nearly hydrostatic as the evolutionary timescales in the two perpendicular directions diverge. Flow down the axis is supersonic and is decelerated by a planar shock front lying nearly parallel to the equator above the disk (e.g.. frame 5A.4). The shock front forms because supersonic material collides with the temporarily pressure supported disk. The height of the shock front above the equator is always nearly one equilibrium scale height for an isothermal disk.

$$
h_{\mathrm{eq}}=\frac{a^{2}}{4 \pi \mathrm{G} \sigma} .
$$

where $\sigma$ is the surface density of the disk, and $a$ is the sound speed.

Now $\sigma$ changes as material flows to the center radially. Although the radial flow is subsonic, o changes quite rapidly near the rotation axis due to convergence. Eventualy the new surface density distribution forces a dynamic readjustment of the disk height, and a new flattening phase ensues in the center-most part of the cloud (e.g., frames 58.3-5B.8). The process accurs in episades, so that a sequence of shock fronts are produced in increasingly smaller portions of the disk center at the end of each flattening phase (e.g., frames $5 B .8,5 B .12)$.

Clouds of low angular momentum evolve to higher densities before shocking than clouds of high angular momentum (c.f.. frames 5A.5, 5D.1). While the location and strength of each shock front may depend on initial canditions, they mist occur whenever supersonic material encounters a region in approximate force balance. Flattening, due here to rotation, leads to temporarily supported 
regions even though the gas is isothermal. The shock fronts are an effect of nonspherical collapse.

An interesting feature of collapse is that in the center of the cloud $\frac{d \ln n}{d \ln \rho} \sim \frac{1}{2}$.

Figure 5.7 shows the run of $Q$ versus $\rho$ for case $5 B$, and is representative of the four cases. The bumps in the data where the slope is not $1 / 2$ indicate readjustment of disk structure accompanying the flattening phases previously described.. In appendix $B$ we demonstrate that equation (2) is a consequence of mass and angular momentum conservation in flattened isotherinal systems.

A turther generid. feature of collapse is the establishment of approximately power-law distributions of density, surface density, and angular velocity on the equator. Figures 5.8a,b,c show these quantities for case 50 at the end of the computed evolution, and are representative of the four. The general impression of collapse is that under the isothermal assumption arbitrarily high central densities could be reached provided grid resolution was fine enough to follow it. We know this is the case without rotation. Can this occur with rotation?

Ultimately the extreme flattening and concentration of the cloud center prohibits further integration. Typical density ratios between center and edge are eight orders of magnitude. If the evolutions were scaled to collapse from typical interstellar density (say a diffuse $H I$ cloud with $\mathrm{n} \sim 20 \mathrm{~cm}^{3}$ ). then the material would only now become nonisothermal at the center.

As mentioned, successively smaller portions of the cloud undergo an episodic flattening. The majority of the disk approaches forse balance, however, as hown in $t$ igure $5.8 \mathrm{~d}$. Notice the total ratio exceeds unity for $R \&$ 


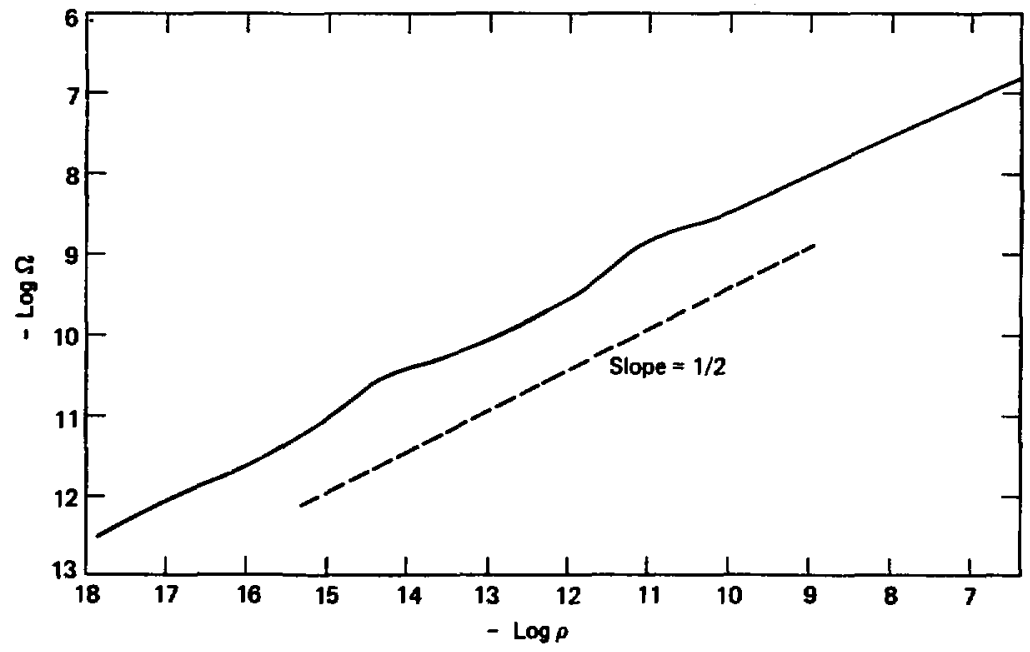

Figure 5.7--The central angular velocity as a function of central density in evolution 5B. Bumps accompany shock front formation and disk flattening phases. 

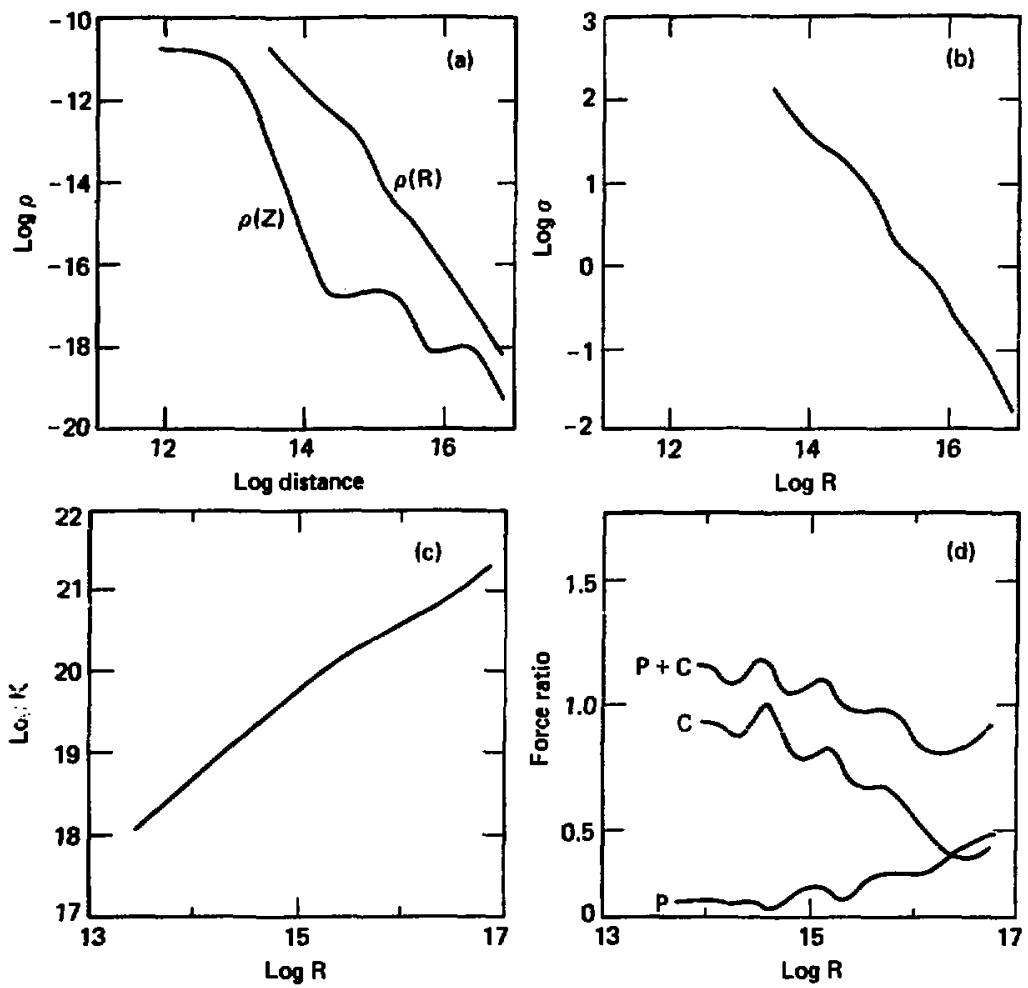

Ficure 5.8-Distribution of various quantities (see fig. 5.4) for last adequately-zoned model of collapse 5D. (a) $\rho(Z)$ shows multiple shock fronta. (b,c) Quite teatureless distributions of surface density and specific angular momentum. (d) Pressure plus centrifugal force exceed cravity in the equatorial plane, indicating imminent centrifugal rebound. 
$10^{18} \mathrm{~cm}$, indicaling the disk has overshot equilibrium. Black and Bodenheimer (1976) have argued that centrifugal rebound perturbs the disk sufficiently to initiate ring tormation. There is no doubt that centrifugal rebound will perturb 1:s disk. but whether the perturbation grows to the point of gravif.. $11 y$ binding itself is another matter; one that must be calculated numer $y$ since it is a problem in the nonlinear growth of vave. We cannot integrate far enough in time to ussess this at present.

An alternate approach that is taken up in chapter 7 is to construct disk solutions to an idealized collapse, and test them for local stability to axisymetric modes by a linear analysis. Presumably the cloud would not reach the singular state if it was unstable to the growth of ring modes but would concentrate into a ring or rings. We show that a singular, power-law thin disk in equilibrium is a consistent final state for ideal collapse from our initial conditions. We interpret the runaway collapse, complete with episodic llattening and shock formation, as simply reverberations the cloud center undergoes as it evolves to a singular state. Under some restricting assumptions, the analysis predicts that evolutions 5A-5D will eventually become unstable to ring formation.

Evolutions $5 \mathrm{~A}$ and $5 \mathrm{~B}$ disagree with evolutions $1 \mathrm{~A}$ and $1 \mathrm{~B}$ of $\mathrm{Black}$ and Bodenheimer (1976). They $f$ ind $r i n g$ formation by the time pectral $\sim 10^{-12} \mathrm{~g} / \mathrm{cm}^{3}$ for case $1 \mathrm{~A}$, and $\rho_{\text {central }} \sim 10^{-14} \mathrm{~g} / \mathrm{cm}^{3}$ for case $1 B$; whereas evolutions $5 \mathrm{~A}$ and $5 B$ have evolved to $\rho_{c e n t r a l} \geq 10^{-10} \mathrm{~g} / \mathrm{cm}^{3}$ without forming rings. We feel the numerical diftusion of angular momentum implicit in their angular momentum advection technique has hurried the onset of ring formation as discussed by Norman, Milson, and Barton (1980). 
Case 5E does not undergo runaway collapse but oscillates about an equilibrium, albeit one confined in a fixed volume rather than by a pressure. As such, the calculation has no bearing on ring formation in rotating collapse. Nevertheless, Bodenheimer and Tscharnuter (1979) ran comparison calculations of this case to address the "ring controversy". Figure 5.9 shows oscillations of the central density as a function of time, and agrees very well in detail to figures $1 \mathrm{a}$ and $\mathrm{lb}$ of the comparison paper. The dynamic features of our calculation matched their description quite well, however their interpretation of the evolution vis. a vis. rings is quite different than our own. We see mild of $-a x i s$ density enhancements (amplitude 25 percent) that are clearly sound waves reflecting off of the outer boundary. After the oscillations damp the boundary maintains the of f-axis density peak by its proximity.

To show that the of $f$-axis density peak is a boundary effect, the evolution was repeated with a constant pressure boundary condition: $P_{x}=5.74 \cdot 10^{-10}$ dyne $\mathrm{cm}^{-2}$ (equal to the initial pressure of the cloud). The cloud is stable even with the addition of boundary pressure, as the sequence of meridional sections show. Frame $5 E^{\prime} .2$ shows the cloud immediately flattens and extends beyond $\mathrm{R}=$ $7 \cdot 10^{16} \mathrm{~cm}-$-the radius of the fixed volume in run $5 \mathrm{E}$. Ring transients are clearly seen in frames $5 E^{\prime} .4$ and $5 E^{\prime} .8$.

A stable ring is untikely on two counts: (1) the cloud scales very nearly to an equilibrium we have already calculated-the critical model in sequence $C$, which has a monotonic density distribution, and (2) the classical ring instability was shown to be excited above $\beta \sim 0.40$ (section 4.5 .1 ), however here $\beta \sim 0.228$. Therefore we consider calculations $5 \mathrm{E}$ and $5 \mathrm{E}^{\prime}$ irrelevant to the "ring controversy". 


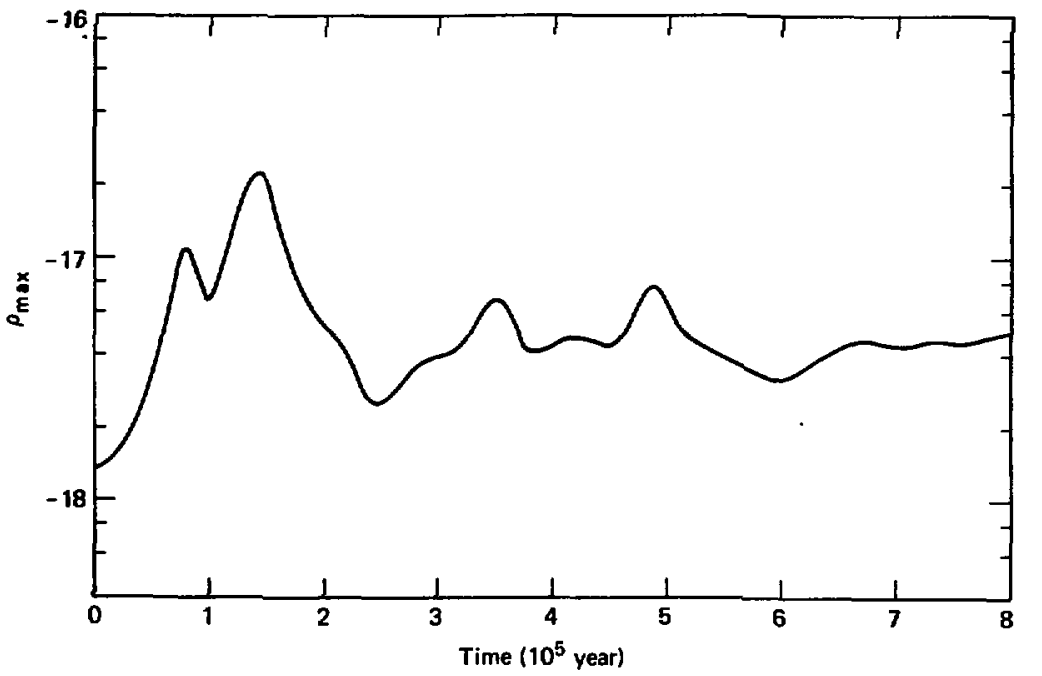

Figure 5.9-Central density as a function of time for evolution 5E. 
5.4) Collapse of Differentially Rotating Spheres $(s * 1)$

The distribution of angular momentum was found to have the largest effect on the evolution of a rotating collapsing cloud. Specifically, ring formation was found for values of the distribution parameter $s<1$, but no $r$ ing formation for $s>1$. We point out that ring formation may eventually occur in the collapse of $s>1$ clouds, but the singular nature of the collapse in those cases prevents us from integrating far enough to tell. Table 5.2 ists the parameters for two representative clouds differing only in the distribution of angular momentum. A constant pressure boundary condition was used. 
TABLE 5.2

DIFFERENTIALLY ROTATING SPHERE PARAMETERS

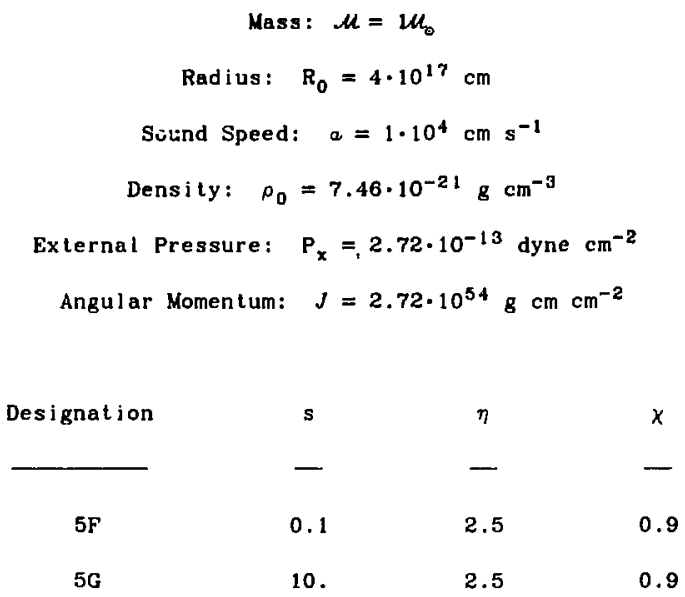

Case 5F forms a ring very early in the evolution. Frame 5F.1 shows the center of the cloud after roughly one freefall time. A shock front forms in the vicinity of $\mathrm{Z}=2 \cdot 10^{18} \mathrm{~cm}$. Centrifugal force begins to halt the radial flow of material to the center. Frames $5 F .2$ to $5 F .5$ show a centrifugal rebound of the disk which precedes $r$ ing formation, seen in trame 5F.6. The $r$ ing then collapses on itself as criticality is reached; frames $5 F .7$ and $5 F .8$.

Figure 5.10 shows the distributicns of $\rho, \sigma, n$, and the force ratios just prior to ring formation. The large centrifugal force ratio is offset by the low pressure ratio, summing to approximately unity. Thus ring formation occurs in 

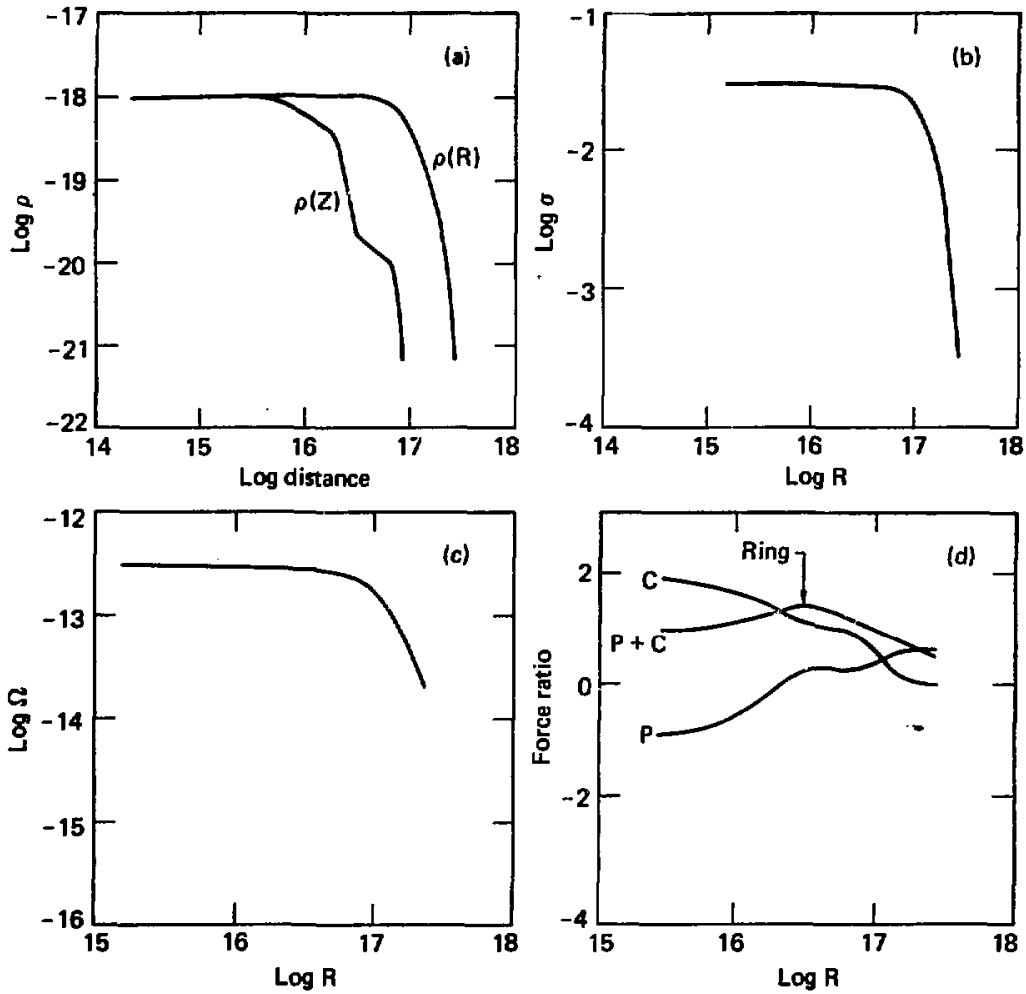

Figure 5.10-Distribulion of various quantities just prior to ring formation in evolution 5.F (matches Irame $5 F .2$ ). 
approximate force balance. The $r$ ing forms at the position of the maximum in the lotal force ratjo curve.

Evolution 5F contirms the semianalytic model of ring formation described by Tokline (1980). However his statement that ring formation is insensitive to the distribution of angular momentum is incorrect, as evolution $5 \mathrm{G}$ now demonstrates.

Case 5G collapses to a central density of greater than $10^{-12} \mathrm{~g} \mathrm{~cm}^{-3}$ in slightly greater than a freefall time. Collapse in the center proceeds without shock formation as it is nearly spherical. Frames 5G.1 to 5G.3 show the final computed state of the cloud in three different magnifications, and reveal a very centrally condensed configuration. Notice how the ouler contours in trame 5G.1 resemble the $s=10$ equilibrium spheroid, case $4 B^{\prime}$.

Figure 5.11 shows the distributions of $\rho, \sigma, \Omega$, and the force ratios at this time. The virtual structureless nature of the density and angular velocity, and the small force ratios indicate the entire cloud is far from equilibrium. Core formation would halt collapse in a realistic situation when the density was high enough to retard the flow of cooling radiation. This is described in the next chapter. 

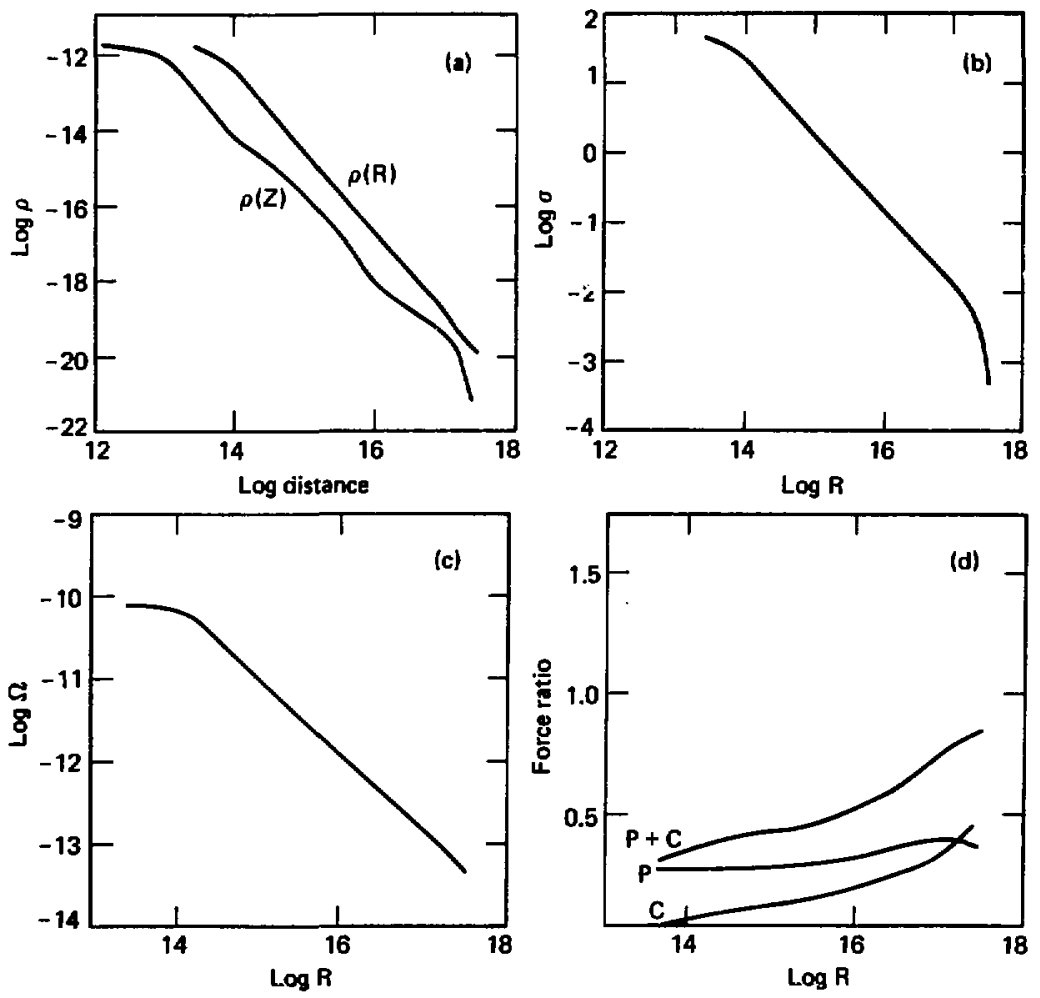

Figure 5.11--Distribtution of various quantities for the last adequate $1 y$-zoned model of evolution $5 G$ (matches frames 5G.1-5G.3). ( $a, b, c$ ) Nearly self-similar distributions od $\rho, \sigma, \Omega$ in the equatorial plane. (d) Force ratio shows the cloud is far from equilibrium. 
ACHIVE OF GRAPHICAL OUTPUT FOR COLLAPSE EVOLUTIONS

The following frames show a history of one quadrant of the cloud for evolutions 5A, 5B, 5C, 5D, 5E, 5E', 5F, and 5G. The rotation axis is horizontal. The solid(dotted) lines are isocontours of mass density(specific angular momentum). The levels step down by a factor of 2 from the maximum value RHOMAX(KMAX). The arrows e t $\therefore$ velocity field and define the the grid resolution. VMAX is the largest velocity. All numbers are in egs unit. Note the changing scale. 
Evolution 5A 


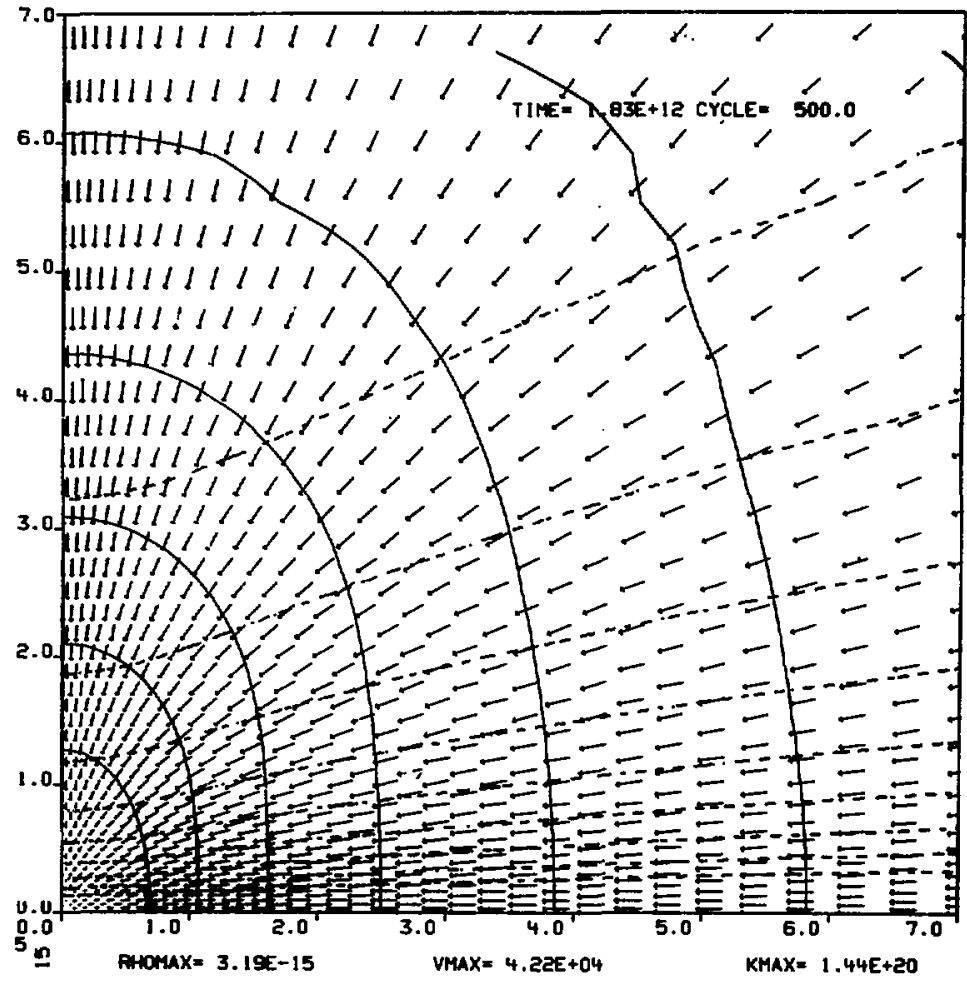

w

Frame 5A. 1 


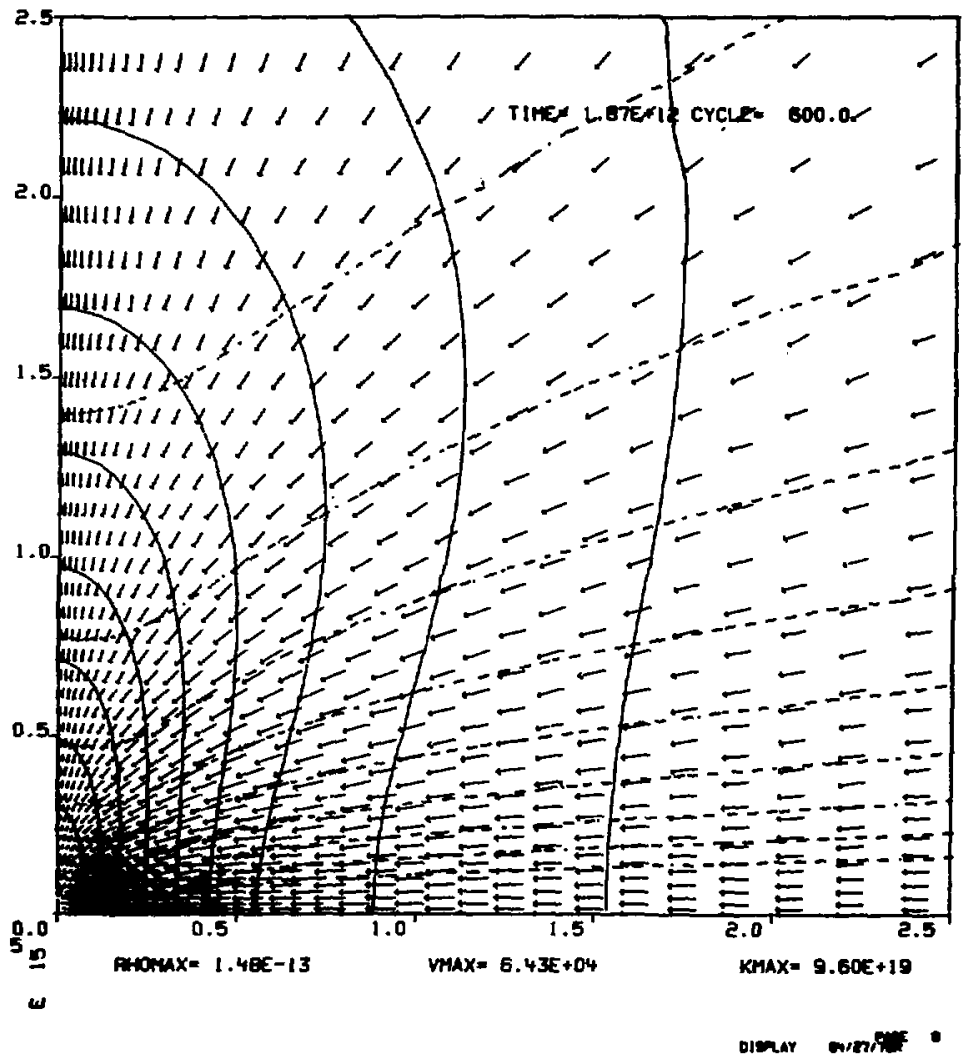

Frame 5A.2 


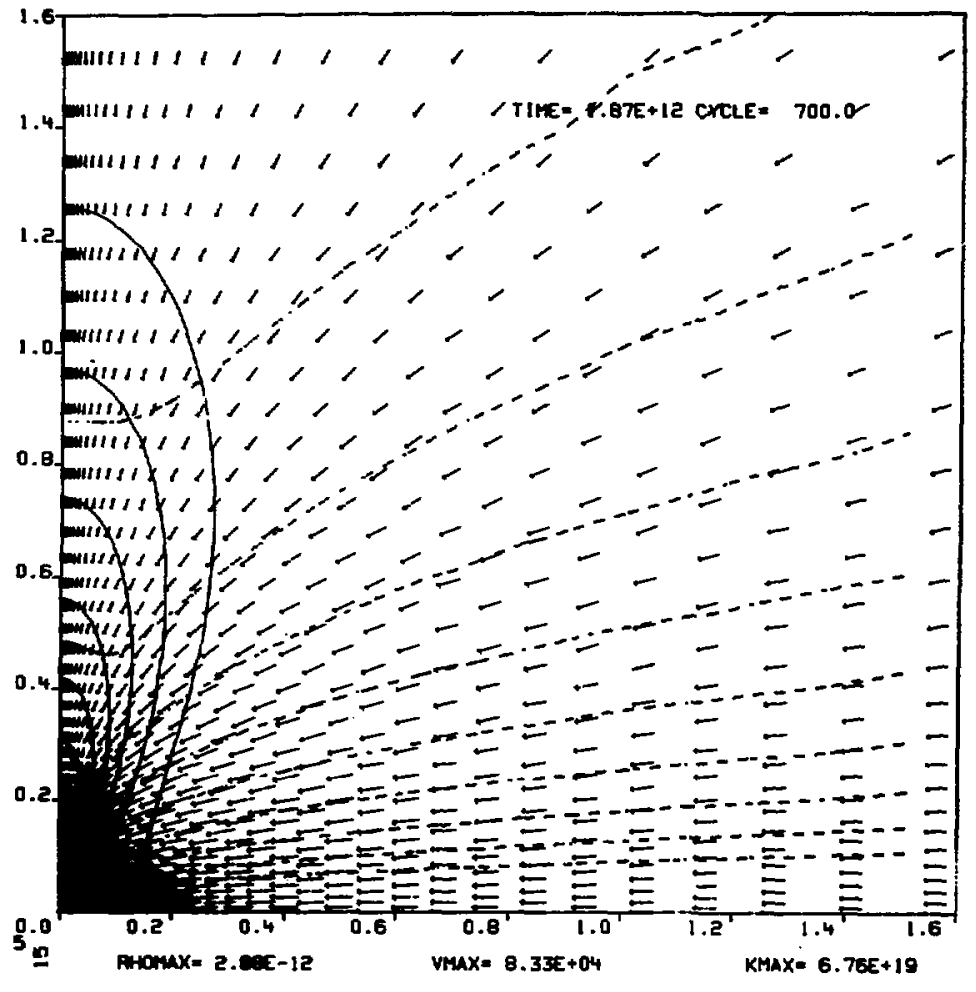

$\omega$

dienar minficf

Frame 5A.3 


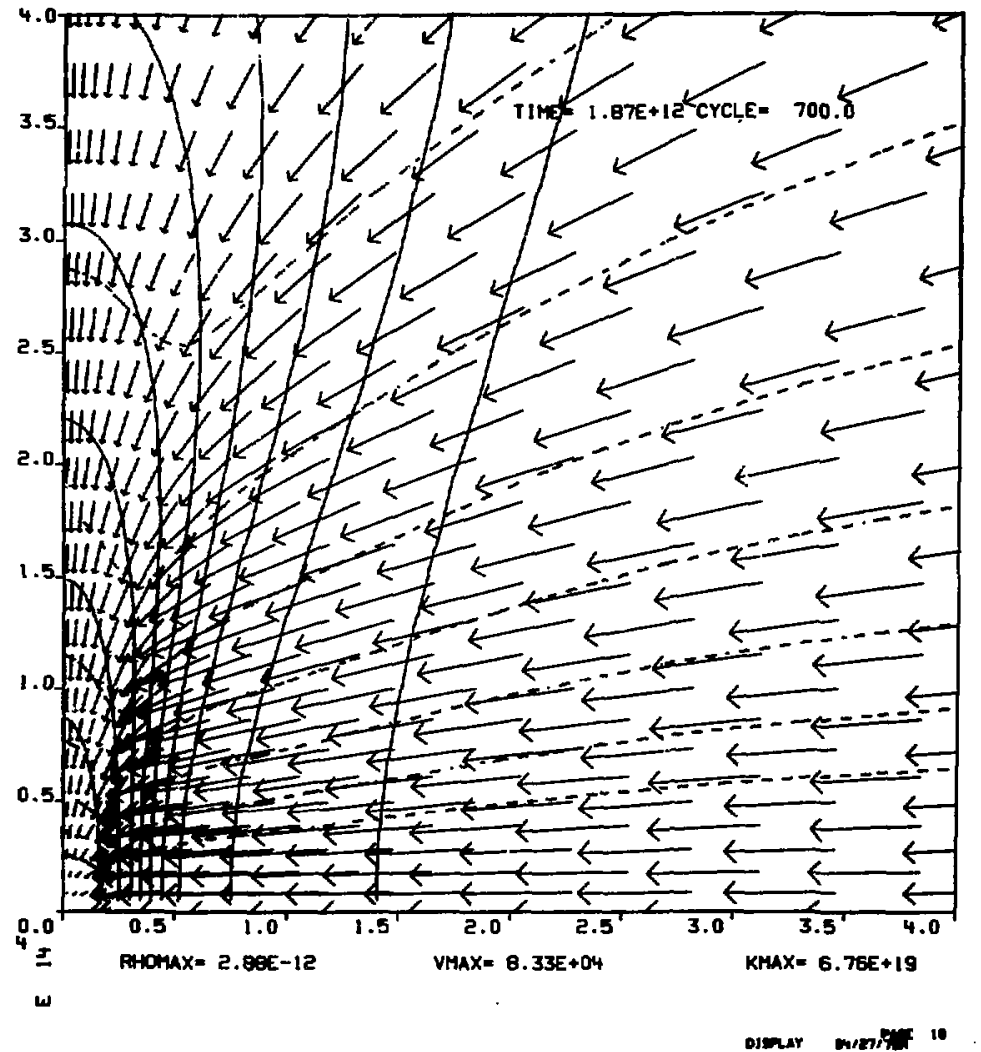

Frame $5 A .4$ 


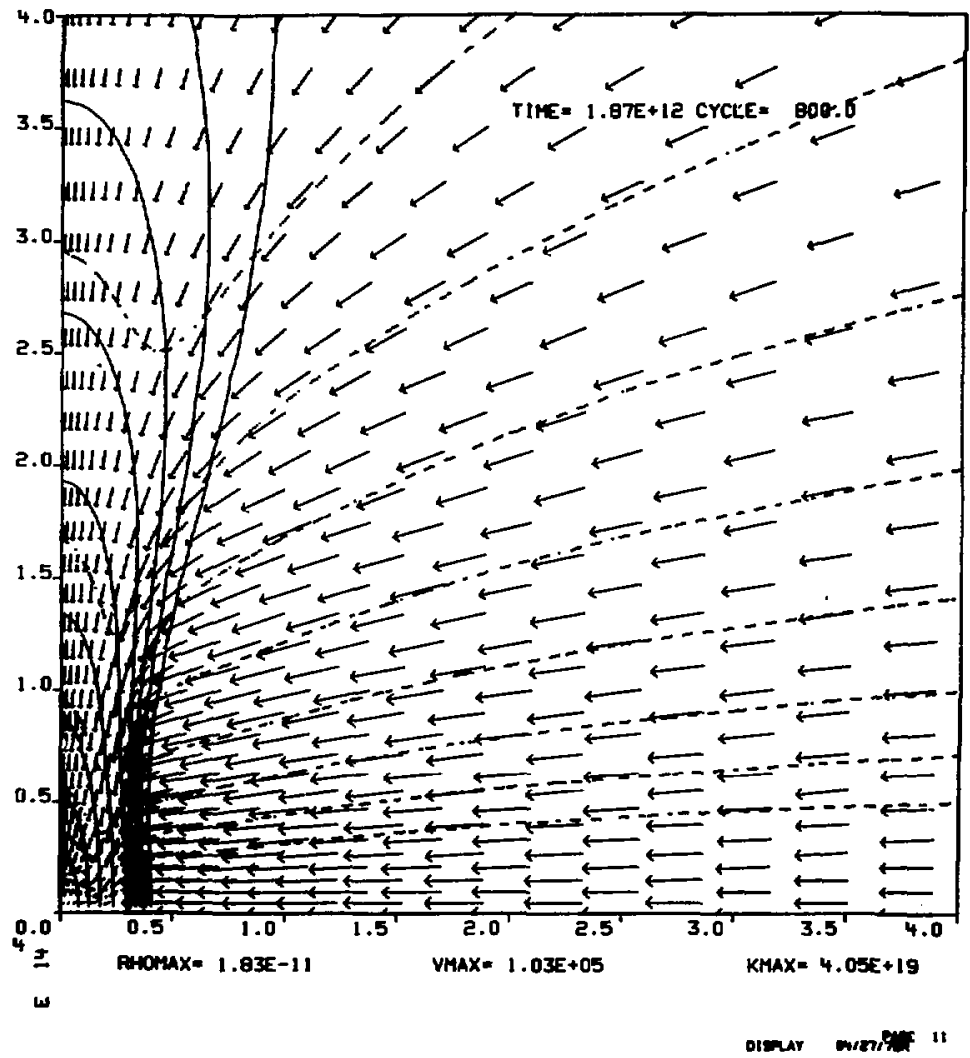

Frame $5 A .5$ 


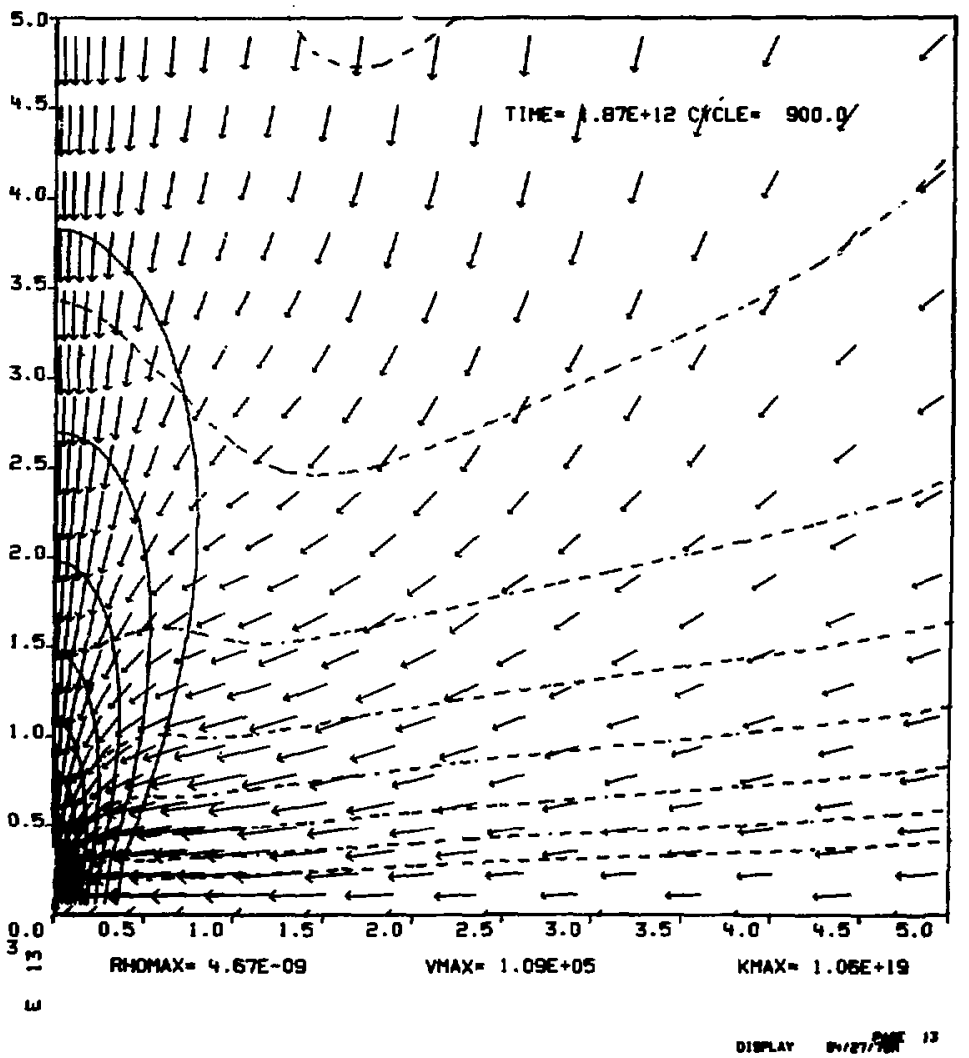

Frame 5A.6 
Evolution 5B 


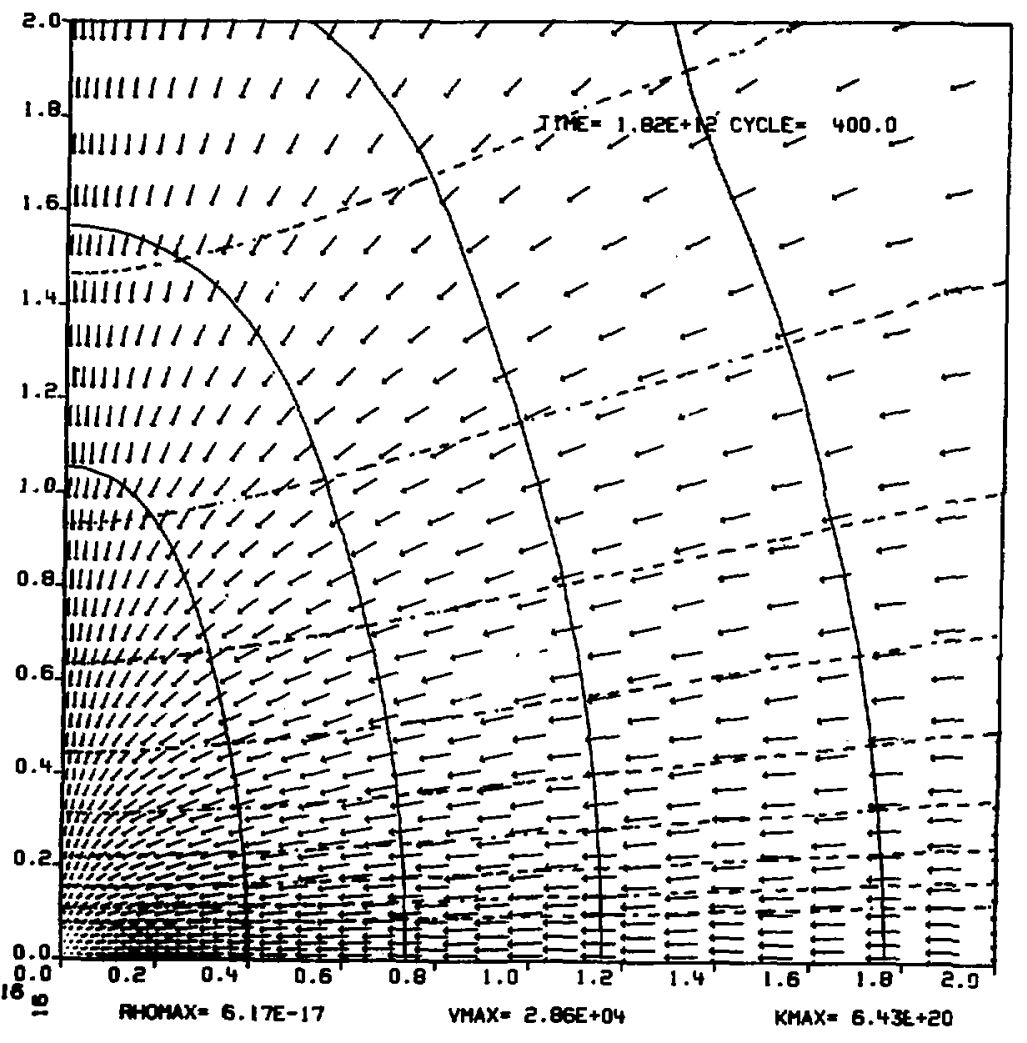

$\boldsymbol{\omega}$

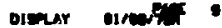

Frame 5B.1 


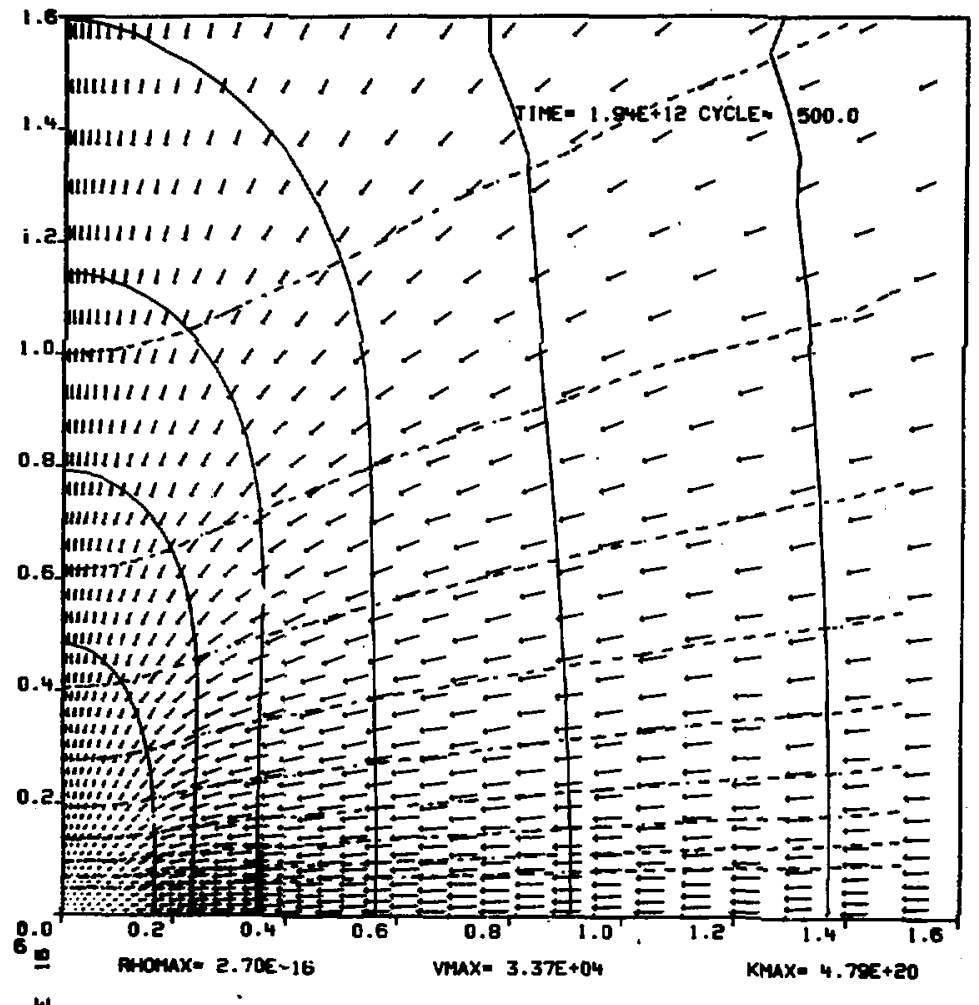

olemar mictifit

Frame 5B.2 


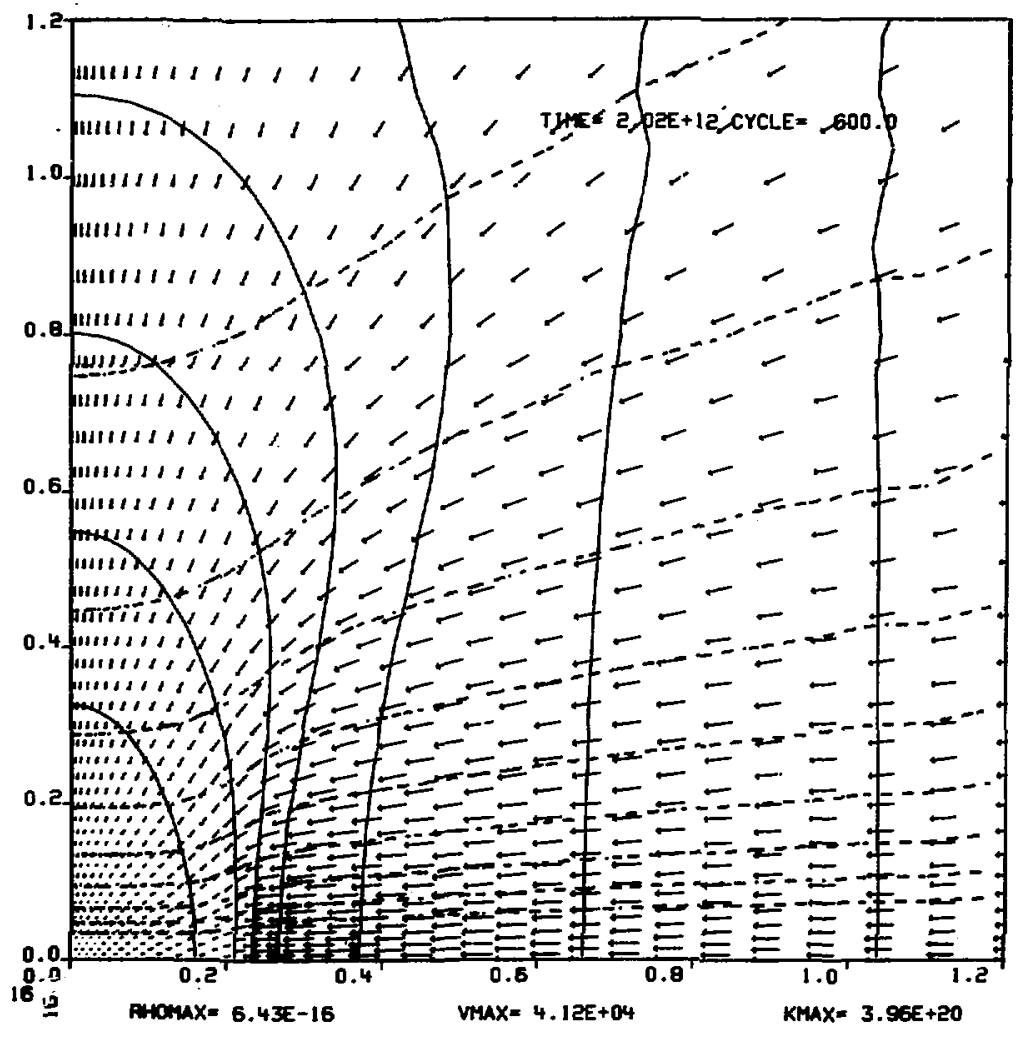

w

olemar oldenter 13

Frane 5B.3 


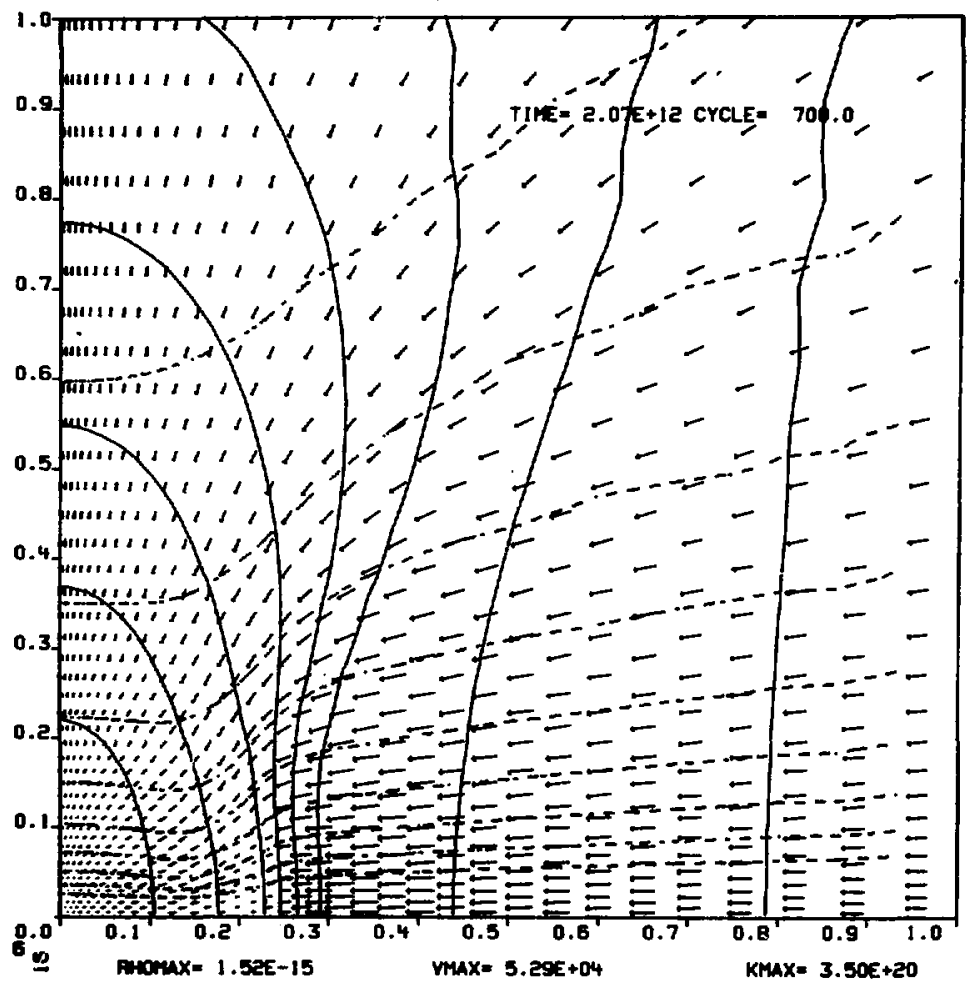

w

oterar enverifict.

Frane 5B.4 


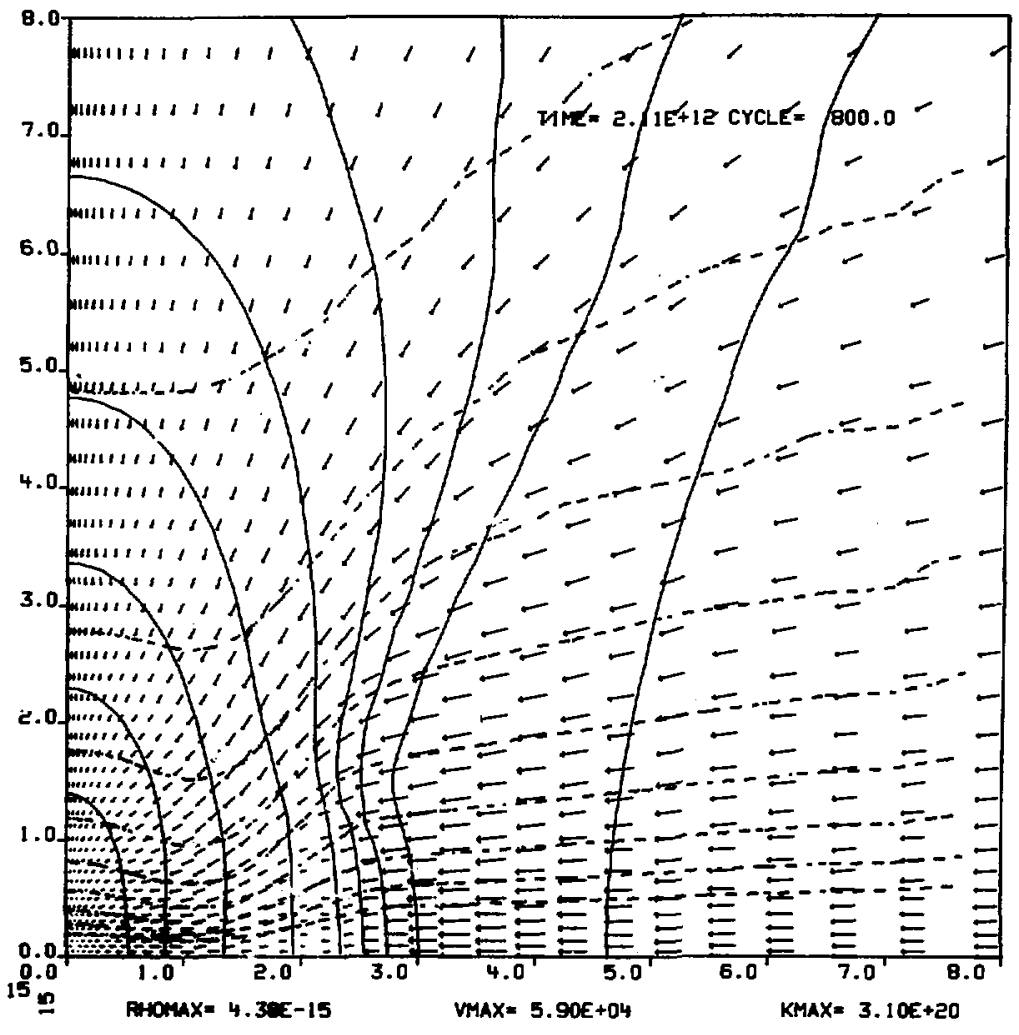

w

olshar eirevthe 17

Frame 5B.5 


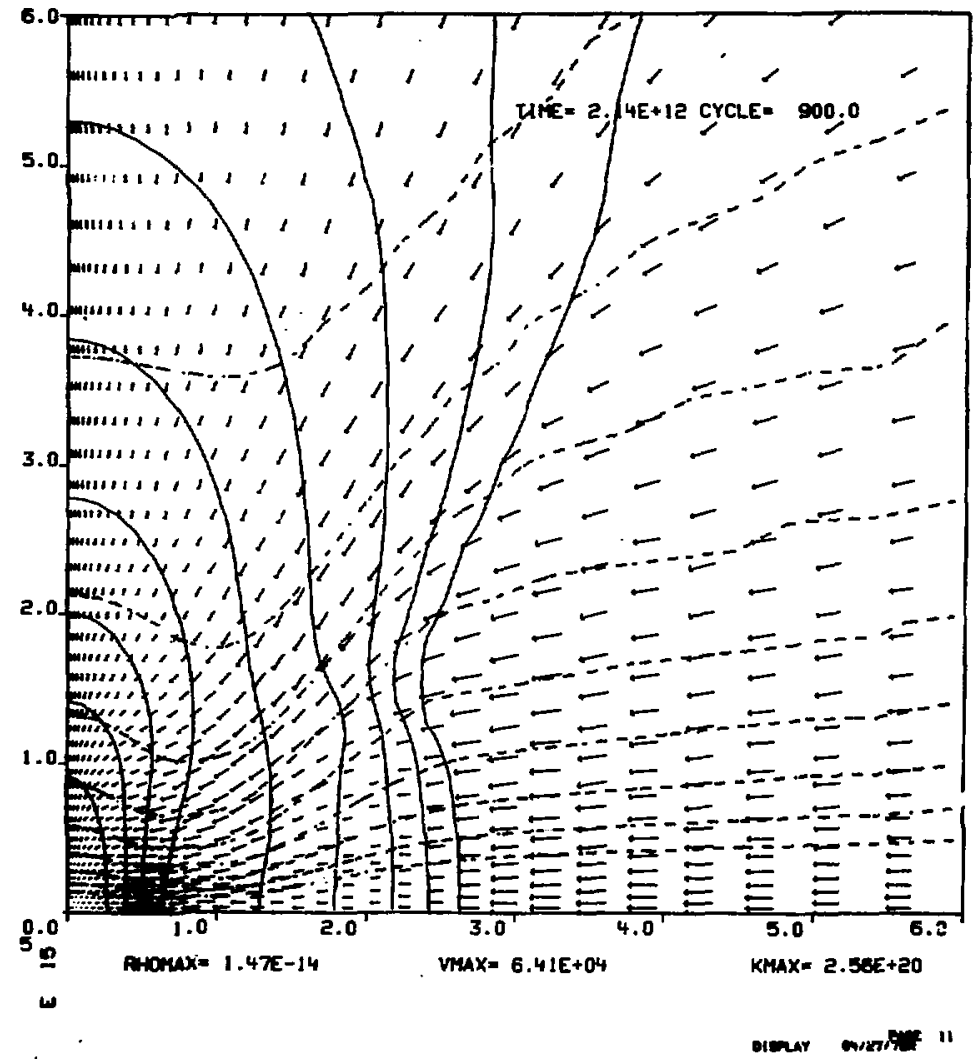

Frame 5B.6 


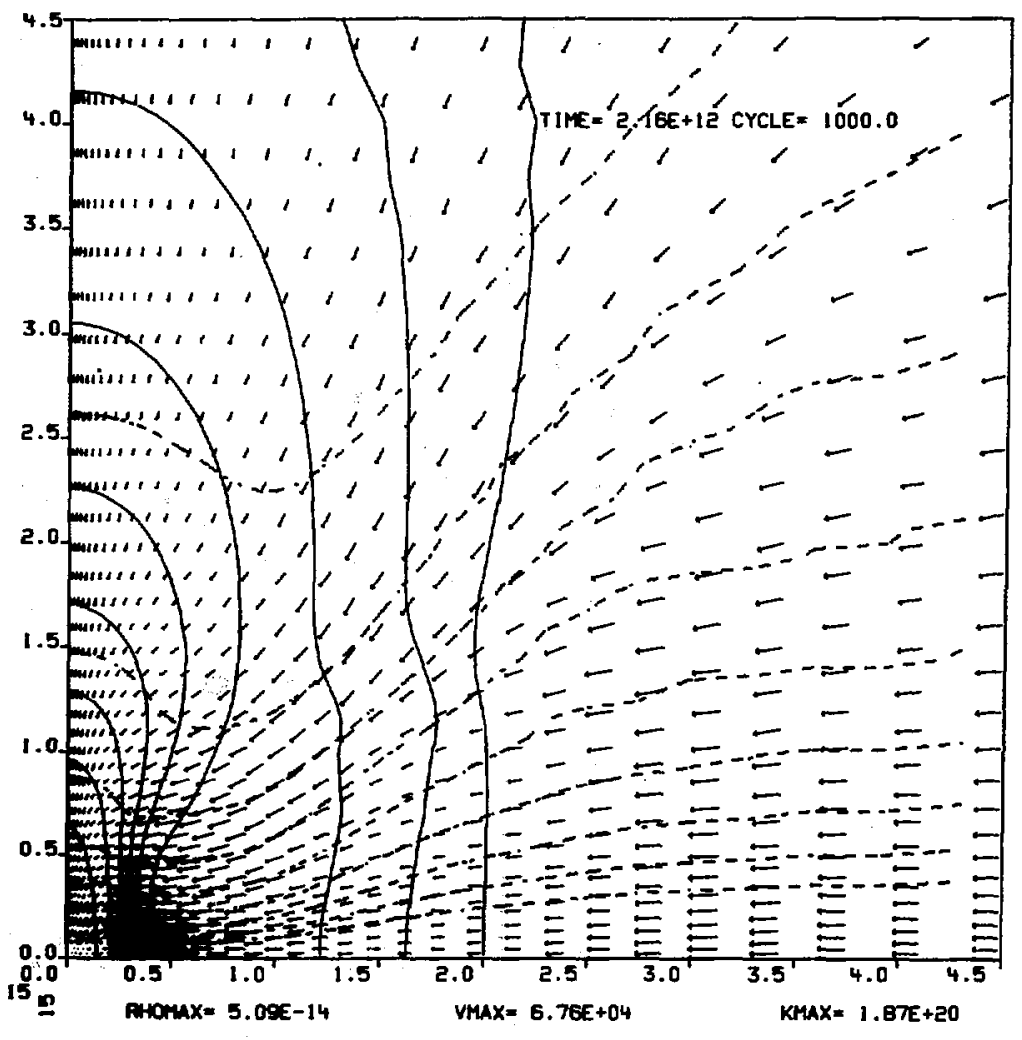

w

Dhour oldentive 21

Frame 5B.7 


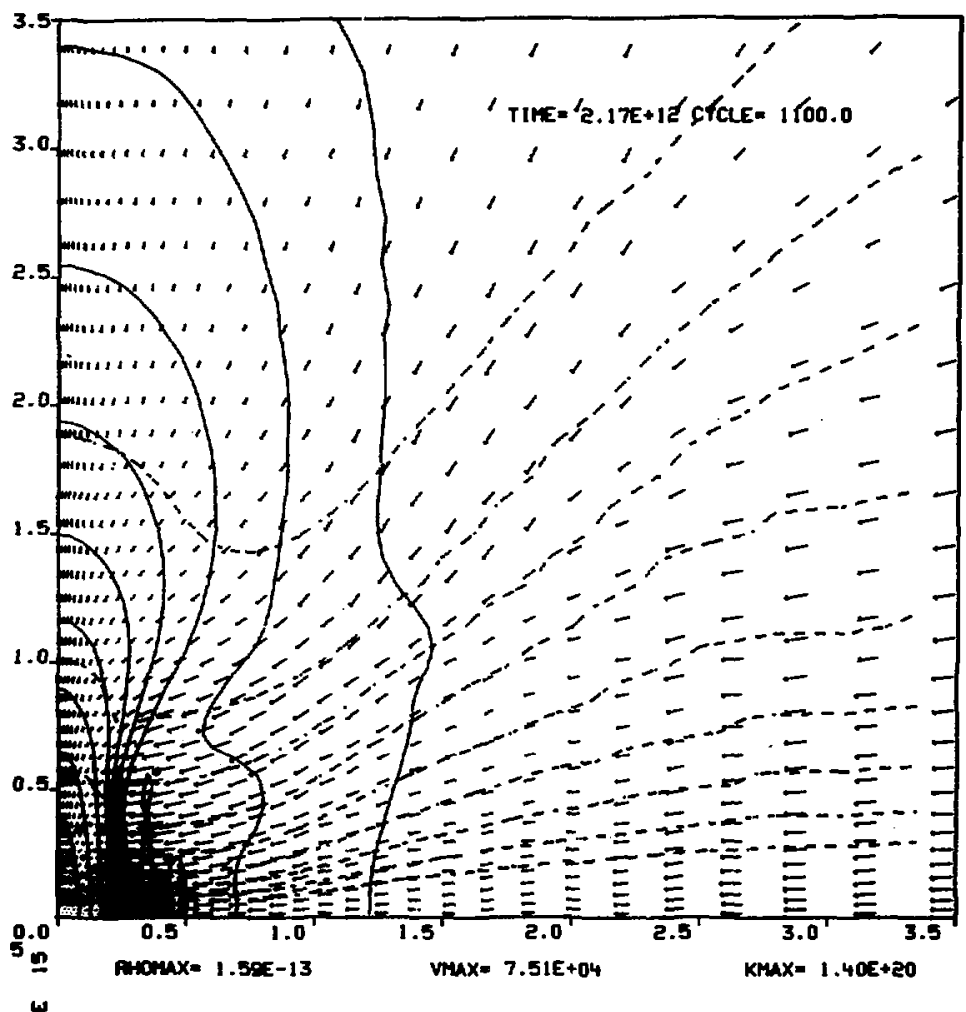

onerar mentife u

Frame 5B.8 


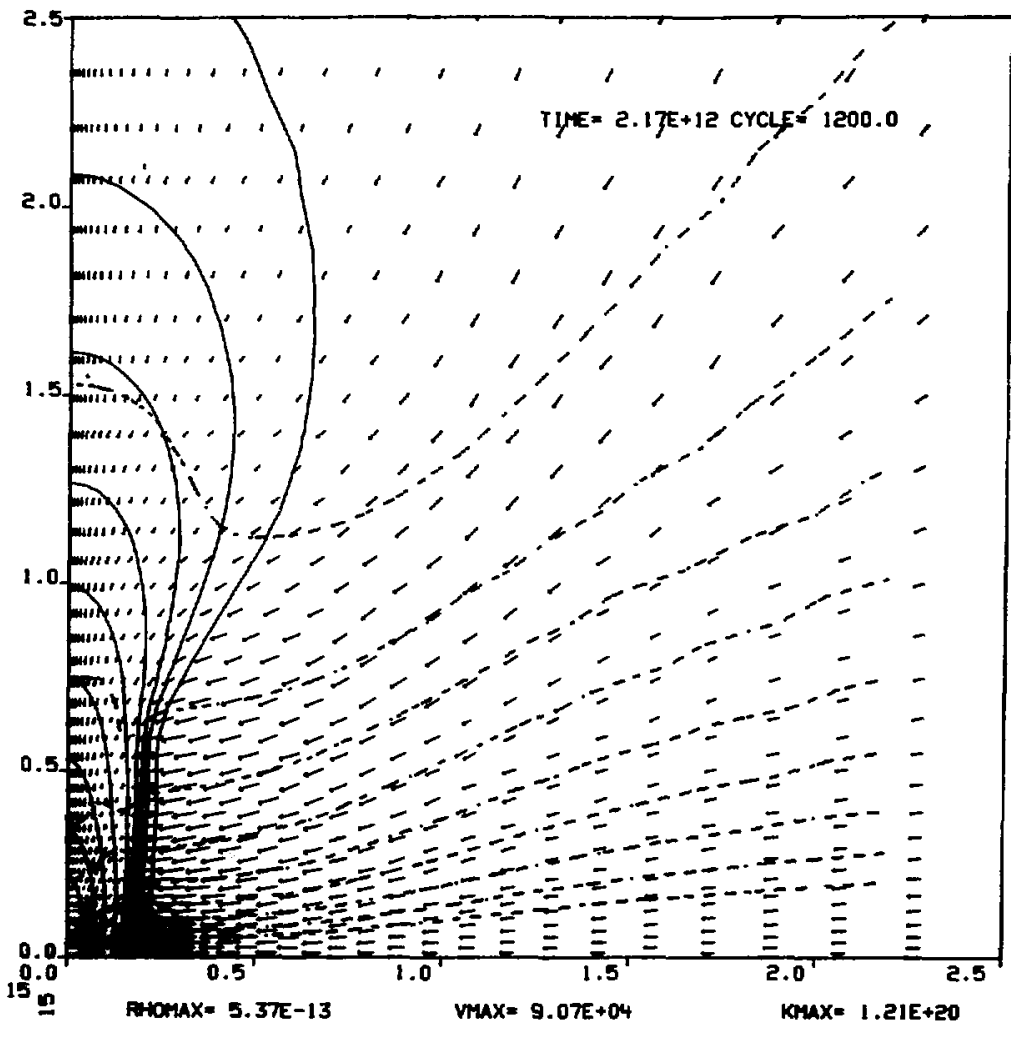

$\boldsymbol{\omega}$

Frane 5B.9 


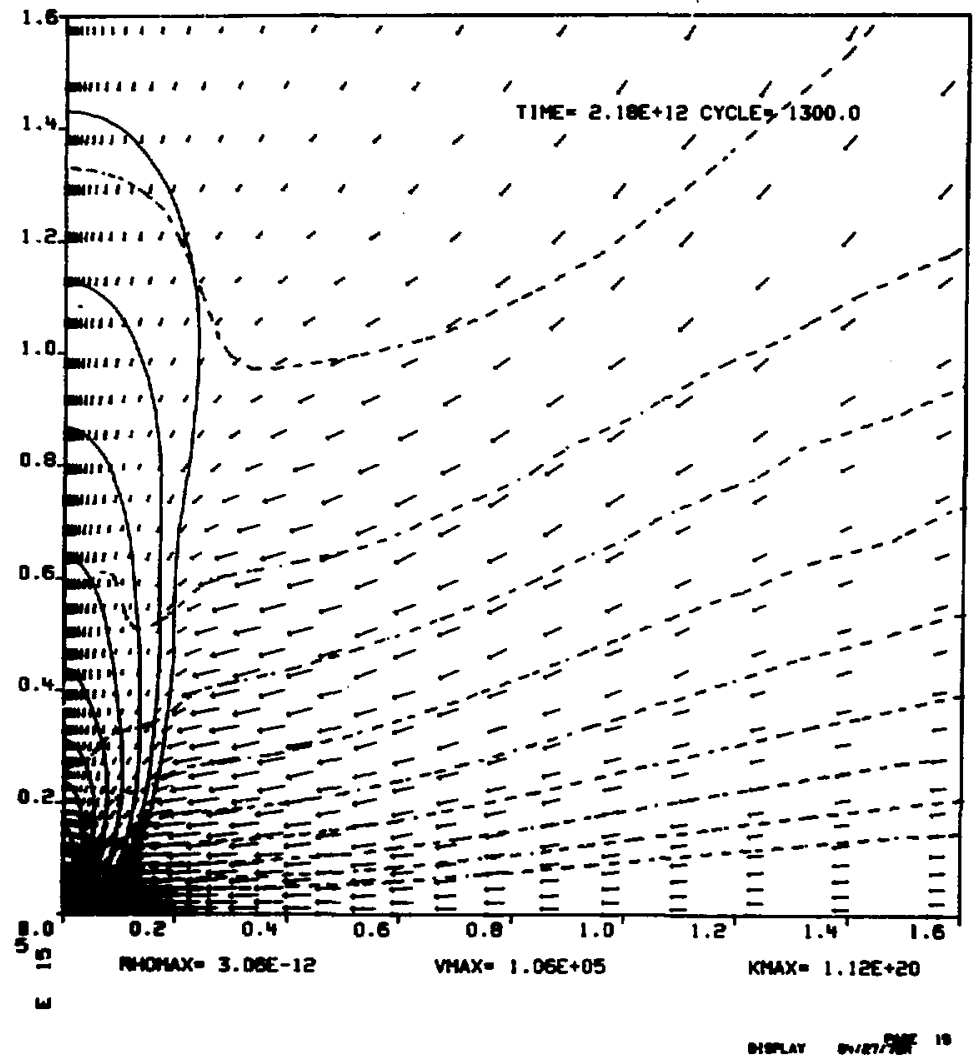

Frame 5B.10 


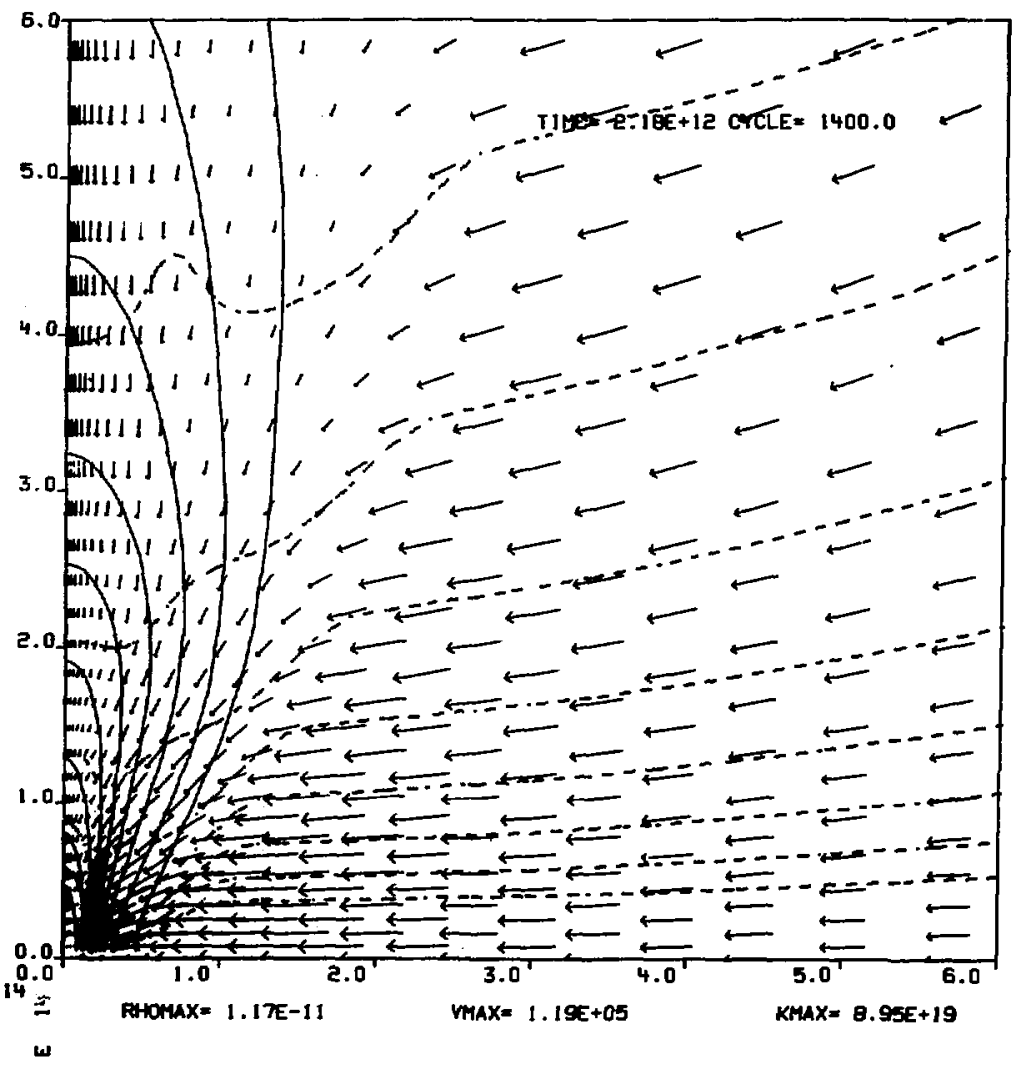

odenay movity as

Frame 5B.11 


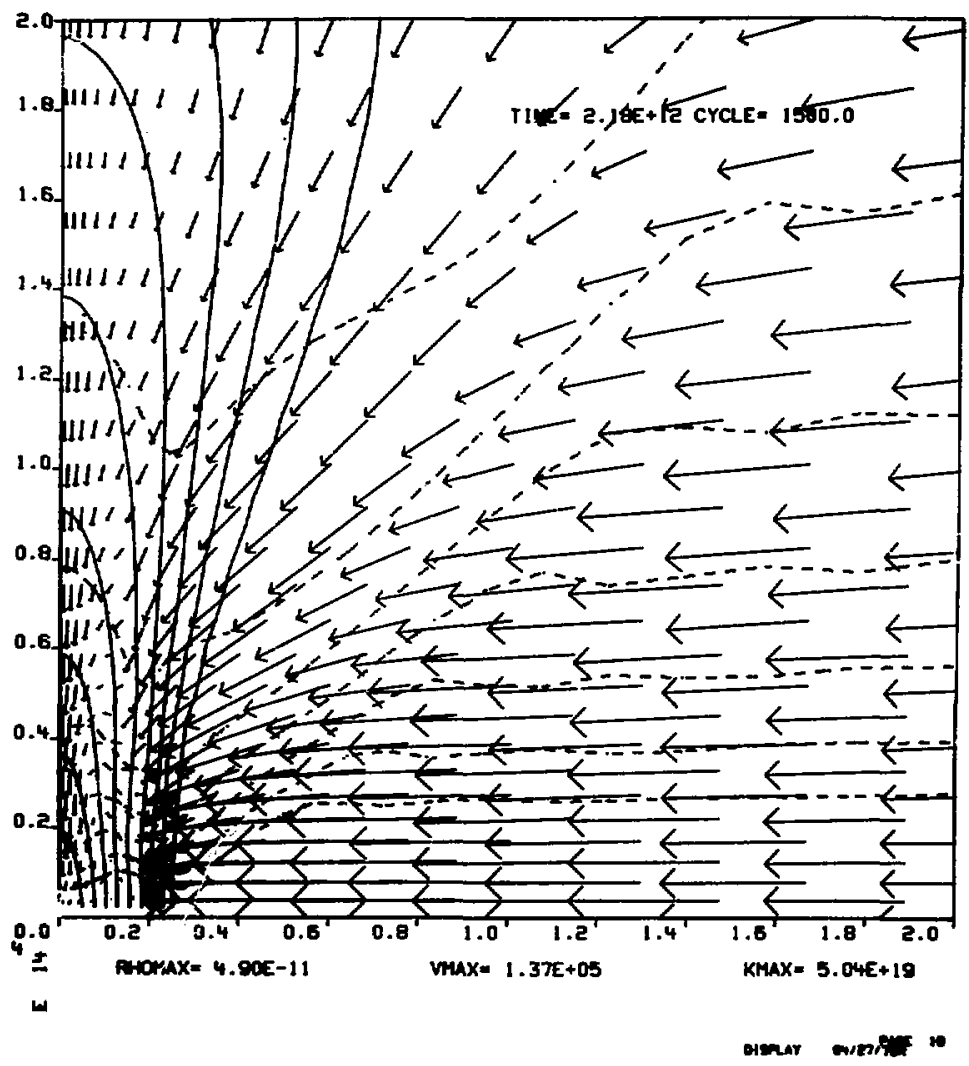

Frame 5B. 12 
Evolution 5C 


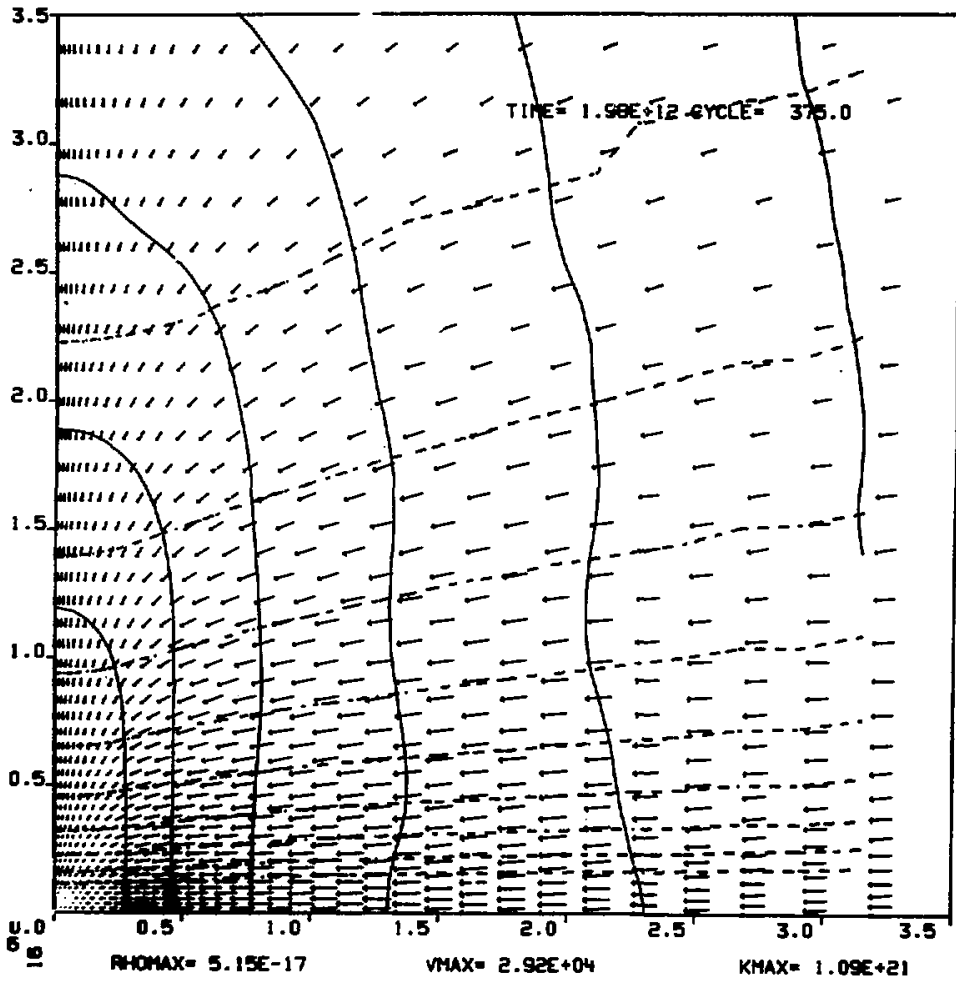

w

aiguar eirntiv?

Frame 5C.1 


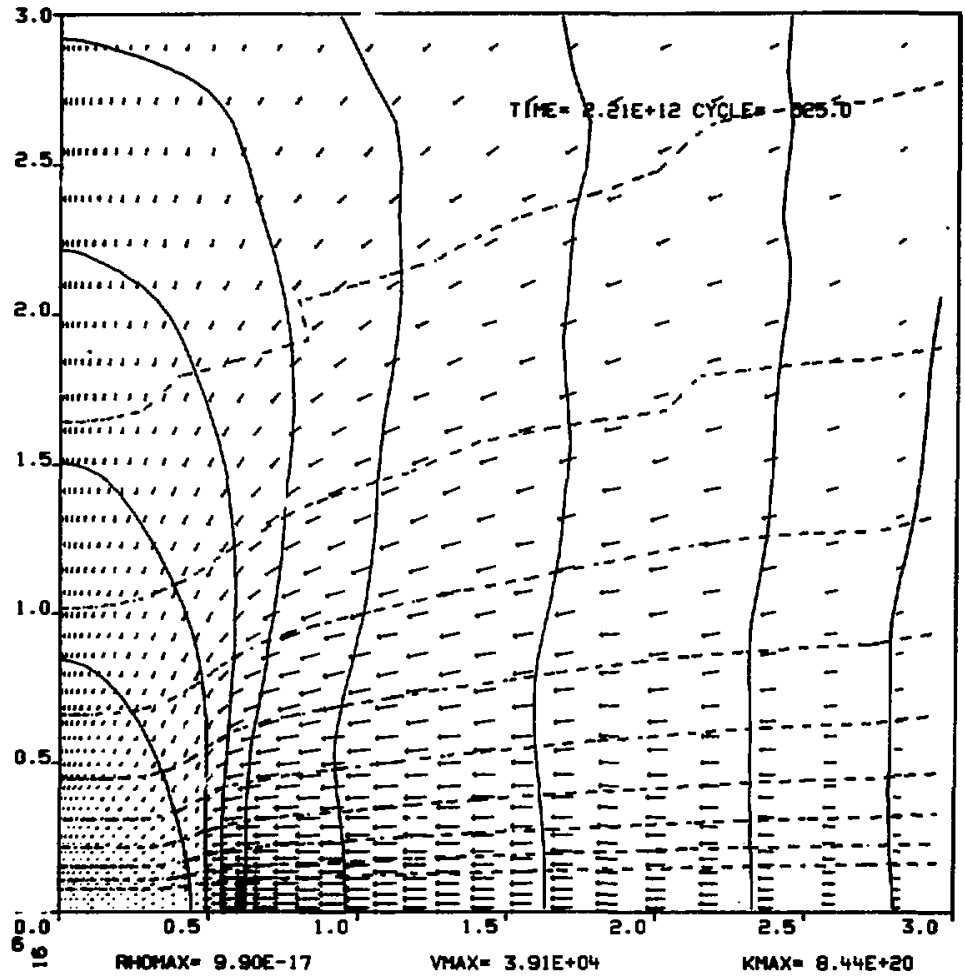

$\omega$

denur montia

Frame 5C.2 


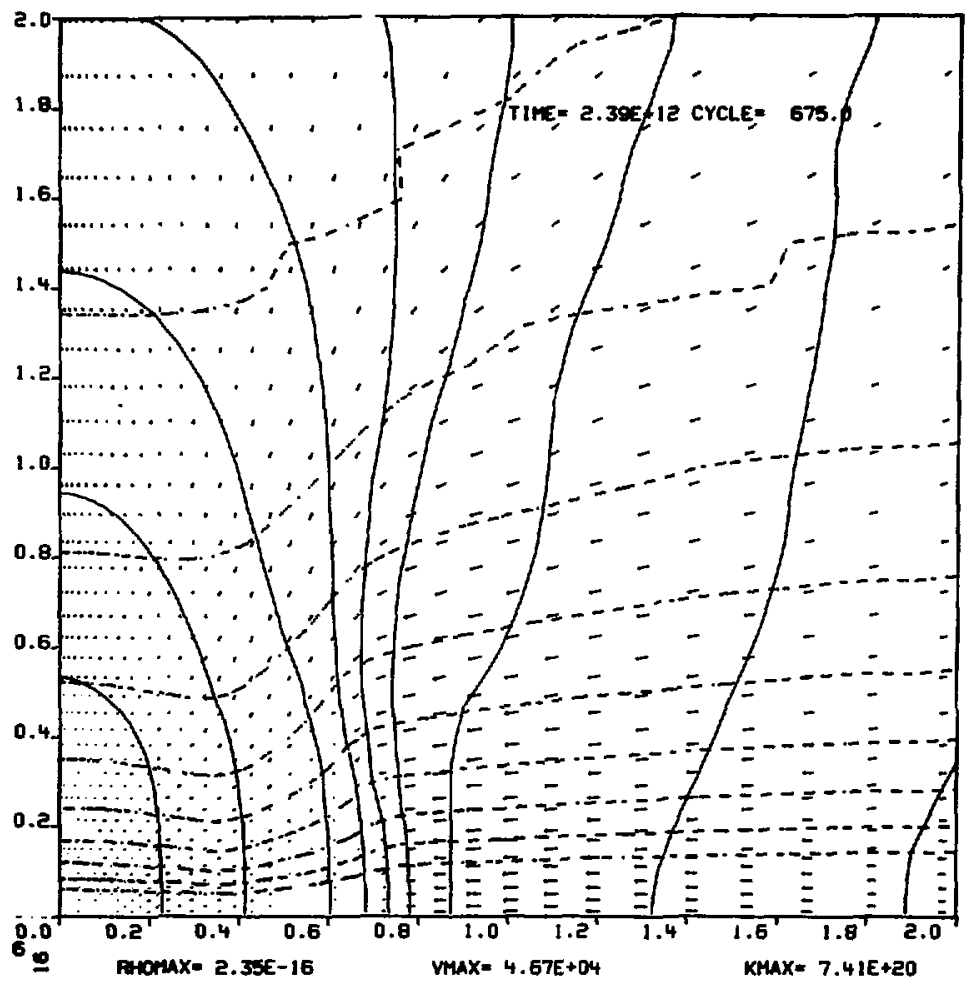

$\boldsymbol{\omega}$

olsur mentip ie

Frame $5 \mathrm{C} .3$ 


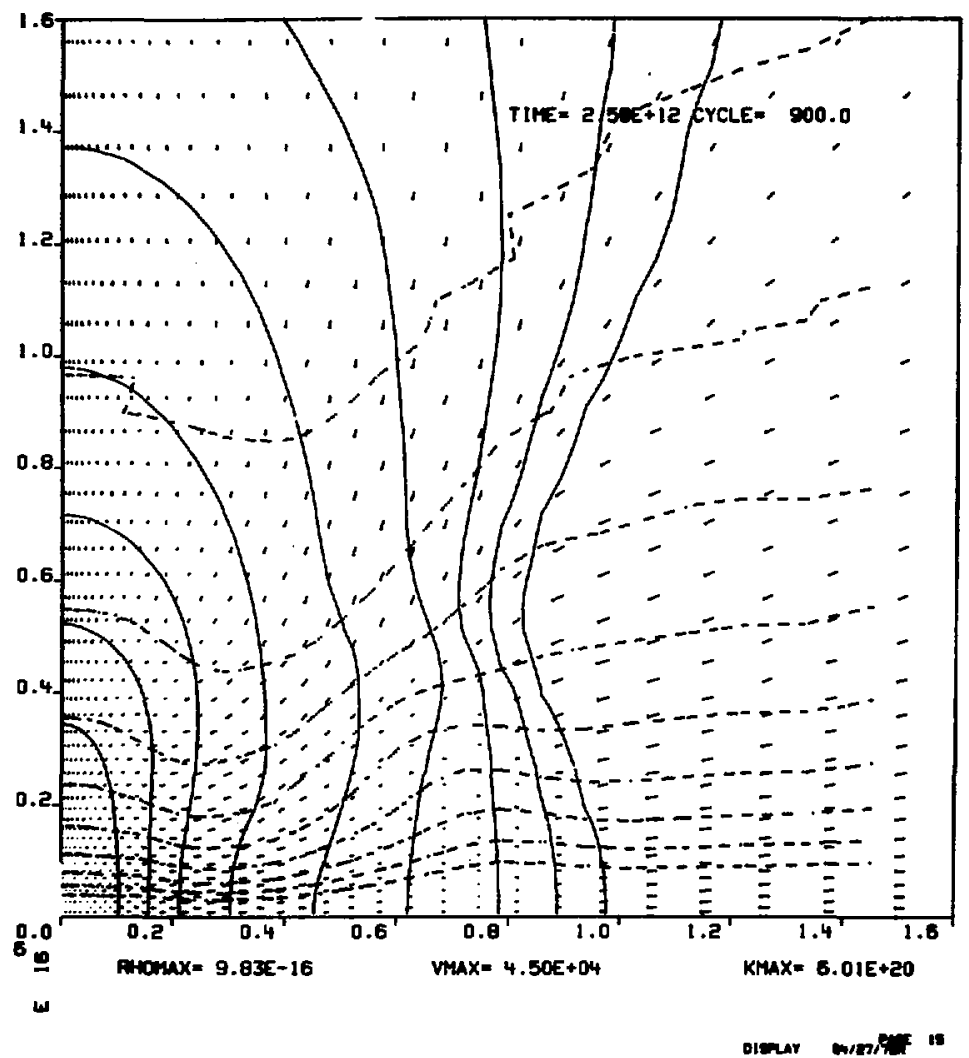

Frame $5 \mathrm{C} .4$ 


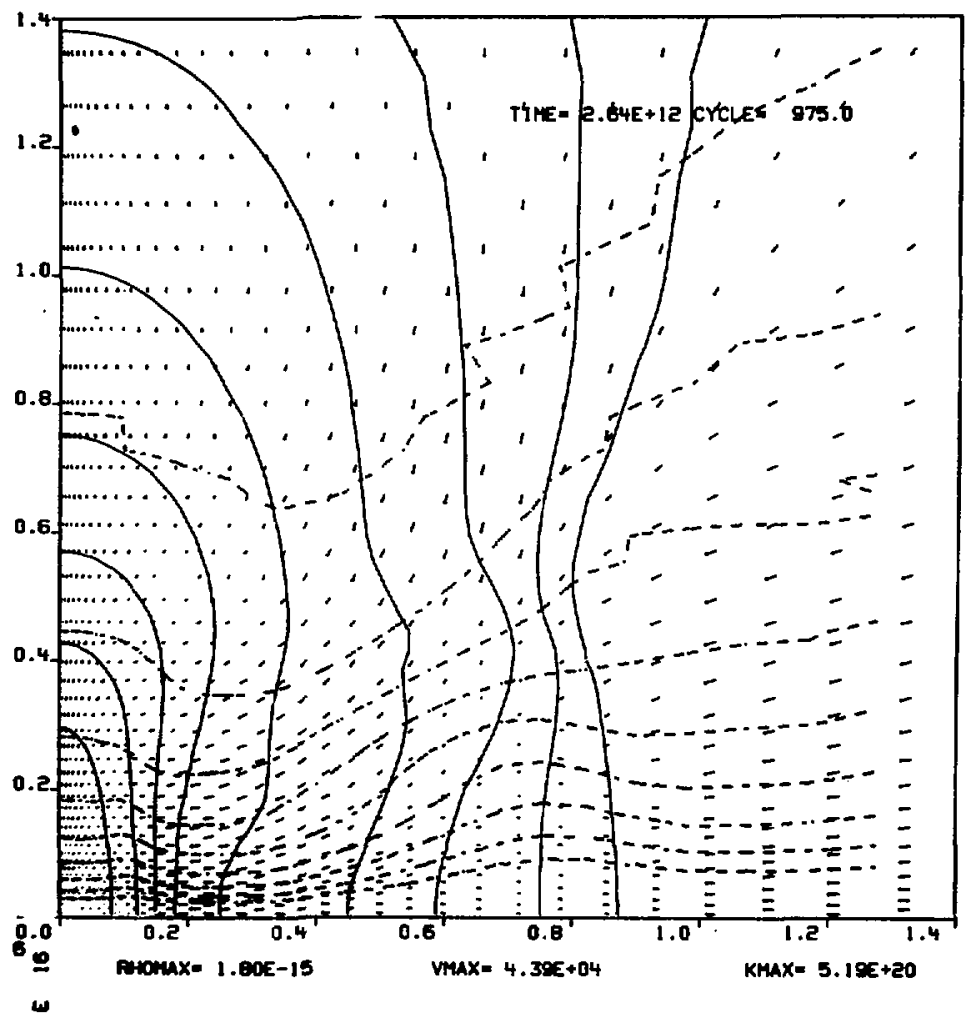

oterar muntive is

Frane 5C.5 


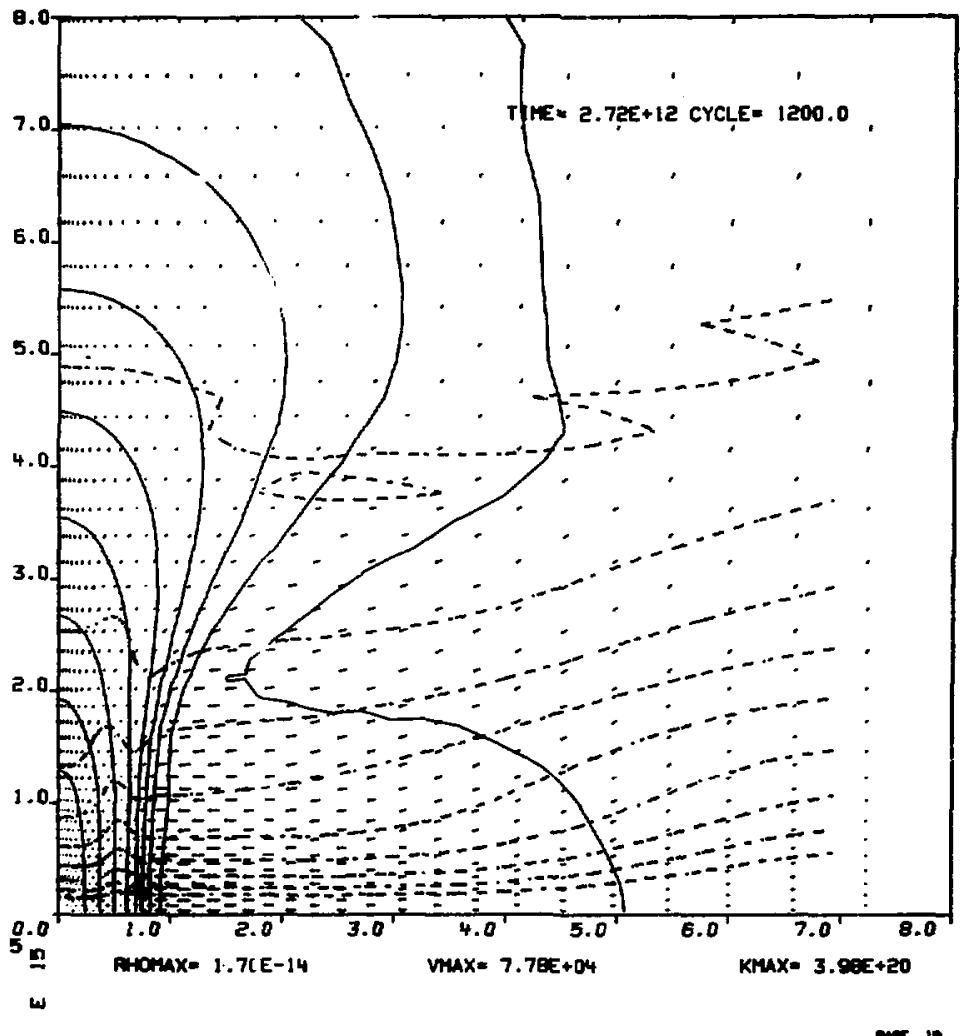

ouray muentive to

Frame 5C.6 


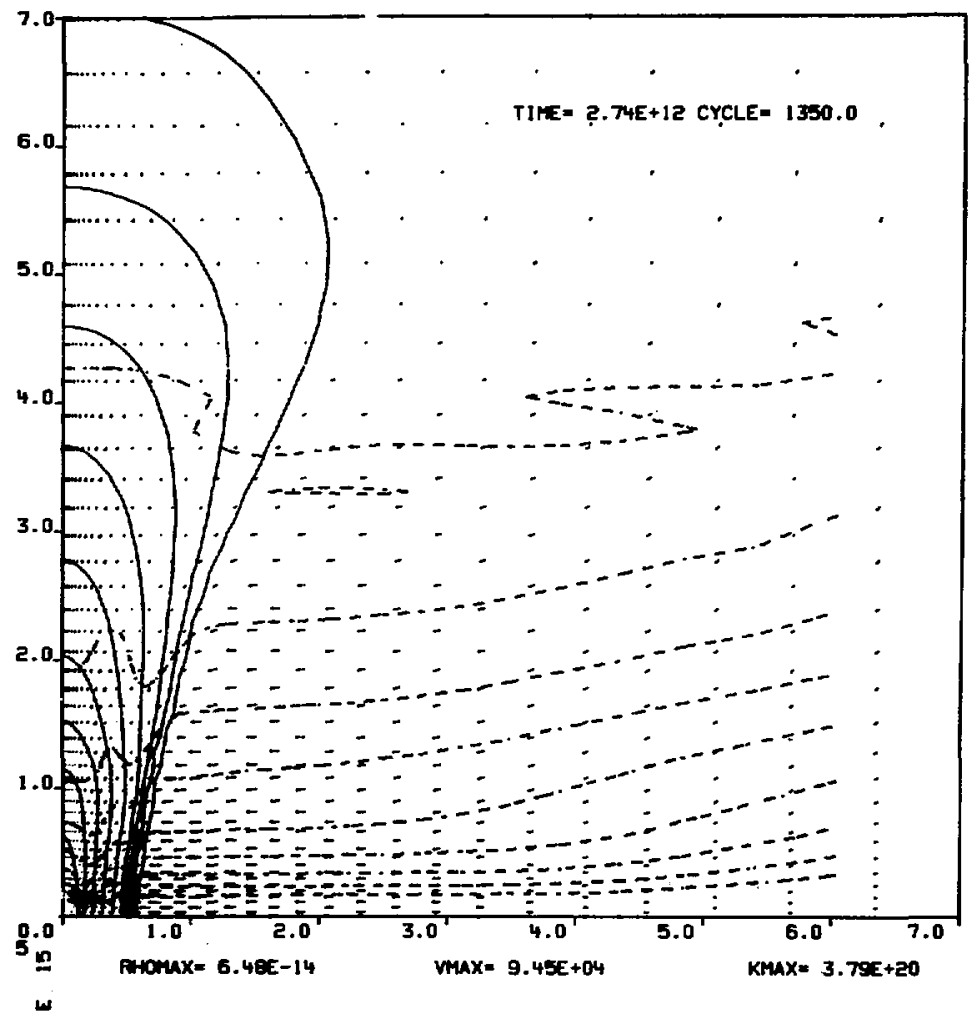

Dienar moerfice a

Frame $5 C .7$ 


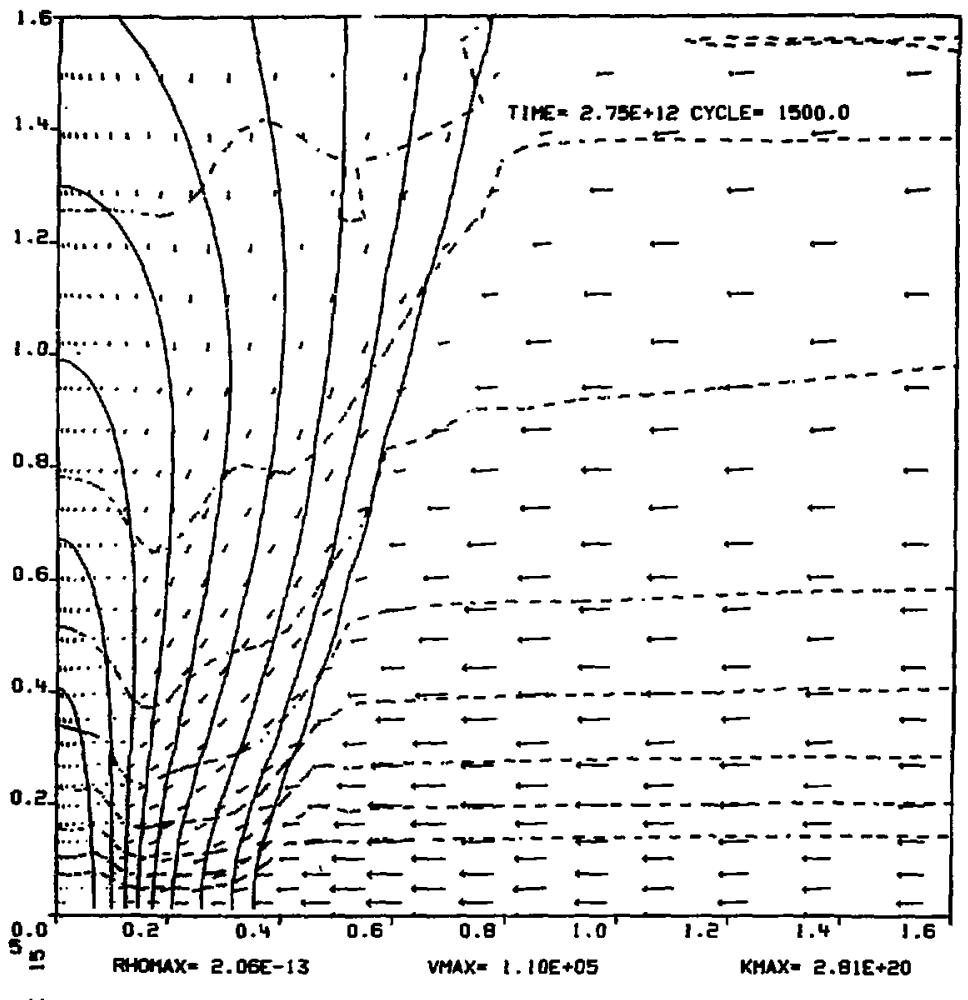

w

Frame $5 C .8$ 
143

Evolution 5D 


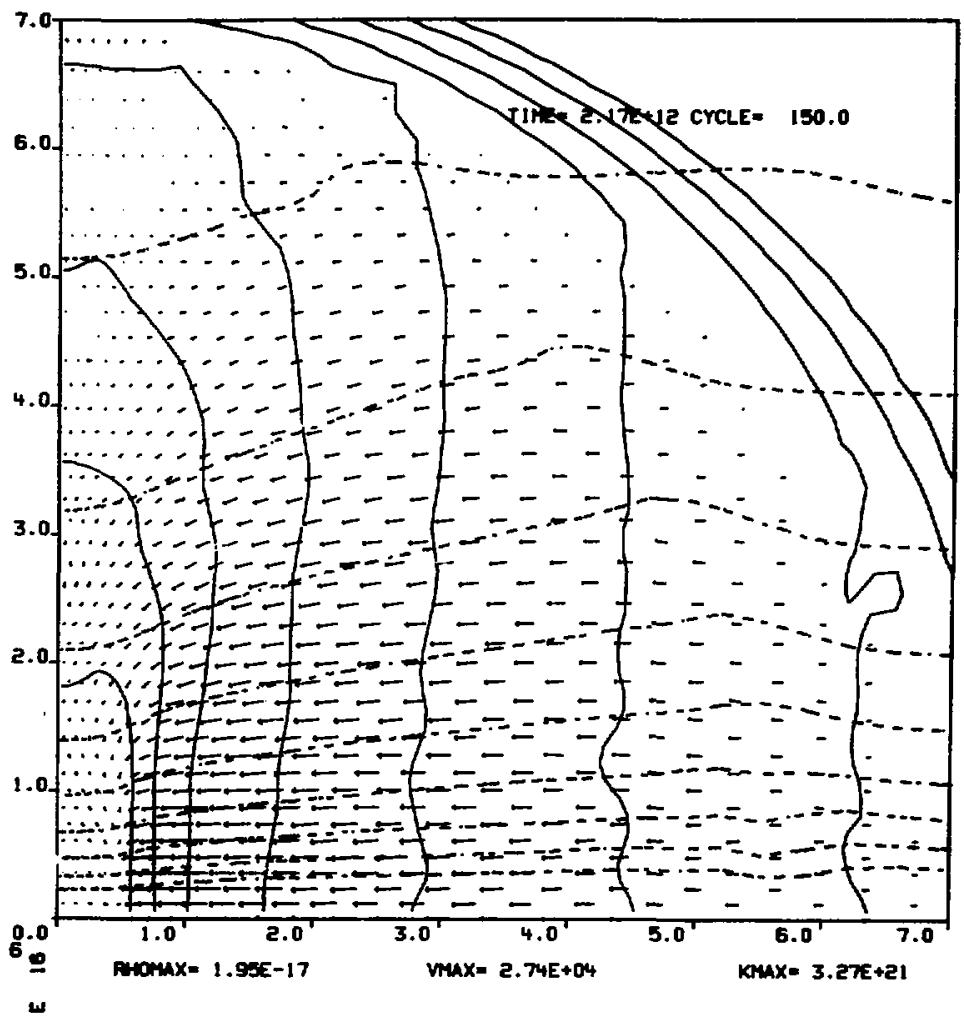

cuerar enenting

Frane 5D.I 


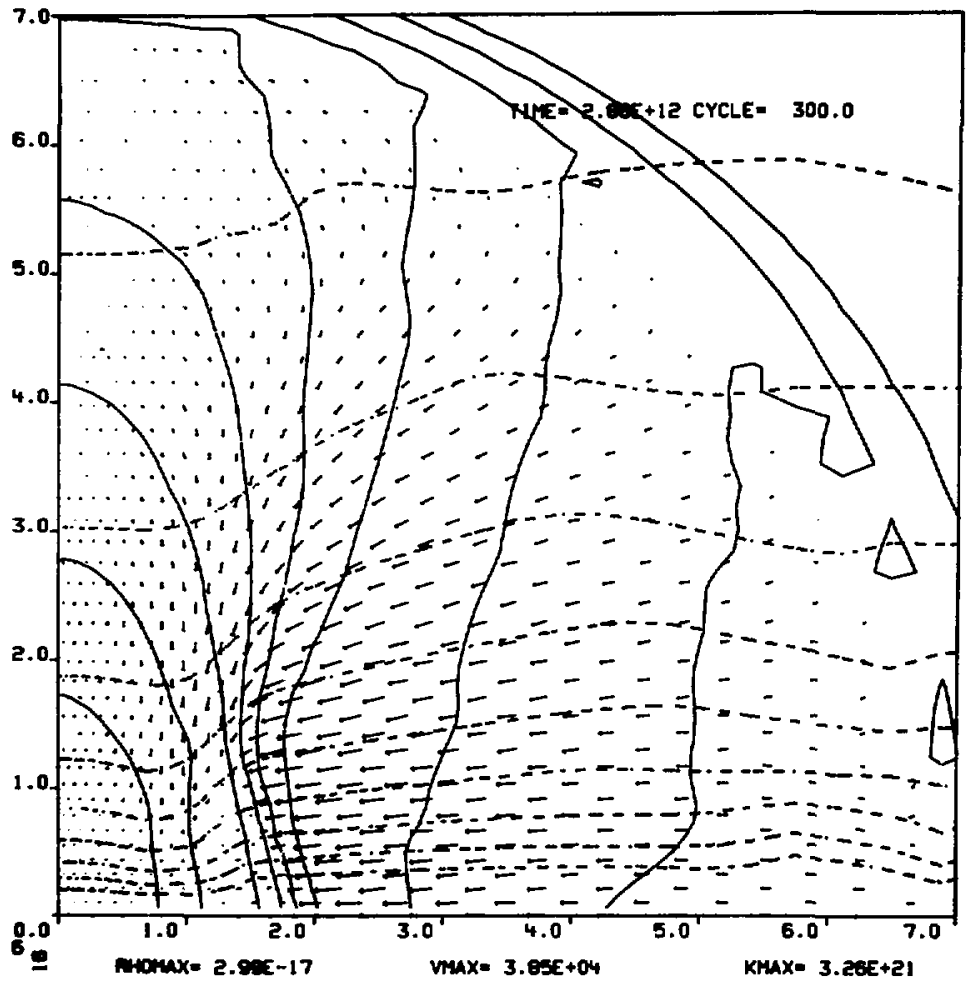

$\omega$

dereur meritia

Frame 5D.2 


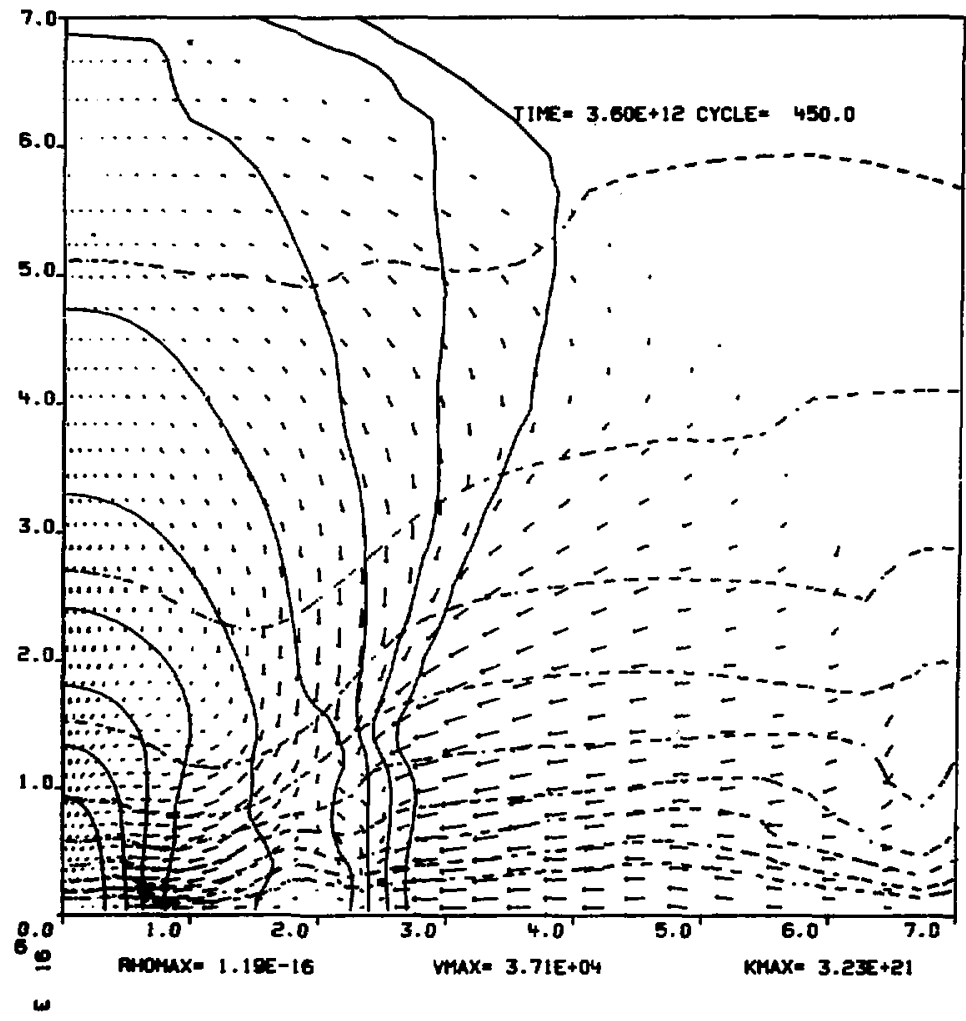

otemar mueptits

Frame 5D.3 


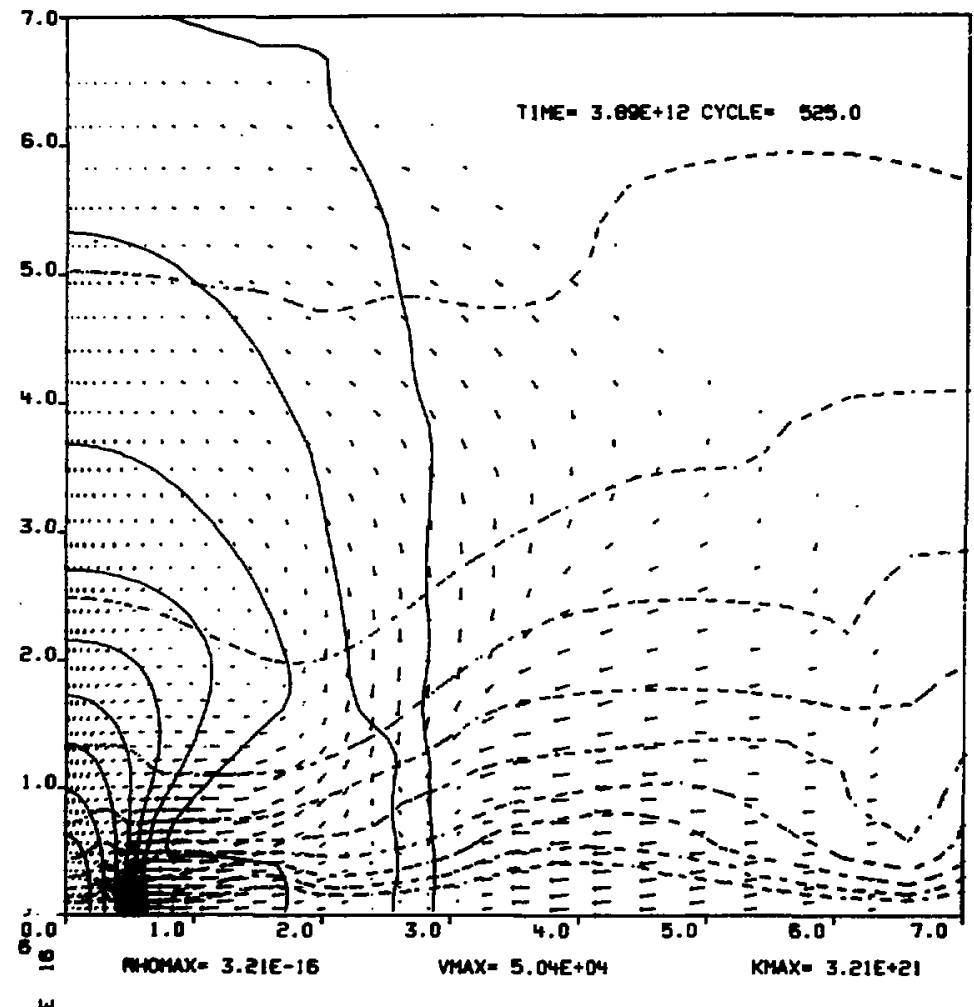

menar mentip.

Frame 5D.4 


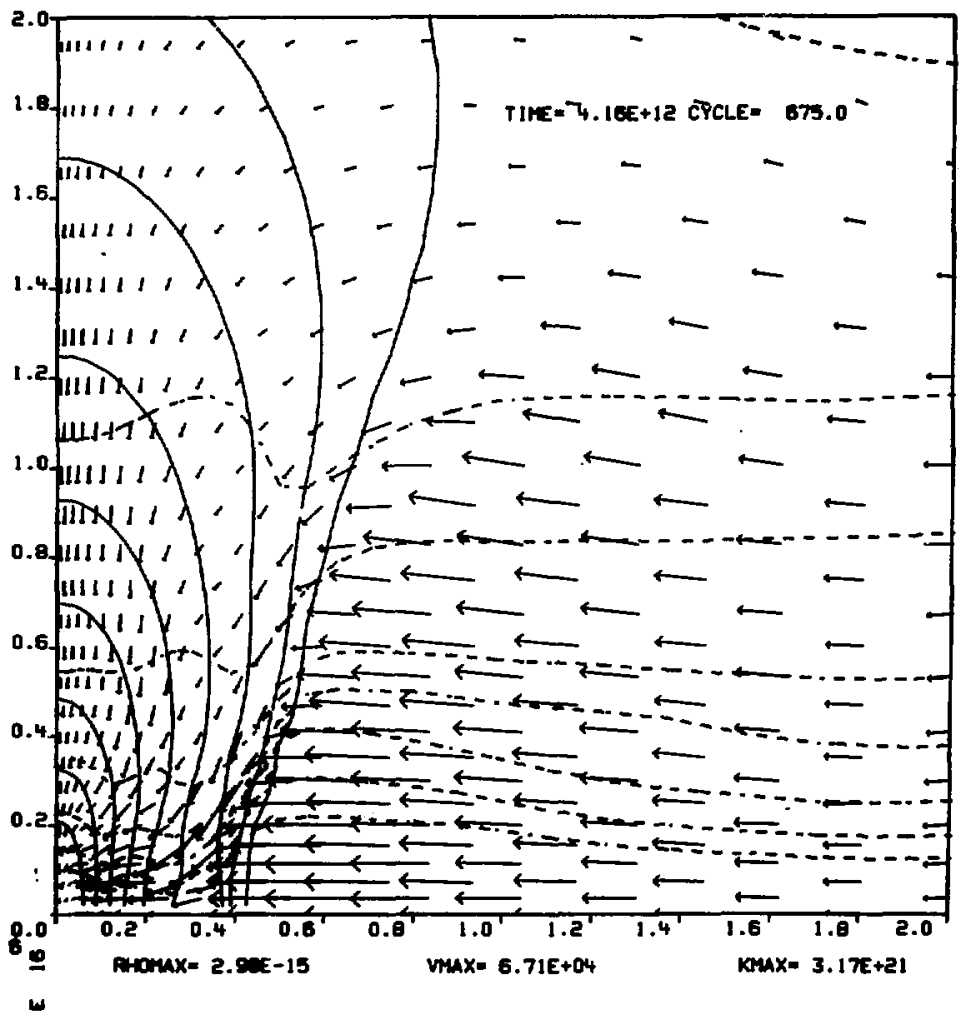

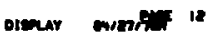

Frame 5D.5 


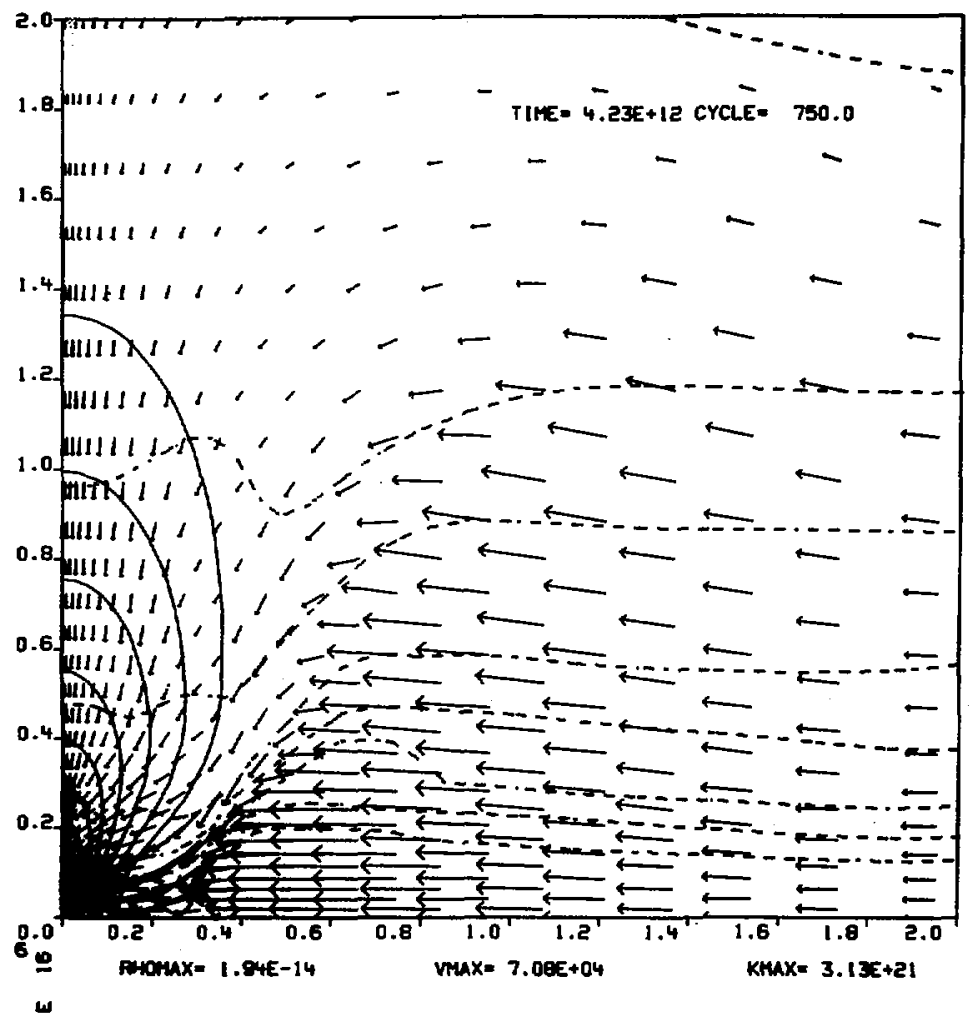

derear munfite is

Frame 5D. 6 


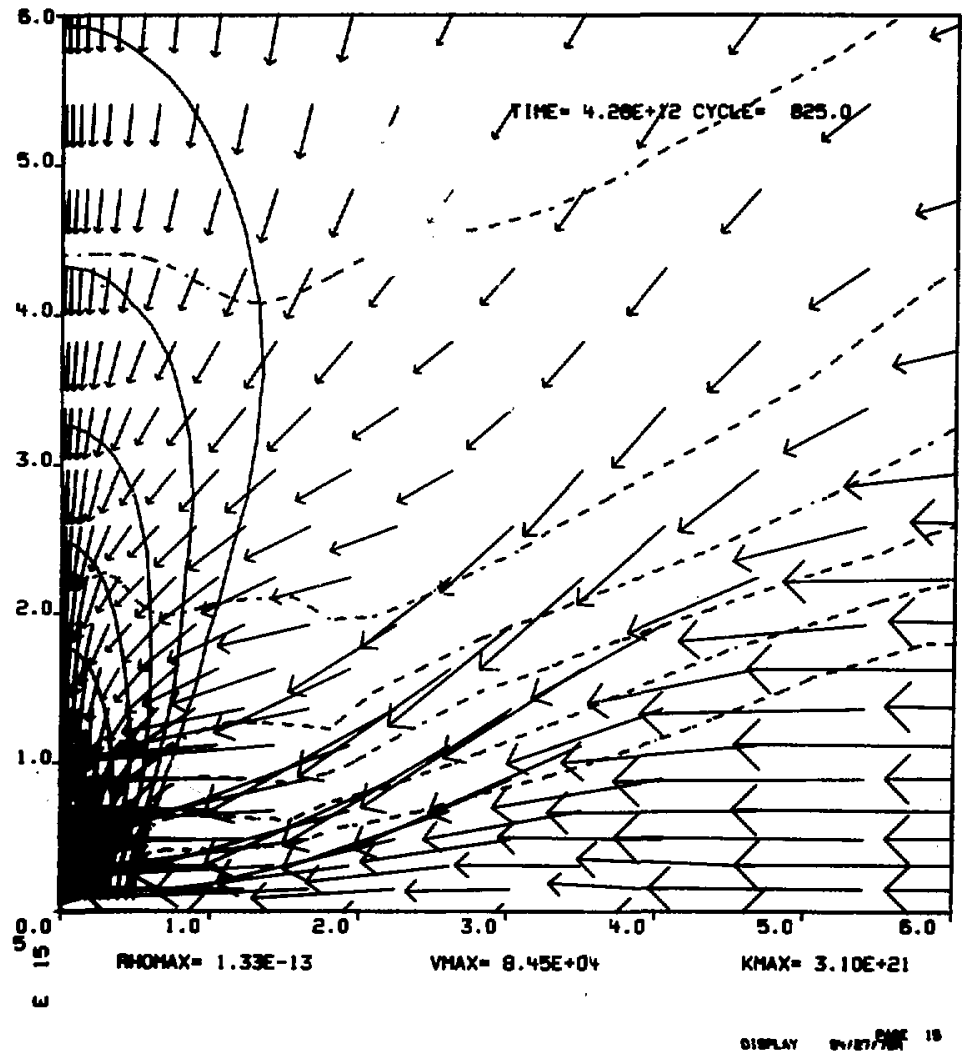

Frame 5D.7 
Evolution $5 E$ 


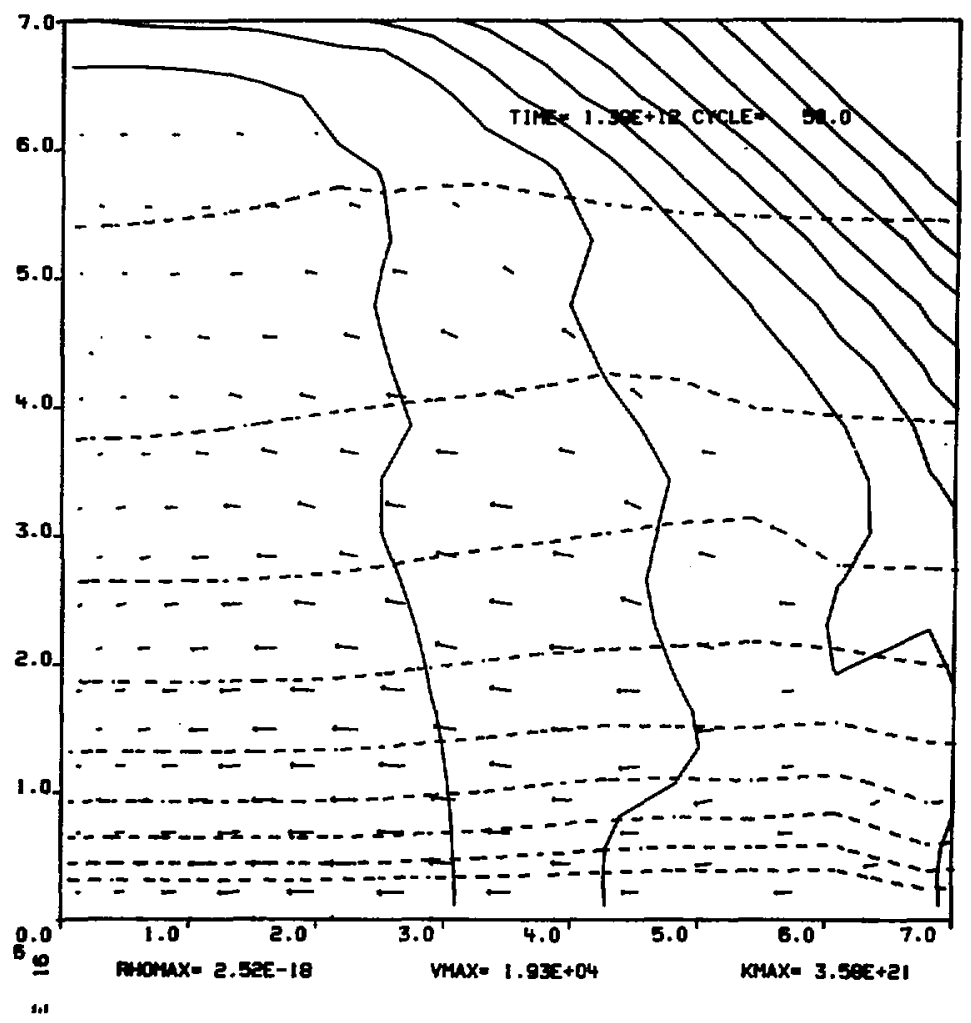

Frame 5E. 1 


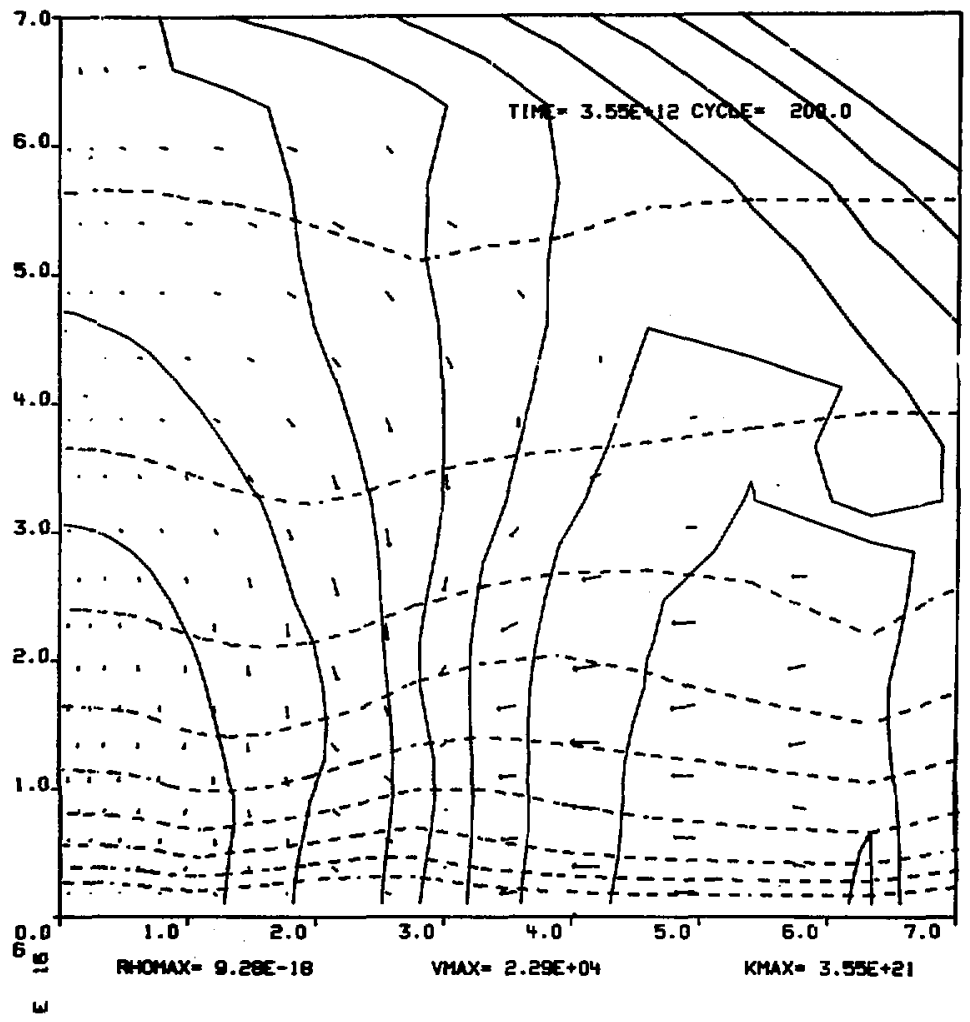

dumar menitip

Frame 5E.2 


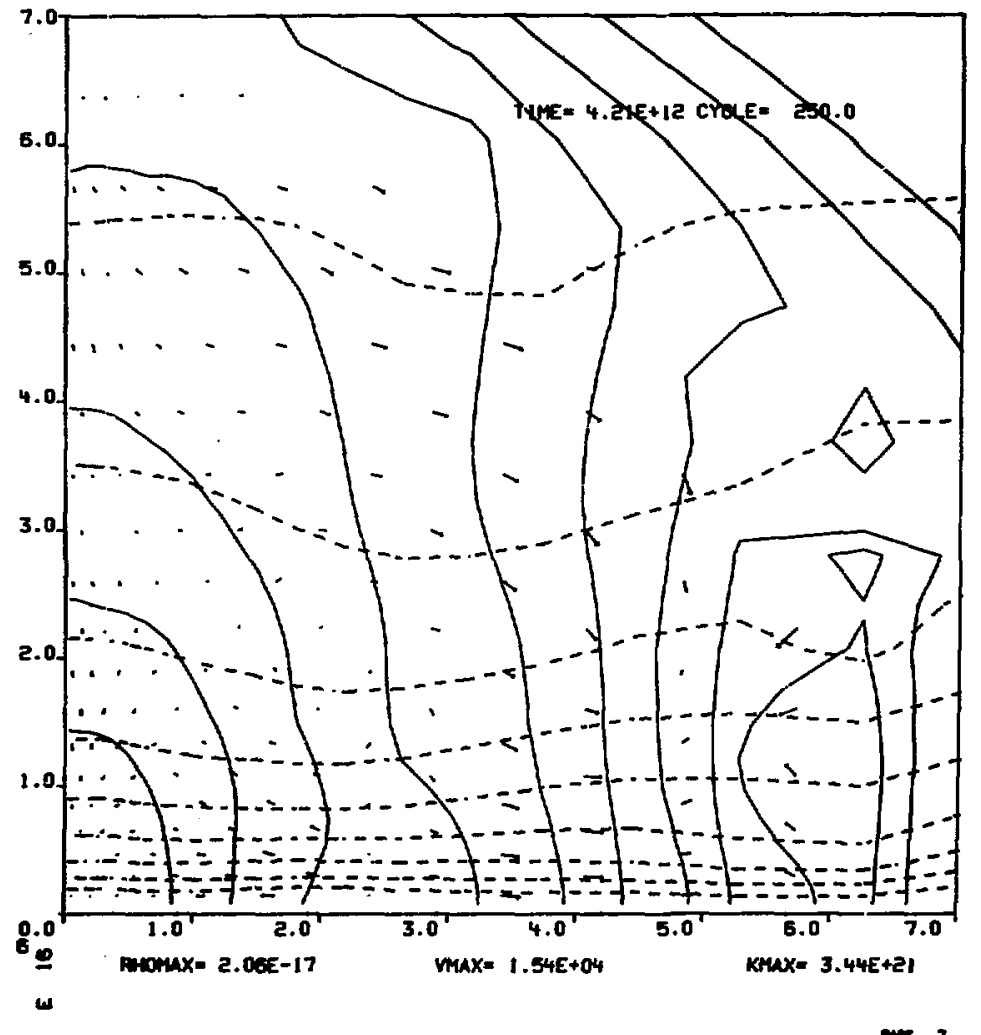

ormar whetite

Frame 5E.3 


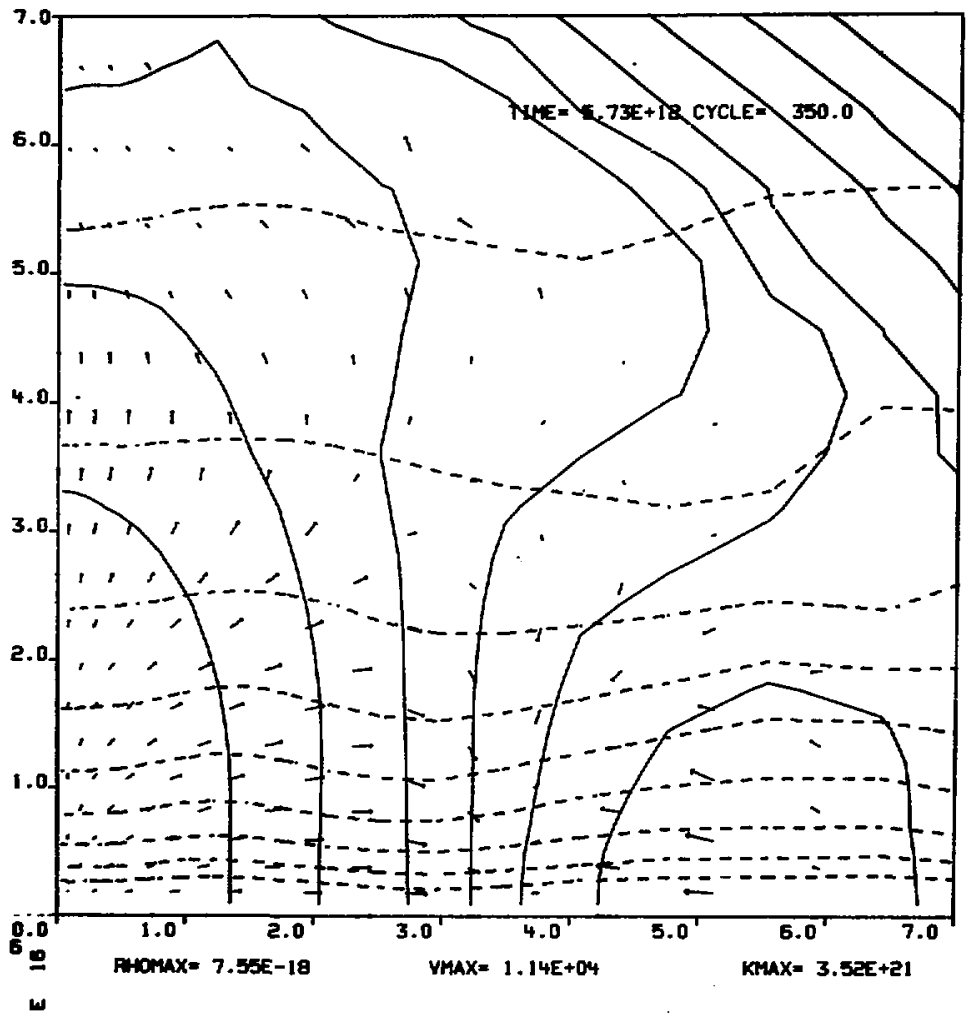

ourar montios.

Frame 5E.4 


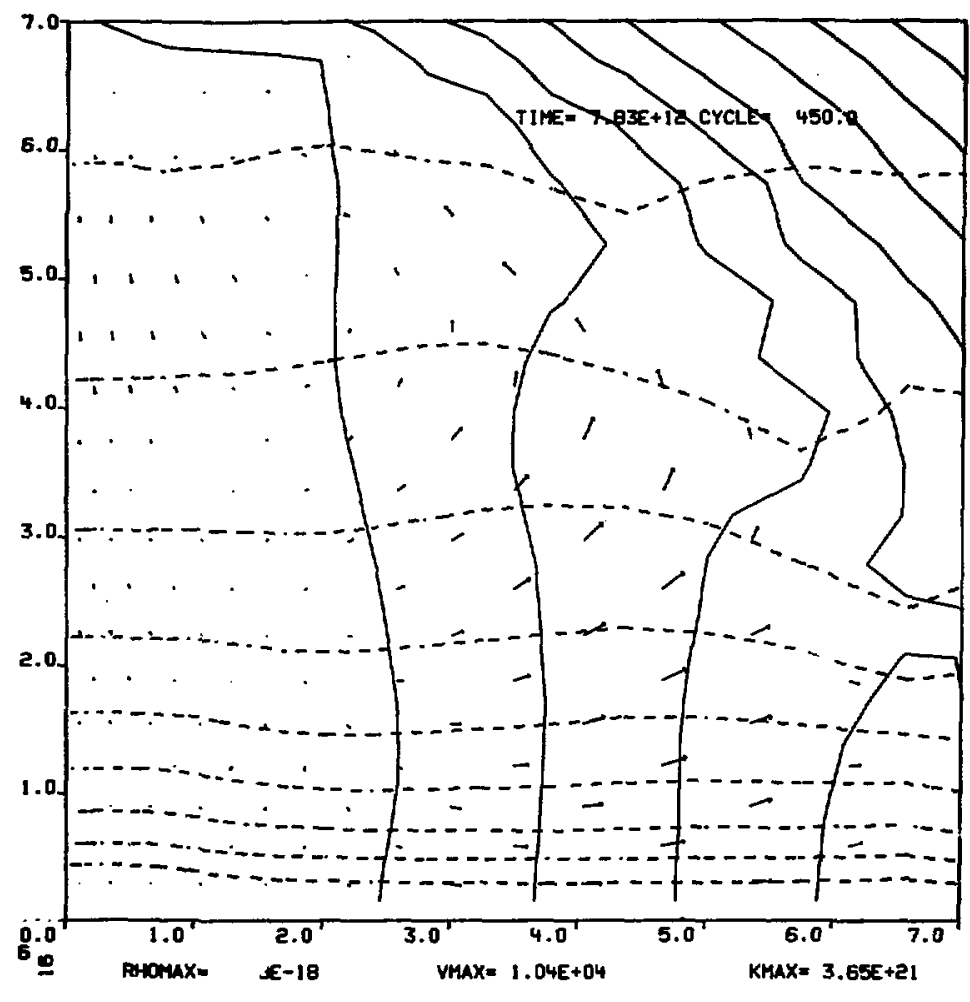

w

dimar mining

Frame 5E.5 


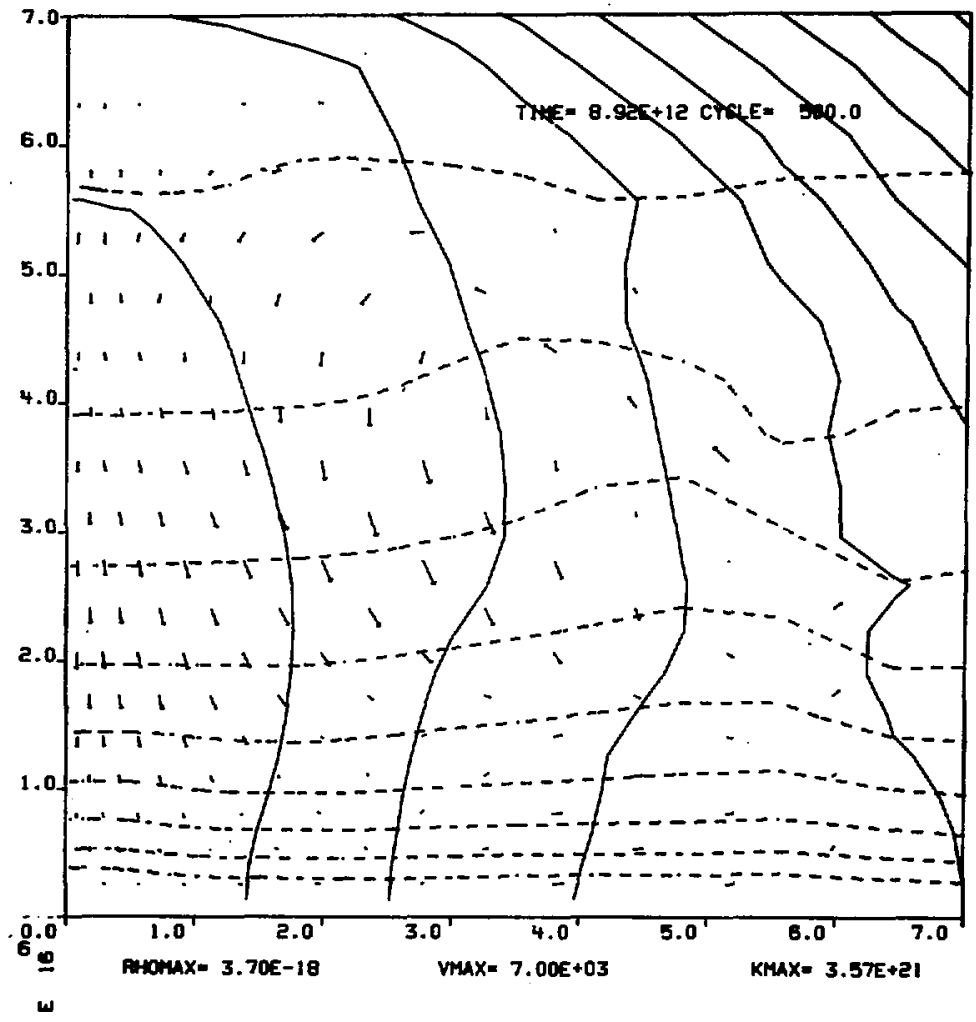

olezar eneritio le

Frame 5E. 6 


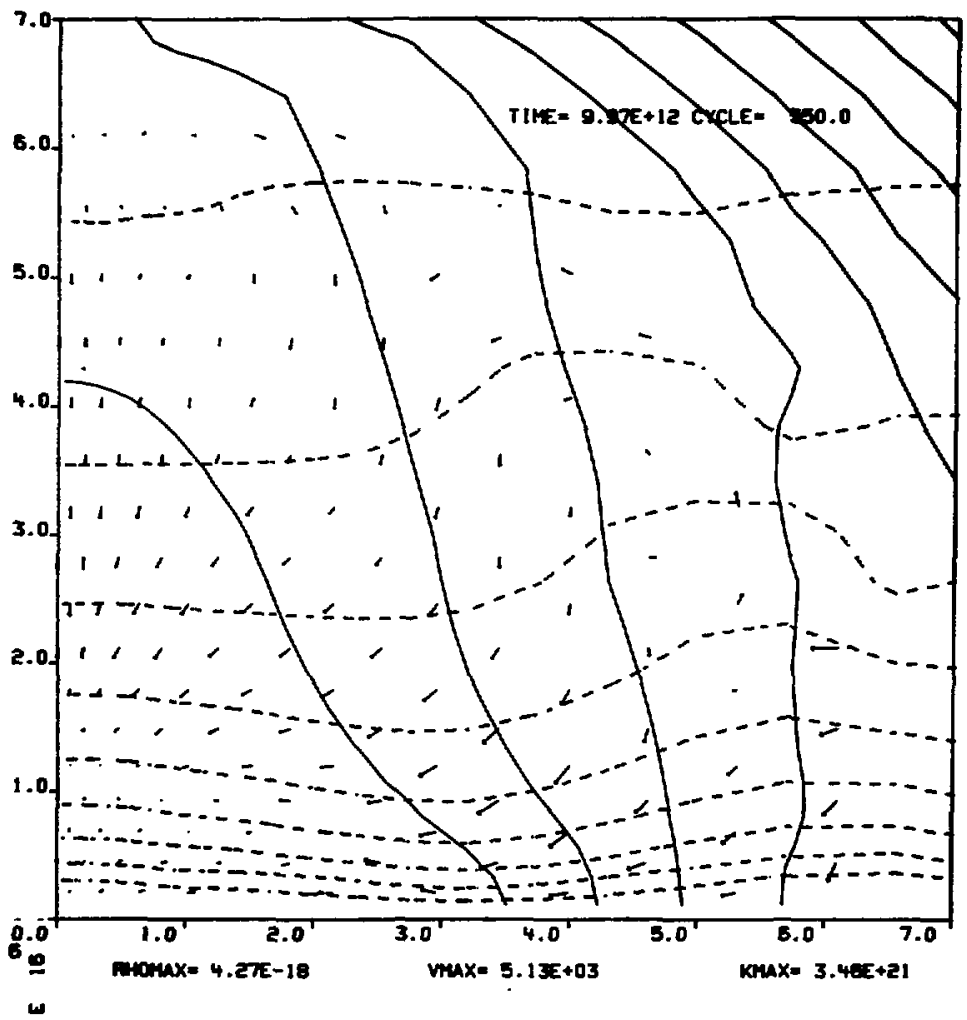

errar menfer is

Frame 5E.7 


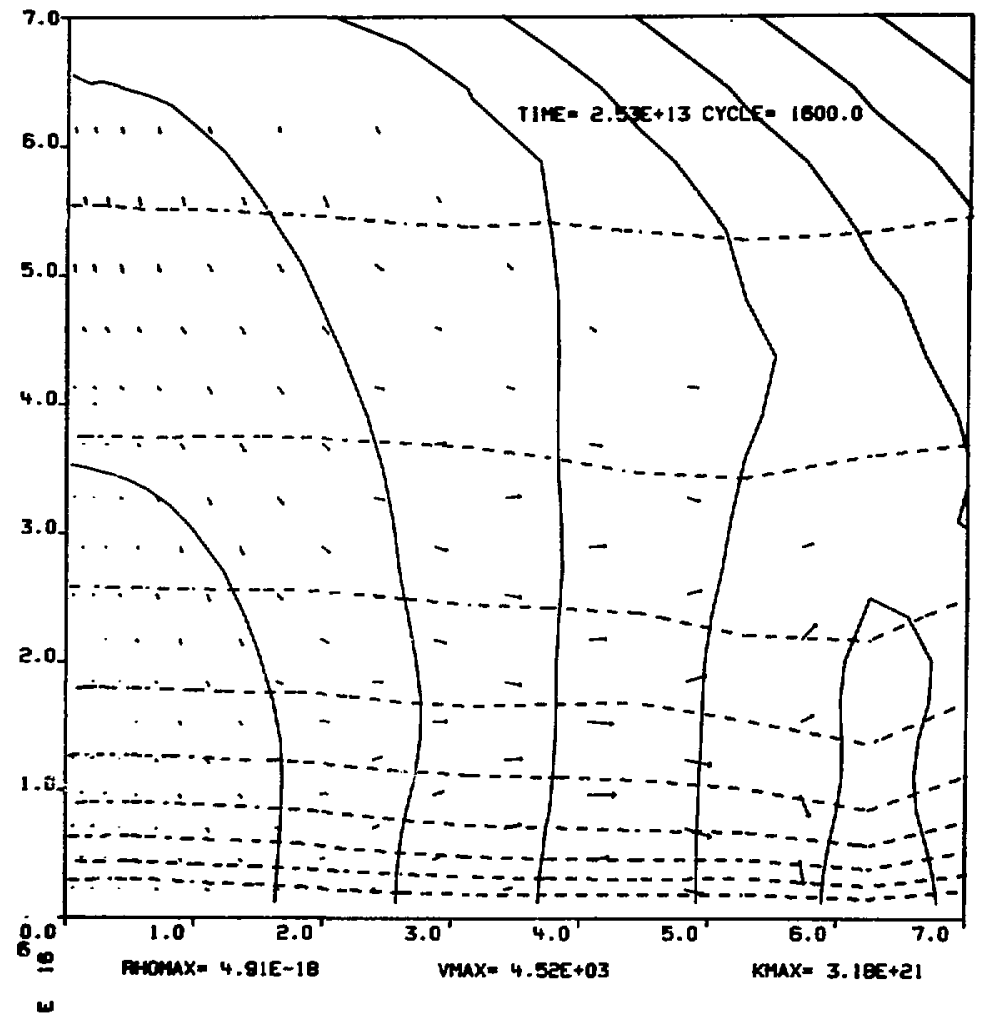

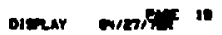

Frame 5E. 8 
Givolution 5E' 


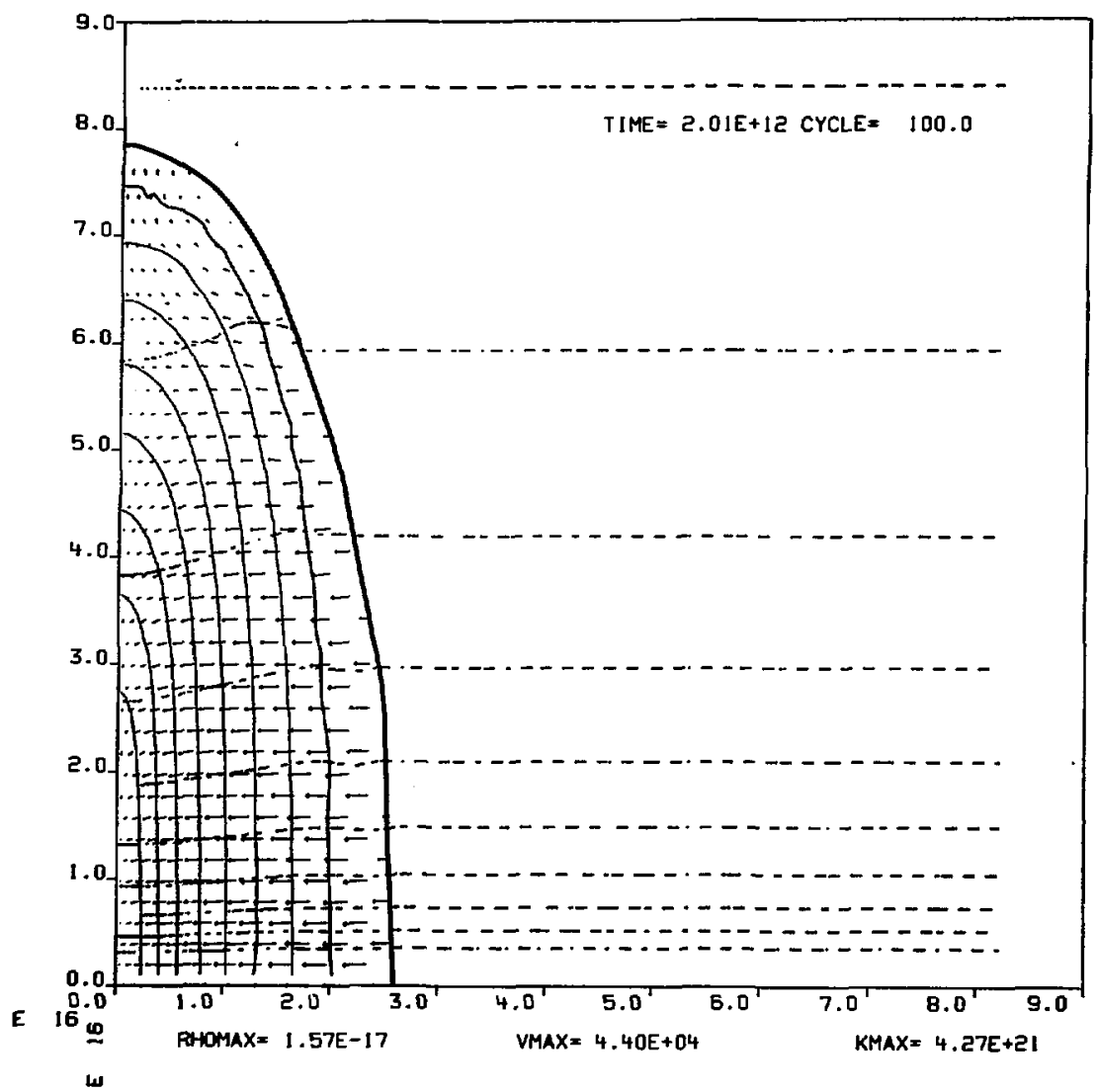

orsmar ourentipe 3

Frame 5E'.1 


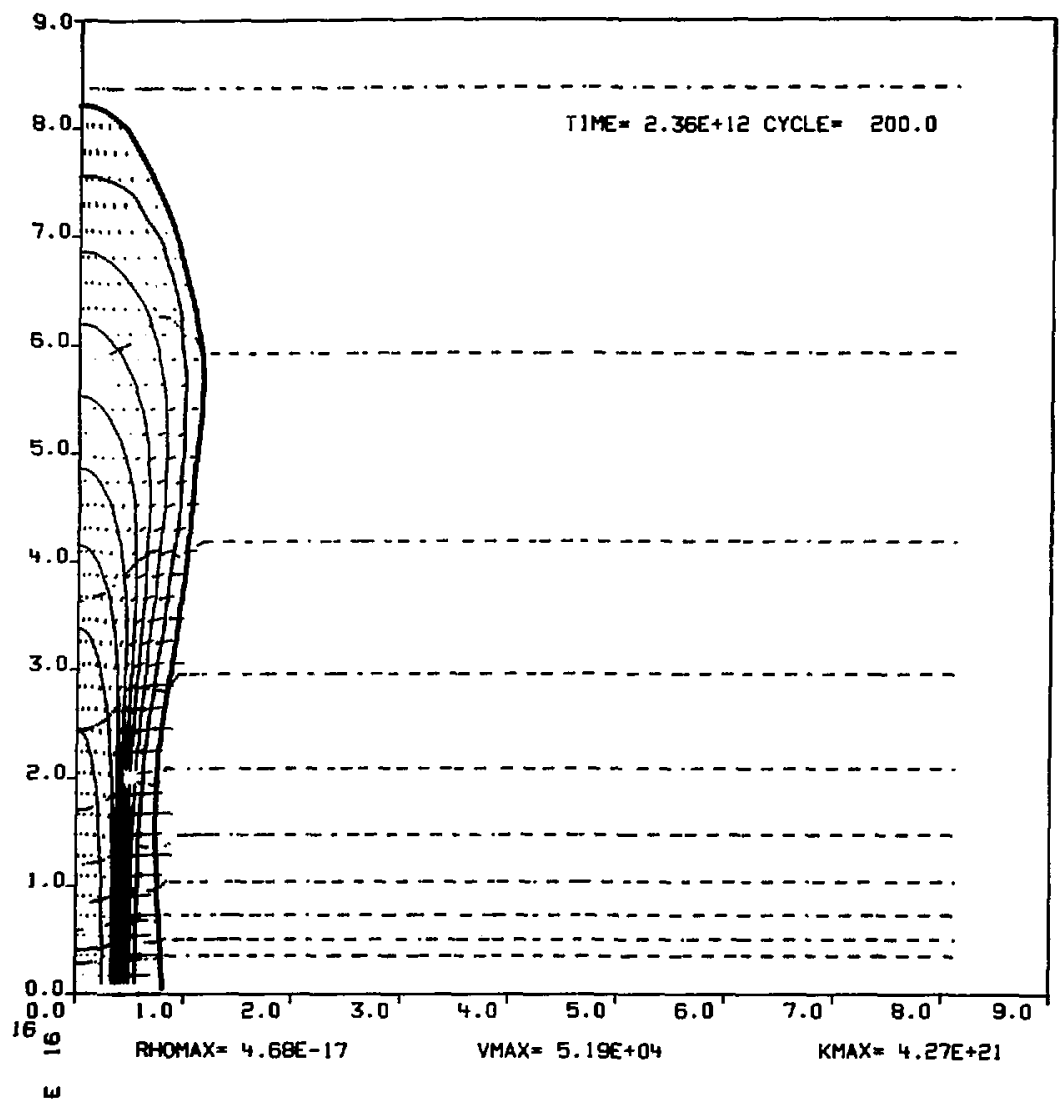

oismar onezitive

Frame 5E'.2 


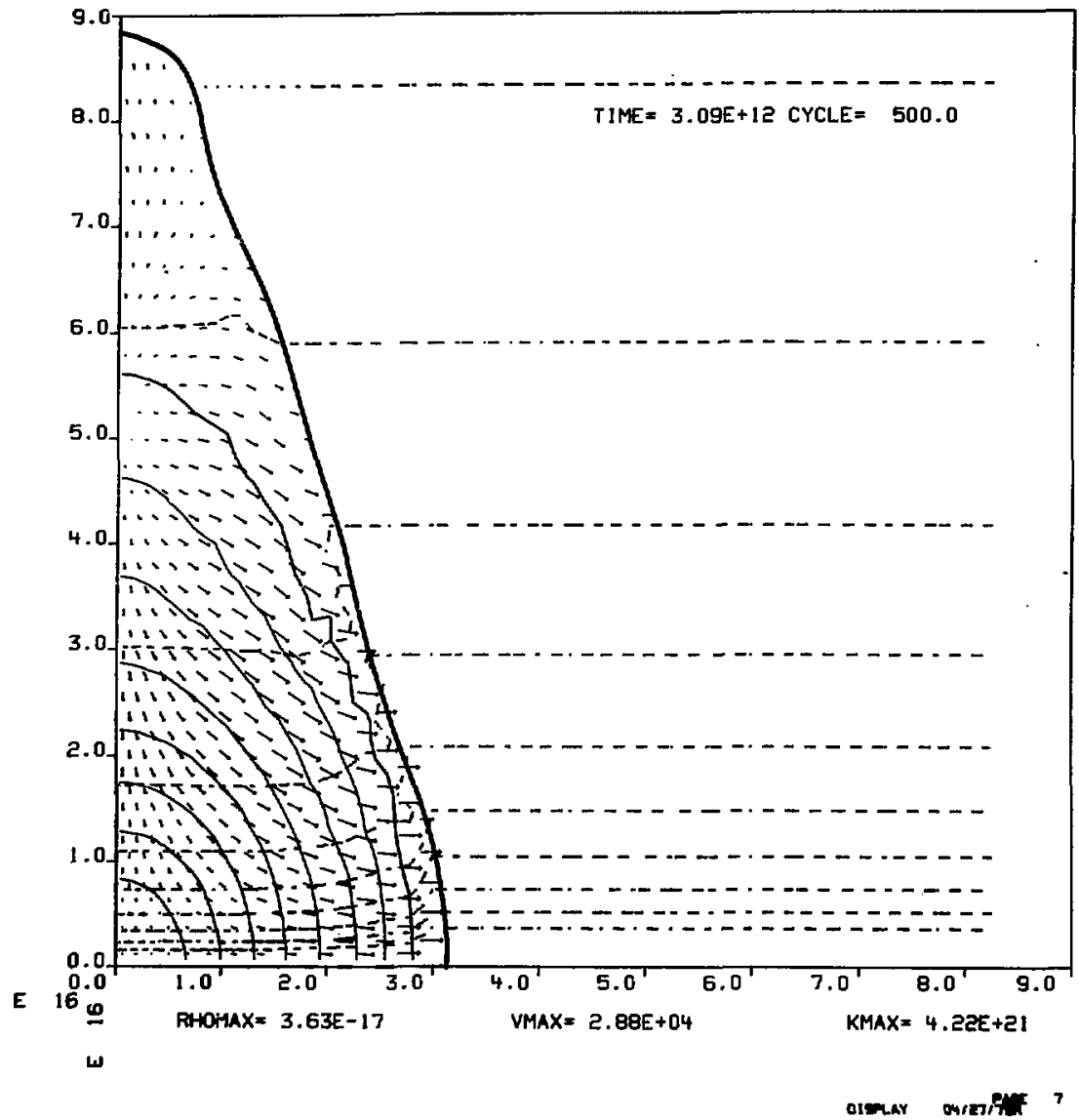

Frame $5 E^{\prime} .3$ 


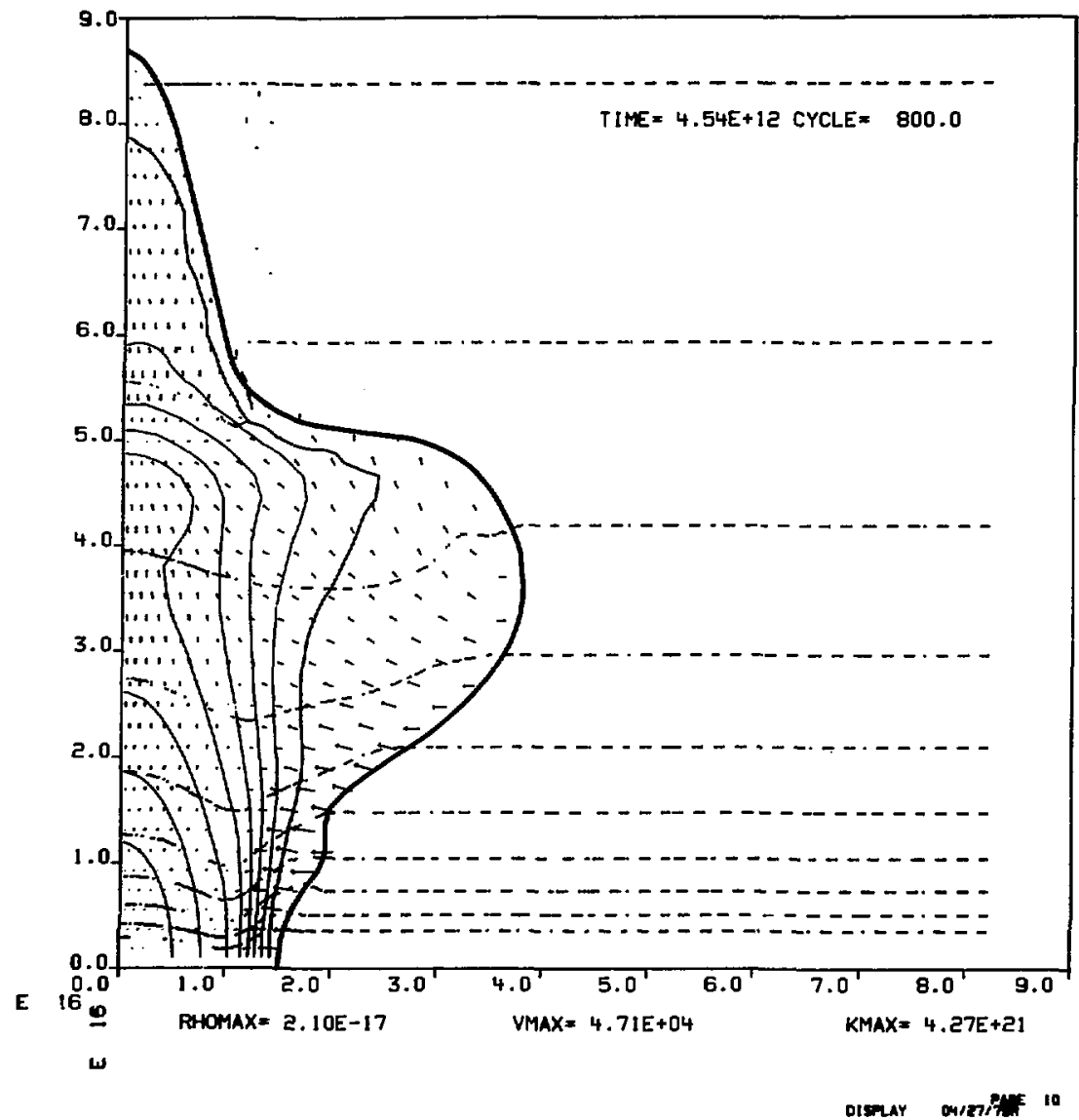

Frame $5 E^{\prime} .4$ 


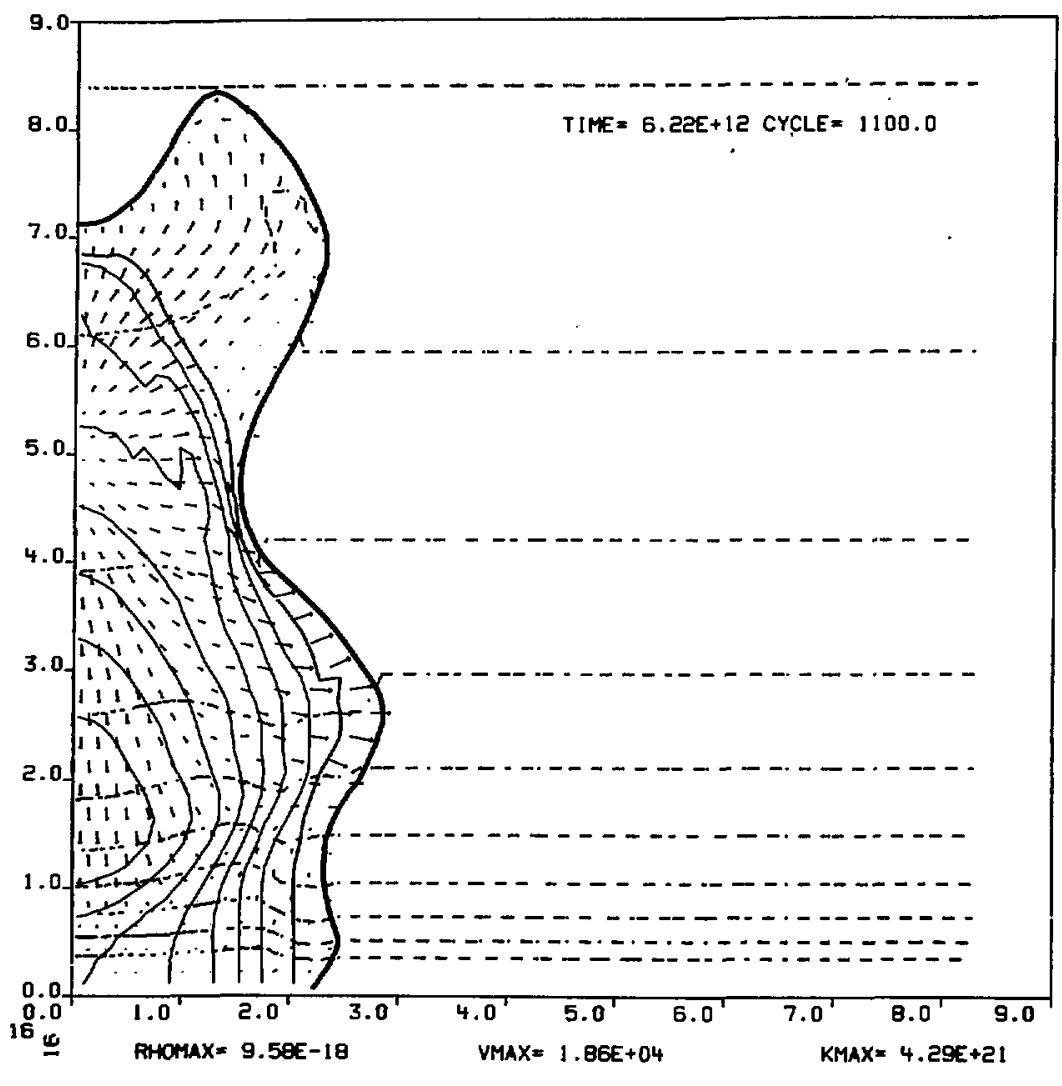

$\mathbf{w}$

Digmar onizhifie 13

Frame $5 E^{\prime} .5$ 


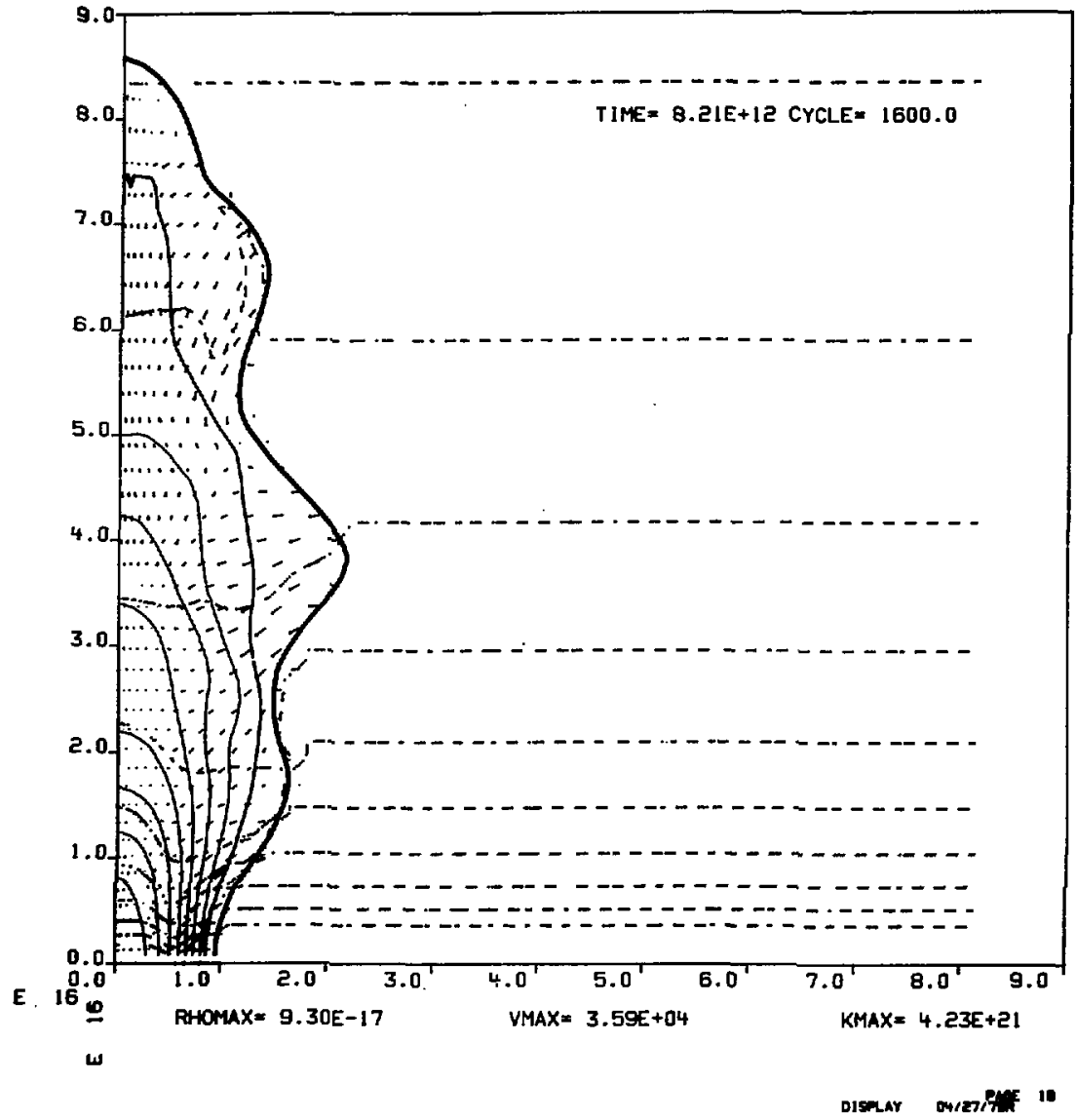

Frame $5 E^{\prime} .6$ 


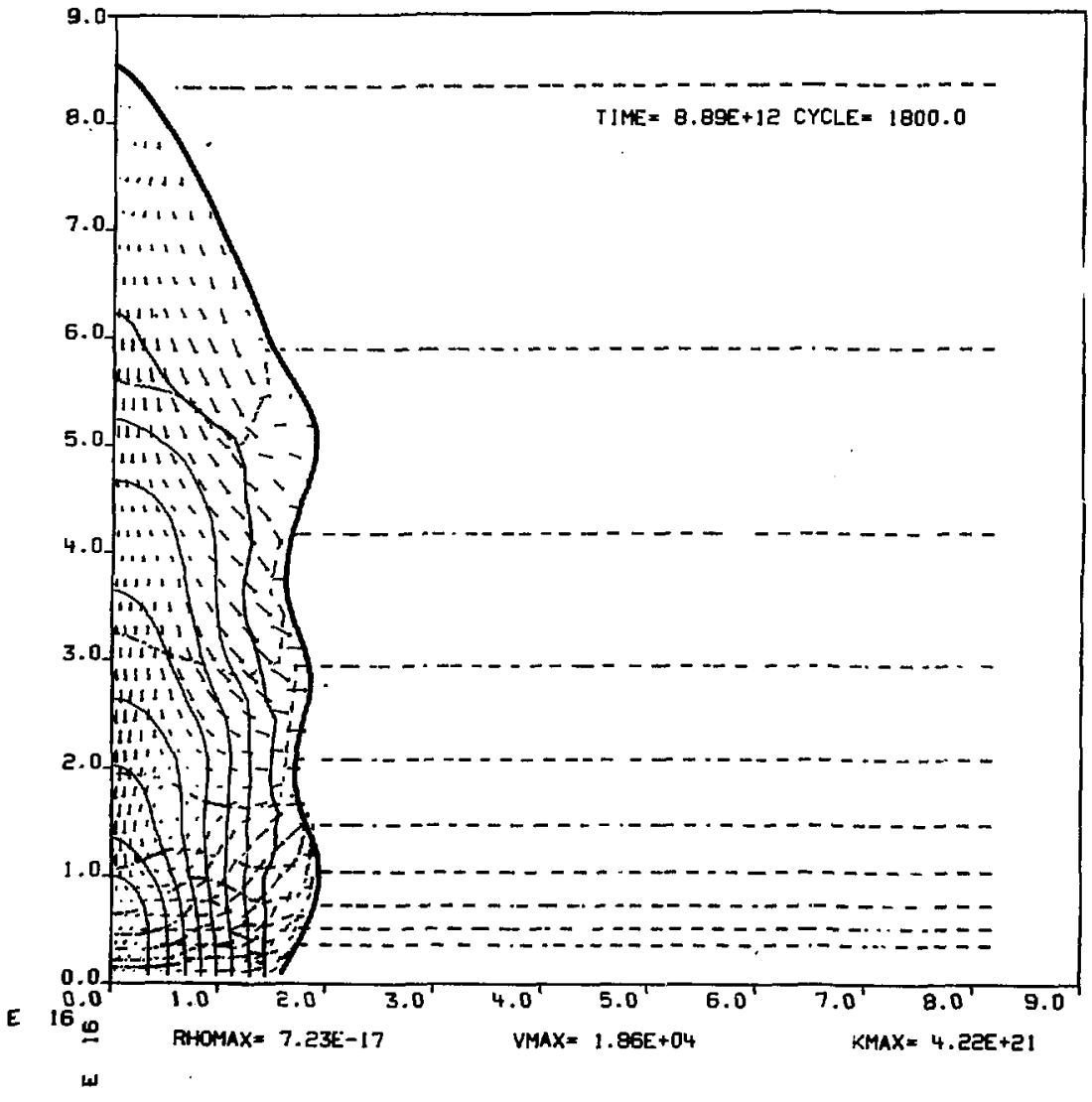

oremar onrezitim as

Frane $5 \mathrm{E}^{\prime} .7$ 


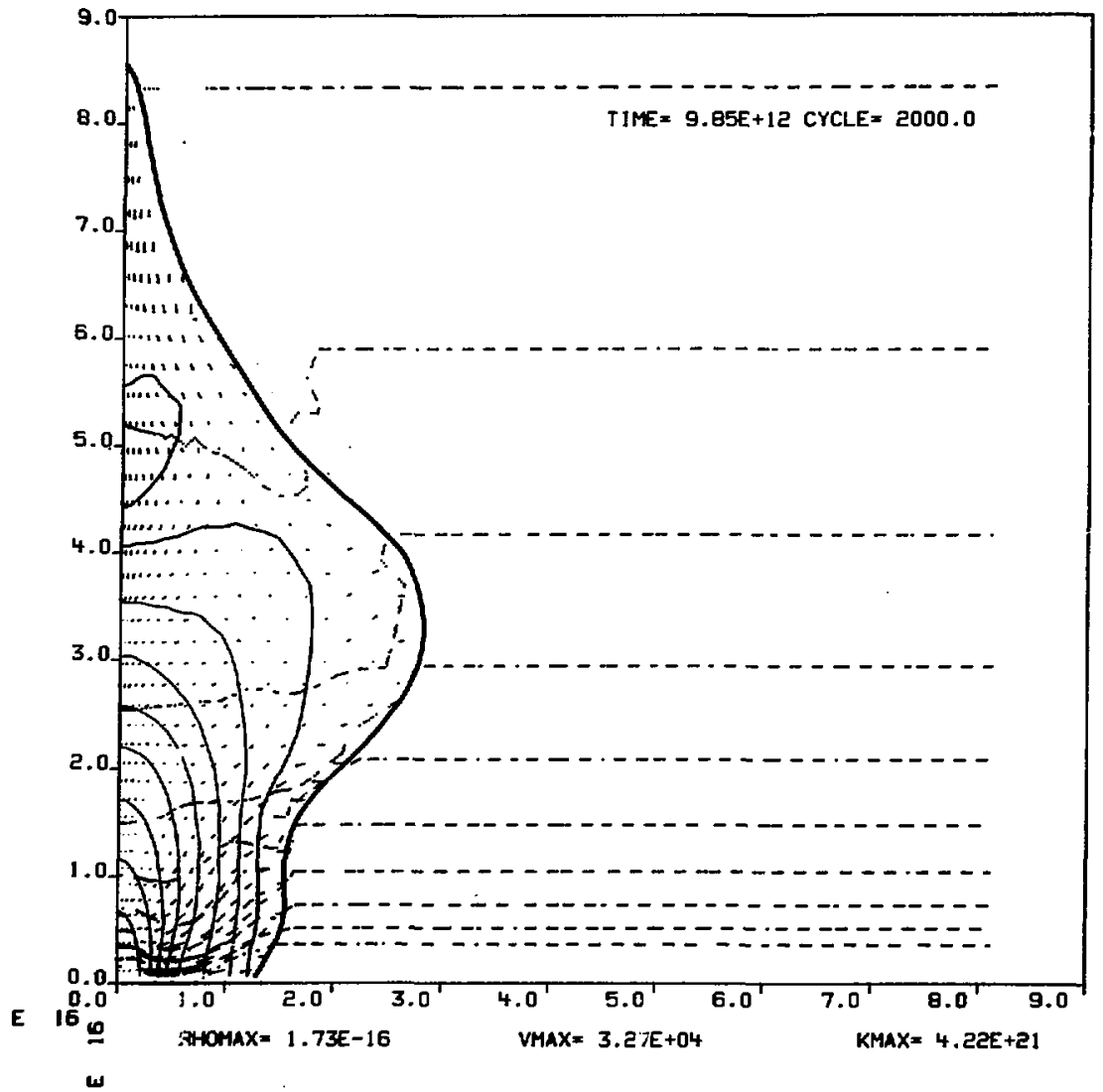

olsmar mistifu a

Frame $5 E^{\prime} .8$ 
Evolution $5 F$

1 


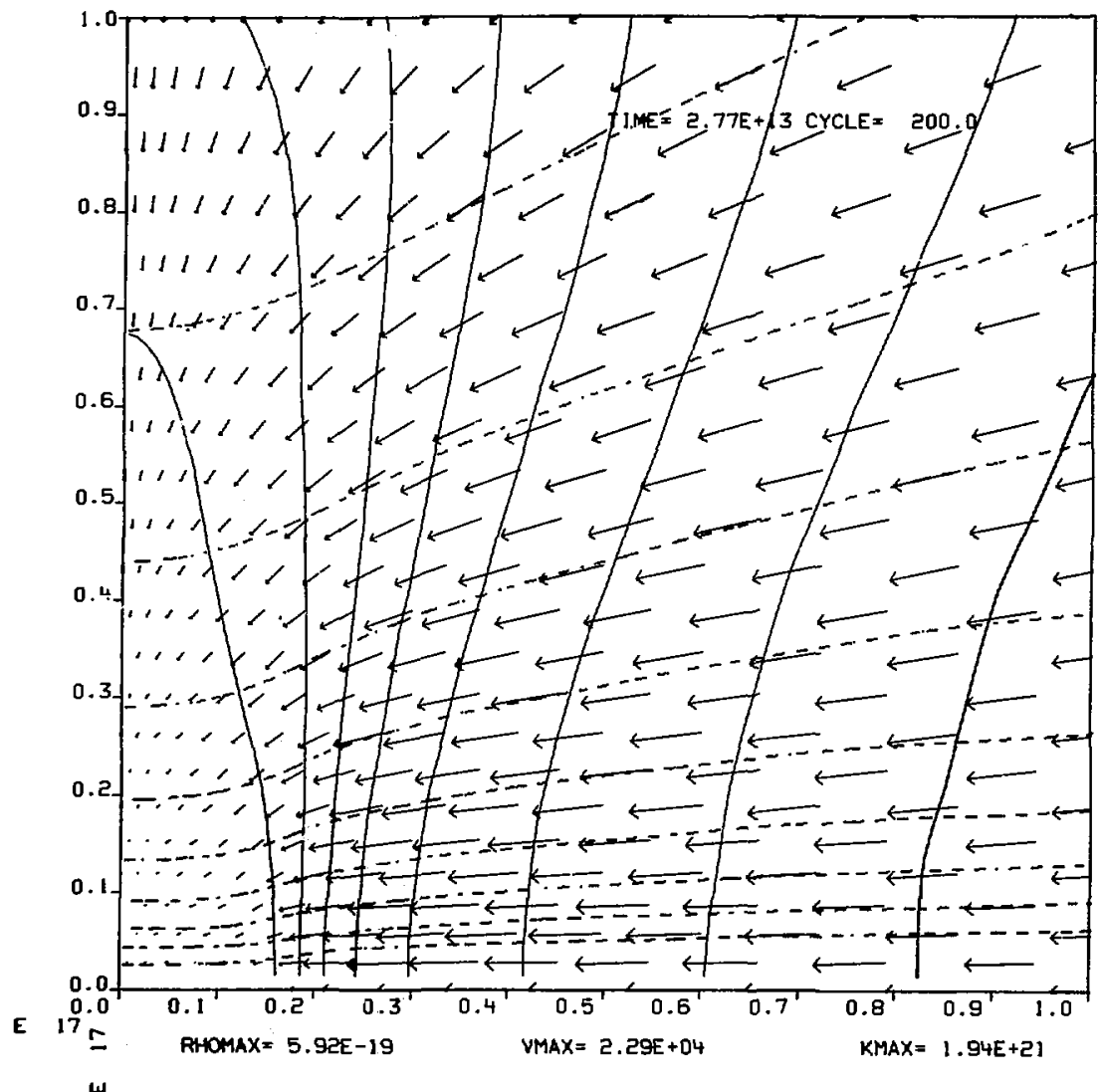

$\boldsymbol{w}$

oIsplar ourepitise 35

Frame 5F. 1 


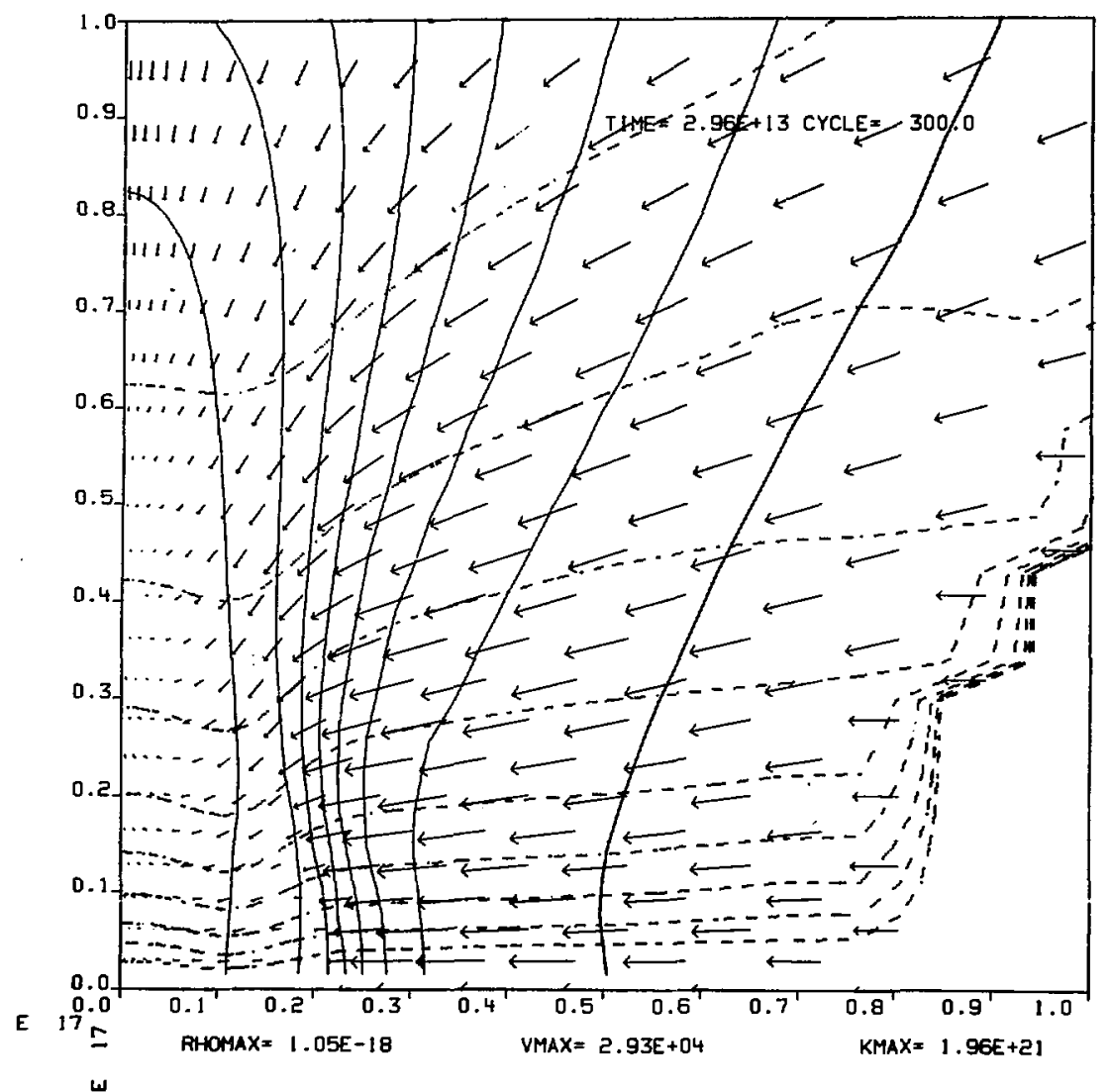

DIselay ondesitix 37

Frame $5 F .2$ 


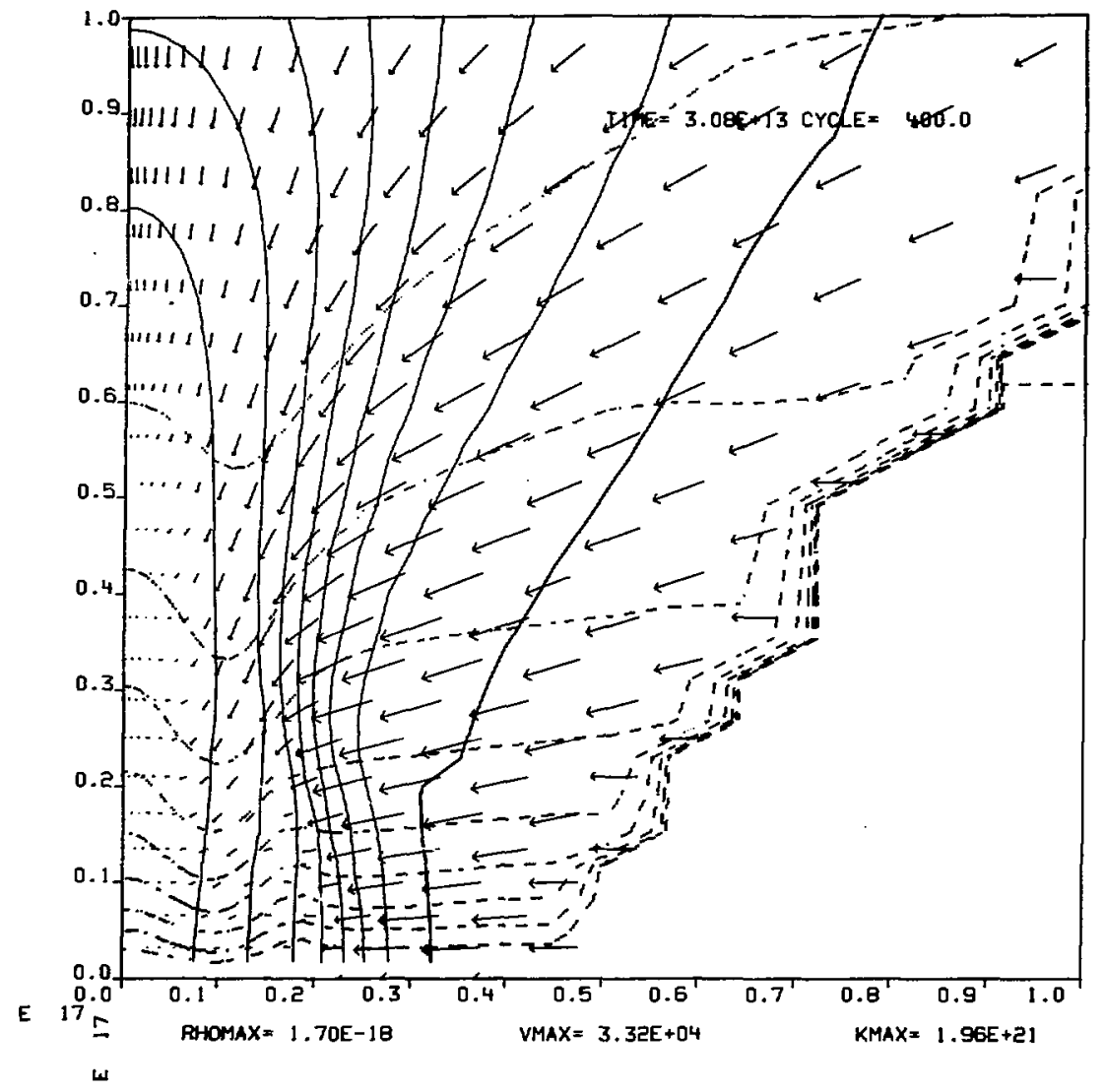

Diselay onrezifiog so

Frame 5F.3 


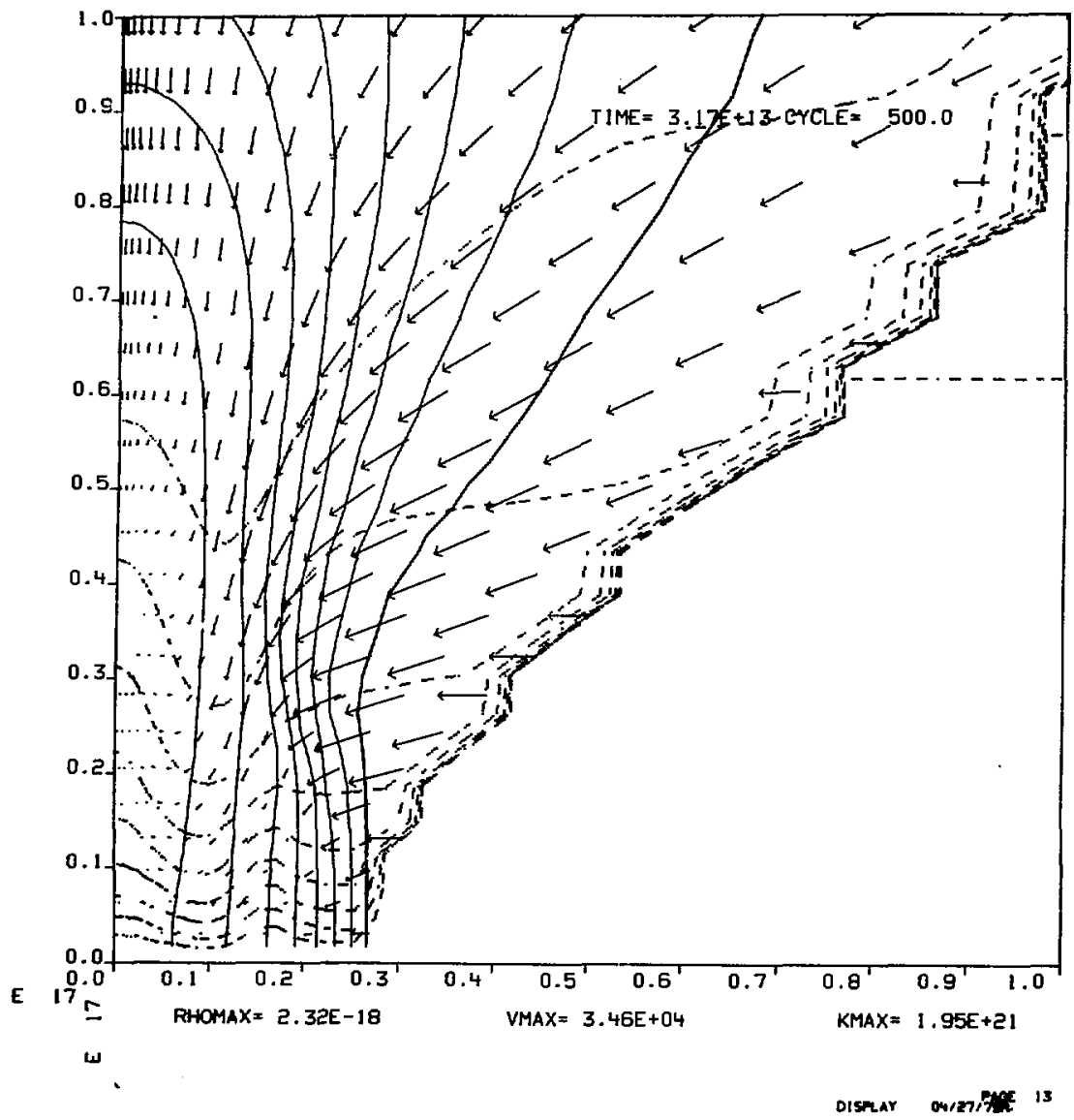

Frame 5F.4 


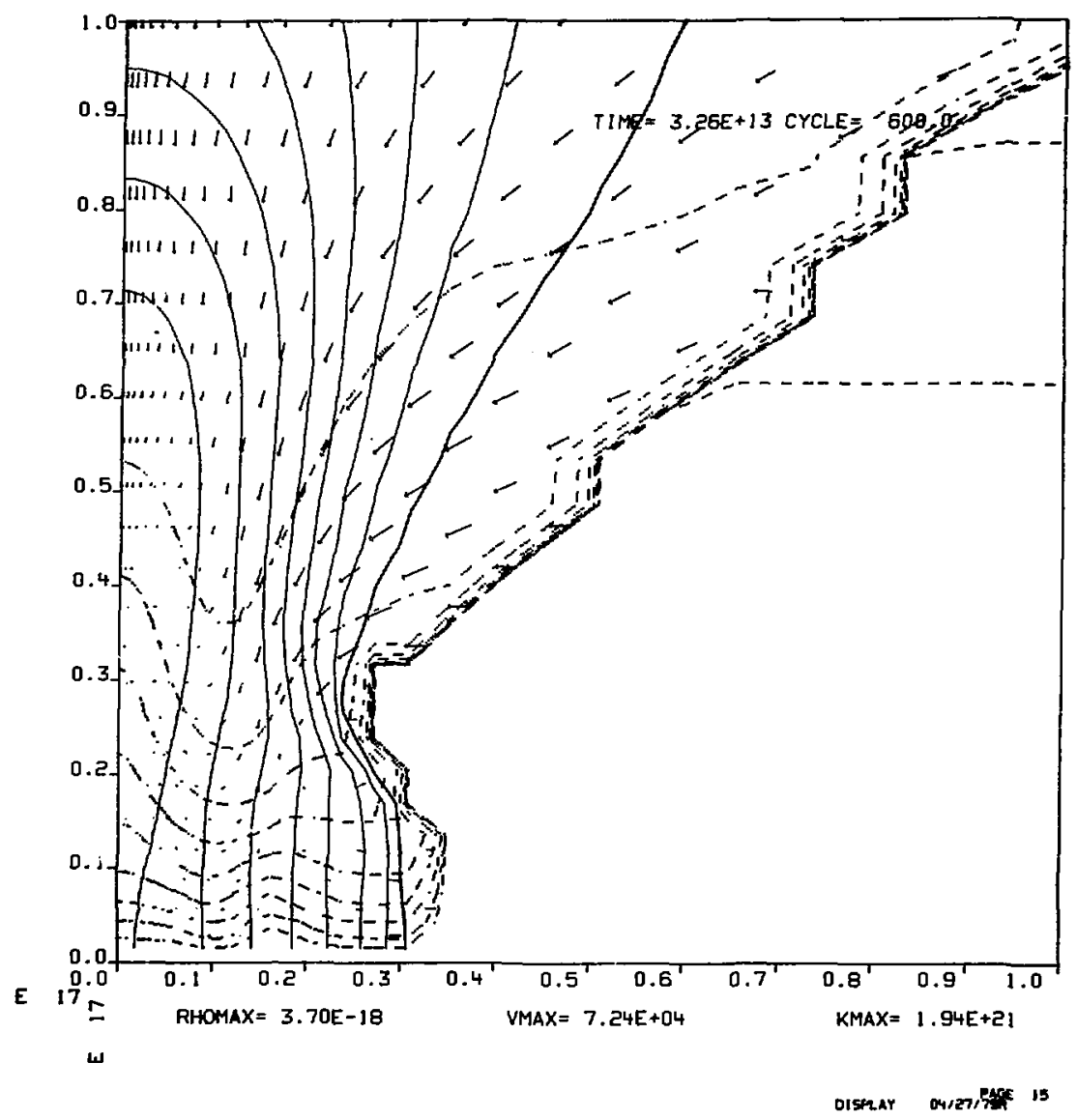

Frame 5F.5 
175

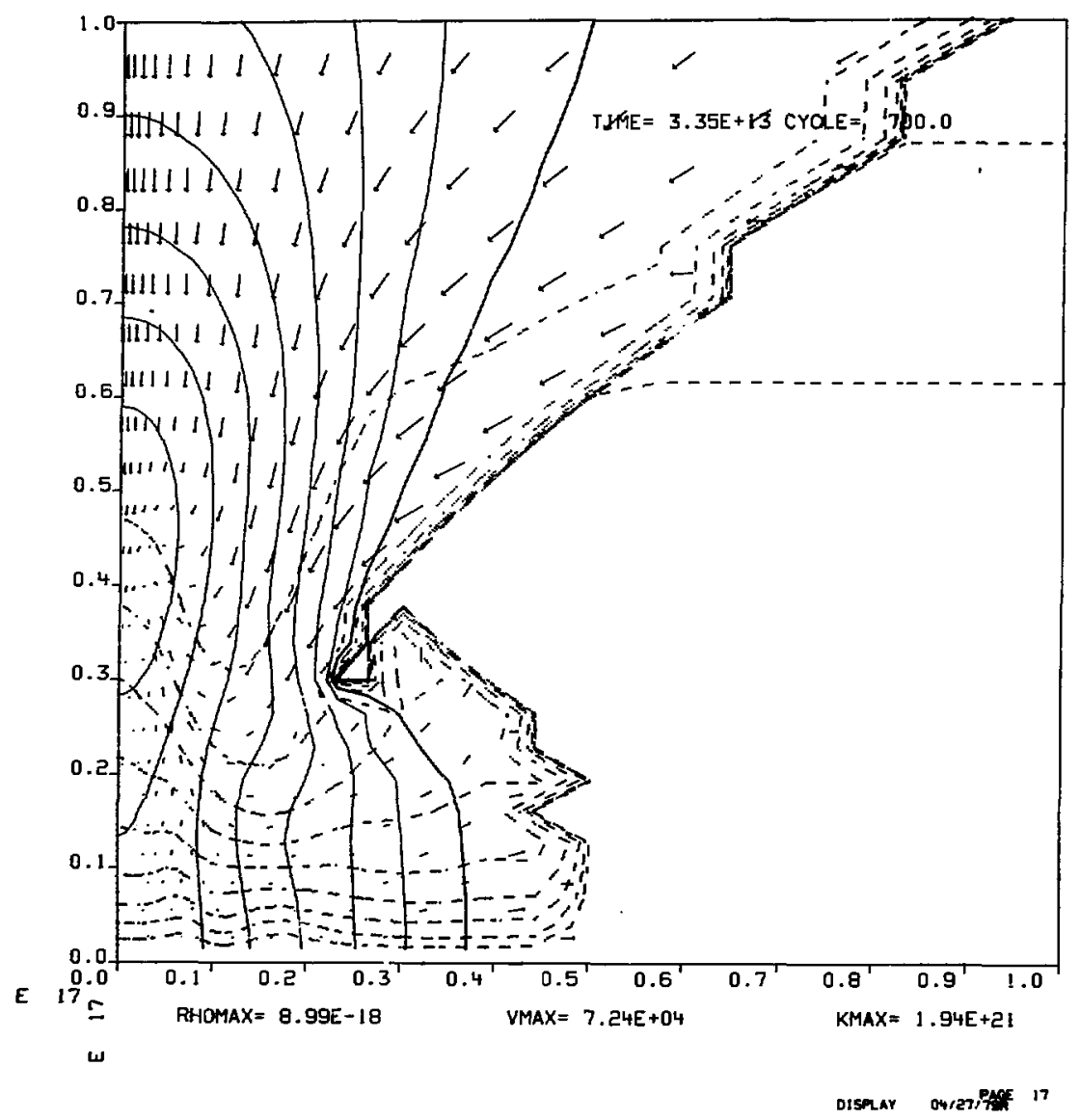

Frame 5F. 6 


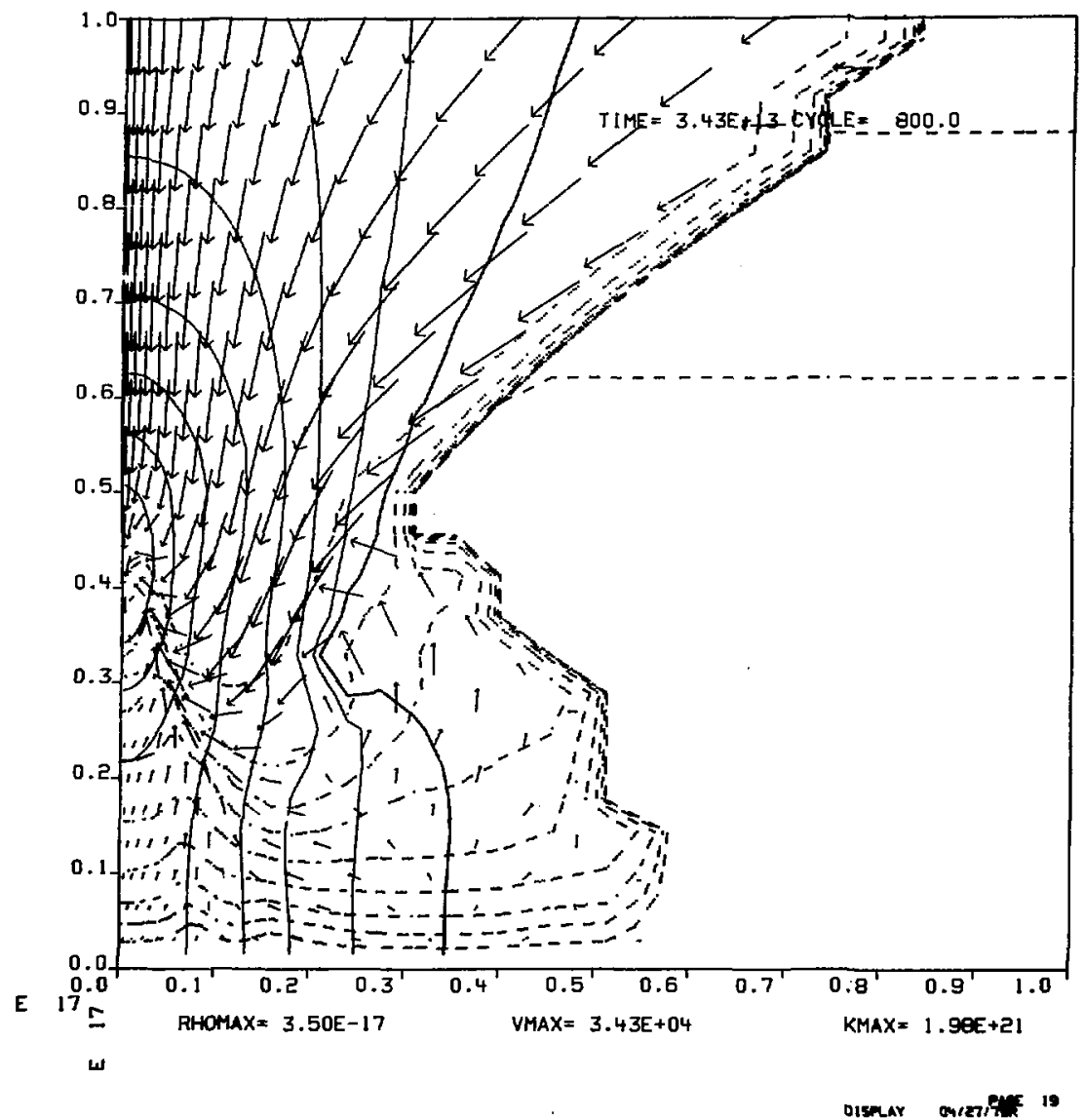

Frame 5F.7 


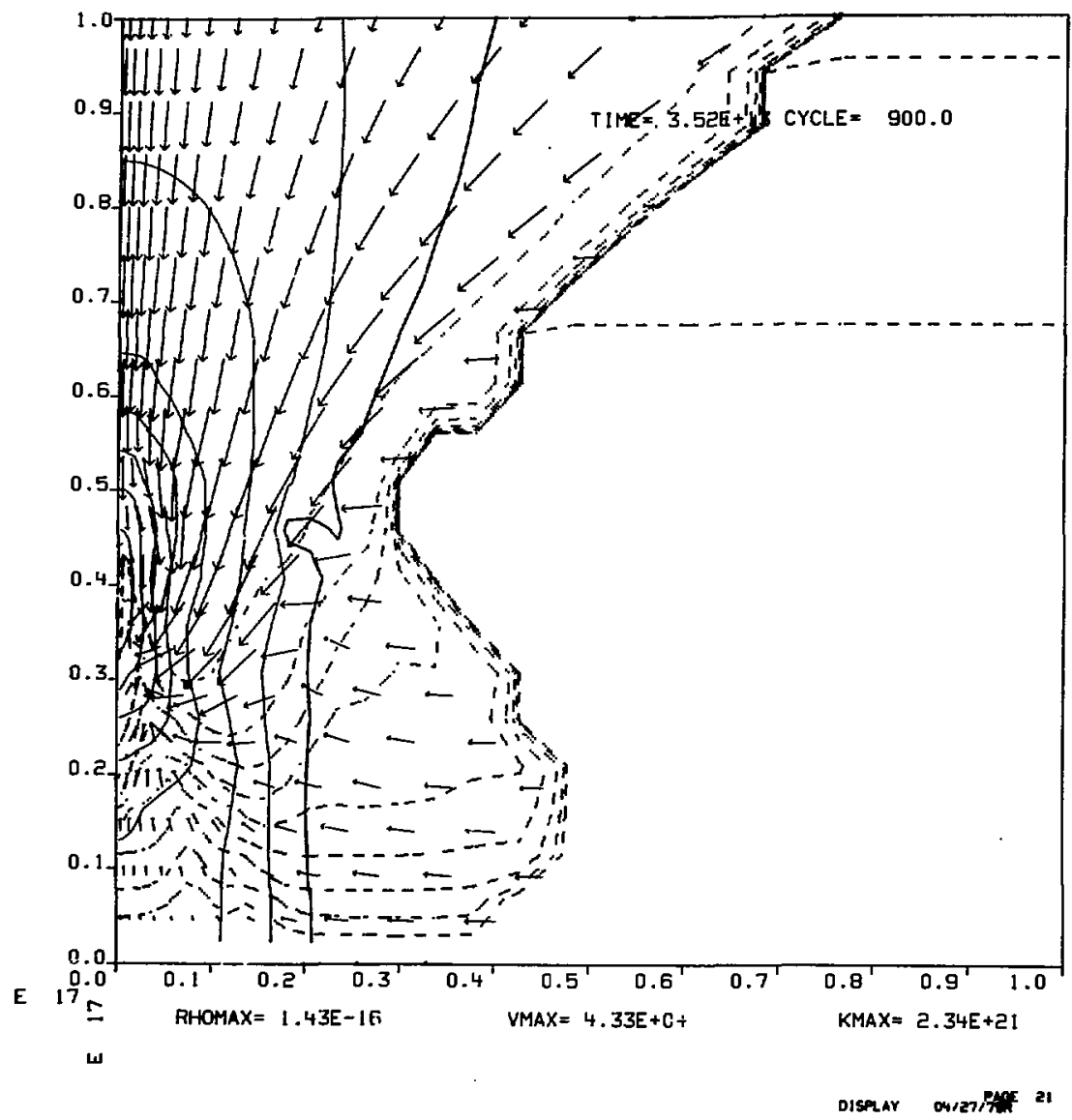

Frame $5 F .8$ 
Evolution 5G 


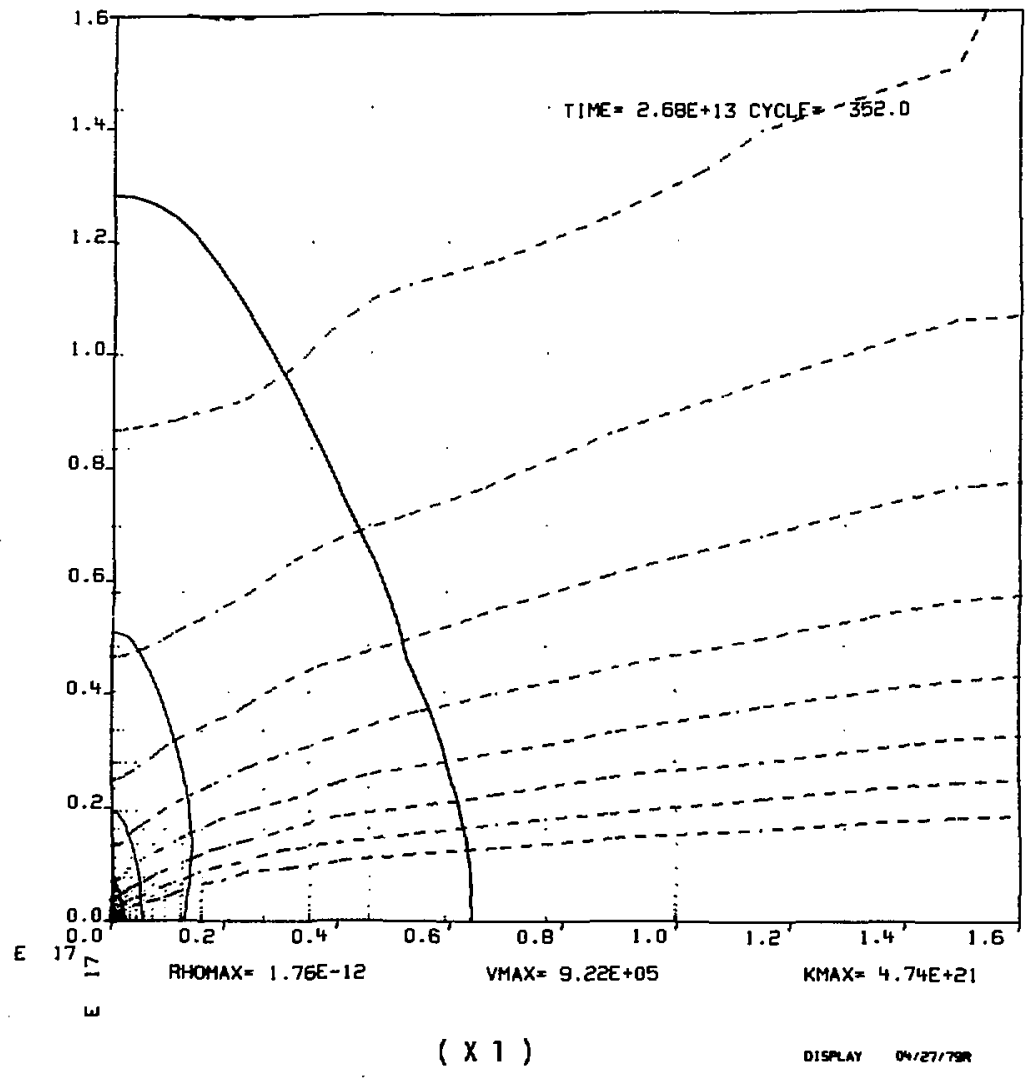

Frame 5G.1 


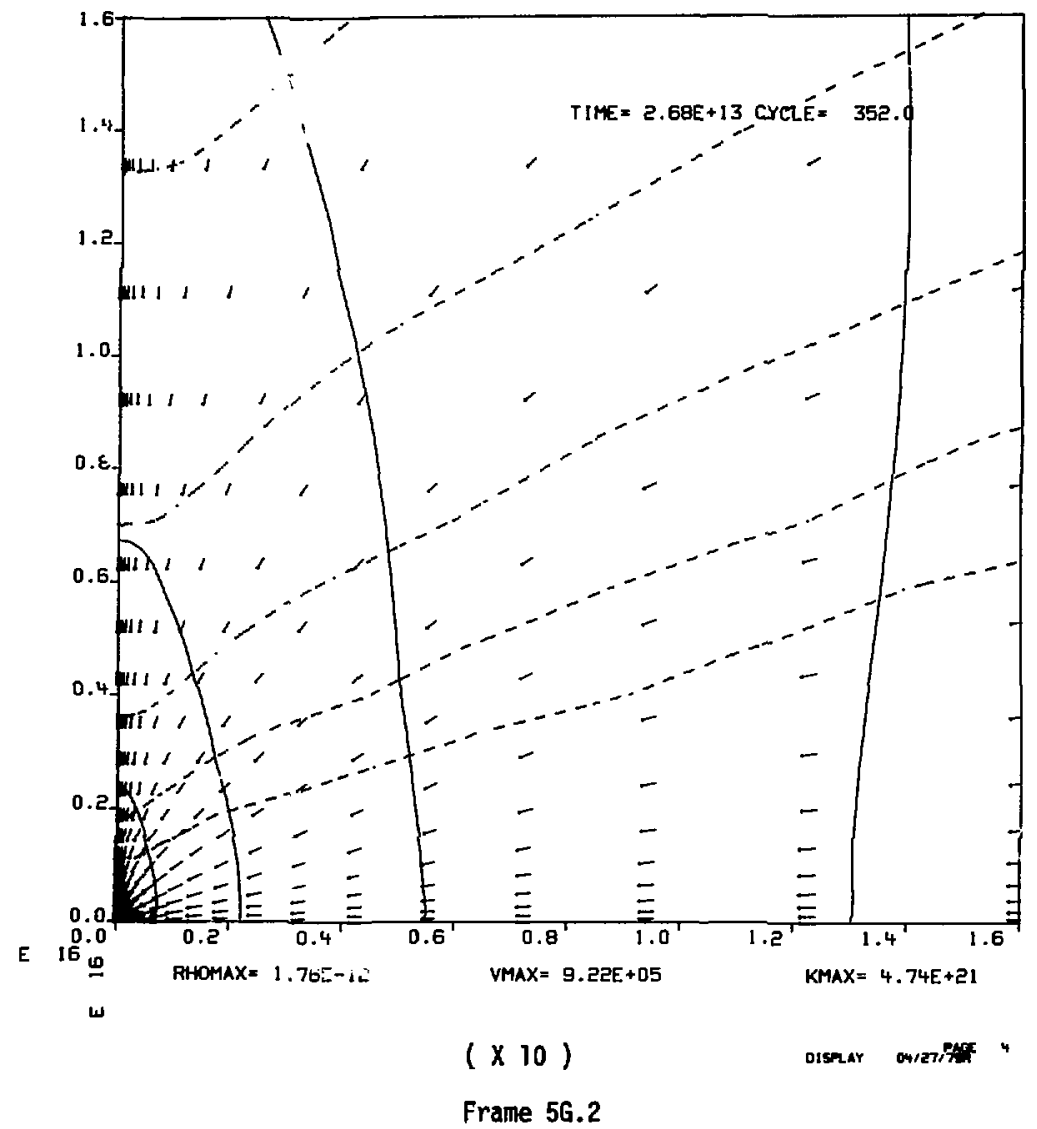




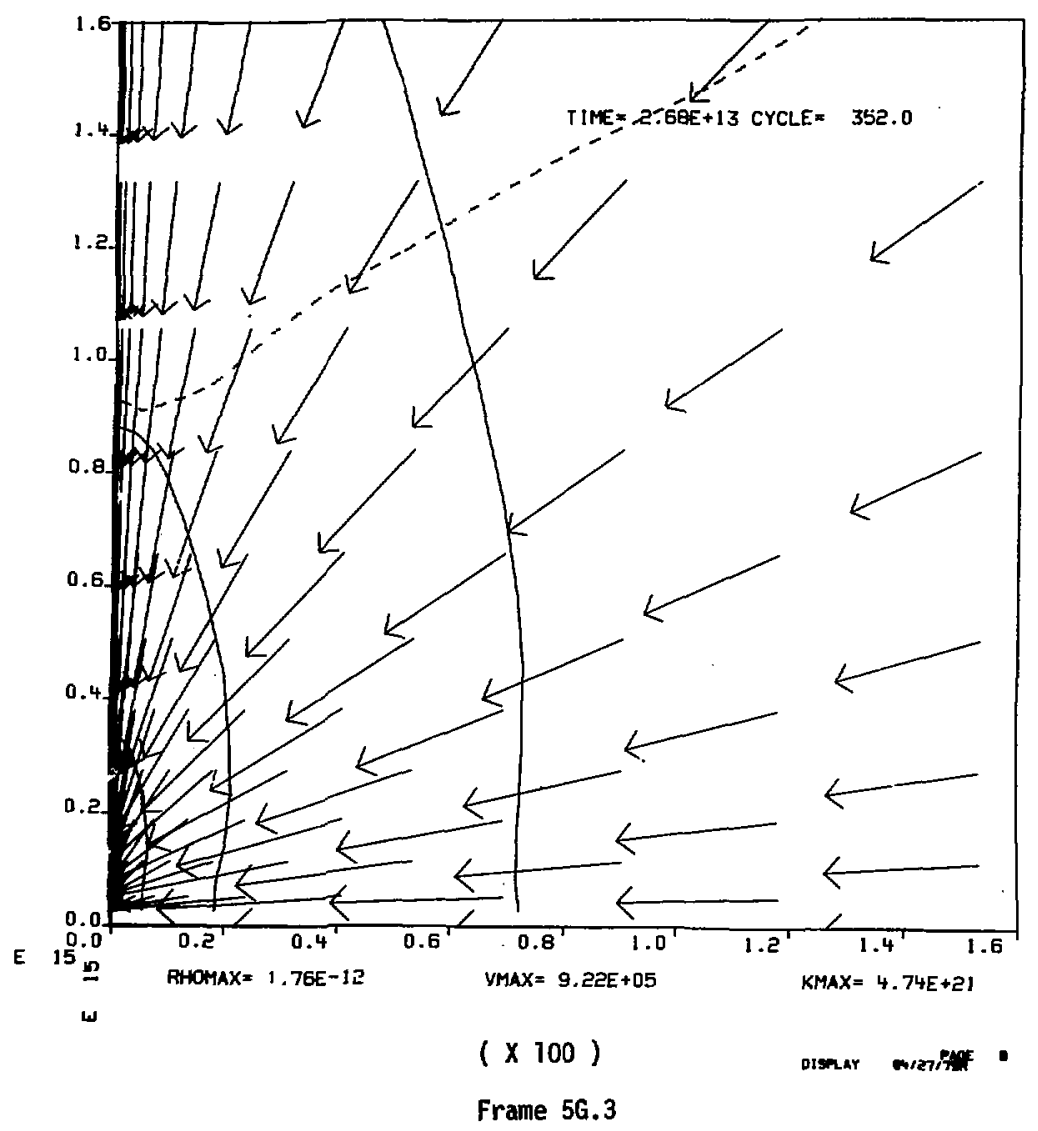


CHAPTER 6

NONISOTHERMAL EVOLUTION

\section{1) Introduction}

Real interstellar mate al compresses nonisothermally when the gas density reaches approximately $10^{10} \mathrm{~cm}^{-3}$ (Gaustad 1983). This is due to trapping of thermal radiation by dust grains so that cooling becomes inefficient. This effect is simulated by putting material on a $\gamma=7 / 5$ adiabat above a trapping density, $\rho_{0}(\sec .2 .1)$.

As collapse proceeds pressure becomes large enough to support a rotating core. The establishment and short-term evolution of this core is calculated for four clouds differing only in their total angular momentum. The dominant feature is the evolution of the core to a ring configuration on a hydrodynamic timescale for higher angular momentum clouds, and on an accetion timescale for lower angular momentum clouds. The critical $\beta$ for ring instability is computed to be in the range $0.37-0.40$.

\section{2) Parameters}

Table 6.1 lists the parameters of the four evolutions. The density and angular velacity are initially uniform $(s=1)$. A constant volume boundary condition is applied. Evolutions 6B, 6C, and 6D start with the same conditions 
as evolutions 5A, 5B, and 5C. The trapping density is $\rho_{0}=10^{-13} \mathrm{~g} \mathrm{~cm}^{-3}$ (see equation 2.1).

TABLE 6.1

PARAMETERS FOR NONISOTHERMAL EVOLUTIONS

$$
\begin{gathered}
\text { Mass: } \mu=\mu_{\theta} \\
\text { Radius: } R_{0}=7 \cdot 10^{1 \mathrm{~B}} \mathrm{~cm} \\
\text { Sound Speed: } a=2 \cdot 10^{4} \\
\text { Density: } \rho_{0}=1.30 \cdot 10^{-18} \mathrm{~g} \mathrm{~cm}-3 \\
\text { Boundary Condition: Constant Volume }
\end{gathered}
$$

Designation

6A

$6 B$

BC

BD

$$
J\left(10^{54} \mathrm{~g} \mathrm{~cm} \mathrm{~s} \mathrm{~s}^{-2}\right)
$$

$\mathbf{s}$

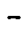

1
1

1

1.69

0.30

0.60

1.20

1
Zones

$48^{2}$

$48^{2}$

$48^{2}$

$48^{2}$ 


\section{3) Results}

Figure 6.1 shows the time history of the central and maximum densities during core formation for the four cases. Obvious features are that the more slowly rotating clouds evolve to higher density at earlier times. Evolution to the $r$ ing state begins when the central density (solid 1 ine) and the peak density (dashed line) rapidly diverge. Core $\beta$ and mass $f$ raction, $m$, are indicated at select times by an arrow. ${ }^{l}$ One sees that $m$ and $\beta$ is larger at $f$ irst bounce for the higher angular momentum clouds. $\beta$ at the moment of $r$ ing formation ranges between 0.37 and 0.40 . Case $6 \mathrm{~A}$ remains a disk for the duration of the calculation, while cases $6 B, 6 C$, and $6 D$ each evolve to a $r$ ing on increasingly rapid timescales (in terms of core oscillation period). Details of the core motion are now described for two representative cases.

\section{Low Angular Momenlum Core: $6 \mathrm{~A}$}

Frames 6A.1 through 6A.5 show isodensity plots of the core at vartous times in the evolution. The slight rounding at the center of the disk in frame $6 \mathrm{~A} .1$ shows that pressure is reducing flattening (c.f., frame 5A.3). A shock at $Z \sim$ $10^{14} \mathrm{~cm}$ decelerates material flowing down the axis at 5 times the sound speed. By Irame $6 \mathrm{~A} .2$ a shock front of varying strength extends to $\mathrm{R} \sim 10^{14} \mathrm{~cm}$ following the density lines. The density and angular velocity are still monotonic functions of distance from the center. A "plug" of accreted material is seen in frame 6A.3, which eventually collapses (frame 6A.4), sending a pressure disturbance out from the rotation axis. At this time the radial velocities have become positive for $R<8 \cdot 10^{13} \mathrm{~cm}$, making $\Omega$ peak at the focus of the two streams. 1 Mass fraction and $\beta$ are integrated numerically interior to the surface of the core, defined by $p=\rho_{0}$. 

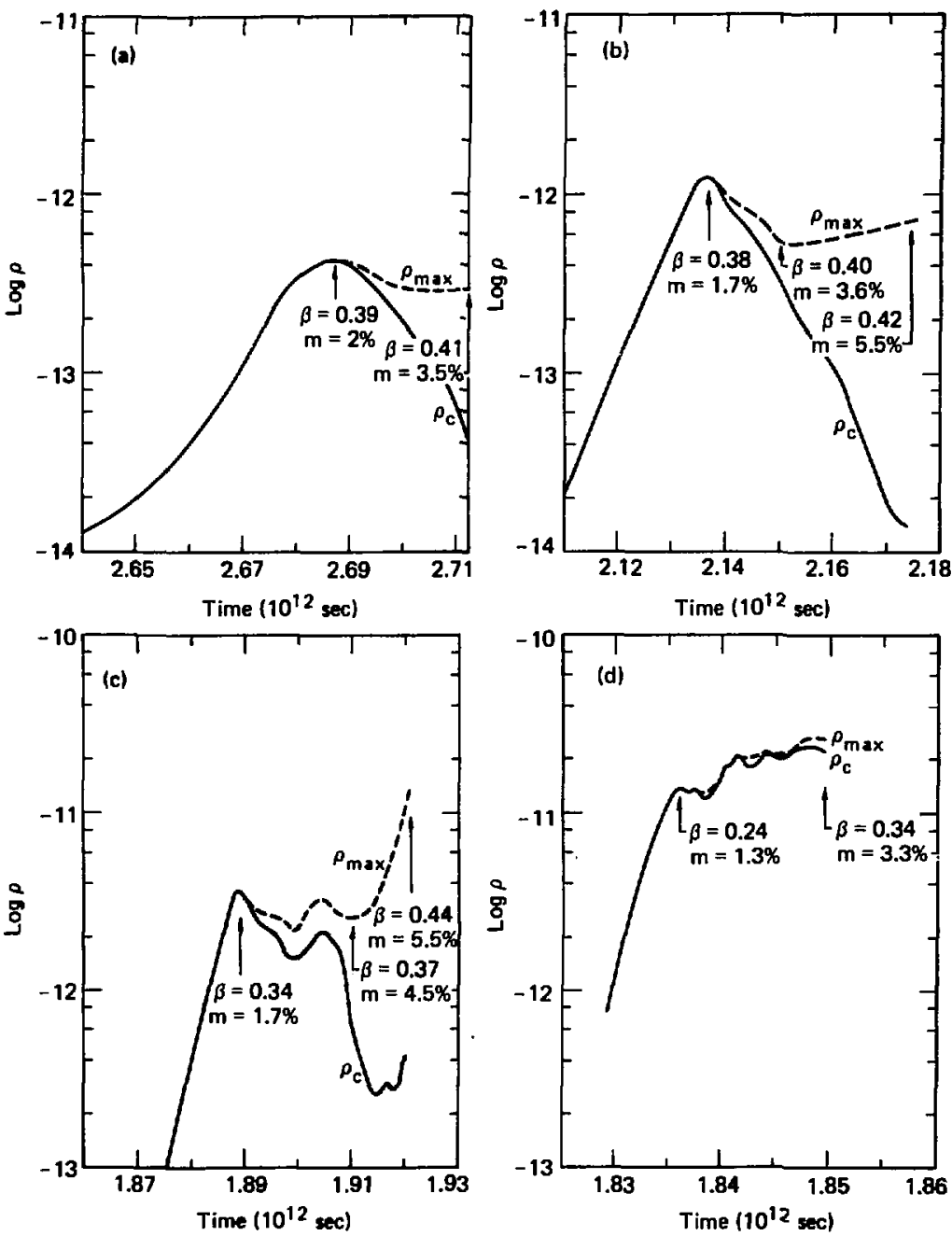

Figure 6.1--History of central density (solid line), and maximum density (dashed line), for core formation in clouda of different angular momenta. (a) $6 \mathrm{D}$, (b) $6 \mathrm{C}$, (c) $6 \mathrm{~B}$, (d) $6 \mathrm{~A}$. 
Subsequen!ly the core executes a damped oscillation characterized by alternately positive and negative radial veiocities synchronized with alternately negative and positive velocity components down the rotation axis. The core continues to grow in mass, rotational energy, and extent. Frame $6 \mathrm{~A} .5$ shoes the core at the end of the calculation. The barrier is economic. The slow growth of an of $f$-axis density maximum and the accompanying increase in $\beta$ points to eventual ring lormation provided accretion continues. The final core temperature is 87 K.

\section{High Angular Momentum Core: 6C}

Here the core turns quickly into a ring. Frames 6C.1 to 6C.4 show the core making a rath: smooth transition from disk to ring without the obvious oscillations exhibited by the lower angular momentum models. Core $\beta \sim 0.38$ at bounce time, which is above the value at which case 6 B evolved to a $r$ ing. We therefore expect ring formation on a hydrodynamic timescale. Peak temperature at the end of the calculation is $22 \mathrm{~K}$.

\section{4) Comments}

Boss (1980) has recently computed the collapse of rotating polytropic clouds, and finds $\beta \sim 0.43$ for $r$ ing formation. The present calculations are the same sort recast in the protostellar setting. Our critical $\beta$ of $\sim 0.40$ is in fair agreement with his number considering differences in zoning, method, and editing. Tscharnuter (1978) reported ring growth in the opaque core of a 3 solar-mase protostar for $\beta \sim 0.36$. His calculation included radiation flow and some mechaniem for angular momentum redistribution. This may account for the 
difference, although a 20 percent spread between the three values must be considered good agreement at this early stage of investigation.

Tohline (1980) has stated that pressure may inhibit ring formation in protostellar collapse, as indeed it would if $\beta_{\text {core }} \ll 0.4$. However we see that pressure may also help ring formation if $\beta_{\text {core }}$ is high. 
Evolution BA 


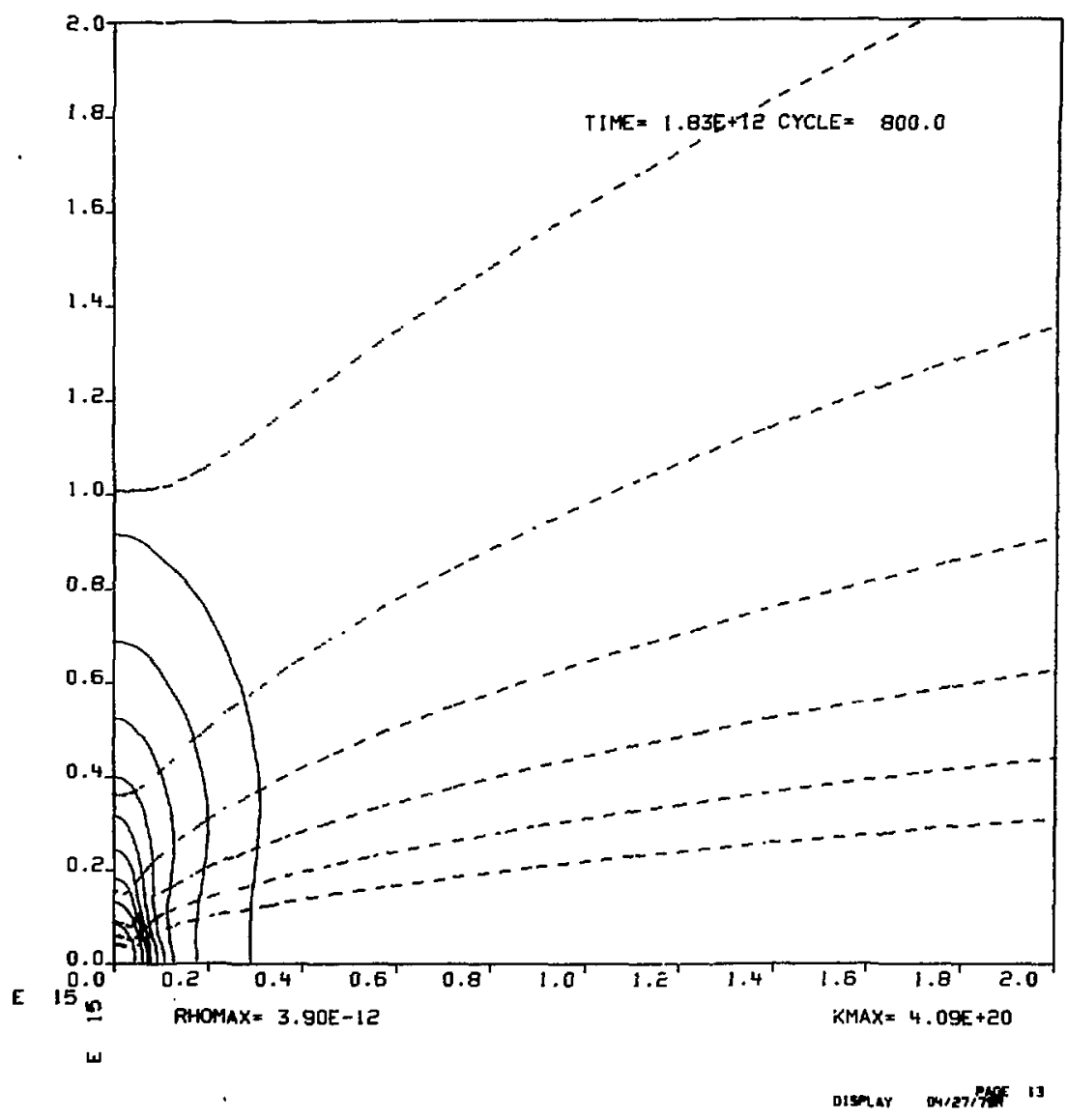

Frame 6A.I 


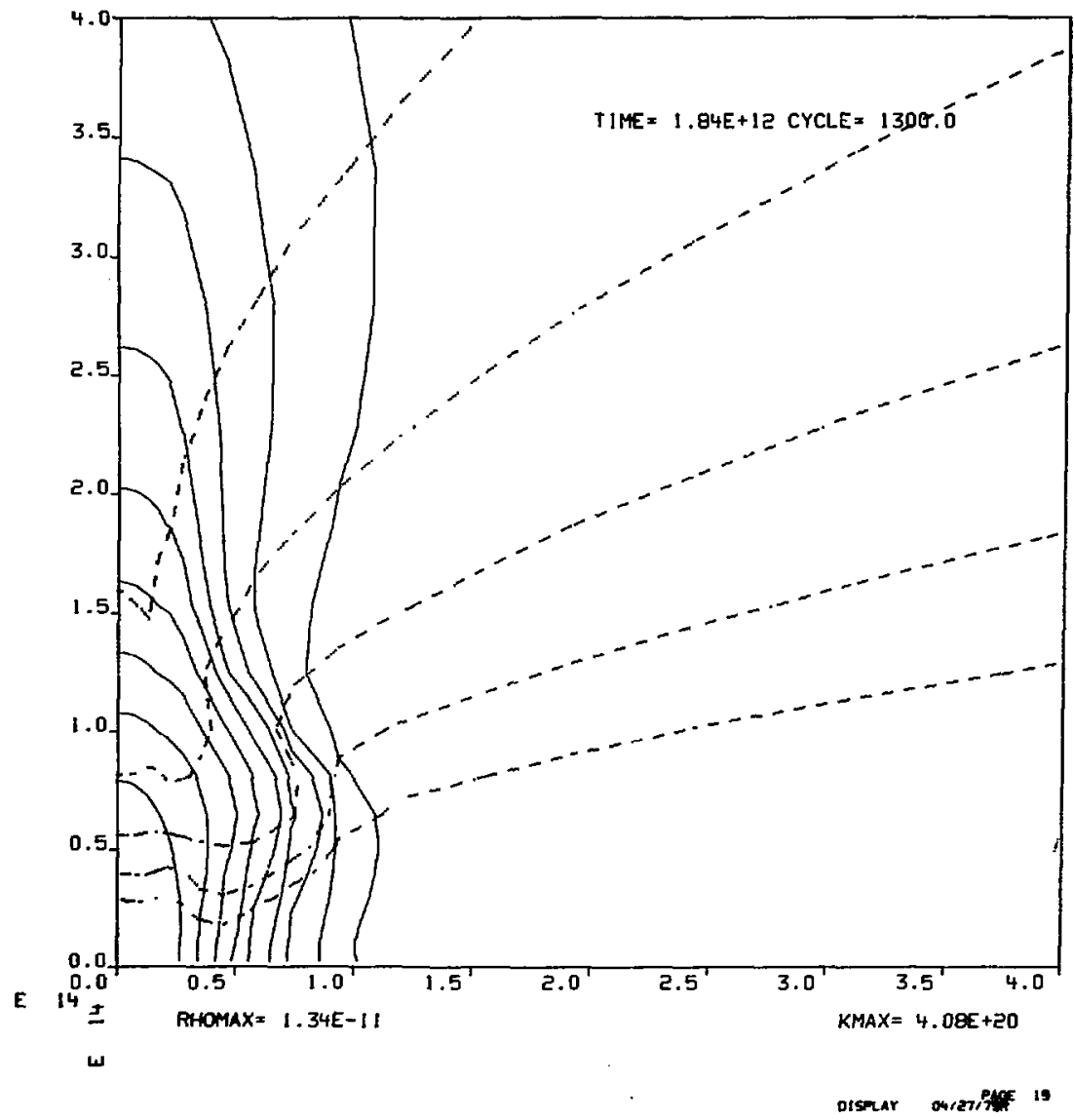

Frame 6 A.2 


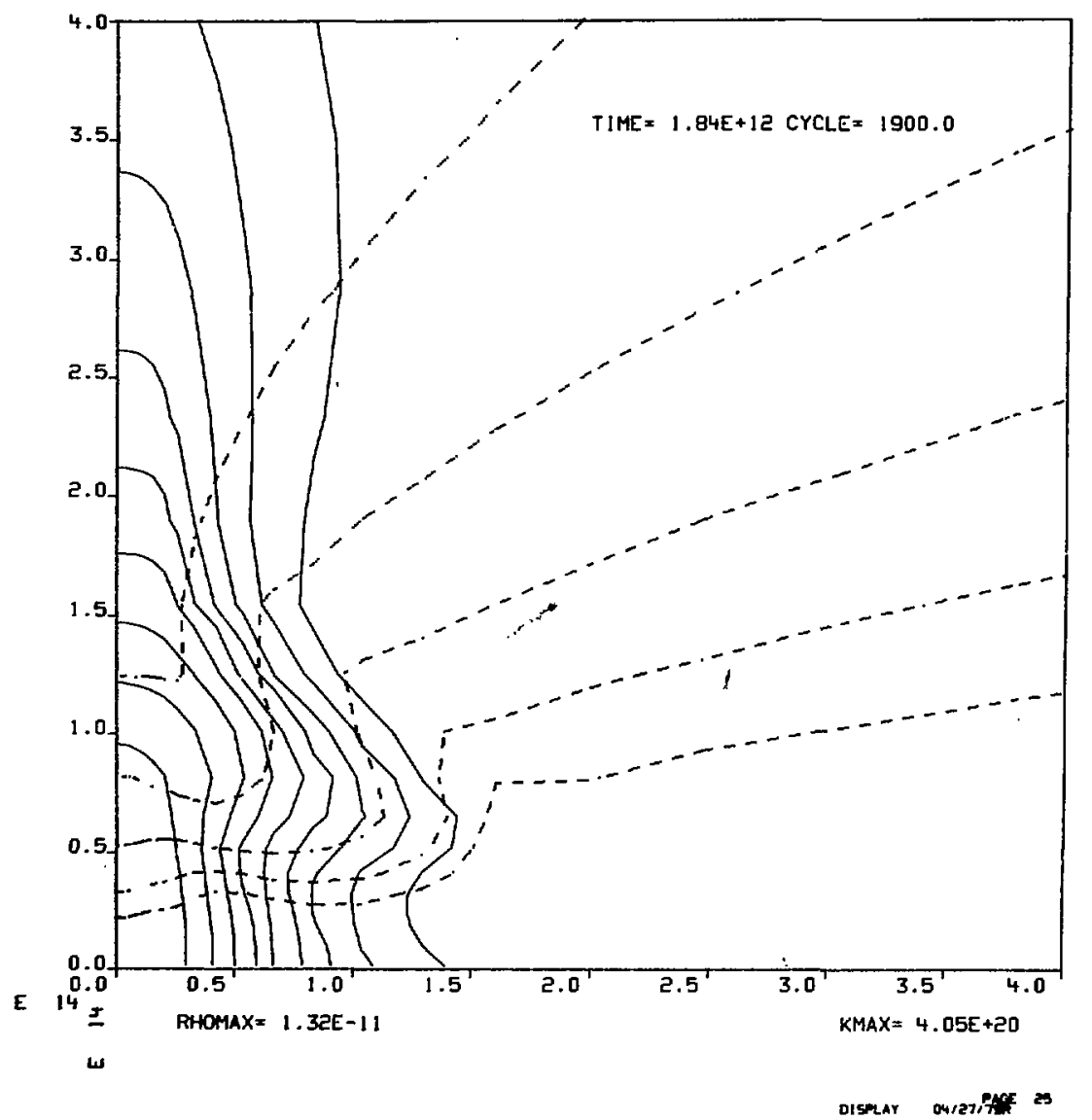

Frame 6A.3 


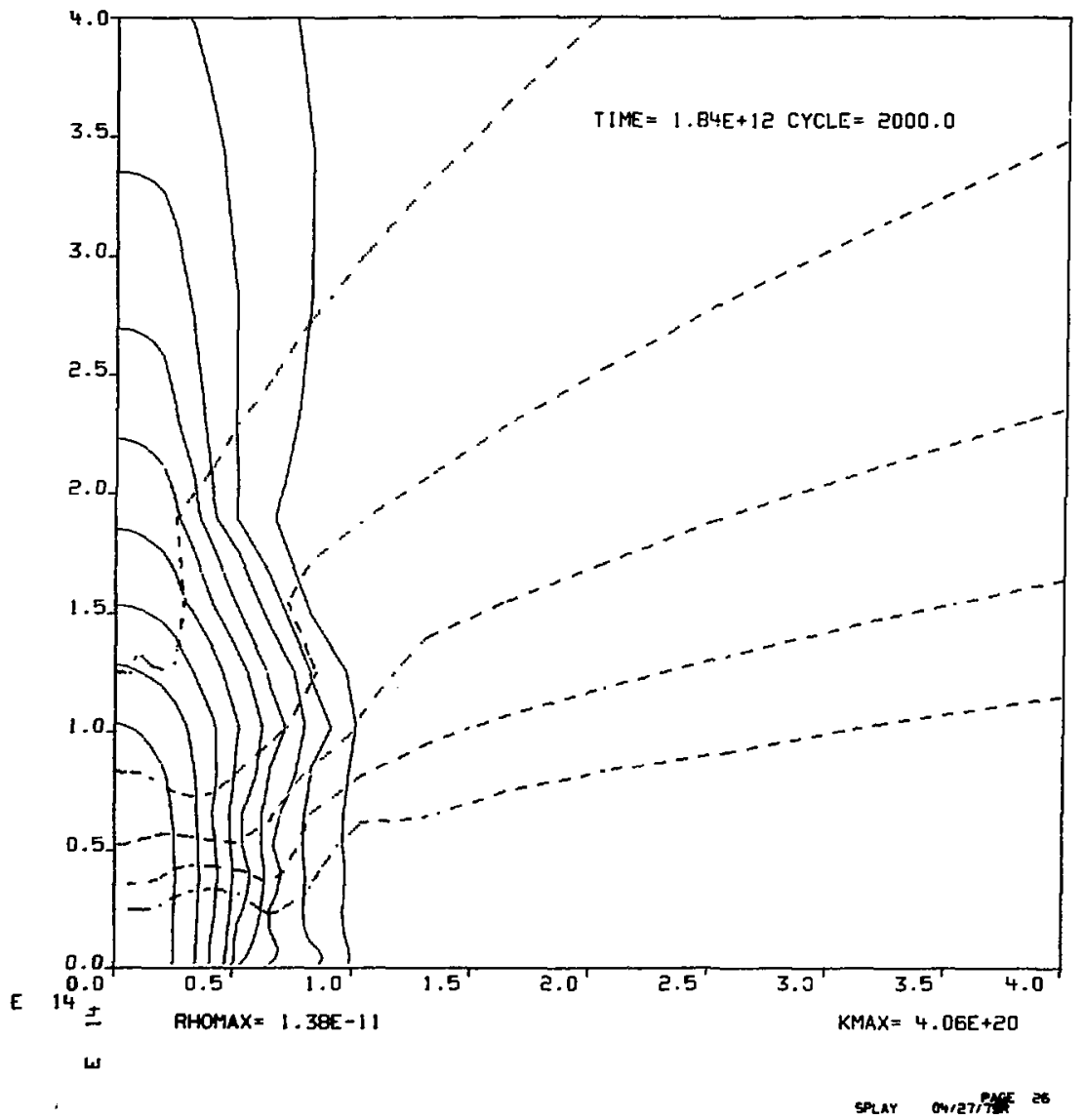

Frame $6 \mathrm{~A} .4$ 


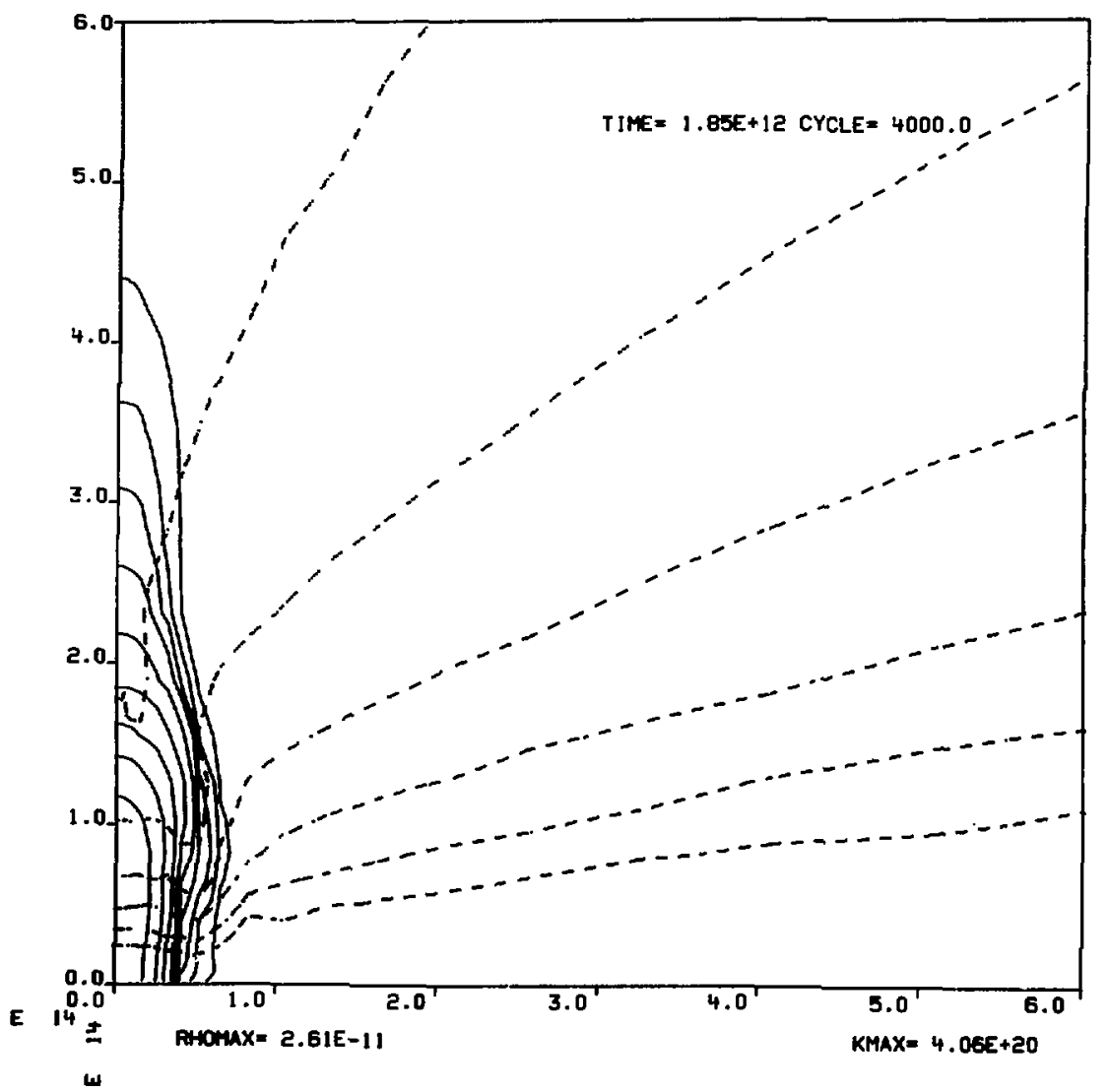

ormeray ererifis 43

Frame $6 A .5$ 
Evolution 6C 


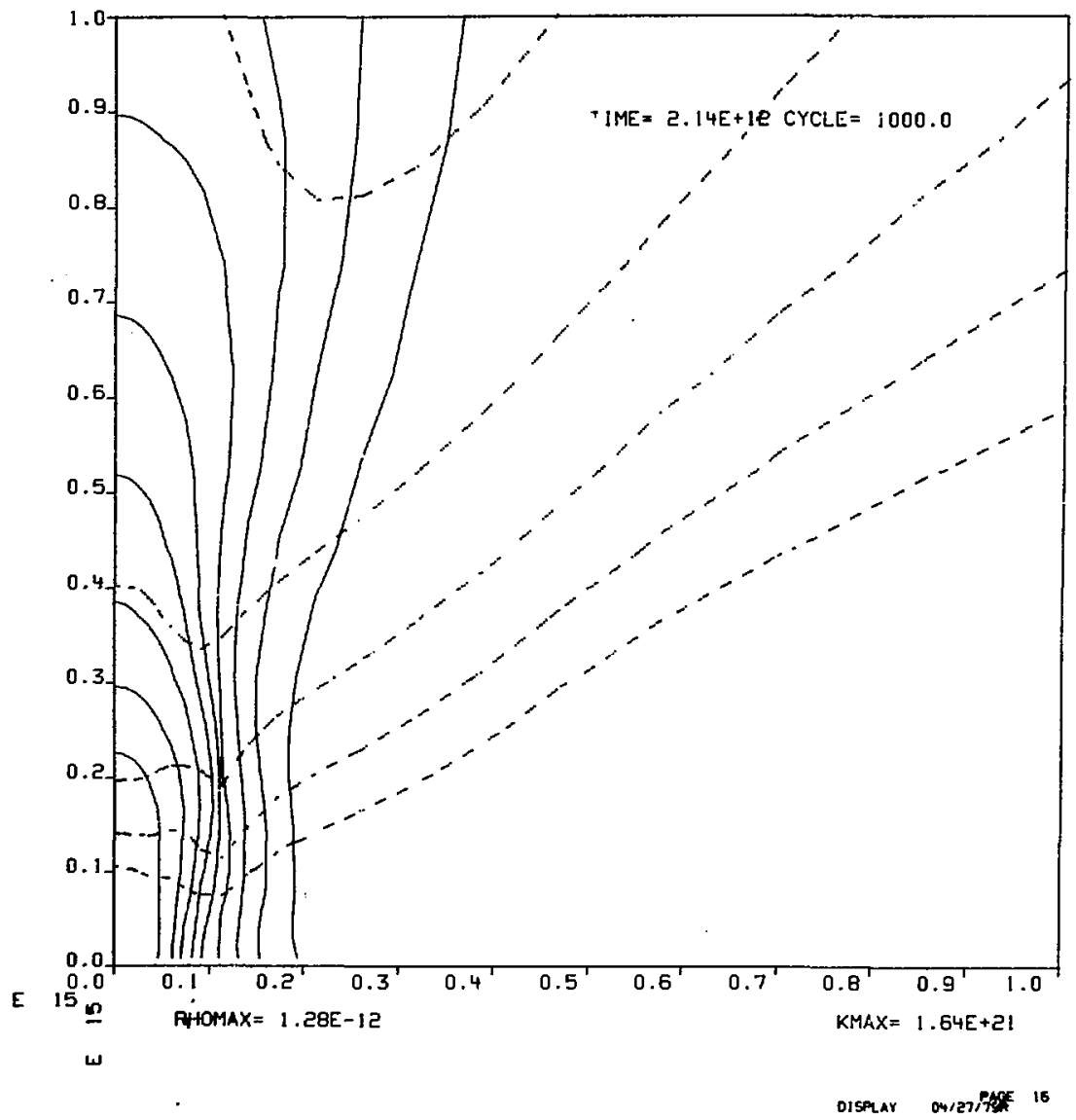

Frame 6C.I 


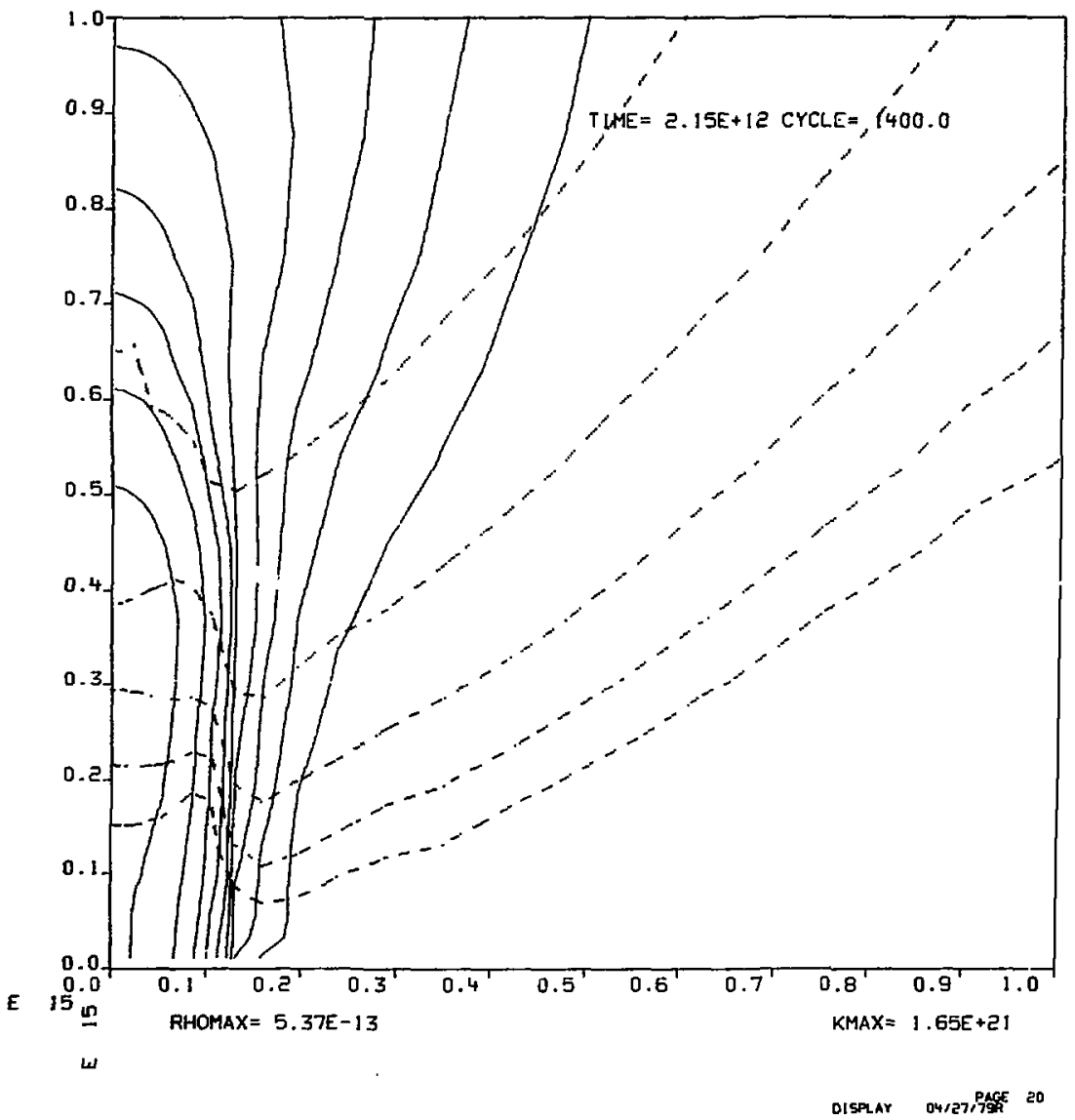

Frame $6 \mathrm{C} .2$ 


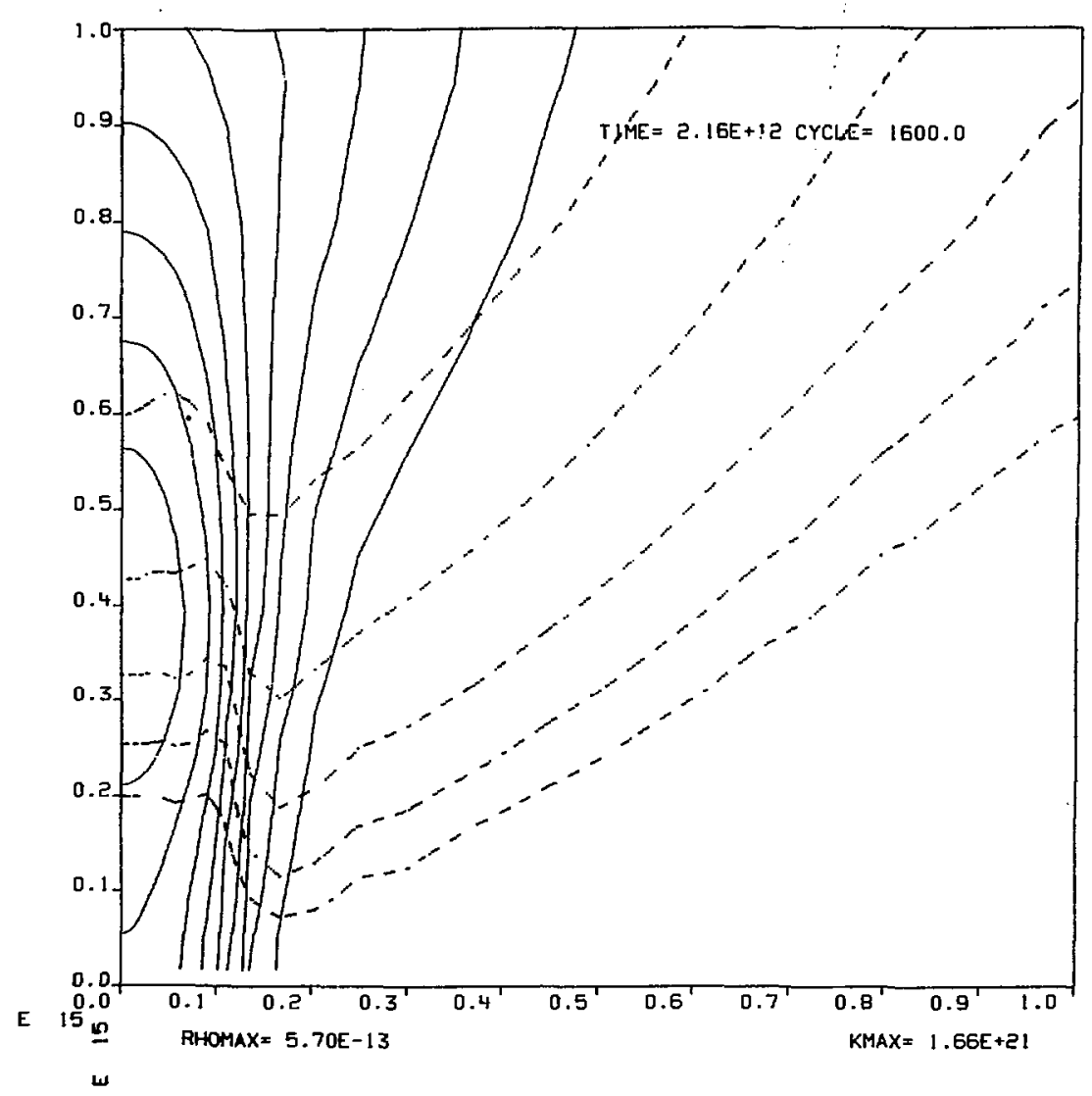

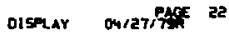

Frame 6C.3 


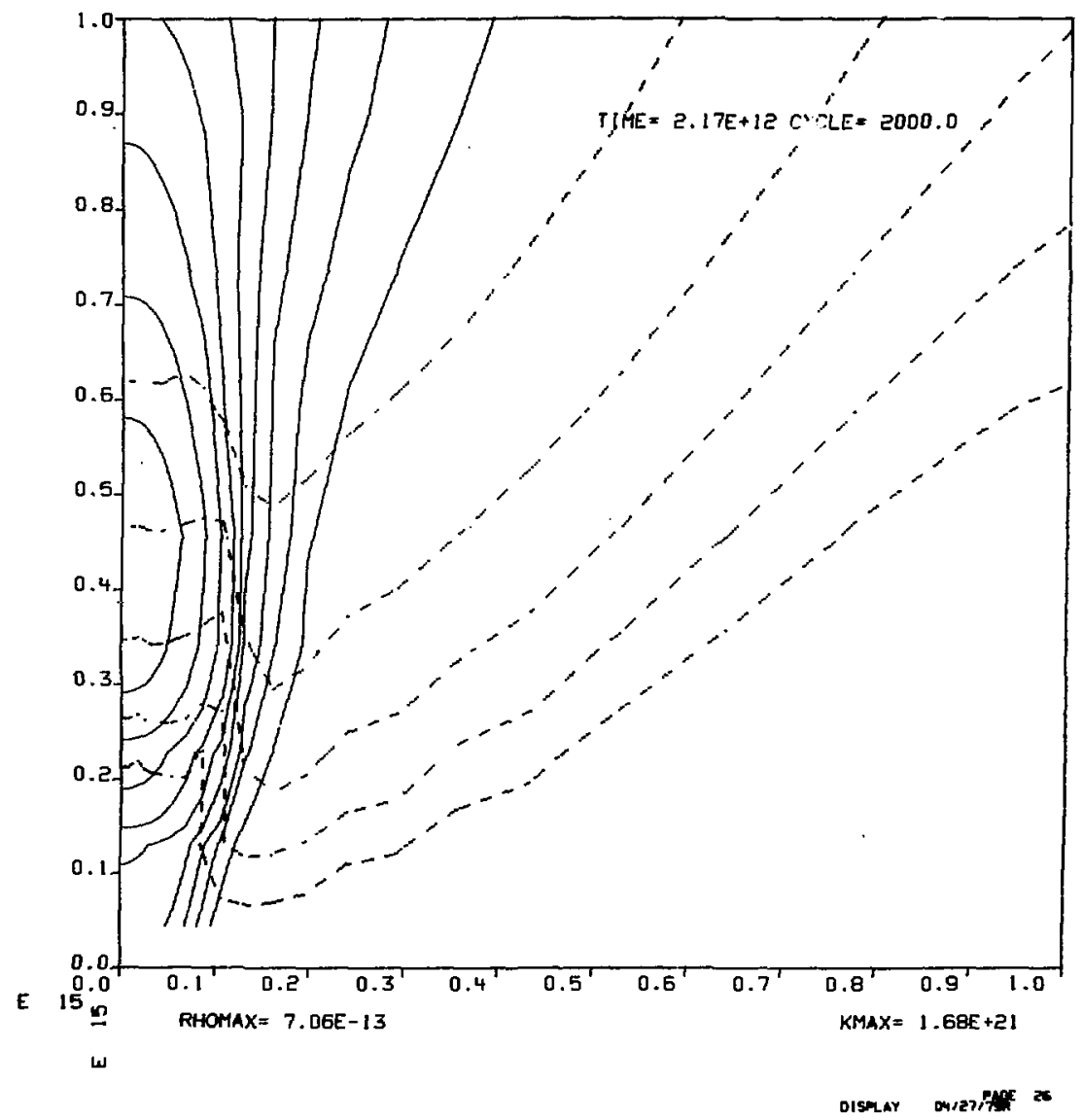

Frame 6 C. 4 
CHAPTER 7

DISCUSSION

We now discuss the relevance of the results presented in chapters 4-6 to ring formation in particular, and star formation in general. We showed in chapter 4 that the distribution of angular momentum determines whether equilibria (rotating Bonner-Ebert spheres) are disk or torus shaped. Specifically, for the distribution parameter $s=0.1$ a $r$ ing solution was round. and for $s \neq 1$ spheroidal solutions were round. Although only one ring equilibrium was calculated. the idea that collapse of an unstable cloud with $\mathbf{s}<$ 1 would produce a ring suggested itself. A verification calculation was described in chapter 5, case $5 \mathrm{~F}$. Collapse of an $s=0.1$ cloud leads to a centrifugal rebound which generates an off-axis density perturbation that grows to the point of gravitationally binding itself. The ring formation mechanism described by Tohline (1980) appears to be operative in evolution $5 F$. However isothermal collapse of $s \geqslant 1$ clouds do not form rings to the extent they have been calulated and described in chapter 5. Rather, we find a runaway collapse yjelding very high central concentrations and distributions of density and angular velocity suggestive of evolution to a singular state.

In collapse it is the approach to equilibrium, or at least force balance. that drives ring formation (see. fig. 5.10d). In the following simplified model of rotating isothermal collapse we study the structure and stability of singular disks that are consistent equilibrium final states for evolutions 
5A-5D. Structure and stability of disks with other distributions of angular momentum are considered as well.

7.1) A Simple Model of Rotating. Isothermal Collapse

In the absence of pressure, a self-gravitating, rotating cloud collapses to an infinitely thin disk in roughly one freefall time. The disk is unstable to axisymmetric and nonaxisymmetric modes of all wavelenglhs ind would quickly break up (Toomre 1964). Pressure retards the collapse and makes a disk-envelope structure. The disk has variable and finite thickness, and some or perhaps all on the modes of instability are stabilized. In this context we view ring formation as an off-axis Jeans instability that occurs in a disk with an angular momentum structure insufficient to stabilize certain long wavelength modes.

We shall now calculate the structure of a thin disk in equilibrium for certain distributions of angular momentum. We connect these slates to initial conditions (unstable clouds) possessing varying distributions of angular momentum by applying the conservation of angular momentum for each mass element. The disk structure is calculated assuming zero temperature. The local stability of these solutions to axisymmetric modes is determined as a function of position and cloud temperature. The assumption is that pressure gradients do not effect the structure of the disk. The effect of pressure on disk structure is subsequent ly considered. 


\section{P.1.1) Structure}

Consider a cylindrical cloud of uniform density $\rho_{i}$, radius $R_{i}$, and height h, with a power-law distribution of specific angular momentum

$$
K=n R^{2}=K: \stackrel{R}{(-)^{n}}
$$

where $R$ is $:$. A ndrical radius, and $n>-2$ lo prevent divergent angular momentum at the origin. Wc have chosen a power-law distribution of angular velocity rather than a linear one as used previously because the former admits a simple disk solution.

Assume collapse produces an infinilely thin disk of radius $R_{d}$, with surface density and specific angular momentum distributions

$$
\begin{aligned}
& 0=a_{d}\left(\frac{R}{R_{d}}\right)^{a}, 0 \geq a>-2 \text {. } \\
& K=k_{d} \underset{R_{d}}{R}(-, c),-(2+a), R \leqslant R_{d} \text {. }
\end{aligned}
$$

Wc want to find under what conditions equations (2) are consistent with hydrostatics, and then relate $\sigma_{d}, k_{d}, k_{d}, a$, and $c$ to $\rho_{i}, k_{i}, R_{i}, h$, and $n$.

$$
\begin{aligned}
& \text { Hydrostatics states } \\
& 0=\frac{\mathrm{K}^{2}}{\mathrm{R}^{3}}-g_{\mathrm{R}} .
\end{aligned}
$$

The gravitational acceleration, $g_{R}$, can calculated by integrating over hoops to yield

$$
g_{R}(R)=-2 \pi G C_{a}(R) o(R) .
$$

where 


$$
C_{a}(R)=\sum_{l=0}^{\infty} P_{2 l}^{2}(0)\left\{\frac{2 l+1}{2 l+2+a}-\frac{2 l}{2 l-1-a}\left[1-\left(-\frac{R}{R_{d}}\right)^{2 l-1-a}\right]\right\} .
$$

and $P_{\ell}$ are the Legendre polynomials. The coefficient $C_{a}$ is a weak function of $R$ for steep disks $(a<-1 / 2)$, and is approximately equal to $1 /(2+a)$ añay $i$ in the disk edge. From equation (3) it is easy to see that $K$ will be power-law only where $g_{R}$ is power-law. This is true away from the disk edge. Therefore we modify equation (2) to the following:

$$
\begin{aligned}
& 0=a_{0}\left(\frac{R}{R_{0}}\right. \\
& K=K_{0} \underset{R_{0}}{(-)^{c}}, R \leqslant R_{0} \leqslant R_{d} \text {, }
\end{aligned}
$$

where now $o_{0}$ and $K_{0}$ are constants at some reference radius $R_{0}$ vihere the power-laws are still a good approximation.

Substituting equations (2) and (4) into equation (3), and using the approximate form for equation (5), we find

$$
c=\frac{a+3}{2}
$$

and

$$
\frac{\mathrm{K}_{0}^{2}}{R_{0}^{3}}=\frac{2 \pi \mathrm{Ga} 0}{2+a}
$$

Therefore, the hydrostatic solution is

$$
\begin{aligned}
& \sigma=\sigma_{0}\left(\frac{R}{R_{0}}\right) \\
& K=\left(\frac{2 \pi G \sigma_{0}}{2+a} R_{0}^{3}\right)^{1 / 2}\left(\frac{R}{R_{0}}\right)(a+3) / 2
\end{aligned}
$$


under the same restrictions as given in equation (6). We shall now relate this solution to the initial cloud.

Equations (9) describe how angular momentum is distributed in the disk as a function of mass coordinate. Define a specific angular momentum spectrum

$$
\mu(k)=\int_{0}^{K} d u(k)
$$

lsothermal collapse conserves vorlicity lexcept near shock fronts, which appear to have a negligible effect on circulation). Therefore each mass element preserves its specific angular momentum. From equations (1.9) we have

$$
\mu(K)=\pi h \rho_{i} R_{i}^{2}\left(\frac{K}{K_{i}}\right)^{2 / n}=\frac{2 \pi \sigma_{0} R_{0}^{2} K}{2+a}(-)^{2(2+a) /(3+\theta)} .
$$

L.et $p$ be the exponent of $K$ in the angular momentum spectrum. One of $\sigma_{0}, K_{0}$, and $R_{0}$ is arbitrary. Choosing $K_{0}=K_{i}$, from equation:s ( 8.11 ) we find

$$
\begin{aligned}
& \mathrm{p}=\frac{2}{\mathrm{n}}=\frac{2(2+\mathrm{a})}{(3+\mathrm{a})}, \\
& \mathrm{R}_{0}=\frac{\mathrm{K}_{\mathrm{i}}^{2}}{\pi \mathrm{G} \rho_{\mathrm{i}} \mathrm{hR} \mathrm{R}_{\mathrm{i}}^{2}}, \\
& 0_{0}=\frac{2+\mathrm{a} \mathrm{R}_{\mathrm{i}}}{2}(-)^{2} \rho_{i} \mathrm{~h} .
\end{aligned}
$$

Equations $(13,14)$ simplify for an $n=2$ (uniformly rotating) initial state. Defining $\Omega_{i}=K_{i} / R_{i}^{2}$ and $\Omega_{0}=K_{0} / R_{0}^{2}$, setting $R_{1}=R_{0}$ in equations $(13,14)$, we have

$$
\begin{aligned}
& R_{0}=\frac{\pi \rho_{i} h}{\Omega_{i}^{2}} . \\
& o_{0}=\frac{\rho_{i} h}{2} .
\end{aligned}
$$


Thus, given an initial cloud specified by $\rho_{i}, K_{i}, R_{1}$, and $n$, we have an approximate solution for the final disk state. In particular, disks with power-law distributions of surface density and angular velocity are predicted to arise from clouds with power-law distributions of angular momentum as def ined by equation $(10)$

To show this model has validity, figure 7.1 shows a comparison of the computed (solid line) and predicted distributions (dotted line) for evolution 5B. Case 5B concerned the collapse of a uniform, rigidly rotating cloud. Such a cloud has $\mu(k) \sim K$, therefore $\quad \mathrm{a}=-1$. The $0 \sim R^{-1}$ and $K \sim R$ features are fairly well represented. The offset is due in part to a neglect of pressure gradients in the model calculation.

\subsection{2) Stability}

We now consider the stability of the singular thin disk solution given by equations (9). Toomre (1964) has derived a local stability criterion for a rotating thin disk of stars to axisymmetric modes by a linear analysis. Repeating his calculation for a thin disk of gas yields the following critical local soundspeed, acrit, for stability:

$$
a_{\operatorname{cjt}}=\frac{\pi \mathrm{G} \sigma}{\kappa} \text {. }
$$

where 0 is the local surface density, and

$$
x=2 \cap\left(1+\frac{\operatorname{ld} \ln \Omega}{2 \mathrm{~d} \ln R}\right)^{1 / 2}
$$

the epicycle frequency. Substituting equations (9) into equation (17) we find 


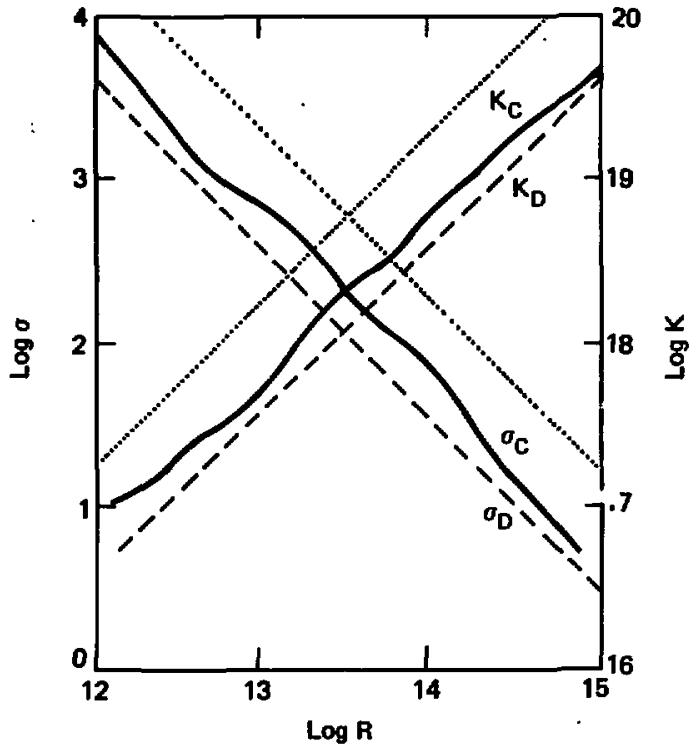

1

Figure 7.1--Surface density and specific angular momentum distributions as a function of radius in the equatorial plane of case $5 B$ (solid lines), and predicted distributions from thin disk analysis without pressure (dotted lines), and with pressure (dashed lines). 


$$
a_{c r i t}=\left(\frac{1}{a+3}\right)^{1 / 2} \frac{n G \sigma_{0} R_{0}^{2} R}{K_{0}} \underset{R_{0}}{(-)}(a+1) / 2 .
$$

Rewriting using the slope of the specitic angular momentum spectrum, p, we have

$$
a_{c r i l}=\left(\frac{2}{2-p}\right)^{1 / 2}-\frac{\pi G \sigma_{0} R_{0}^{2} R}{K_{0}}(-) R_{0}(p-1) /(2-p) .
$$

Now a curjous thing reveals itself. Let the cloud sound speed be a, and define a critical radius $R_{\text {crit }}$ such that $a_{c r j t}\left(R_{c r \mid t}\right)=a$. Then equation (lg) predicts ring formation for $R>R_{c r i t}$ for $p>1$, ring formation for $R<R_{c} i t$ for $p<1$, and for $p=1$ (Lhe distribution of a uniform, rigidly rotaling cloud), either ring formation or stability at all radi depending on a. In this sense, uniform rigidly rotating clouds are very special initial conditions. The situation is shown schematically in figure 7.2. An at tempt to make this result quantitative is not given here because, except for the $p=1$ cases (5A-5D), we have not calculated power-law clouds as they are physically artificial. However it is plausible that the qualitaljue features of this argument are preserved for other distributions of angular momentum that we cannot analyze so simply. Our numerical examples seem to bear this conjecture out. Calculations $5 F$ and $5 G$ start from steep and shallow angular momentum distributions, respectively. however only $5 F$ shows ring formation. He can understand this apparent discrepancy by noting that case $5 \mathrm{G}$ is far from equilibrium at the end of our calculation (see figure $5.6 d$ ).

The stability criterion for $p=1$ disks can be simply expressed in terms of the injtial cloud parameters by substituting equations $(15,16)$ into equation (19), thus 


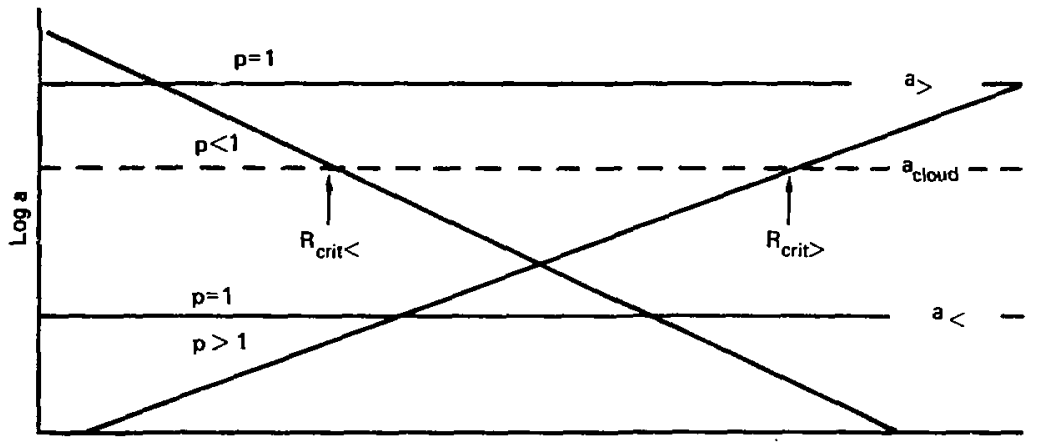

$\log \mathbf{R}$

Figure 7.2--Schematic of the radial dependence of the local soundspeed, $a$, needed to stabilize ring growth in thin disk solutions of differing angular momentum spectrum exponent, p. Isothermal disk of soundspeed $a_{c l o u d}$ is

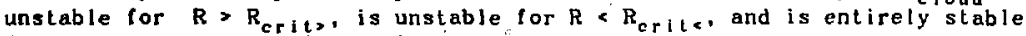
$\left(a=a_{c}\right)$, or unstable $\left(a=a_{>}\right)$. 


$$
a_{c+i l}=\frac{\pi G \rho_{i} h}{\sqrt{8} \Omega_{i}} .
$$

Substituting the parameters for cases 5A - 5D into equation (20), we find acrit $=4.7 a, 2.35 a, 1.67 a$, and $1.36 a$, respectively. Therefore by this simple analysis evolutions $5 \mathrm{~A}$ - $5 \mathrm{D}$ are predicled to eventually become $\mathrm{ring}$ unstable. We have ignored the effects of pressure on the disk structure in obtaining this result, however. We turn to these matters next.

7.1.3) Hydrostatics lncluding Pressure

Pressure modifies disk structure directly by adding a pressure gradient term to the radial hydrostatic equation, and indirectly through finite thickress effects on the gravitational force. Both. effects arc considered here in an approximatc way. Rather than solving for disk solutions with pressure, we inquire under what conditions equations (9) apply.

The equations of hydrostatics are

$$
\begin{aligned}
& 0=\frac{k^{2}}{\mathrm{k}^{3}}+g_{\mathrm{R}}-\frac{1 \partial \mathrm{P}}{\rho \partial \mathrm{R}} \\
& 0=g_{Z}-\frac{1 \partial \mathrm{P}}{\rho \partial \mathrm{Z}} .
\end{aligned}
$$

Here $g_{\mathrm{R}}$ and $g_{\mathrm{Z}}$ are the components of the gravitalional acceleration, and $\mathrm{P}=$ $\rho a^{2}$. Assuming the disk is thin, replace the $z$ component of the hydrostatic equation with the approximate formula

$$
\rho=\frac{\sigma}{h_{\text {eq }}},
$$

where 


$$
h_{e q}=\frac{a^{2}}{4 \pi G a}
$$

the equilibrium scale height for an isothermal disk.

The assumption that pressure is unimportant for the disk solution (9) is checked by evaluating the ratio of pressure to gravitational acceleration,

$$
x=\underset{\partial R}{\mid \frac{\partial P}{\partial R}}=\frac{\left|2 a+a^{2}\right| a^{2} R}{\pi G \sigma_{0} R_{0}}\left(R_{0}\right)^{-(1+a)} \sim R^{(2-2 p) /(2-p)} .
$$

If $x \ll 1$, then equations (9) are a good approximation to the solution. However we see from equation (24) that for $p \neq 1, x$ can becone large; some radius. The power-law solution (9) is not even approximately correct in this case. Only for $p=1$ will a single power-law describe the disk structure. Turning to this case then, we find equations (9) apply if $k_{0}$ satisfies

$$
\frac{\mathrm{K}_{0}^{2}}{\mathrm{R}_{0}^{3}}=\frac{2 \pi \mathrm{G} \sigma_{0}}{2+\mathrm{a}}+\frac{2 \mathrm{aa}^{2}}{\mathrm{R}_{0}}
$$

Finally, to check if the disk is indeed thin we form the ratio $h_{e q} / R$ for the new $p=1$ solution. We $f$ ind, using equations $(9,15,16,20,23,25)$.

$$
\left.\frac{h_{e q}}{R}<\frac{1 a}{16 a_{\mathrm{crjt}}}\right)^{2}
$$

The crilical soundspeed for stability, $a_{c}$ it. remains unchanged from equation (20) because $R_{0}$ drops out. Since $a<a_{\text {cit }}$ for cases 5A-5D, the conclusion that they will eventually form rings still stands.

A recalibration of $K_{0}, \sigma_{0}$, and $R_{0}$ for case $5 B$ yields the dashed solution in figure 7.1. It appears the cloud has overshot equilibrium slightly. It is passible that the subsequent eentrifugal rebound of this disk will excite a ring instability. This remains to be computed. 
Under certain restricting assumptions, ${ }^{1}$ the analysis predicts ring instrbility inside a critical radius for a shallow distribution of angular momentum, and ring instability beyond a critical radius for a steep distribution of angular momentum. Ring formation is the shallow case may not occur, however, if the critical radius lies inside the boundary of the nonisothermal core, which invariably forms before isothermal equilibrium is reached.

\section{2) Ring Controversy}

Ring formation in rotaling collapse became a point of controversy because some workers found rings and some did not, all proceeding from the same initial conditions (see review in chap. 1). In purely isothermal collapse the numerical reality is that either a ring forms or the cloud becomes so centrally condensed that it is a losing battle to resolve the central flow. In view of the fact that essentially every calculation in the ljterature started from uniform density, rigidly rotating spheres (Larson 1972: Tscharnuter 1975; Black and Bodenheimer 1976; Fricke, Mollenhof f, and Tscharnuter 1976; Nakazawa, Hayashi, and Takahara 1976; Kamiya 1977; Boss 1979; Norman, Wilson, and Barton 1980), and in view of she fact that we showed in the previous section that a singular disk is a consistent tinal state for such a cloud, and in view of the fact that evolutions 5A-5D appear to be evolving to singular states, or at least ones very difficult to resolve, in good agreement with the simple analysis (see figs. 5.8 , 7.1). we attribute the ring discrepancy to the following factors:

(I) numerical diffusion of angular momentum, either inward or outward, which effectively changes the central distribution of angular momentum

1 The analysis ignored tinite thickness effects. A f inite thickness stabilizes modes of wavelength short compared to the thickness (Toomre 1964). 
enough to carry the evolution onto either a ring or disk evolution. respectively

(2) Iailure to adequately resolve the highly convergent flow which leads to ring formation because of poor force representation.

That a diffusive treatment of angular momentum advection can change the specific angular momentum spectrum has been demonstrated by Norman, Wilson, and Barton (1980).

7.3) $\beta$ and Ring Formation

Maclaurin spheroids are secularly and dynamically unstable to an axisymmetric mode for $\beta=0.37$ and 0.44 , respectively (Bardeen 1971). For this reason, $\beta$ was naturally taken as an indicator of the likelyhood of $r$ ing formation in cloud collapse. Black and Bodenheimer (1976) were thus at a loss to explain ring formation with $\beta \sim 0.23$ in a series of collapse evolutions. From the results of chapter 4, we see that not only can a ring be excited from an isothermal equilibrium spheroid at $\beta \sim 0.40$, but that ring equilibria are naturally occurring at significantly lower $\beta(\beta<0.20 !)$. It is reasonable to expect that ring equilibria exist with a range of $\beta^{\prime} s$, depending on their angular momentum and their distribution function. The spheroids arising from relaxing a uniform, rigidly rotating sphere may behave more like Maclaurin spheroids than isothermal spheroids with more shallow distribution of angular momentum. The latter may have significantly different critical numbers, or may be stable altogether. A survey of equilibrium isothermal spheroids with a variety of distribution functions will be undertaken to explore these possibilities. 
$7.1)$ Star Formation

The overriding purpose of this research is the elucidation of the processes of star formation. The discovery of ring equilibria (chapter 4), and the confirmation of ring structures being produced in some collapses (chapter 5 ) give much support to the ring fragmentation scenario of Bodenheimer (1978). However, the process may not be hierarchical as he suggests. As we have scen. the distribution of angular momentum has a large effect on ring formation. 'There have been no $3 \mathrm{D}$ calculations with sufficient resolution to determine the angular momentum distribution in a ring fragment. The evolution of the ring fragments may be quite different from the parent cloud that produced them.

The discovery of ring growth accompanying core formation (chapter 6) provides another avenue for eloud Iragmentation operative at smaller scales and higher densities. In nature, a bar mode instability would probably occur before the ring instability set in because of its lower $\beta$ threshold. Indecd, it may be that core fission occurs at the moment of core formation bccause only then is there sufficient energy to completely disrupt the ccre.

We do not know the fate of those evolutions that did not form rings. Obviously, if the isothermal assumption is retained and the cloud is tending to a singular disk, no amount of resolution will be sufficient. However, nature becomes nonisothermal after a point. We have only calculated a few core oscillation periods into that part of the evolution. To continue would require prohibitively long integration times and the proper physics (radiation tlow and a gas-dust equation of state). Currently, a combined explicit/implicit hydrodynamic method is being developed in collaboration with Woodward (LLL), and Winkler (MPI) to attack the very difficult accretion phase. 
It is at ractive to identify clouds with shallow distributions of angular momentum as the progenitors of planetary systems. If the angular momentum of the core is small enough at formation, it will settle into a spheroidal configuration. As it accretes mass and angular momentum, it will contract and heat. When hydrogen dissociates a second collapse phase will ensue. A second core will form when dissociation and jonization are complete. This core may fission or fragment during lormation, or may remain a stable accretion centcr if its angular momentum in small enough and it has some ray of shedding excess angular momentum. The core may shed angular momentum hydrodynamically when it becomes bar unstable. Larson (1980) has suggested that hydrodynamic core readjustment may be responsible for the FU Orionis phenomena. Clearly much hydrodynamic simulation is needed to test these ideas. 


\section{CHAPTER 8}

CONCLUSIONS and FURTHER STUDY

An understanding of the structure and stability of interstellar gas clouds is important for star formation theory. Under the assumptions of isothermality and axisymmetry, a rotating gas cloud in pressure equilibrium with the intercloud medium is spheroidal or toroidal in shape depending on its internal distribulion of angular momentum per unit mass, and the relative amounts of its internal, rotationul, and gravitational energy. In particular, for a cloud with appreciable amounts of each kind of energy, and using the distribution of angular momentum of a uniform, rigidly rotating sphere as a reference. toroidal clouds have steeper distributions and spheroidal clcuds have more shallow distributions.

Rotation can increase the critical mass for gravitational instability by an order of magnitude over the crilical Bonner-Ebert sphere. An unstable cloud having an initially small amount of rotational energy compared to gravitational energy may be spheroidal, even though it has a steep distribution of angular momentum. As collapse proceeds, however, a ring stricture develops when the energies become comparable. An unstable cloud having a more shallow distribution of angular momentum and small total angular momentum may collapse to very high central densities and form a core before ring formation begins. When the center of the cloud becomes opaque to radiation, a flattened core forms whose structure depends on its amount and distribution of angular momentum. The classical instabilities of rotating, equilibrium spheroids may then grow. 
Limited to axisymmetry, the core evolves to a ring when its integrated ratio of rotational to gravitational energy, $\beta$, exceeds roughly 0.4 . The evolution of cores that form with much smaller $\beta$ may be quite different, however. Convection in the hydrogen dissociation zone may transport outwards significant amounts of angular momentum. This evolution is beyond the scope of the present work.

Rings are expected to exist in nature on the basis of this work: as stable interstellar clouds, and as transients in both the isothermal and nonisothermal phases of cloud collapse. Ring fragmentation presents itself as a workable mode of cloud fragmentation.. and perhaps a partial solution to the "angular momentum problem".

Much work remains to be done. More $2 D$ calculations of ring equilibria are needed to establish their properties. Equilibrium rings would serve as well defined initial conditions for $3 D$ studies of their stability to nonaxisymmetric modes. One of the most important but most difficult goals of such a study is the amount and distribution of angular momentum in the rragments. The properties of rings forming in collapse must also be tabulated for a variety of initial conditions. A predictive theory of cloud fragmentation by this mechanism would come out of putting this information together. The calculation of the evolution of a callapsing cloud through core formation and the subsequent accretion phase will require a code of great zoning flexibility, the proper microphysics, and coupled implicit-explicit hydrodynamics. 3D studies of core stability should also be done. 
APPENDIX A

TEST PROBLEM:RAPIDLY ROTATING POLYTROPES

The ability to produce an accurate rotating equilibrium hydrodynamically is tested by calculating the cooling sequence for a rotating $n=1.5(\gamma=5 / 3)$ polytrope. The distribution of angular momentum is that of a uniform, rigidly rolating sphere. The results of this test can be applied directly to sequence $\left(n=1.5, n^{\prime}=0.\right)$ of Bodenheimer and Ostriker (1973). The procedure is identical to that described in chapter 4, only here the entropy of the cloud is slowly reduced and not the temperature. The pressure outside the spheroid was sel to $10^{-4}$ the central pressure as a numerical necessity. Even so, the pressure virial amounted to less than a percent of the total energy of the system.

Figure Al shows a meridional section of the equipotential surfaces for several models along the sequence. Models of high $\beta$ and degree of flatness can be produced with no extra effort, unlike the self-consistent-ficld method of Ostriker and Mark (1968). Table AI shows a comparison of 2 models generaled with the self-consistent-field (SCF) method with those generated hydrodynamically (HYDRO). Call these models A and B. Our numbers have been normalized to the set of units based on $\left(G, M, \xi_{e}\right)=(1,1,1)$. The agreement is fajrly good, except cor ncenter and penter. This may be because these quantities are normalized by $\xi_{e}^{3}$ and $\xi_{e}^{3 / 2}$. respectively, and $\xi_{e}$ is poorly determined with such a crude interface method. To check this, we form the ratio $\eta_{c e n t e r}^{2} / \rho_{c e n t e r}$. which eliminates the normalization on $\xi_{e}$. This gives the 
bottom row of numbers. The agreement is good to a tew percent on this basis. We conclude that the normalization is responsible for the discrepancies between our $D$ and $\rho$ and the published numbers.

A better system of units for nondimensionalizing the results are based on $(G, M, a)$. where $a$ is the central soundspeed. $J, \Omega$, and $\rho$ are given in these units in table A1. shown in parenthesis.

TABLE Al

\section{A COMPARISON OF RAPIDLY ROTATING POLYTROPE SOLUTIONS: $\left(n=1.5, n^{\prime}=0.\right)$}

MODEL A

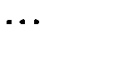

$\beta$

$J \cdot$

Denter

Peenler *

$\xi_{e} / \xi_{p}$

$\Omega^{2} / \rho$
$\mathrm{SCF}$

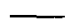

$$
0.137
$$

0.234

1.60

2.49

1.89

1.03
HYDRO

0.139

1.95
MODEL B

SCF

HYDRO

0.263

0.256

0.340

$0.348(0.255)^{\cdots}$

$0.243(0.205)^{\circ *}$

2.53

$2.26(5.70)^{\cdots}$

$1.45(2.42)^{\circ}$

5.24

$4.19(26.76)^{\star \bullet}$

$1.95(5.44)^{* \bullet}$

3.78

3.94

1.22

1.21

- $\left(G, \mu, \xi_{e}\right)=(1,1,1)$

$\because(G, \mu, a)=(1,1,1)$ 

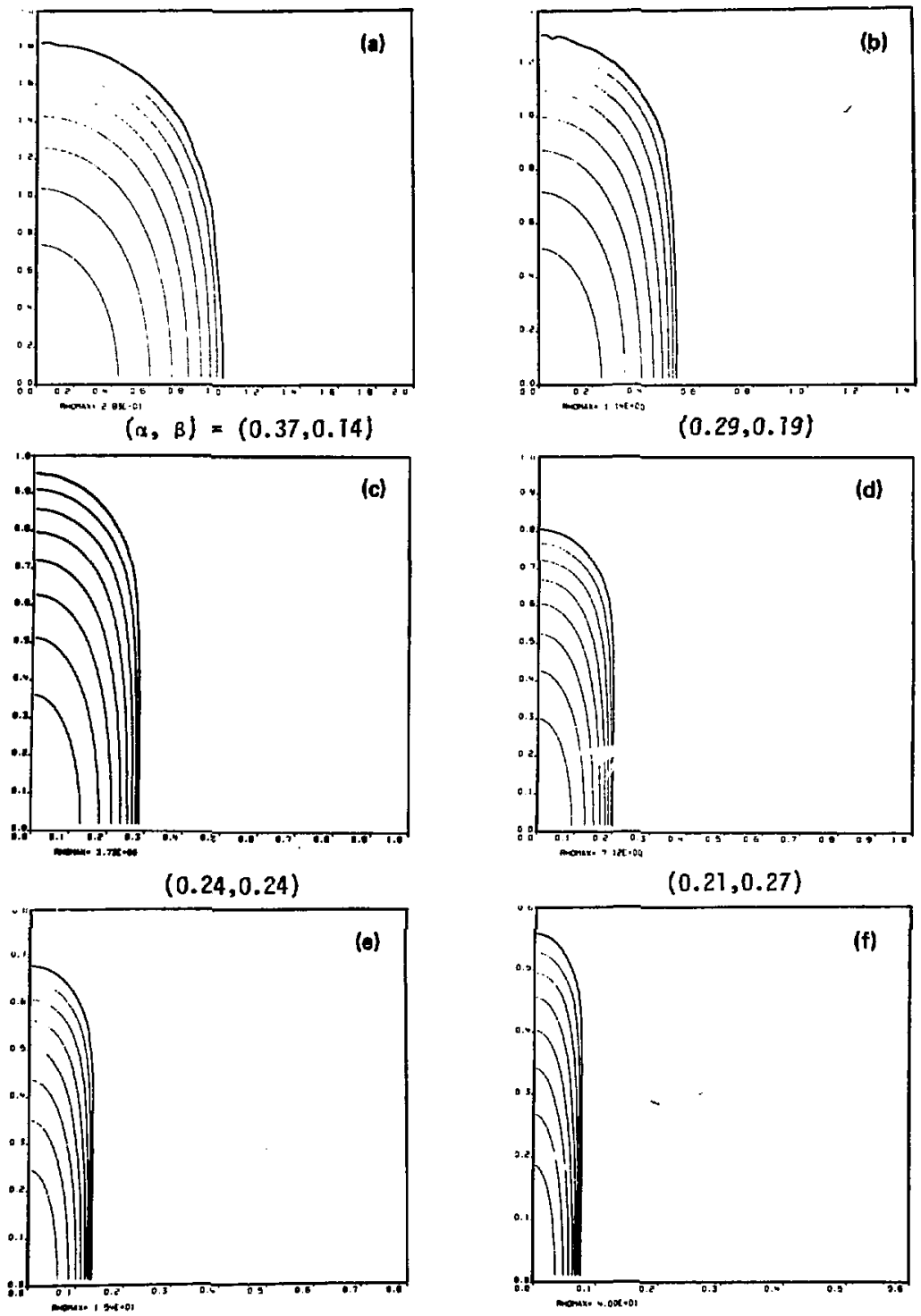

$(0.18,0.31)$

Figure Al

$(0.13,0.36)$ 
Figure Al-Density isocontours in meridional plane of rapidly rotating polytropes $\left(n=1,5, n^{\prime}=0\right)$ of increasing $\beta$. Energy ratios $\alpha$ and $\beta$ are shown in parenthesis. Virial theorem states $\alpha+\beta=1 / 2$. 


\section{APPENDIX B}

DENSITY AND ANGULAR VELOCITY IN THE CENTER OF A COLLAPSING CLOUD

In chapter 5 we showed evidence ( $f$ igure 5.7 ) that $n \sim \rho^{1 / 2}$ in the center of a collapsing. rotating. isothermal cloud. Here we present a simple derivation of this result.

Consider a uniform cylinder of radius $r$, height $h$, density $\rho$, and angular velocity 0 . If we idealize the collapse of the cloud as the collpase of such a cylinder so that the unllorm nature of $\rho$ and $\theta$ are preserved, then we can predict how $h$ and $n$ change during collapse provided one further assumption is made: the height $h$ is assumed to always be the instantaneous equilibrium scale height for an isothermal disk:

$$
h=h_{e q}=\frac{a^{2}}{4 \pi \mathrm{G} 0}=\left(\frac{a^{2}}{4 \pi \mathrm{G} \rho}\right)^{1 / 2} .
$$

where the latler expression comes from assuming $\sigma=\rho h$. This assumption is a good one whey the cloud becomes quite flattened. Equation (1), plus the equations for the conservation of mass and angular momentum in the cylinder yield the following system of equations upon logarithmic differentiation:

$$
\begin{aligned}
& 2 \mathrm{~d} \ell_{\pi r}+\mathrm{d} \ell_{\pi h}+\mathrm{d} \ell_{\pi \rho}=0 . \\
& \mathrm{d} l_{\pi \Omega}+2 \mathrm{~d} \ell_{\pi r}=0 . \\
& 2 \mathrm{~d} \ell_{\pi h}+\mathrm{d} l_{\pi \rho}=0 .
\end{aligned}
$$

The first and third equations give $\mathrm{d} \ln r / \mathrm{d} \ell n h=1 / 2$, or $r^{2} / h=$ constant. Using this relation to eliminate $r$ altogether in the second equation we produce the desired result: 
$\frac{\mathrm{d} \ln \Omega}{\mathrm{d} \ln \rho}=\frac{1}{2}, \quad$ or $\Omega \sim \rho^{1 / 2}$.

This result is mare general than it seems. Suppose we have a force field 2 that leads to a flattened isothermal system. If $2 r^{2}$ is conserved in a cylindrical element as collapse proceeds, then the above considerations tell us that $2 \sim \rho^{1 / 2}$ at the center of the cloud. Scolt and 8 lack (1978) have noted $B \sim$ $\rho^{1 / 2}$ in the cesier of a collapsing. nonrotating. magnetized cloud, where $B$ is the magnetic flux density. The calculation assumes flux-freezing, which insures that $B r^{2}$ is conserved. 


\section{REFERENCES}

Bardeen, J. M. 1971, Ap, J., 167, 425 .

Black, D. C., and Bodenheimer, P. 1975, Ap. J., !99,619. . 1976, Ap. J., 206, 138 .

Bodenheii er, P. 1978, Ap. J., 224, 488 .

Bodenheimer, P., and Black, D. C. 1978, in Protostars and Planets,

ed. T. Gehrels (Tueson: University of Arizona Press), p. 288 .

Bodenheimer, P.. and Ostriker, J. P. 1973, Ap. J., 180, 159.

Bodenheimer, P., and Tscharnuter, W. 1979, Astron. Ap., in press.

Bonner, W. B. 1956, M.N.R.A.S., I16, 351 .

Boss, A. P. 1979, Ph.D. thesis, University o: California, Santa Barbara

deBar. 12. B. 1974, Fundomentals of the KRAKEN Corie.

Lawrence Livermore Laboratory Internal Report 760.

Ebert, R. 1955, Zs. f.Ap., 37, 217 .

Fricke, K. J., Mollenhof, C., and Tscharnuter, W., 1976. Astron. Ap., 47, 407 .

Gaustad, J. E., 1963, Ap. J., 138, 1050 .

Kamjya, Y. 1977, Prog. Theor. Phys., 모, 802.

Larson, R. B. 1972, M.N.R.A.S., 1 $156,437$.

S.eblanc. S. M. private communications.

Nakazawa, K., Haysshi, C., and Takahąa, M. 1976,

Prog. Theor. Phys., 56, 515.

Norman, M. L., and Wilson, J. R, 1978, Ap. J., 224, 497.

Norman, M. L., Wilson, J. R., and Barton, R. T. 1980, accepted Astrophysical journal.

Ostriker, J. P., and Bodenheimer, P. 1973, Ap, J., 180, 171. 
Paccman, D. W., and Rachford, H. H. 3955 ,

J. Soc. Indust. Appl. Salh., 3,28 .

Scott, E. H., and Black, D. C. 1978, preprint.

Shu, F. H. 1977, Ap. J., 214, 488,

... Spit\%cr, l., 1978, in Physical Processes in the Intersieltar Ifedium (New York: Wiley), pl.

Tohline, J. F., 1980, Ap. J., 236, 1800.

Tassoul, J.-l. 1978, Theory of Rolating Slars

(Princelon: Princelon University Press), chap. 4.

Toomre, A, 1964, Ap, J., 139, 1217.

Tscharnuter. W. 1975, Astron. Ap., 39, 207.

c...... 1978, in The thoon and Planets, Vol. 19, no. 2.

Winkler, K.-II, and Newmen, M. I. 1980, Ap. J., 236, 201 .

Woodward, P. R. 1978, Ann, Rev. Astron. Astrophys., 16, 555. 